Florida International University FIU Digital Commons

4-2-2015

\title{
Hybrid Power System Intelligent Operation and Protection Involving Plug-in Electric Vehicles
}

Tan Ma

3011389,matan8571@gmail.com

DOI: $10.25148 /$ etd.FI15032175

Follow this and additional works at: https://digitalcommons.fiu.edu/etd

Part of the Automotive Engineering Commons, Controls and Control Theory Commons, Electrical and Electronics Commons, Power and Energy Commons, and the Systems and Communications Commons

\section{Recommended Citation}

Ma, Tan, "Hybrid Power System Intelligent Operation and Protection Involving Plug-in Electric Vehicles" (2015). FIU Electronic Theses and Dissertations. 1760.

https://digitalcommons.fiu.edu/etd/1760 


\section{FLORIDA INTERNATIONAL UNIVERSITY}

Miami, Florida

\section{HYBRID POWER SYSTEM INTELLIGENT OPERATION AND PROTECTION INVOLVING PLUG-IN ELECTRIC VEHICLES}

A dissertation submitted in partial fulfillment of

the requirements for the degree of

DOCTOR OF PHILOSOPHY

in

ELECTRICAL ENGINEERING

by

Tan Ma

2015 
To: Dean Amir Mirmiran

College of Engineering and Computing

This dissertation, written by Tan Ma, and entitled Hybrid Power System Intelligent Operation and Protection Involving Plug-in Electric Vehicles, having been approved in respect to style and intellectual content, is referred to you for judgment.

We have read this dissertation and recommend that it be approved.

Hai Deng

Armando Barreto

Jean H. Andrian

Berrin Tansel

Walid Saad

Osama A. Mohammed, Major Professor

Date of Defense: April. 2, 2015

The dissertation of Tan Ma is approved.

$\begin{array}{r}\text { Dean Amir Mirmiran } \\ \text { College of Engineering and Computing } \\ \hline \begin{array}{r}\text { Dean Lakshmi N. Reddi } \\ \text { University Graduate School }\end{array}\end{array}$

Florida International University, 2015 
(C) Copyright 2015 by Tan Ma

All rights reserved. 


\section{DEDICATION}

I dedicate this dissertation to my dear wife, Lilin Guo, whose patience, sacrifice, support, and understanding made the completion of this work possible, and to my beloved family who were unflinching in their support and prayers even from thousand miles away. 


\section{ACKNOWLEDGMENTS}

This dissertation would not have been possible without the help, support, resolute dedication, and patience of my principal supervisor, Professor Osama Mohammed. His passion for success has inspired me to take my own passions seriously, not to mention his advice and unsurpassed knowledge of the various fields of electrical power systems. Professor Mohammed has always provided me an endless supply of ideas, guidance, suggestions, and useful discussions. I am indebted for his enthusiasm, advice, moral as well as financial support, and friendship. I am also grateful for the chance he gave me to work at the Energy Systems Research Laboratory. There I found all the first-class equipment I needed to experimentally verify my results, and I developed professionally in an environment where work and engineering ethics are highly respected.

I wish to also thank the members of my committee for their support, patience, and valuable discussions. In particular, Dr. Hai Deng, Dr. Armando Batteto, Dr. Jean H. Andrian, Dr Berrin Tansel, and Dr. Walid Saad.

I would like to acknowledge the research support provided from the Department of Electrical and Computer Engineering at Florida International University. I also acknowledge the doctoral evidence acquisition fellowship and dissertation year fellowship from the FIU graduate school during the last period of dissertation research.

Thanks are also due to all the graduate members and undergraduate student scholars at the Energy Systems Research Laboratory, whose discussions, contributions, and assistance helped me achieve my research goals. I am also grateful to the FIU community and department staffs, especially Pat Brammer and Oscar Silveira, who have given me great help and support. 


\section{ABSTRACT OF THE DISSERTATION \\ HYBRID POWER SYSTEM INTELLIGENT OPERATION AND PROTECTION \\ INVOLVING PLUG-IN ELECTRIC VEHICLES}

by

Tan Ma

Florida International University, 2015

Miami, Florida

Professor Osama A. Mohammed, Major Professor

Two key solutions to reduce the greenhouse gas emissions and increase the overall energy efficiency are to maximize the utilization of renewable energy resources (RERs) to generate energy for load consumption and to shift to low or zero emission plug-in electric vehicles (PEVs) for transportation. The present U.S. aging and overburdened power grid infrastructure is under a tremendous pressure to handle the issues involved in penetration of RERS and PEVs. The future power grid should be designed with for the effective utilization of distributed RERs and distributed generations to intelligently respond to varying customer demand including PEVs with high level of security, stability and reliability. This dissertation develops and verifies such a hybrid AC-DC power system. The system will operate in a distributed manner incorporating multiple components in both $\mathrm{AC}$ and DC styles and work in both grid-connected and islanding modes.

The verification was performed on a laboratory-based hybrid AC-DC power system testbed as hardware/software platform. In this system, RERs emulators together with their maximum power point tracking technology and power electronics converters were 
designed to test different energy harvesting algorithms. The Energy storage devices including lithium-ion batteries and ultra-capacitors were used to optimize the performance of the hybrid power system. A lithium-ion battery smart energy management system with thermal and state of charge self-balancing was proposed to protect the energy storage system. A grid connected DC PEVs parking garage emulator, with five lithium-ion batteries was also designed with the smart charging functions that can emulate the future vehicle-to-grid (V2G), vehicle-to-vehicle (V2V) and vehicle-tohouse (V2H) services. This includes grid voltage and frequency regulations, spinning reserves, micro grid islanding detection and energy resource support.

The results show successful integration of the developed techniques for control and energy management of future hybrid AC-DC power systems with high penetration of RERs and PEVs. 


\section{TABLE OF CONTENTS}

CHAPTERS

PAGE

1. Introduction Error! Bookmark not defined.

1.1. Background 1

1.2. Current State of Hybrid Power Systems ................................................................6

1.2.1. Hybrid power system with renewable energy sources...................................

1.2.2. Plug-in electric vehicles charging in hybrid power system .......................... 10

1.2.3. Lithium-ion battery in power system and plug-in electric vehicles................16

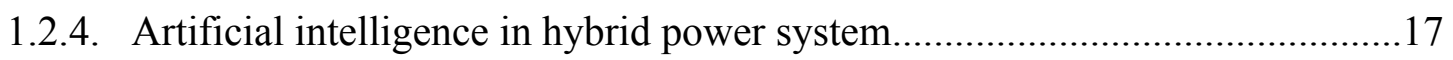

1.2.5. Information technology and wireless communication..................................20

1.3. Motivation for Solving the Existing Problems ..................................................21

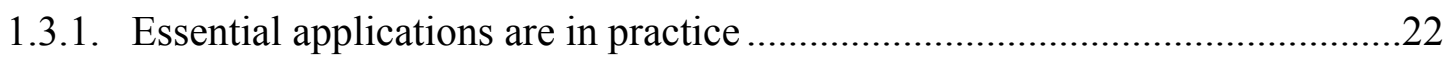

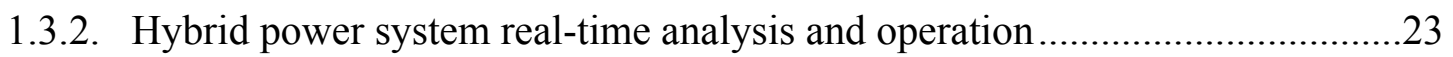

1.3.3. Smooth PEV charging impact to utility grid..............................................23

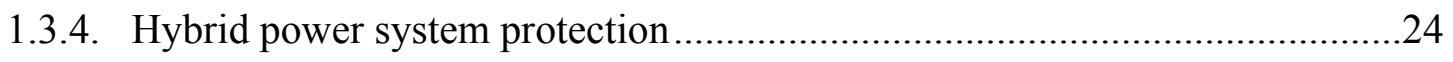

1.3.5. Verification of developed techniques on hardware based Testbed..................24

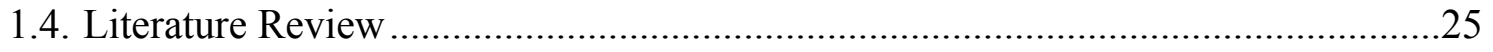

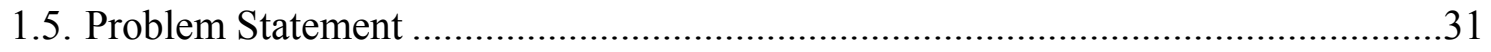

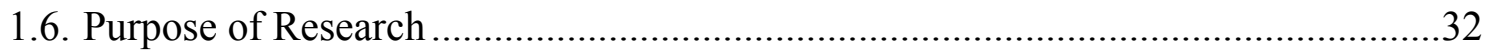

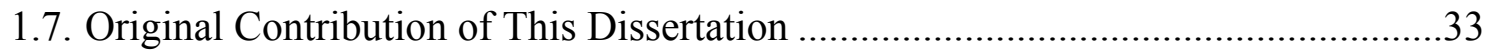

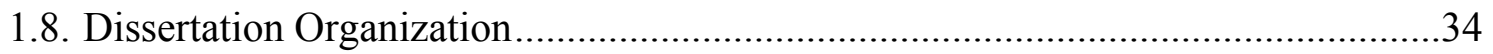

2. Hybrid AC/DC Power System with Plug-in Electric Vehicles..................................38



2.2. Hybrid AC-DC Power System Architectures...................................................42

2.2.1. Grid Connected Mode and Islanding Mode ................................................43

2.2.2. Hybrid AC-DC power system challenges................................................. 44

2.2.3. Hybrid AC/DC power system communication requirements ........................45

2.3. Plug-in Electric Vehicles Charging Impact to Utility Grid ..................................46

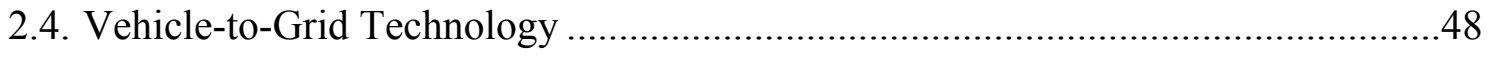

2.4.1. Vehicle to grid services.................................................................. 48 


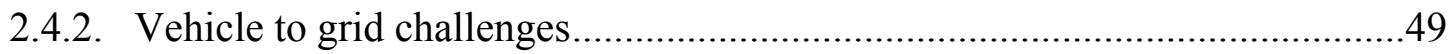

2.4.3. Vehicle to grid future market.......................................................................50

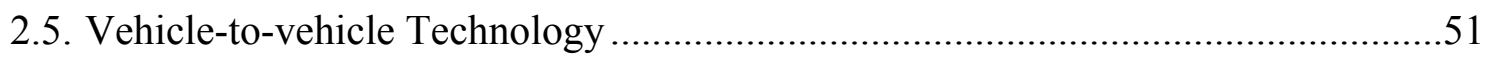

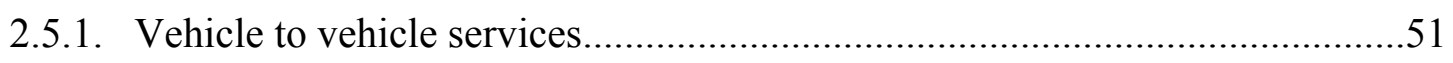

2.5.2. Vehicle to vehicle challenges....................................................................51

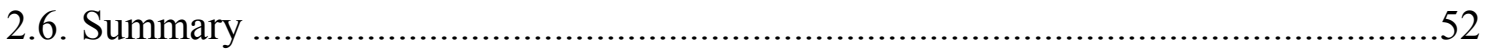

3. Integration of Renewable Energy Sources into Hybrid Power Systems.......................54

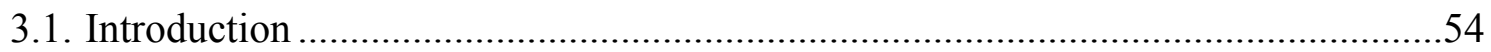

3.2. Renewable Energy Sources Characteristics Analysis .............................................56

3.2.1. South Florida solar energy source analysis...................................................56

3.2.2. South Florida wind energy source analysis ..................................................58

3.3. DC-DC Boost Converter with Maximum Power Point Tracking Ability

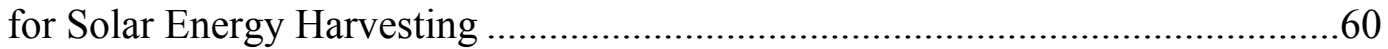

3.3.1. Photovoltaic panel MATLAB modeling.........................................................60

3.3.2. DC-DC boost converter MATLAB modeling ………………………...........62

3.3.3. Photovoltaic panel maximum power point tracking ........................................66

3.3.4. Photovoltaic panel emulator design ...............................................................69

3.3.5. DC-DC boost converter hardware design .......................................................

3.4. Power conversion topologies with wind turbines ..................................................71

3.5. Renewable energy sources impact to hybrid power systems ..................................74

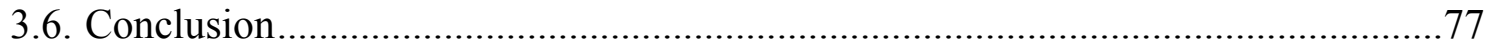

4. Optimal Renewable Energy Farm and Energy Storage Sizing Methodology for Future Hybrid Power System................................................................................79

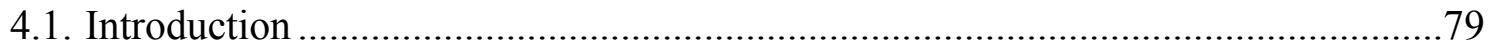

4.2. Modern Energy Storages Classification ............................................................... 81

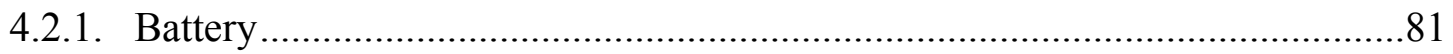

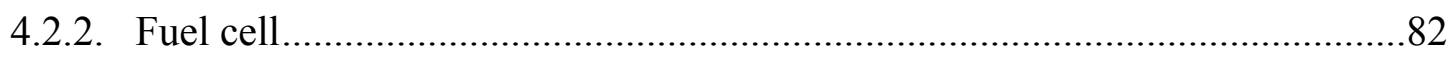

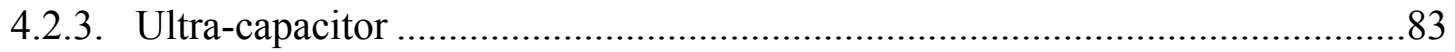



4.3. Local Load and Renewable Energy Sources Pattern Analysis..................................85 


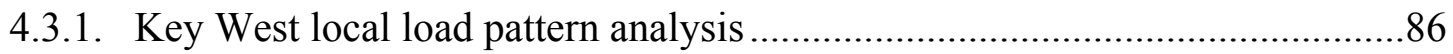

4.3.2. Key West renewable energy sources historical analysis................................86

4.4. Renewable Energy Farm Scale Optimization ...................................................91

4.4.1. Renewable energy farm scale optimization cost function design...................91

4.4.2. Optimization process by genetic algorithm ..............................................92

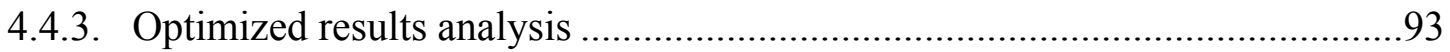

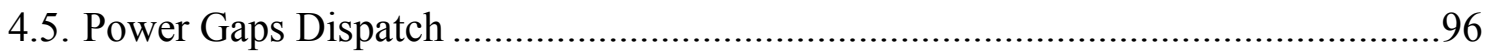

4.5.1. Hybrid power system stability issues with high penetration of renewable

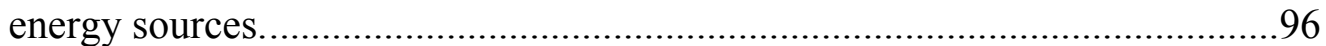

4.5.1. Impact limitation with energy storage systems ........................................97

4.6. Energy Storage System Scale Optimization.....................................................99

4.6.1. Lead acid battery charging/discharging mathematic model ..........................99

4.6.2. Ultra-capacitor model charging/discharging mathematic model..................101

4.6.3. Energy storage system scale optimization cost function design ...................102

4.6.4. Optimization process by genetic algorithm .............................................103

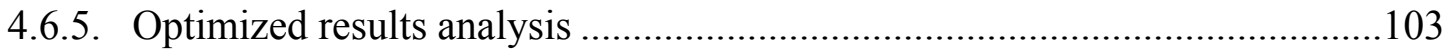

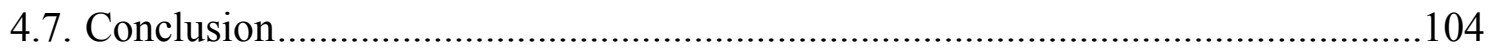

5. Development of High Accuracy Lithium-ion Battery Modeling with the

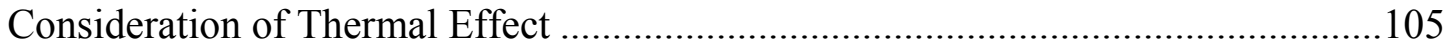

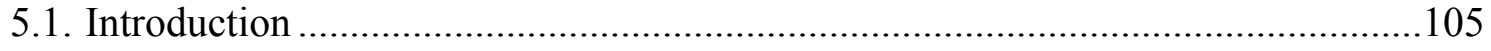

5.2. Advantages of Lithium-ion Batteries and Their Application................................ 107

5.3. Importance of Lithium-ion battery modeling and energy management systems

5.3.1. Over charge/discharge impact on lithium-ion batteries

5.3.2. Over heat impact to lithium-ion battery .................................................. 111

5.4. High Accuracy Lithium-ion Battery Model .................................................... 113

5.4.1. Lithium-ion battery SOC estimation...................................................... 115

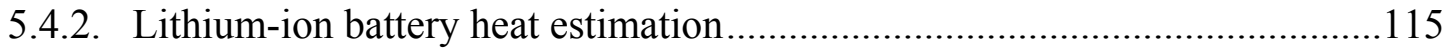

5.4.3. Real time lithium-ion battery SOC and thermal monitoring .......................116

5.5. Battery Bank Energy Management with SOC and Temperature Self-

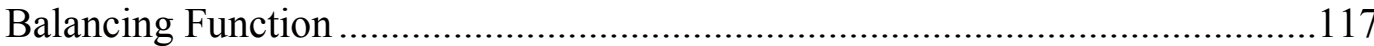

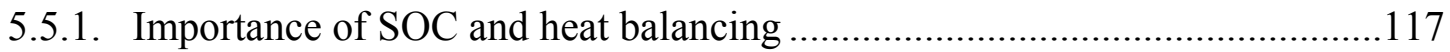


5.5.2. Fuzzy logic battery bank energy management algorithm 118

5.5.3. Battery bank SOC and temperature self-balancing circuit .122

5.5.4. Simulation and experimental verification .125

5.6. Real-time Battery module Operation in Hybrid Power Systems with

Droop Control.

5.6.1. Droop control in hybrid power systems.

130

5.6.2. Adaptive droop control for multi battery module operation in hybrid power systems.

5.7. Conclusion

6. Plug-in Hybrid Electric Vehicles Car Park Modeling and Design of Artificial Intelligent Power Management Algorithm ..........................................................136

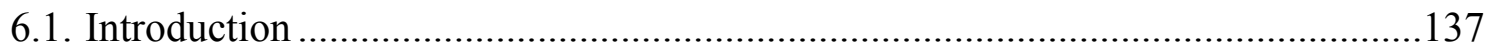

6.2. PHEV Car Park System Description and Problem Formulation ............................140

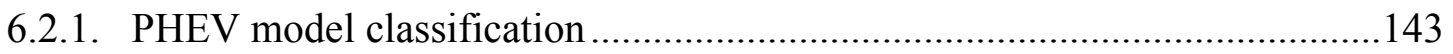

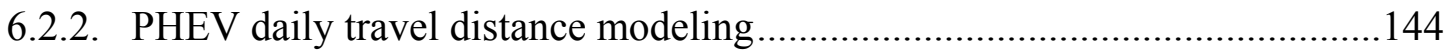

6.2.3. PHEVs parking duration modeling ...................................................... 145

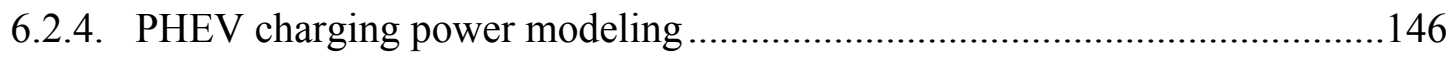

6.2.5. PHEV car park power requirement forecasting model ................................147

6.3. Fuzzy Logic Based Real Time Energy Management Algorithm ........................147

6.3.1. PHEV Charging Priority Levels classification ........................................ 148

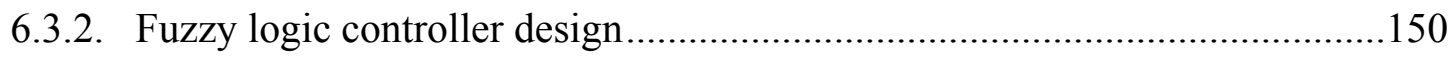

6.3.3. Maximizing the utilization of renewable energy sources ............................151

6.3.4. Minimizing the PHEVs' charging cost ................................................... 153

6.4. Fuzzy logic based PHEV smart charging case studies ......................................155

6.4.1. Small scale PHEV car park smart charging with maximum utilization of

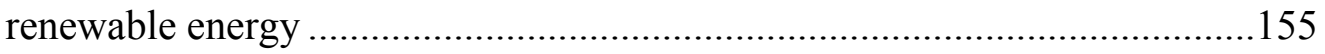

6.4.2. Small scale PHEV car park smart charging with consideration of local load and utility grid energy price .........................................................158

6.5. Fuzzy logic smart charging controller for a medium scale PHEV car park...........163



6.5.2. PHEV uncontrolled charging impact to the AC utility grid .........................165 
7. Economic Analysis of Real-time Large Scale PEV Network Power Flow Control Algorithm with the Consideration of V2G Services.................................................175

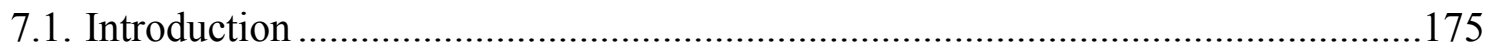

7.2. Large Scale PEV Network V2G secondary control potentials...............................178

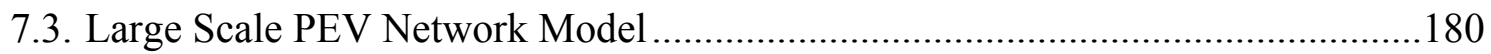

7.4. Analysis of Large Scale PEV Network Energy Consumption ..................................181

7.5. Analysis of Annual Local Load Pattern, Renewable Energy Resources

Pattern, and Grid Frequency Regulation Signal ..............................................185

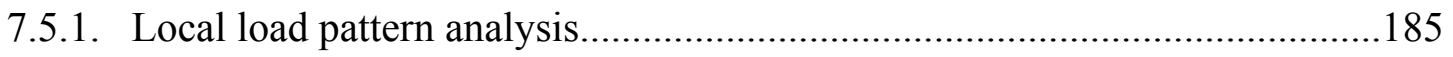

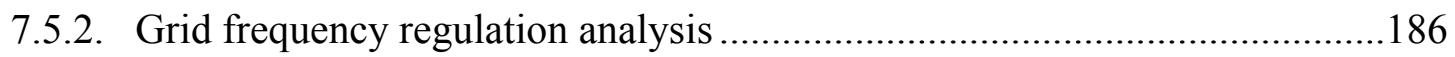

7.5.3. Renewable energy resources pattern analysis ................................................187

7.5.4. Optimal renewable energy farm design for the PEV network .......................187

7.6. Fuzzy Logic Based Real Time Energy Management Algorithm with

Consideration of Grid Frequency Regulation Services .....................................189

7.6.1. Fuzzy logic controller design................................................................190

7.6.2. Maximize the utilization of renewable energy sources....................................191

7.6.3. Regulating the grid frequency by using the PEV network ............................192

7.7. Overall System Economic Analysis ..................................................................192

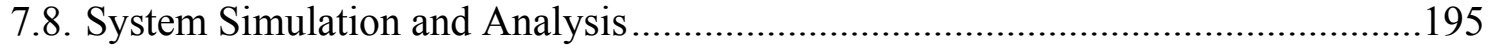

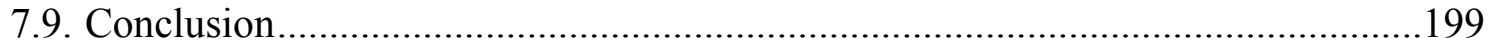

8. Bidirectional Energy Transfer and Power Electronics Devices Modeling,

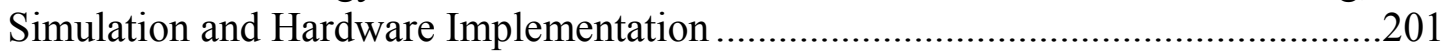

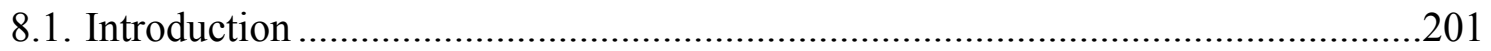

8.2. Hybrid AC-DC Power System Operation in Grid-connected Mode and

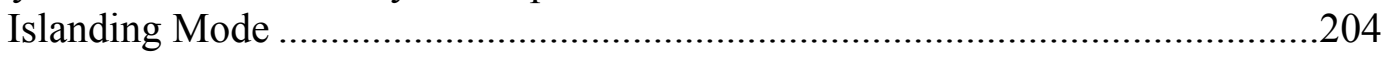

8.2.1. Hybrid AC-DC power system operation in grid-connected mode..................205

8.2.2. Hybrid AC-DC power system operation in islanding mode ............................205

8.2.3. Hybrid AC-DC power system islanding detection and frequency

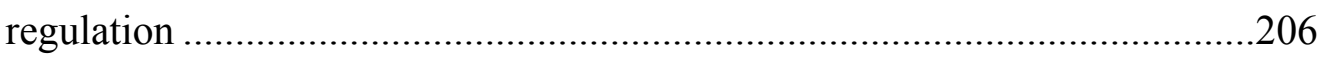

8.3. Bidirectional DC-DC Converter Control and DC Bus Voltage Regulation...........209

8.3.1. Bidirectional DC-DC converter topology …………………...........................209 
8.3.2. Bidirectional DC-DC converter current and voltage control..... .209

8.3.3. Bidirectional DC-DC converter hardware

8.3.1. Real time hardware experimental verification

8.4. Bidirectional AC-DC inverter control and AC Bus Frequency and Voltage

Regulation .215

8.4.1. Bidirectional AC-DC inverter topology .216

8.4.2. Bidirectional AC-DC inverter vector decoupling technique ..... 216

8.4.3. Bidirectional AC-DC inverter frequency and voltage control. 222

8.4.4. Bidirectional AC-DC inverter hardware .225

8.4.5. Real time experimental verification .226

8.5. Hybrid Power System Performance with Mitigation of Critical Loads 230

8.5.1. Maximizing power generation from photovoltaic panel emulator .230

8.5.2. Power flow in the hybrid AC-DC power system .232

8.5.3. Hybrid AC-DC power system simulation verification under pulse load .......236 8.6. Conclusion .244

9. Application of Microcontrollers and Wireless Transmitters for Monitoring and Control of Hybrid AC-DC Power Systems.

9.1. Introduction .245

9.2. Application of low-cost microcontrollers in a PEV car park emulator hybrid power system for power flow control and energy management. 248

9.2.1. dSpace controller for bidirectional AC-DC inverter control

9.2.2. STM32F4DISCOVERY controller for bidirectional DC-DC converter control

9.3. Application of wireless communication control in the PEV car park emulator hybrid power system.

9.4. Energy storage system operation with wireless communication and control

9.4.1. Battery banks SOC monitoring web server system design. .263

9.4.2. Bidirectional DC-DC converter power flow remote control .264

9.5. Conclusion .269

10. Laboratory Scaled Plug-in Electric Vehicles Car Park Infrastructure Emulator Design .270 


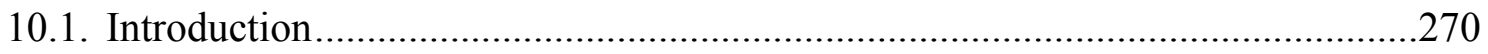

10.2. PEV car park infrastructure emulator overview ..............................................272

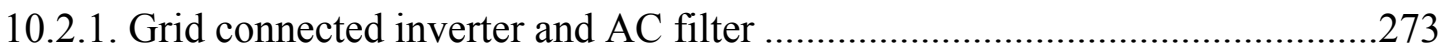

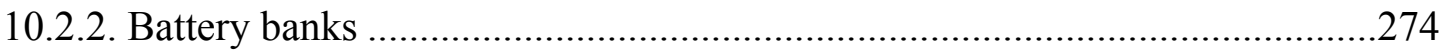

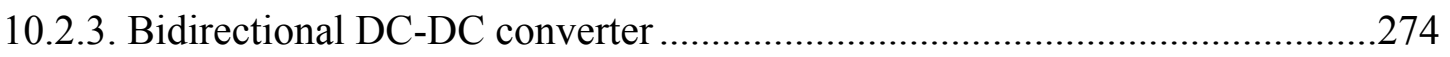

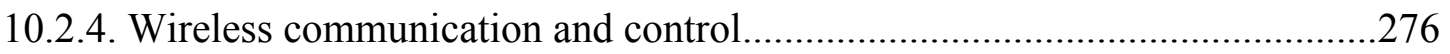

10.3. Application of a real time energy management algorithm to the car park



10.4. Hybrid AC-DC power system with PEV car park performance analysis ............279

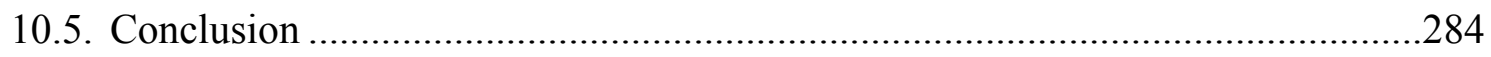



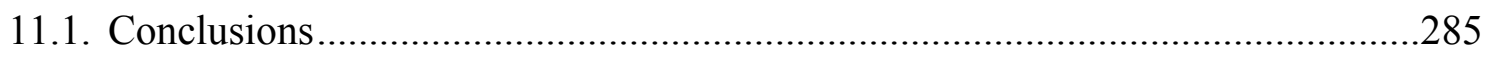

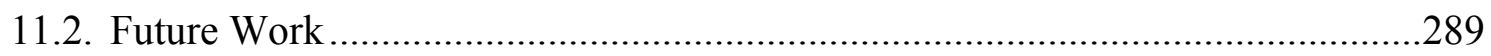

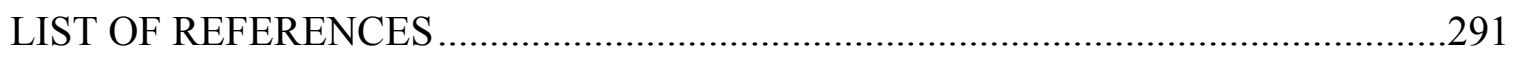

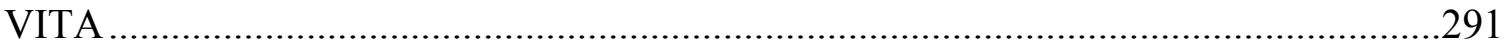




\section{LIST OF TABLES}

TABLE

PAGE

Table 2.1 U.S. Standard Plug-in Electric Vehicle Charging Levels ........................ 47

Table 5.1 Fuzzy Logic Algorithm Basic Process.............................................. 121

Table 5.2 Initial SOCs and Temperatures during Discharging ............................ 125

Table 5.3 Initial SOCs and Temperatures during Charging .............................. 126

Table 5.4 Battery Modules Droop Coefficients ................................................ 133

Table 6.1 Parameters For Phevs In Different Size.............................................. 144

Table 6.2 Arrival And Departure Times Distribution Parameters ........................ 145

Table 6.3 Charging Rates for Different Charging Levels.................................. 150

Table 6.4 Fuzzy Logic Rules ........................................................................ 153

Table 7.1 Parameters for PEVs In Different Size ............................................... 182

Table 7.2 Arrival And Departure Times Distribution Parameters ........................ 183

Table 7.3 Charging Rates for Different Charging Levels.................................. 189

Table 8.1 Specifications and parameters for Bidirectional DC-DC Converter ...... 212

Table 8.2 Specifications and parameters for Bidirectional AC-DC Inverter Active and Reactive Power Decoupled Control Simulation .................................. 224 


\section{LIST OF FIGURES}

FIGURE

PAGE

Figure 1.1 U.S. 2013 primary energy consumption by source and sector ................. 3

Figure 1.2 Renewable energy sources and generators ..................................... 8

Figure 1.3 Home scale hybrid power system with PEV ..................................... 14

Figure 1.4 Medium scale public PEVs hybrid parking garage power system .......... 15

Figure 2.1 Hybrid AC-DC power system configuration.................................... 42

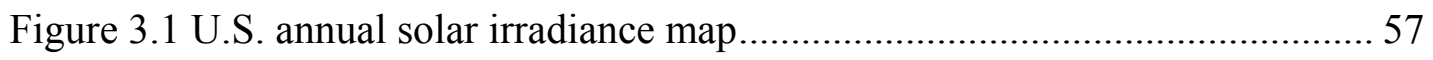

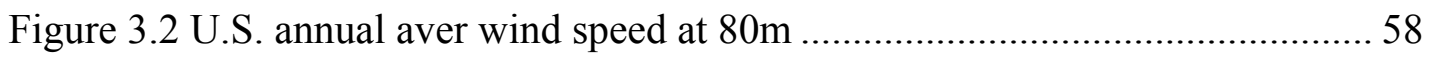

Figure 3.3 Florida annual average wind speed at $80 \mathrm{~m}$.................................... 59

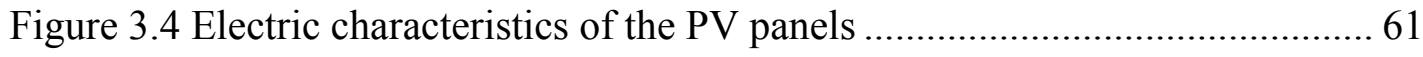

Figure 3.5 Equivalent circuit of a PV panel with a load.................................... 61

Figure 3.6 SunPower SPR-305-WHT solar cell P-V and I-V electric

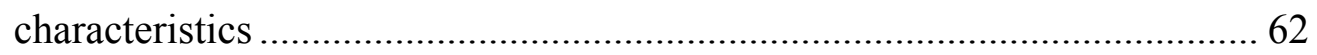

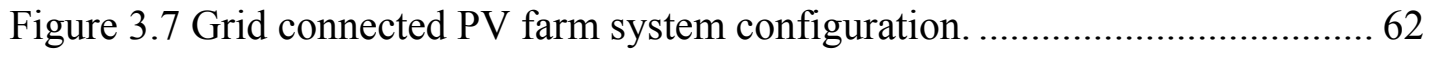



Figure 3.9 Circuit configuration during different states of the power electronic switch: (a) During On state $(0<\mathrm{t}<\mathrm{DT})$, (b) During OFF state $(\mathrm{DT}<\mathrm{t}<\mathrm{T}) \ldots . . .64$

Figure 3.10 Perturbation and observe MPPT operating point path. ....................... 67

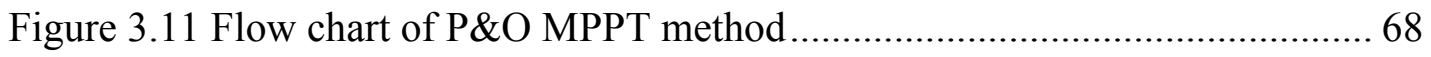

Figure 3.12 Block diagram for the proposed PV Emulator ................................. 70

Figure 3.13 DC-DC boost converter hardware ................................................ 70

Figure 3.14 Wind turbine rotation versus power characteristic at different wind



Figure 3.15 Basic diagram of doubly fed induction generator with back-to-back

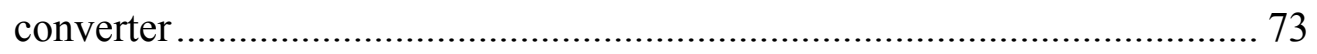


Figure 4.1 Overview of the proposed hybrid power system ............................... 85

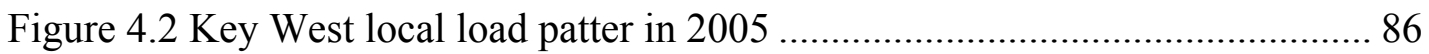

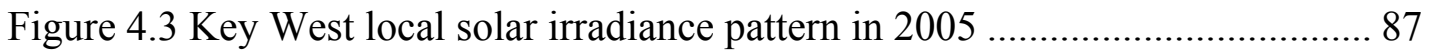

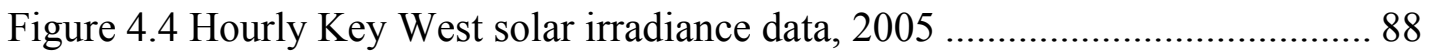

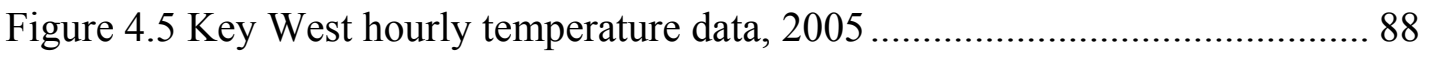

Figure 4.6 Key West local offshore wind speed pattern in the year 2005 ............... 89

Figure 4.7 Key West hourly temperature data, 2005 ............................................. 90

Figure 4.8 Renewable energy farm scale genetic algorithm optimization process... 93

Figure 4.9 Daily energy difference between the renewable energy farm output

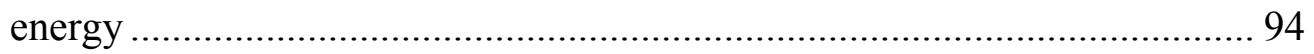

Figure 4.10 The one year minutely power difference between the renewable energy farm output and $20 \%$ of the local load .......................................... 96

Figure 4.11 Separation of low and high frequency power for battery and ultracapacitor support.

Figure 4.12 Power difference in one day period and its high frequency and low frequency parts.

Figure 4.13 The power difference between the renewable energy farm output and $20 \%$ of the local load with optimized energy storage system. 104

Figure 5.1 Over charged lithium-ion battery cell 111

Figure 5.2 Over heat impact to lithium-ion battery cell.

Figure 5.3 (a) lithium-ion battery pulse discharging response (b) lithium-ion battery equivalent circuit 113

Figure 5.4 Flow chart of the real time SOC and thermal monitoring..... 117

Figure 5.5 Lithium-ion battery bank ............................................................ 118

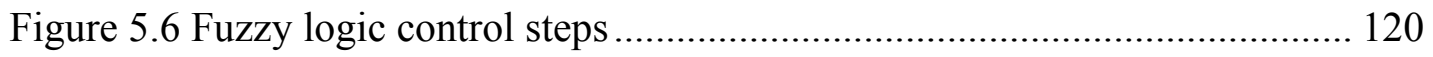

Figure 5.7 (a) $\Delta \mathrm{SOC}_{\mathrm{b}}$ membership function. (b) $\Delta T_{b}$ membership function. (c) $q_{n b}$ membership function. (d) Fuzzy logic controller rule surface. 123 
Figure 5.8 Lithium-ion battery bank SOC and temperature self-balancing circuit 124

Figure 5.9 SOC Balancing during Discharging Cycle......................................... 126

Figure 5.10 Temperature Balancing during Discharging Cycle .......................... 127

Figure 5.11 SOC Balancing during Charging Cycle ....................................... 128

Figure 5.12 SOC Balancing during Charging Cycle ......................................... 129

Figure 5.13 (a) $P-\omega$ droop characteristic. (b) $Q-V$ droop characteristic ............... 131

Figure 5.14 Hybrid power system DC subsystem configuration.......................... 132

Figure 5.15 Voltage-current droop characteristics of each battery module........... 134

Figure 6.1 PHEVs carpark hybrid powers system schematic diagram.................. 140

Figure 6.2 The PDF of the daily travel distance ............................................. 144

Figure 6.3 The PDF of the daily parking duration............................................ 145

Figure 6.4 Power needed by each PHEV when connected to the parking garage.. 146

Figure 6.5 The flow chart of the developed real time fuzzy logical charging controller. 148

Figure 6.6 Flow chart of Fuzzy Logic based controller for maximizing the utilization of power from PV

Figure 6.7 Membership functions and surface of the logic controller's rules base.

(a) Power flow; (b) Energy price; (c) Power flow control index, (d)

Surface of the fuzzy logic controller's rules base.

Figure 6.8 PHEVs charging power without real-time optimal charging control.... 156

Figure 6.9 PHEVs charging power with real-time optimal charging control ......... 157

Figure 6.10 Hourly power flow from AC grid without optimal controller............ 159

Figure 6.11 PHEVs' SOCs at their departure time without optimal controller..... 160

Figure 6.12 Hourly power flow from the AC grid with optimal controller............ 161

Figure 6.13 PHEVs' SOCs at their departure time with optimal controller.......... 162

Figure 6.14 PHEV's SOC change during the smart charging process. .................. 163 
Figure 6.15 69-Bus Radial Distribution Test Feeder. 165

Figure 6.16 Florida's normalized summer and winter daily load curves 166

Figure 6.17 (a) 69-Bus daily voltage profile with no PHEVs for summer load (b) 69-Bus daily voltage profile with no PHEVs for winter load. 167

Figure 6.18 PHEVs daily load profile in with no optimization and different

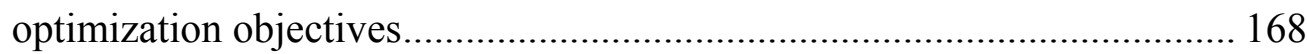

Figure 6.19 Daily voltage profile without real-time energy management algorithm for summer load. 169

Figure 6.20 Daily voltage profile without real-time energy management algorithm for winter load.

Figure 6.21 Daily voltage profile with real-time energy management algorithm for summer load.

Figure 6.22 Daily voltage profile with real-time energy management algorithm for winter load.

Figure 6.23 Daily voltage profile with real-time energy management algorithm considering energy function and summer load curve

Figure 6.24 Daily voltage profile with real-time energy management algorithm considering energy function and winter load curve.

Figure 7.1 Secondary control power reserve VS system power.

Figure 7.2 Overview of the proposed PEVs network charging system with renewable resources

Figure 7.3 PEVs charging hybrid AC-DC power system

Figure 7.4 South Florida local load and frequency pattern: (a) load pattern of the year, (b) summer load pattern, (c) winter load pattern, (d) frequency regulation pattern.

Figure 7.5 Historical wind data for one day and one month. 186

Figure 7.6 Renewable energy farm scale optimization process for PEV network charging by using GA. 
Figure 7.7 The flowchart of the developed real time fuzzy logical charging controller 189

Figure 7.8 (a) Surface of the fuzzy logic controller's rules; (b) Power flow membership function; (c) frequency regulation membership function; (d) Power flow control index membership function.

Figure 7.9 Charging of PEV network without smart charging algorithm. 195

Figure 7.10 Charging of PEV network with smart charging algorithm. 196

Figure 7.11 24-hour PEV network income 197

Figure 7.12 One year PEV network daily incomes. 198

Figure 8.1 Hybrid AC-DC micro grid configurations. 204

Figure 8.2 Bidirectional AC-DC inverter frequency regulation enable program ... 208

Figure 8.3 Topology of the bidirectional DC-DC converter. 209

Figure 8.4 Bidirectional DC-DC converter current control program. 210

Figure 8.5 Bidirectional DC-DC converter switches chosen circuit 211

Figure 8.6 Simulation of bidirectional DC-DC converter current control response212 Figure 8.7 Bidirectional DC-DC converter hardware circuits. 213

Figure 8.8 Bidirectional DC-DC converter current control experimental result.... 214

Figure 8.9 Bidirectional AC-DC inverter topology 216

Figure 8.10 Simplified model of the bidirectional AC-DC inverter 217

Figure 8.11 Bidirectional AC-DC inverter power control schematic diagram....... 220

Figure 8.12 Matlab simulation model for bidirectional AC-DC inverter power flow control. 221

Figure 8.13 Bidirectional AC-DC converter active and reactive power decoupled control 223

Figure 8.14 Bidirectional three phase AC-DC inverter hardware setup. 225

Figure 8.15 Bidirectional AC-DC inverter hardware active power control. (a) $I_{d}$ control (b) phase A voltage and current response. 227 
Figure 8.16 Bidirectional AC-DC inverter hardware reactive power control. (a) $I_{q}$ control (b) phase A voltage and current response............................... 228

Figure 8.17 Hybrid AC-DC power system AC subsystem frequency ................... 229

Figure 8.18 Power injected from DC subsystem to AC subsystem ...................... 229

Figure 8.19 PV farm output power control with MPPT. ................................ 231

Figure 8.20 Voltage control block diagram for bidirectional DC/DC converter .... 233

Figure 8.21 DC bus voltage control block diagram for bidirectional AC-DC

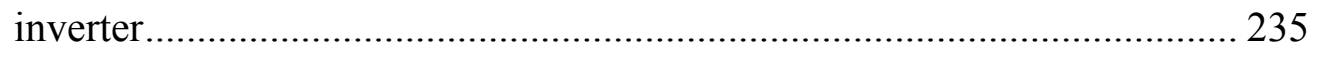

Figure 8.22 AC bus voltage control block diagram for bidirectional AC-DC

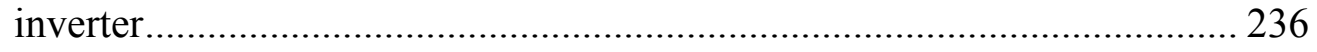

Figure 8.23 Hybrid AC-DC power system performances in grid-connected mode. 238

Figure 8.24 Hybrid AC-DC power system performances in grid-connected mode 239

Figure 8.25 Microgrid AC bus voltage and current response with DC support. .... 240

Figure 8.26 Hybrid AC-DC power system AC subsystem pulse load response with DC support.

Figure 8.27 AC subsystem slack generator performance under pulse load (a) output voltage, (b) output current. 242

Figure 8.28 AC subsystem frequency response 243

Figure 9.1 PEVs hybrid power system emulator with remote communication and control 246

Figure 9.2 Matlab Simulink bidirectional power flow control program..... 250

Figure 9.3 Three phase bidirectional AC-DC inverter data acquisition Matlab block 250

Figure 9.4 PLL and d-q transformation block. 251

Figure 9.5 Three phase AC-DC inverter active and reactive power decouple control block. 
Figure 9.6 Three phase bidirectional AC-DC inverter IGBT duty cycle

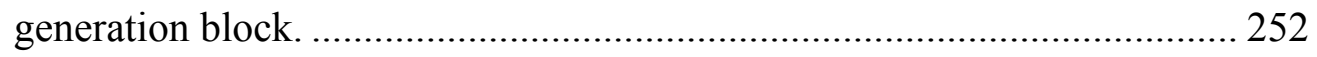

Figure 9.7 STM32F4 DISCOVERY microcontroller ......................................... 254

Figure 9.8 Signal converting circuit for STM32 (a) circuit hardware (b) circuit

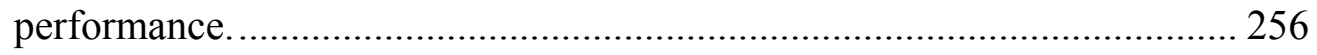

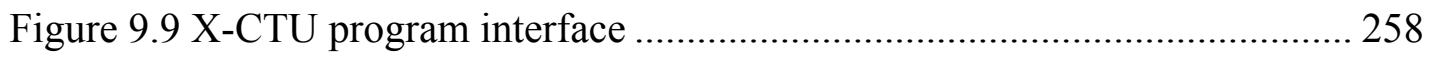

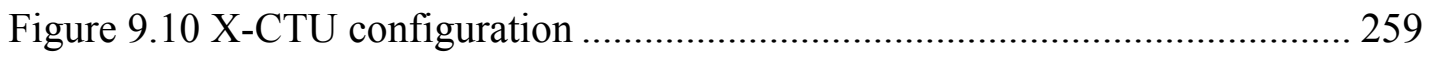

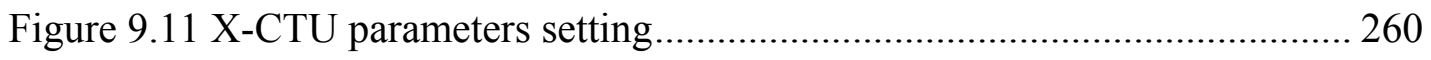

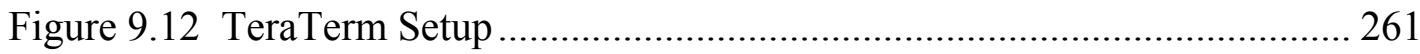

Figure 9.13 Battery banks SOC monitoring web server interface........................ 263

Figure 9.14 Bidirectional DC-DC converter PWM control program .................... 264

Figure 9.15 Zigbee parameters for STM32F microcontroller. ........................... 265

Figure 9.16 UART Rx1 block parameters................................................... 266

Figure 9.17 Bidirectional DC-DC converter PWM generation block. .................. 267

Figure 9.18 Battery banks monitor and control system.................................. 268

Figure 9.19 TeraTerm User Terminal .......................................................... 269

Figure 10.1 Laboratory based PEV car park emulator hybrid power system

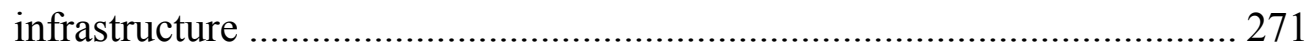

Figure 10.2 Circuit diagram of the grid connected bidirectional AC-DC inverter with filter in the proposed hybrid power system................................... 273

Figure 10.3 Lithium-ion battery module....................................................... 274

Figure 10.4 PEV car park emulator DC micro grid......................................... 275

Figure 10.5 Adaptive droop control program for multiple battery banks charging

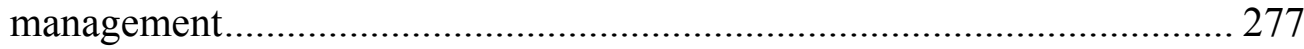

Figure 10.6 Flowchart of the battery banks smart energy management control

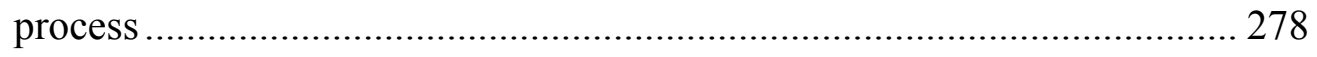

Figure 10.7 Hardware configuration of one PEV emulator ............................... 279 
Figure 10.8 Hybrid AC-DC power system hardware platform configuration........ 280

Figure 10.9 Hybrid AC-DC power system performance under pulse load and slack generator disconnection disturbances: (a) system power flow, (b) system frequency response............................................................... 281

Figure 10.10 Hybrid AC-DC power system performance under pulse load with DC subsystem support: (a) system power flow, (b) system frequency response.

Figure 10.11 Hybrid AC-DC power system performance under pulse load without DC subsystem support: (a) system power flow, (b) system frequency response. 


\section{LIST OF ACRONYMS}

$\begin{array}{ll}\text { ACRONYM } & \text { DETAILS } \\ \text { PEV } & \text { Plug-in Electric Vehicle } \\ \text { PHEV } & \text { Plug-in Hybrid Electric Vehicle } \\ \text { PLL } & \text { Phase Locked Loop } \\ \text { PV } & \text { Photo Voltaic } \\ \text { MPPT } & \text { Maximum Power Point Tracking } \\ \text { DSP } & \text { Digital Signal Processor } \\ \text { SOC } & \text { State of Charge } \\ \text { SOH } & \text { State of Health } \\ \text { PDA } & \text { Artificial Neural Network } \\ \text { SPWM } & \text { Sinusoidal Pulse Width Modulation } \\ \text { V2V } & \text { Venewable Energy Resources } \\ \text { V2G } & \text { Vehicle to Grid } \\ & \end{array}$




$\begin{array}{ll}\text { FLC } & \text { Fuzzy Logic Control } \\ \text { PID } & \text { proportional-integral-derivative controller } \\ \text { GHG } & \text { Greenhouse Gas } \\ \text { NREL } & \text { National Renewable Energy Laboratory } \\ \text { STATCOM } & \text { Static Synchronous Compensator } \\ \text { RT-SLM } & \text { Real-time Smart Load Management } \\ \text { HVDC } & \text { High Voltage DC } \\ \text { LED } & \text { Light-Emitting Diodes } \\ \text { FPL } & \text { Florida Power \& Light } \\ \text { PWM } & \text { Pulse-Width Modulation } \\ \text { IGBT } & \text { Insulated-Gate Bipolar Transistor } \\ \text { P\&O } & \text { Perturbation and Observe } \\ \text { GUI } & \text { Graphic User Interface } \\ \text { DFIG } & \text { Noubly-Fed Induction Generator } \\ \text { LPF } & \text { Low Pass Filter } \\ \text { HPF } & \text { Normal Operating Cell Temperature } \\ \text { NOCT } & \end{array}$




\section{Introduction}

\subsection{Background}

The electric power system, which includes the power generation, transmission and distribution is the most complex system that human beings have built. Based on U.S. Energy Information Administration statistics, present U.S. annual electricity generation and consumption is more than 13 times greater than it was in the 1950 s. Despite the increasing generation and consumption, the majority of the current U.S. power grid was built more than 80 years ago [1]. The rising demand of the electricity consumptions especially the pulse ones that may absorb a huge amount of energy in short period will put the old power system infrastructure under enormous pressure [2].

The general power systems structure can be classified as three major parts: energy generation units, which produce electrical power from other sources of energy; transmission networks, which transfer electricity from the generation units to local substations; and distribution systems, which supply the electrical power to the terminal residential, commercial and industrial customers [3]. Based on the U.S. Energy Information Administration annual energy review, in 2014, more than $80 \%$ of the energy consumed in the USA was generated by petroleum, natural gas and coal. This means present electricity generation is still heavily dependent on environmentally costly fossil energy [4]. At the same time, the exhaustion of the global energy reserves is already a worldwide problem at the environmental, industrial, economic, and societal levels. What 
is worse, the present U.S. transmission and distribution system loses an average of $7 \%$ of the total annual electricity generation for 2014. Based on the average national energy retail price, annual losses for the whole country were amount to nearly $\$ 30$ billion. At the same time, renewable energy sources supplied no more than $8 \%$ of the total energy consumption [5]. Over the last few decades, global temperatures have been rising due to the accumulation of greenhouse gases, which may result in serious environmental changes and raise sea levels [6]. Due to this, many countries have made the reduction of greenhouse gas emissions a major governmental goal and have taken actions to minimize the emissions.

Two key solutions to reduce the greenhouse gas emissions are to maximize the utilization of renewable energy resources to generate energy for load consumption [7] and to shift to low or zero emission vehicles for transportation [8]. Based on the 2013 U.S. primary energy consumption by source and sector report as shown in figure 1.1, the transportation sector is responsible for $29 \%$ of the total annual energy consumption. Therefore, the electrification of vehicles and charging those plug-in electric vehicles (PEVs) with the energy generated from renewable energy farms in hybrid AC-DC power systems has become the best option for reducing greenhouse gas emission. The U.S government has set itself the goal that by the fiscal year 2020, assuming economic feasibility and to the extent being technically practicable, 20 percent of the total amount of electric energy consumed by each agency during any fiscal year thereafter shall be renewable energy [9]. However, the existing power grid network topology is feeble for high penetration of renewable energy sources and PEVs which may bring a significant impact on the performance and reliability of the electricity grid [10]-[11]. This is largely 
Primary Energy Consumption by Source and Sector, 2013

(Quadrillion Btu)
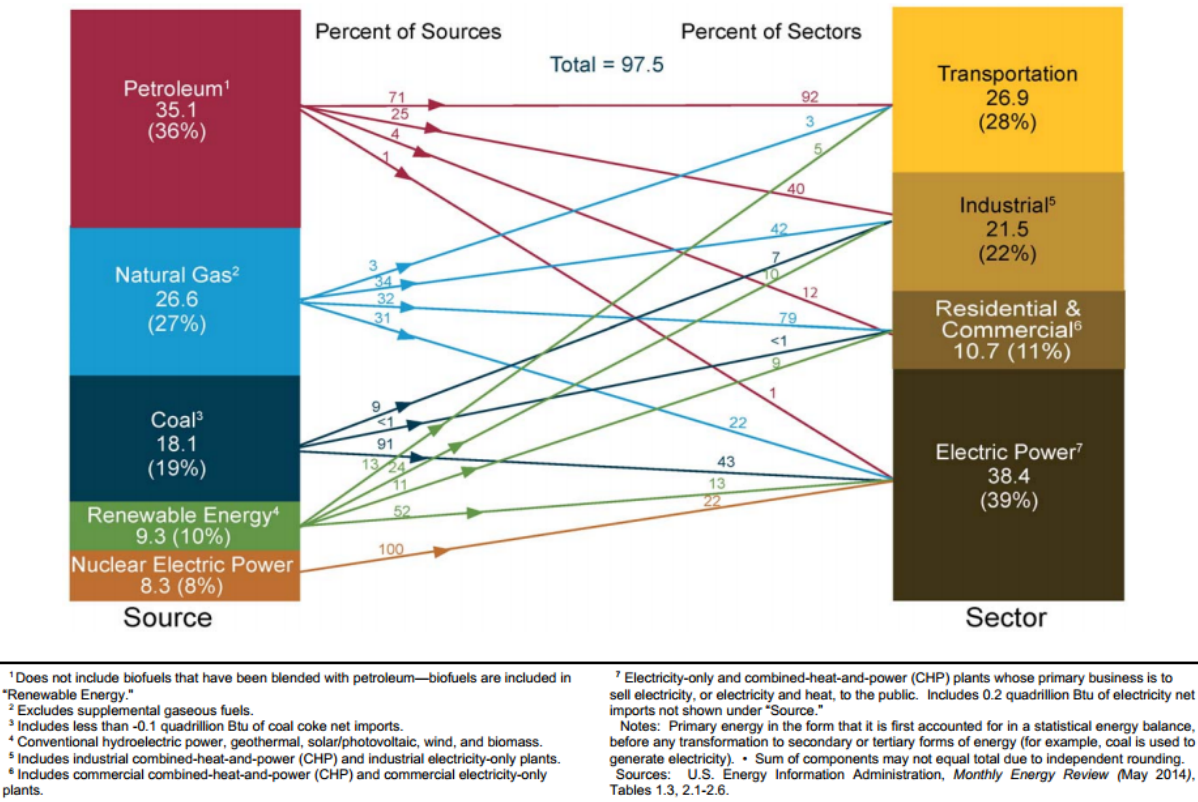

Figure 1.1 U.S. 2013 primary energy consumption by source and sector

because of the variability of renewable resources and the lack of large-scale economical storage capability. Therefore, there is an urgent need to reconstruct the U.S. power grid system to smartly enhance the energy transmission and distribution capability, while at the same time increasing the whole efficiency to reducing the energy loss.

Currently, there is insufficient research available for planning, designing and constructing of hybrid AC-DC power systems with renewable energy sources and PEVs. Due to the large variances of renewable energy sources, the power generated from a renewable farm consisting of PV or wind turbines is considered intermittent. Therefore, without properly planning the scale of the renewable energy farm, the utility grid may be badly influenced by the power generated by the renewable energy farm. Using energy storage devices as an energy buffer is a solution to limit the renewable energy farm impact limit to the utility grid [12]. Unfortunately, the present market prices of the energy 
storage devices, especially the ones with high energy density such as lithium-ion battery or high power density such as ultra-capacitor, are very high. Therefore, the design and construction of renewable energy farms together with the energy storage systems need to be carefully planned and optimized to get the best cost performance.

Nowadays, lithium-ion batteries used as energy storage devices are playing an important role in power systems due to the growing popularity of micro grids with renewable energy sources and PEVs. The performance of the hybrid AC-DC power system and PEVs strongly relies on their battery bank management systems, which always consist of multiple cells connected together in series and/or in parallel. The energy conversion efficiency of the battery bank system is influenced by state of charge (SOC) and temperature variations of each single battery cells. Thermal and SOC imbalances increase the risk of catastrophic faults in the battery bank system. The recent Boeing 787 Dreamliner's lithium-ion battery faults in Japan and Boston have illustrated the importance of developing reliable and efficient battery management systems in high energy density battery systems [13]. The specific characteristics and needs of the smart hybrid AC-DC power system and PEVs, such as deep charge/discharge protection and accurate SOC and state-of-health (SOH) estimation, intensify the need for a more efficient and reliable battery management system [14].

Meanwhile, without proper control, even with low penetration, the PEVs charging process may bring huge impact and create potential risk to the existing power grid system. Due to the expectation that the PEVs are more likely to be connected to the utility grid in specific time periods, the uncontrolled charging may tremendously increase the daily load peak, which will also increase the power flow in the transmission and 
distribution lines and degrade the power system stability and reliability [15],[16]. On the other hand, if smartly regulated, the PEVs can serve as distributed energy storage devices when they are parked in the garage for a long time period. This can help the local grid relieve the peak demand and keep the real-time balancing of power. What's more, with the Vehicle-to-Grid (V2G) and Vehicle-to-Vehicle (V2V) technologies [17], PEVs batteries can feed electricity back to the AC power grid to help the system keep the frequency and voltage amplitude stable.

Based on the situation mentioned above, there is a trend to change the current old power grid system into smart and distributed controlled hybrid power systems involving both AC and DC distributed architectures to improve of the whole system reliability, stability, efficiency, sustainability, robustness, security and decrease complexity and cost. The smart hybrid power system should have features that can be summarized as follows [18]-[27]:

1. Distributed assets, information and control;

2. Optimized with all resources and subsystems;

3. Self-healing and self-balancing;

4. Predictive to oncoming events and prevent emergencies;

5. Resilient to cyber physical attacks;

6. Interactive with customers, utility grid and market;

7. Robust to critical loads and disturbance;

8. Friendly interface with plug and play function. 
In this dissertation, an initiative is taken to have a deeper study of the hybrid ACDC power system smart operation involving high penetration of renewable energy sources and PEVs, with whole system modeling, forecasting, optimization, protection and control. This dissertation mainly addresses two technical issues associated with hybrid power systems: hybrid power system operation with integration of distributed renewable energy sources; and hybrid power system operation and protection with plug-in electric vehicles and critical loads.

\subsection{Current State of Hybrid Power Systems}

Hybrid AC-DC power systems incorporate several electricity generating components in both AC and DC sides with usually one major generator controls system and enables the system to supply electricity in the required quality. Components for electricity generation can utilize renewable energy sources like wind turbines, photovoltaic, solar thermal, hydro power, wave power or biomass power stations, etc. Furthermore, fossil power plants like diesel generators, gas turbines or fuel cells, etc. can also be added [28].

The term hybrid AC-DC power system does not give any information about the size of the power system. Generally, hybrid AC-DC power systems are considered to supply loads in the size of several watts up to several megawatts. Hybrid AC-DC power systems can be connected to a large utility grid and operated as grid-connected mode. They can also supply island networks and represent small grids with a limited number of consumers. Due to the resulting fluctuating consumption pattern several specific features are required concerning the electricity supplying hybrid AC-DC power systems. 
In utility grids, the power equalizes due to the large number of consumers and its statistical application. This is how base load, medium load and peak load are defined, which are covered by dedicated base, medium and peak load power plant to minimize the electricity cost. Base load is needed continuously 24 hours per day, medium load is required in consecutive 3 to 6 hours periods, and peak load is required in shorter sequences [29]. Unfortunately, this cost effective procedure cannot be transferred to hybrid power systems in most of the cases when it is operated in islanding mode. On the other hand, hybrid AC-DC power systems have to cope with much more severe short term variations in power demand and renewable sources energy output. Thus, smart and efficient energy management structures have to be applied to maintain the system stability [30]. These energy management structures vary with the size of the hybrid ACDC power system depending on financially optimal system design.

When operated in islanded mode, it is essential to integrate one energy generator that is responsible for the AC side frequency and voltage stabilization. At the same time, the DC side voltage also needs to be regulated by an energy source [31]-[33]. In small hybrid AC-DC power system of up to $50 \mathrm{~kW}$, inverters and energy storage systems can be applied for frequency and voltage stabilization. In lager systems, continuously running synchronous generators with controllable engines are more cost effective. For future Megawatt class hybrid AC-DC power systems with high penetration of PEVs, the frequency and voltage can be regulated through V2G services [34].

\subsubsection{Hybrid power system with renewable energy sources}

Energy sources such as wind, solar, biomass, hydro, tide and geothermal energy are generally recognized as renewable energy because they are inexhaustible or can be 
regenerated. Figure 1.2 shows different types of renewable energy sources and generators. The overview of the commonly utilized renewable energy sources can be found in [35]-[38].

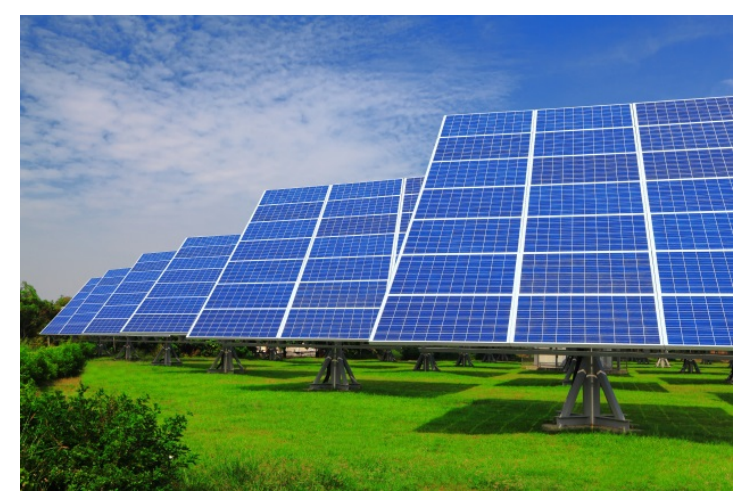

Solar Energy

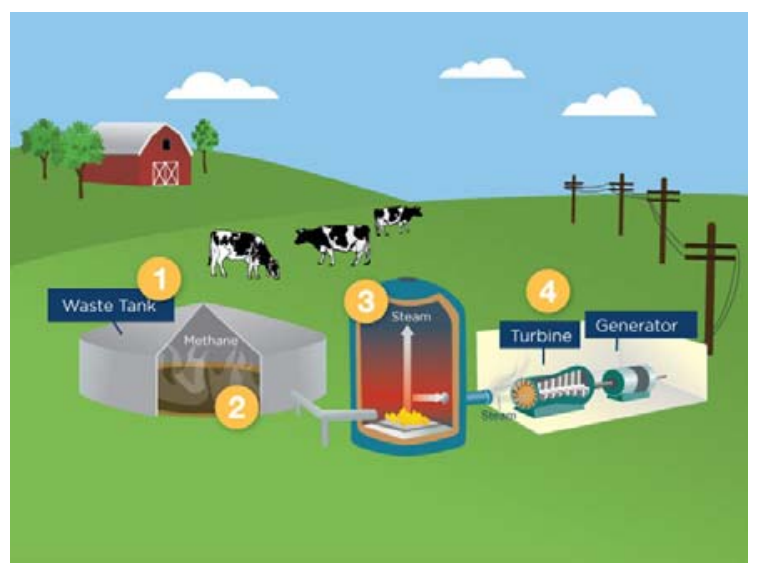

Biomass Energy



Geothermal Energy

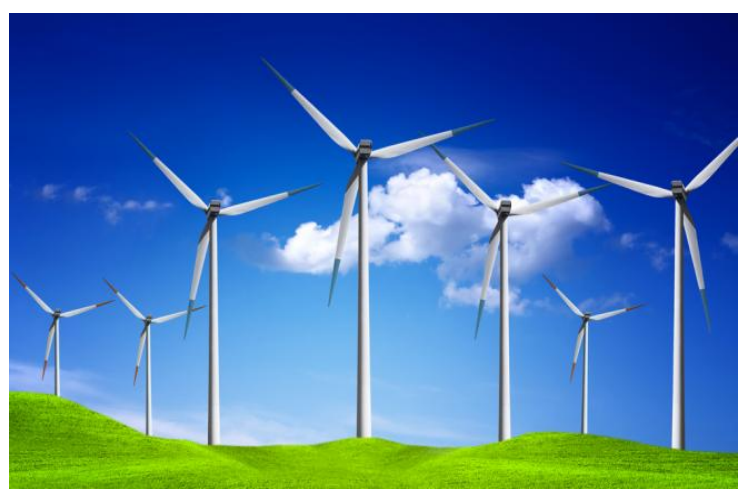

Wind Energy

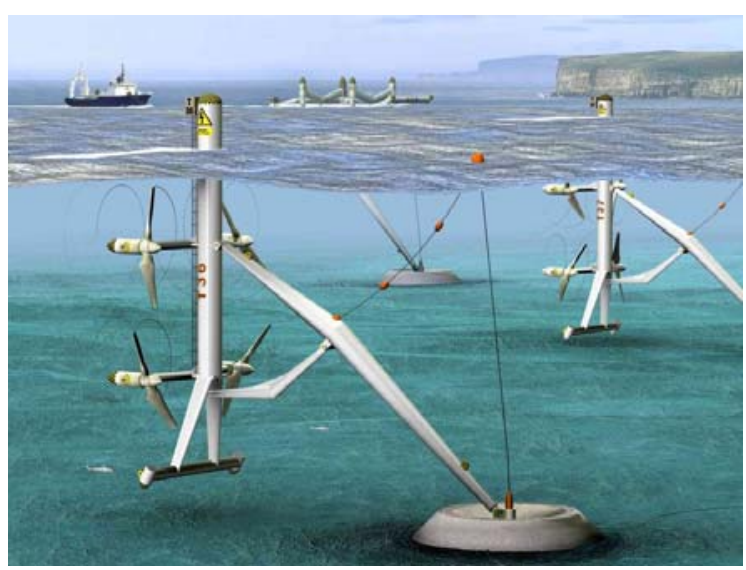

Tide Energy

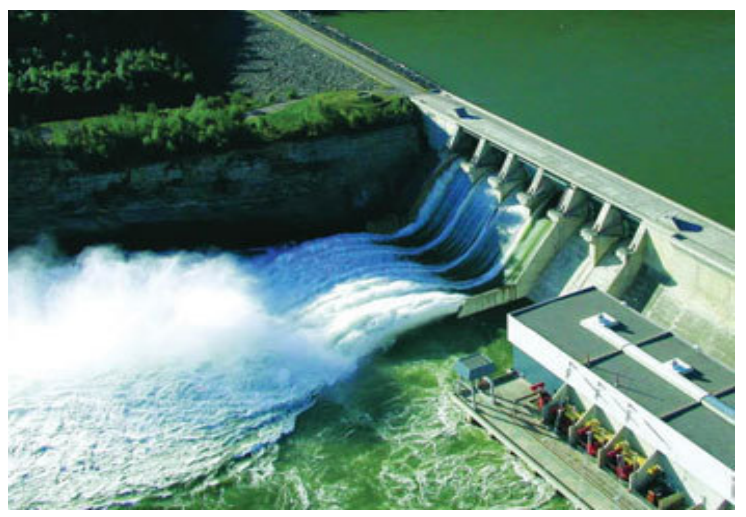

Hydro Energy

Figure 1.2 Renewable energy sources and generators 
The utilization of renewable energy sources, such as photovoltaic panels, wind turbines and fuel cells are becoming more and more popular. Therefore, in the upcoming future, the penetration level of those renewable energy sources will keep raising in the U.S. utility grid systems. Some of the renewable energy generation systems like photovoltaic panel and fuel cells generate a DC output voltage. Therefore they can be connected to the DC side of the hybrid AC-DC power system. Some other renewable energy generation systems such as wind turbines, hydro energy generators and tide energy generators generate an AC output voltage. Those systems can be connected either to the DC side of the hybrid power system through a power rectifier or to the AC side of the hybrid power system.

Among those different renewable energy resources, solar and wind are the most popular and have been utilized for centuries by human beings because they are the most sufficient energy sources and are easy to be harvested.

Solar technologies are broadly characterized as either passive solar or active solar depending on the way they capture, convert and distribute solar energy. To harvest the solar energy, the most common way is to use photovoltaic panels [39]. In a photovoltaic system, solar irradiance is directly converted into electricity with solar cells, which are produced from wafers or fine films. Those semiconductor devices can convert solar irradiance into DC current. The photovoltaic panel efficiency can reach up to $30 \%$, depending on the materials, design technology, solar irradiance, and temperature.

Wind energy is derived from the solar energy because the earth is unevenly heated by the sun. For example, the areas near the equator receive more energy from the sun than the areas next to the poles. The different temperatures drive the movement of air 
and generate wind. Wind power can be harvested by using wind turbines and converted to either electrical power or mechanical power. Wind power capacity has expanded rapidly to $336 \mathrm{GW}$ as of June 2014 . At present wind energy production is around $4 \%$ of total worldwide electrical production, and growing rapidly [40]. On an annual basis, as of 2011, few grid systems have penetration levels above 5\%: Denmark 39\%, Portugal 19\%, Spain $19 \%$, Ireland $18 \%$, and Germany $11 \%$. For the U.S. in 2011 , the penetration level was estimated at $3.3 \%$. Large scale wind farms can be connected to the electric power transmission network and serve as big energy generators. Small wind farms can be connected to both the AC and DC sides of the hybrid power system, in both grid connected mode and islanding mode.

To obtain high penetration in hybrid AC-DC power systems from wind and solar energy requires substantial long term storage or substantial interconnection to other systems which may already have substantial storage together with power filters to enhance the power quality.

\subsubsection{Plug-in electric vehicles charging in hybrid power system}

As mentioned in section 1.1, the transportation sector consumed nearly $30 \%$ of the total U.S. energy consumption. To accelerate the utilization of renewable and green energy at the same time reduce the greenhouse gas emissions, one potential solution is to replace traditional gasoline vehicles with PEVs.

A PEV is any motor vehicle that can be recharged from an external source of electricity, such as wall sockets, and the electricity stored in the rechargeable battery packs drives or contributes to drive the wheels. PEV is a superset of electric vehicles that includes all-electric or battery electric vehicles, plug-in hybrid vehicles, and electric 
vehicle conversions of hybrid electric vehicles and conventional internal combustion engine vehicles.

Recently, PEVs are becoming more and more popular because PEVs have several benefits compared to conventional internal combustion engine vehicles. They have lower operating and maintenance costs, and produce less or even no local air pollution. They reduce dependence on petroleum and may reduce greenhouse gas emissions from the onboard source of power, depending on the fuel and technology used for electricity generation to charge the batteries. PEVs capture most of these benefits when they are operating in all-electric mode.

Several national and local governments have established tax credits, subsidies, and other incentives to promote the introduction and adoption in the mass market of PEVs depending on their battery size and all-electric range. As of October 2014, there are over 45 models of highway legal PEVs available for retail sales. Global sales increased from 45,000 units in 2011 to 206,000 in 2013. As of September 2014, more than 600,000 highway-capable PEVs and light utility vehicles have been sold worldwide, with the United States as the market leader with about 260,000 units delivered since 2008, representing $43.0 \%$ of global sales. Japan ranks second with over 95,000 units sold since 2009 (15.8\%), followed by China with more than 77,000 units sold since $2010(12.8 \%)$. Over 163,000 plug-in electric vehicles have been sold in the European market as of September 2014, representing $27.0 \%$ of global sales. Sales are led by the Netherlands with about 41,000 units registered, followed by France with almost 39,000 all-electric cars and light utility vans sold since 2010, and Norway with about 38,00 plug-in electric vehicles registered. The Nissan Leaf is the world's top selling highway-capable all- 
electric car, with global sales of over 150,000 units by November 2014, followed by the Chevrolet Volt plug-in hybrid, which together with its sibling the Opel/Vauxhall Ampera have combined sales of more than 87,000 units as of November 2014.

Despite their potential benefits, market penetration of PEVs has been slower than expected as adoption faces several hurdles and limitations. The factors discouraging the adoption of electric cars can be summarized as follows [41]:

- Cost of lithium-ion batteries and cost of ownership [42]

As of 2013, plug-in electric vehicles are significantly more expensive as compared to conventional internal combustion engine vehicles and hybrid electric vehicles due to the additional cost of their lithium-ion battery pack.

- Rare earth metals availability and supply security

Common technology for PEVs is based on the lithium-ion battery and an electric motor which uses rare earth elements. The demand for lithium, heavy metals, and other specific elements (such as neodymium, boron and cobalt) required for the batteries and powertrain is expected to grow significantly due to the future sales increase of PEVs in the mid and long term.

- Risks associated with noise reduction

PEVs operating in all-electric mode at low speeds produce less roadway noise as compared to vehicles propelled by an internal combustion engine, thereby increasing the risk of accidents where pedestrians do not hear the vehicle coming.

- Availability of recharging infrastructure [43]

Despite the widespread assumption that PEVs charging process will take place overnight at home, residents of cities, apartments, dormitories, and townhouses do not 
have enough garages or driveways with available power outlets, and the customers might be less likely to buy plug-in electric vehicles unless recharging infrastructure is developed.

- Potential overload impact to the utility grid [44]

The present aging utility grid and local transformers in particular, may not have enough capacity to handle the additional power load that might be required in certain areas with high PEV concentrations. Recharging a single PEV can consume three times as much electricity as a typical home, so overloading problems may arise when several PEVs in the same neighborhood recharge at the same time, or during the normal daily peak loads.

- Risks of battery fire

Lithium-ion batteries may suffer thermal runaway and cell rupture if overheated or overcharged, and in extreme cases this can lead to combustion. When handled improperly, or if manufactured defectively, some rechargeable batteries can experience thermal runaway resulting in overheating. Lithium-ion batteries are especially prone to thermal runaway.

The hybrid AC-DC power system architecture is the best solution to solve the last three disadvantages of PEVs as mentioned above. Hybrid AC-DC power system is suitable for PEVs charging infrastructures with different scales.

For small home scale hybrid power systems with utility grid connection and DC voltage output chargers for PEVs as shown in figure 1.3, power generated by the renewable energy sources can be used to charge the PEVs. The hybrid power system is also connected to the utility grid, therefore when renewable energy is not sufficient, the 


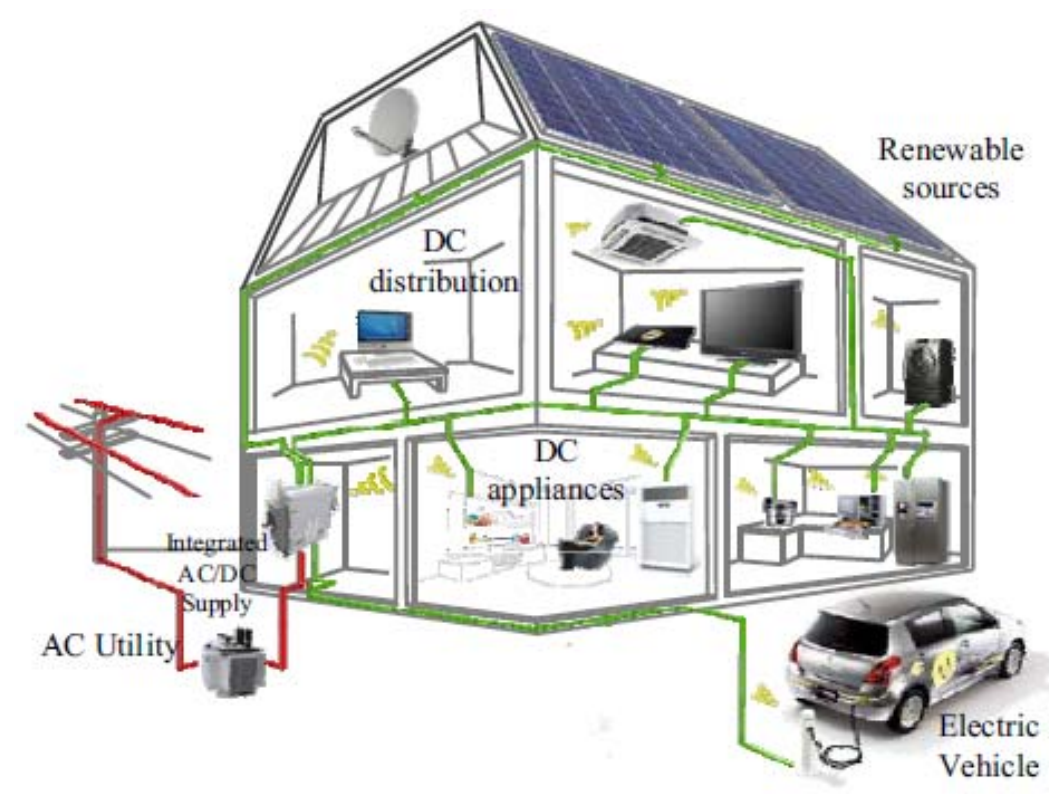

Figure 1.3 Home scale hybrid power system with PEV

PEVs still can be charged from the utility grid. What's more, the PEVs can be viewed as an uninterruptible power supply (UPS) for the hybrid power system and improve the performance of the DC power network and the household appliances inside it [45]-[48].

For medium scale public PEV hybrid parking garage power systems with several PEVs charging together from the same DC bus as shown in figure 1.4, power generated by the renewable energy farm associated with the parking garage can be used to charge the PEVs, which can greatly decrease the greenhouse gas emissions and limit the PEVs charging impact to the local utility grid connection point. Also, the parking garage hybrid power system can monitor the PEVs SOC and $\mathrm{SOH}$, and in some particular situations, the garage can transfer energy from one PEV to another through the V2V function instead of absorbing power from the utility grid with high price [49]-[51]. This will be especially helpful during the load peak period. In addition, the parking garage can be viewed as a huge energy storage with a large capacity. Therefore it can be used to improve the power 
quality of hybrid AC-DC power systems with critical loads such as medical apparatus and instruments in a hospital.

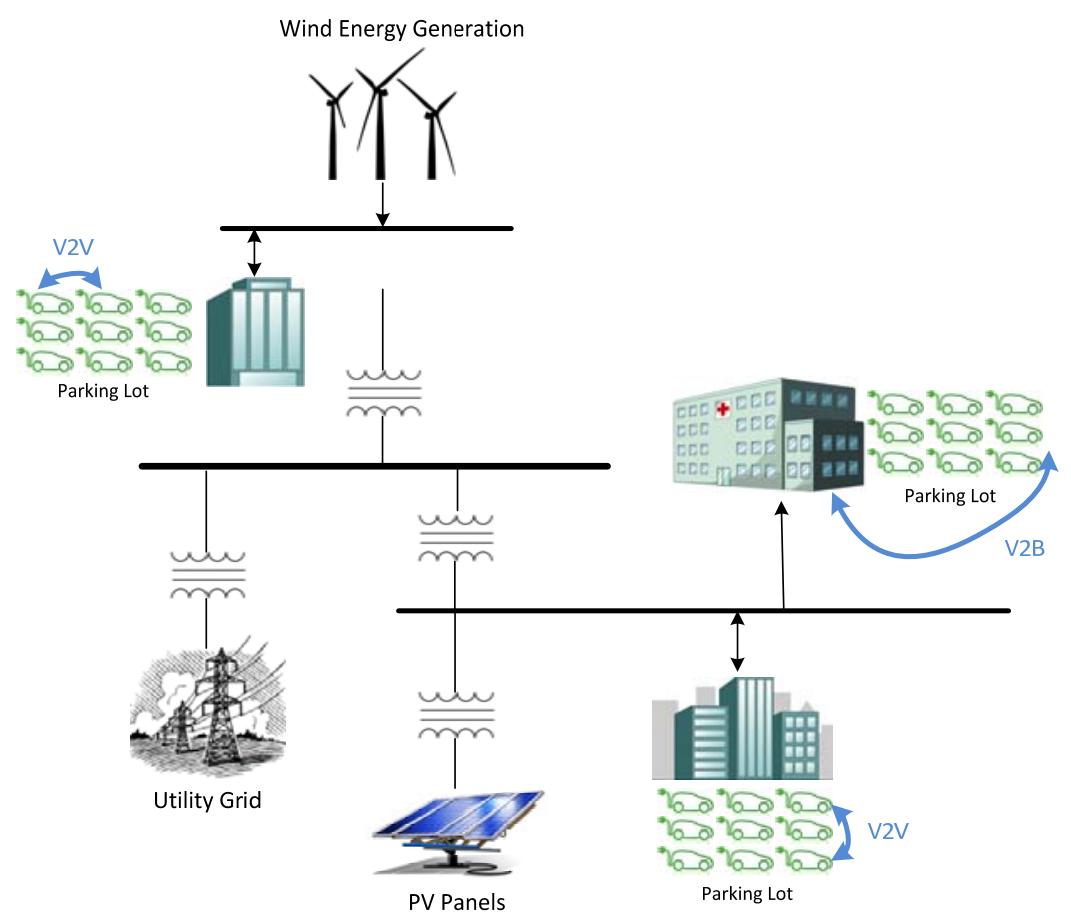

Figure 1.4 Medium scale public PEVs hybrid parking garage power system

For large scale PEV network hybrid power system in urban areas, the renewable energy farm power output can be more precisely forecasted. Also, because there are a huge numbers of PEVs in the network, a single PEV behavior won't influence the whole network overall energy requirement and charging pattern. Therefore, with proper planning and management, more energy can be utilized to charge the PEVs. Also, PEVs offer users the opportunity to sell electricity stored in their batteries back to the power grid, thereby helping utilities to operate more efficiently in the management of their demand peaks. A V2G system would take advantage of the fact that most vehicles are parked an average of 95 percent of the time. During such idle times the electricity stored in the batteries could be transferred from the PEV to the power lines and back to the grid 
[52]-[55]. In the U.S this transfer back to the grid would have an estimated value to the utilities of up to $\$ 4,000$ per car per year [56]. In a V2G system it would also be expected that PEVs would have the capability to communicate automatically with the power grid to sell demand response services by either delivering electricity into the grid or by throttling their charging rate.

In the hybrid AC-DC power system with PEVs, protections and limitations can be set to prevent the batteries in the PEVs from over charge/discharge and over heat issues. Also, with smart charging management and properly set communication network, the SOC and $\mathrm{SOH}$ of the batteries will be monitored, therefore the risks of battery fire can be completely eradicated and the life time of the battery banks will be extended [57]-[58].

\subsubsection{Lithium-ion battery in power system and plug-in electric vehicles}

Lithium-ion battery is a member of a family of rechargeable battery types in which lithium ions move from the negative electrode to the positive electrode during discharge and back when charging. Lithium-ion batteries use an intercalated lithium compound as one electrode material, compared to the metallic lithium used in a nonrechargeable lithium battery [59]. The electrolyte, which allows for ionic movement, and the two electrodes are the consistent components of a lithium-ion cell.

Lithium-ion batteries are common in consumer electronics. They are one of the most popular types of rechargeable batteries for portable electronics, with a high energy density, no memory effect, and only a slow loss of charge when not in use. Beyond consumer electronics, Lithium-ion batteries are also growing in popularity for military, PEVs and hybrid AC-DC power systems with renewable energy sources and critical loads. For example, lithium-ion batteries are becoming a common replacement for lead 
acid batteries that have been used historically for golf carts and utility vehicles. Instead of heavy lead plates and acid electrolyte, the trend is to use lightweight lithium-ion battery packs that can provide the same voltage as lead-acid batteries, so no modification to the vehicle's drive system is required.

However, lithium-ion batteries can be dangerous under some conditions and can pose a safety hazard since they contain, unlike other rechargeable batteries, a flammable electrolyte and are also kept pressurized. If overheated or overcharged, lithium-ion batteries may suffer thermal runaway and cell rupture [60]. In extreme cases this can lead to combustion. To reduce these risks, lithium-ion battery packs should contain protection circuit that disconnects the battery when its voltage is outside the safe range of 3-4.2 V per cell. Exceeding this voltage range results in premature aging of the cells and, furthermore, safety risks due to the reactive components in the cells. For lithium-ion battery banks with several lithium-ion cells connected in series and/or in parallel, the failure of one single cell because of over-charging/discharging or over heating may ruin the whole battery bank's performance. Therefore, the cells SOC and thermal monitor and balance circuit is essential to protect the lithium-ion battery bank and ensure it is operated at suitable conditions. The PEVs and hybrid AC-DC power system with lithium-ion battery banks need to take this into consideration [61].

\subsubsection{Artificial intelligence in hybrid power system}

Artificial intelligence is the intelligence exhibited by machines or software. The central goals of Artificial intelligence research include reasoning, knowledge, planning, and learning natural language processing (communication), perception and the ability to move and manipulate objects. General intelligence is still among the field's long term 
goals. Currently popular approaches include statistical methods, computational intelligence and traditional symbolic artificial intelligence. There are a large number of tools used in artificial intelligence, including versions of search and mathematical optimization, logic, methods based on probability and economics, classifiers and statistical learning methods and many others. The artificial intelligence field is interdisciplinary, in which a number of sciences and professions converge, including computer science, mathematics, psychology, linguistics, philosophy and neuroscience, as well as other specialized fields such as artificial psychology.

Artificial intelligence are widely applied to hybrid AC-DC power system in the areas of planning, design, optimization, forecasting, management, and control. Artificial neural network, genetic algorithm and fuzzy logic are three popular tools used in the application of pattern recognition and forecasting, design optimization, and nonlinear control, respectively.

Artificial neural networks (ANN) are a family of statistical learning algorithms inspired by biological neural networks (the central nervous systems of animals, in particular the brain) and are used to estimate or approximate functions that can depend on a large number of inputs and are generally unknown. ANN are generally presented as systems of interconnected "neurons" which can compute values from inputs, and are capable of machine learning as well as pattern recognition [62].

Due to uncertain factors such as the next period load and the upcoming environmental factor variations, the operation of a hybrid power system (especially the ones with high penetration of renewable energy sources, PEVs and critical loads) needs a precise forecasting to properly manage the generation, storage and distribution of energy. 
ANN is a good tool that can be utilized to forecast the short-term and long-term factors in hybrid AC-DC power systems such as the upcoming load, solar irradiance, temperature and wind speed. With this forecasted information, the hybrid power system can estimate the energy generation and consumption gap in the near future, properly distribute the available energy and maximize the utilization of the renewable energy sources.

Genetic Algorithm (GA) is a search heuristic that mimics the process of natural selection. This heuristic (also sometimes called a metaheuristic) is routinely used to generate useful solutions to optimization and search problems. Genetic algorithms belong to the larger class of evolutionary algorithms, which generate solutions to optimization problems using techniques inspired by natural evolution, such as inheritance, mutation, selection, and crossover [63].

A hybrid AC-DC power system is a complex and highly coupled system in which any component's tiny variation may generate huge system performance differences. In those hybrid power systems, most of the traditional optimization methods can't offer acceptable optimal solutions for system design and components chosen. GA is a powerful tool and can be widely applied in hybrid AC-DC power system optimizations, from the components level optimization such as optimizing inductor and capacitor sizes and positions in a bidirectional DC-DC converter to minimize the electromagnetic interference, to the system level optimization such as searching the optimized scales of the renewable energy farm and energy storage system to supply enough power for a certain amount of the local demand with best cost performance.

Fuzzy Logic Control (FLC) is based on fuzzy logic, a form of many-valued logic which deals with reasoning that is approximate rather than fixed and exact. 
Compared to traditional binary sets (where variables may take on true or false values), fuzzy logic variables may have a truth value that ranges in degree between 0 and 1 . Fuzzy logic has been extended to handle the concept of partial truth, where the truth value may range between completely true and completely false. Furthermore, when linguistic variables are used, these degrees may be managed by specific functions [64].

In hybrid AC-DC power systems, traditional controllers like proportionalintegral-derivative controller (PID controller) can handle most of the real-time regulation and adjusting issues such as controlling the power flow in power electronics devices [65]. But in some cases, the control plant doesn't have direct mathematic relationship with the inputs, such as the PEVs charging rates are not directly depend on the factors like the renewable energy farm output power, the AC grid frequency, the power grid frequency, the energy price, etc. In this situation, FLC is a useful tool to link those factors and manage the whole system.

\subsubsection{Information technology and wireless communication}

The smart management of hybrid power systems is highly dependent on communication infrastructures. Having effective communication technologies will be critical to the successful operation of a hybrid power system with multiple components and power electronic devices. In general, the communication networks can be categorized as home area network, field area network and wide area network [66]. Depending on the location of the hybrid AC-DC power system such as home or PEVs parking garage, the needed network architecture is related with home area network and field area network. The electrical sensors and smart meters on the PEV charging stations and renewable 
energy farms can monitor and exchange information with central aggregator through the field area network to smartly manage the power flow in the hybrid AC-DC power system.

The required two-way communication network and bi-directional power flow (charging/discharging) between the PEVs and the hybrid AC-DC power system may complicate the operations. Therefore a reliable communication network is needed to enable the successful integration of a large number of PEVs [67]-[68]. In a PEV car park garage, some special services must be offered such as: enroll, register, and set up initial communications with the hybrid power system; provide PEV charging status information to the hybrid power system, and regulate PEVs charging signals with the customers selected rates and energy price.

A variety of communication protocols can achieve reliable two-way communication networks. However, communication technology options are unproven yet for hybrid power systems with PEVs and renewable energy sources. So far, there is neither a well-defined industrial code nor a standard for communication between PEVs and the hybrid charging power station. Based on the specific communication needs of PEVs, HomePlug network, ZigBee network and Cellular Network are three popular communication network topologies that can be potential solutions to the future PEVs parking garage hybrid power system [69]-[71]. In this dissertation, ZigBee network is chosen in the PEV parking garage hybrid power system emulator design.

\subsection{Motivation for Solving the Existing Problems}

The U.S. government keeps searching for new ways to promote energy conservation efficiency, eliminate dependency on fossil fuel, reduce carbon emissions, 
and meet the increasing demand for energy consumption. Also, consumers in the utility grid system are looking for reliable and low cost energy supplies. At the same time, utility companies also want to improve their power system reliability, and reduce the cost of energy generation, transmission and distribution. As mentioned earlier, smart hybrid AC-DC power system with PEVs and renewable energy sources have become a popular and effective solution to address challenges such as global fossil energy scarcity, greenhouse gas emissions, global warming, utility grid over loading, high operation cost, and grid security.

Therefore, much more research about the hybrid AC-DC power systems with PEVs and renewable energy sources is needed. The research in this dissertation will cover several subjects related to hybrid AC-DC power systems modeling, design, forecasting, optimization, control, protection, and economic analysis.

\subsubsection{Essential applications are in practice}

The techniques developed in this dissertation will be verified on a hybrid AC-DC power system testbed with various functions including a real-time PEV parking garage emulator hardware platform. This gives a great opportunity to plan, model, design, and create samples for a variety of power system components that can be applied in future hybrid AC-DC power systems with different scales. This hybrid AC-DC power system has two major parts: AC and DC subsystems. The AC subsystem has local generators, AC loads and can be connected utility grid connection through a local transformer. The DC subsystem has PV emulator, DC load, and a PEV parking emulator with five lithiumion battery banks and five bidirectional DC-DC converters that act as five PEVs and their charger. A bidirectional AC-DC inverter is used to links the $\mathrm{AC}$ and the $\mathrm{DC}$ subsystems. 


\subsubsection{Hybrid power system real-time analysis and operation}

Operating a hybrid AC-DC power system with multiple components requires an infrastructure to monitor, protect and control the system in real-time. For this purpose, this research focuses on the application of modern control algorithms involving modeling, forecasting, optimization, and real-time control to a hybrid power system with renewable energy source emulator and PEVs emulator that can be verified through hardware in the loop systems. This is because the planning, construction, and managing of future hybrid power systems, especially the ones with high penetration of renewable energy sources and PEVs need those technologies studied in this dissertation. Also, realtime operation and physical infrastructure of emulated hybrid AC-DC power systems enables researchers to experimentally run scenarios and investigate hidden issues in virtual systems.

\subsubsection{Smooth PEV charging impact to utility grid}

Grid integration of large numbers of PEVs is both a challenge and opportunity to the current power grids. If not managed properly, large numbers of PEVs may degrade the power system stability and reliability. If regulated wisely, the PEVs batteries can serve as distributed energy storage to relieve the peak demand and keep the real-time balancing of generation and load. This dissertation addresses the challenges of charging impact to utility grid brought by large numbers of PEVs and the objectives are as follows:

- Reduce the charging cost based on the utility grid energy prices;

- Smart control the PEV charging process to shift peak time and reduce the peak power demand;

- Maximize the utilization of renewable energy sources to charge the PEVs; 
- Provide V2G services such as grid frequency regulation, reactive power compensation, and load peak shaving to help the utility grid improve the overall system reliability, efficiency, and stability.

\subsubsection{Hybrid power system protection}

Protection plays an important role in hybrid AC-DC power system operation and management. The future hybrid power systems should function with the protection of electrical power systems from faults through the isolation of faulted parts from the rest of the electrical network. The objective of a protection scheme is to keep the hybrid power system stable by isolating only the components that are under fault, whilst leaving as much of the network as possible still in operation. Thus, protection schemes must apply a very pragmatic and pessimistic approach to clearing system faults. This dissertation also put efforts to solve two kinds of protections: hybrid system operation with pulsed loads protection and lithium-ion battery banks SOC and thermal unbalancing protection. The hybrid system operation with pulsed loads protection will prevent the hybrid power system from over loading, over voltage, and over current which may damage the major components such as AC generators and energy storage systems. The lithium-ion battery banks SOC and thermal unbalancing protection will greatly improve the performance of the lithium-ion battery banks and extend the battery banks operation lifetime.

\subsubsection{Verification of developed techniques on hardware based Testbed}

In this dissertation, the detailed design process of hardware based PEV parking garage hybrid AC-DC power system emulator will be given. The advantages of establishing such a system setup are as follows: 
- Compare different solutions and approaches in hybrid AC-DC power system modeling, optimization, control and protection.

- Provide faster and more reliable results for Hybrid AC-DC power systems due to the availability of an actual power system model and components.

- Solving the problem in presence of real environmental conditions, such as PEV charger noise and harmonics, non-ideal conditions as well as some hidden or neglected factors which may be concealed in simulation study.

- Investigate the design and operation of the high efficiency components needed to build different scales of PEV parking garage hybrid power systems.

- Investigate and limit the impacts of PEV charging to the utility AC grid.

- Develop a platform for community and commercial awareness regarding PEV charging hybrid power systems and their economic and environmental values.

- Provide a training and education environment not only for research but also for future power engineering students.

\subsection{Literature Review}

Electrification of transportation has become an important industry trend supported by the interest of energy independence. For the past few years, PEV technology development has gained immense popularity because of the PEV's ability to reduce greenhouse gases (GHGs) and its ability to alleviate the effects of rising gasoline prices on the consumer [72]. Recent analysis indicates that significant portions of the U.S. gasoline-operated vehicle fleet could be fueled with the available electric capacity. In fact, about $84 \%$ of the total energy needed to operate all of the cars, pickup trucks, and 
SUVs in the U.S. could be supported using the generation capacity currently available [73]. The National Renewable Energy Laboratory (NREL) concluded that large-scale deployment of PEVs will have limited, if any, negative impacts on the electric power generation requirements [74]. Recent studies showed that if PEVs displaced half of all vehicles on the road by the year 2050 , they would require only an $8 \%$ increase in electricity generation (4\% increase in capacity) [75]. These data have encouraged the continuing development of PEVs.

In [76] an experimental control strategy for an electric vehicle charging system composed of a photovoltaic (PV) array, converters, power grid emulator and programmable DC electronic load that represents a li-ion battery emulator is presented. The designed system can supply power to the battery at the same time as PV energy production. The applied control strategy aims to extract maximum power from PV array and manages the energy flow through the battery with respect to its state of charge and taking into account the constraints of the public grid. In [77] how a solar powered building interacts with energy storage and how it can be used to power a PEV and to support the grid with peak shaving, load shifting, and reducing annual energy usage is demonstrated. A smart micro-grid equipped with renewable sources will be popular and it will be common for the owners of the smart house to profit from utilizing their alternative energy assets and PEVs. Because of bidirectional power flow between smart houses and the utility gird, at a certain time, the power generated by the renewable sources may be larger than the power needed by the load and the power can be sold to the utility grid. Furthermore, with PEVs, the owner can choose the best time (e.g. when the price of the 
power is the highest during a day) to sell the power to the grid to get the maximum benefit.

In order to limit the impact of PEVs on the grid, different priorities and charging price should be developed. With different priority levels, PEVs in a parking lot will be charged at different periods. For a certain time, the controller should make a decision on which PEVs should be charged and which should not base on priority levels, power flow limitation, and the objective of minimizing of total cost. In [78] an intelligent method for scheduling usage of available energy storage capacity from PEVs is proposed. The batteries on these vehicles can either provide power to the grid when parked, known as V2G concept or take power from the grid to charge the batteries on the vehicles. A scalable parking lot model is developed with different parameters assigned to fleets of vehicles. Optimal solutions that maximize profits to vehicle owners while satisfying system and vehicle owners' constraints is found by using binary particle swarm optimization algorithm. The system should update the charging state every several minutes, because of the stochastic characters of the number of PEVs connected to the grid and the energy needed by them. The updating can be accomplished by using the gossip based message dissemination schemes [79].

As mentioned, during V2G operations, a big PEVs parking station can provide many grid service such as reactive power support. In [80], the potential of a plug-in electric vehicle parking lot (Smart-Park) for energy storage in a power system with a large wind farm has been investigated. Also, a fuzzy logic based coordination controller of the wind farm and the distributed Smart-Parks has been proposed in this paper. The fuzzy controller uses the total state of charge of the Smart-Parks and the difference 
between instantaneous demand and the available wind power generation as the inputs and thereby generates the charging or discharging power commands of the Smart-Parks and the pitch angle reference for the wind turbine. In [81], the potential of a low-cost solution that utilizes the reactive power and voltage support capabilities of plug-in vehicles parked in charging stations (Smart-Parks) so that they can behave as virtual STATCOMs is explored. In [82], an idea is presented for utilizing PEV parking lots (Smart-Parks) to reduce the shock on the transmission lines connected to a wind farm caused by drastic variations in wind-power generation and to prevent the neighboring lines from overloading.

By using a properly designed PEV load dispatch algorithm, the load power consumption curve can be shaped much more manageable. At that time, the control of the power generated by the generator based on future load forecasting will be much easier. If the daily power consumption is a constant or changing smoothly, more base energy sources can be used which are much cheaper. In [83], a smart load management control strategy for coordinating PEV charging based on peak demand shaving, improving voltage profile, and minimizing power losses is proposed. Furthermore, the developed smart load management approach takes into consideration the PEV owner's preferred charging time zones based on a priority selection scheme. The impact of PEV charging stations and typical daily residential loading patterns are also considered. In [84] a realtime smart load management (RT-SLM) control strategy is proposed and developed for the coordination of PEV charging based on real-time (e.g. every $5 \mathrm{~min}$ ) minimization of total cost of generating the energy plus the associated grid energy losses. The approach reduces generation cost by incorporating time-varying market energy prices and PEV 
owner preferred charging time zones based on priority selection. The RT-SLM algorithm appropriately considers random plug-in of PEVs and utilizes the maximum sensitivities selection (MSS) optimization.

At the same time, batteries have emerged as the most prominent energy storage device and are rapidly being integrated into smart hybrid power systems and PEVs. Battery systems serve to enhance the performance of smart grids utilizing renewable energy sources, specifically those in islanding configurations. These battery systems should compensate for the intermittent nature of renewable sources and serve to regulate frequency and supply power to increase the reliability and efficiency of a power system. Lithium-ion batteries in particular are the most popular choice and are likely to remain as the ideal battery chemistry [85]. They're valuable not just for the great amount of energy they can store in comparatively little space and at a lighter weight but also for the big spike of current they can kick out. The recent Boeing 787 Dreamliner lithium-ion battery faults in Japan and Boston have illustrated the importance of developing reliable and efficient battery management systems in high energy density battery systems [86]. PEV's have also seen an expansive growth over the past several years and with their growth in popularity increases the demand for more reliable and efficient battery management systems. PEVs rely heavily on the capability of their battery management systems to monitor and manage the state of charge and temperature variations in order to increase the capacity, reliability, and lifetime of the individual cells that make up their battery system. Imbalances and variations between individual cells often occur throughout the battery banks and these variations can range from discharge rates and internal impedances to energy densities. Imbalances in cell temperatures and state of charge can 
lead to individual cells being overcharged, over discharged, and overheated. These failures can propagate throughout the entire battery system and severely diminish the efficiency and reliability of the system. The optimal operating environment of lithium-ion battery ranges from 30 to $40^{\circ} \mathrm{C}$ and every $10{ }^{\circ} \mathrm{C}$ increase in the temperature halves the life of the battery cell [87]. The specific characteristics and needs of the hybrid power system and PEVs, such as deep charge/discharge protection and accurate SOC and state-ofhealth estimation, intensify the need for a more efficient battery management system.

In [88], T. Huria et al presented a high fidelity electrical model with thermal dependence for characterization and simulation of high power lithium battery cells. This work gives a method to model lithium ion batteries with the pulse charging and discharging performance characters. The model is able to estimate the SOC and temperature of single lithium-ion batteries. In [89], Kim, T et al gave a multi-cell battery bank system design method for electric and plug-in hybrid electric vehicles, which has the function of SOC self-balancing. The battery banks performance is also dependent on the temperature, an overheated single cell may ruin the whole system. Therefore, more research is needed to design the energy storage management and protection system to take both SOC and thermal imbalance issues into consideration. In [90], Lu, X. et al presented the coordinated control of distributed energy storage systems in dc micro grids. In order to balance the SOC of each energy storage unit, an SOC-based adaptive droop control method is proposed. In this decentralized control method, the droop coefficient is inversely proportional to the nth order of SOC. By using a SOC-based droop method, the energy storage units with higher SOC deliver more power, whereas the ones with lower SOC deliver less power. Therefore, the energy stored in the ESU with higher SOC 
decreases faster than that with lower SOC. The SOC difference between each energy storage unit gradually becomes smaller, and finally, the load power is equally shared between the distributed energy storage units.

\subsection{Problem Statement}

With the high penetration of PEVs and renewable energy sources in the future, the existing U.S. utility grid may not have sufficient capability to handle with high power flow. Therefore, novel energy management methods together with high efficiency power system architectures are needed to keep the future utility grid operating in stable mode. This dissertation focuses on designing a hybrid AC-DC power system with high penetration of renewable energy sources and PEVs. The research problem has the following eight main facets:

- To maximize the utilization of renewable energy for smart parking systems with PEVs, renewable energy sources, and AC utility grid.

- To investigate the power quality aspects related to the hybrid AC-DC power system under study with PEV parking systems and renewable energy sources.

- To study, model, simulate, implement and control the various power electronic converters involved in a hybrid AC-DC power system such as the bidirectional DC-DC converters for PEVs, DC-DC boost converter with maximum power point tracking (MPPT) function for photovoltaic panel and so on.

- To find the optimal solutions for planning and construction of future PEV hybrid parking garage power system with different scales. 
- To minimize power losses, improve voltage profile and limit the impact of PEVs to the hybrid AC-DC power system under study.

- To regulate voltage and frequency of the hybrid power system AC side and maintain the DC side voltage stability by using active and reactive power compensation through $\mathrm{V} 2 \mathrm{G}$ and $\mathrm{V} 2 \mathrm{H}$ functions.

- To enhance the hybrid power system reliability by introducing effective protection solutions to lithium-ion battery banks, power electronic devices, and securing the communications in the system.

- To develop real-time power management algorithms in order to smartly operate hybrid AC-DC systems. These algorithms will be focused on solving several power system issues such as optimal operation of hybrid renewable energy systems or smart distribution of limited energy to different PEVs.

\subsection{Purpose of Research}

The aim of this research is to design and verify smart charging strategies for different scales of PEV parking garage hybrid power systems with high penetration of renewable energy sources, and discuss their intelligent operation and protection. The proposed smart charging hybrid power system has the capability to regulate and spin the reserve, reshape the load curve, serve as external storage for renewable sources, and generate revenue by buying and selling power at different times according to variable price curves. At the same time, the hybrid power system can monitor the PEVs in it and smartly distribute the energy among them. Moreover, control of the charging process of the PEVs with the proposed intelligent energy management algorithms will help the 
hybrid power system improve the power quality and boost the renewable energy harvesting.

All of this will accelerate the renewable energy harvesting and increase the amount of PEVs in the future market, which in the end will decrease greenhouse gas emissions, slow down global warming, and reduce the utility grid dependence on fossil energy.

\subsection{Original Contribution of This Dissertation}

The conventional aging U.S AC utility power system needs significant changes and improvements in the energy generation stage, transmission stage and distribution stage to address the challenges that will be brought by high penetration of renewable energy sources, distributed generations and critical electronic loads such as PEVs. Therefore, new power system architectures together with effective power and energy management algorithm need to be designed to enhance the modern power systems' reliability, stability, efficiency and security in both AC and DC sides.

This dissertation introduced a hybrid AC-DC power system with PEVs and renewable energy sources. This hybrid AC-DC power system has the capability to be operated in both grid-connected and islanding modes in real-time manner. Artificial intelligent tools such as ANN, GA and FLC are used in design, optimization and control of the hybrid power system. Throughout this dissertation, novel ideas and concepts are presented on different scales, from the component level to the system scale.

On the component scale, the performance of the current state-of-the-art DC-DC converters used to integrate renewable energy sources and connect PEVs to the DC bus is 
depicted. Moreover, a novel wireless control of bidirectional DC-DC converter power flow for battery charging/discharging management is proposed. Also, the active and reactive power decoupled control for bidirectional AC-DC inverter is simulated and implemented in hardware. Furthermore, a novel FLC based battery banks management system with SOC and thermal self-balancing functions is developed and implemented to control lithium-ion battery banks in PEVs and hybrid power systems.

On the system scale, novel scale optimization method is given for planning and construction of renewable energy farms and energy storage systems to smoothly supply large amount of energy to the utility grid with limited impacts. Also, FLC based charging control of PEVs in different scales of PEV parking garages are given with the consideration of several factors such as: limit charging impact to utility grid, maximize the utilization of renewable energy, minimize the charging cost, and support the utility grid through the V2G services. Moreover, the detailed analysis of the operation of each component in the hybrid AC-DC power system in system point of view are given in both grid-connected and islanding modes.

\subsection{Dissertation Organization}

This dissertation is prepared in eleven chapters including the current chapter and they are organized as follows:

Chapter 1 presents a background about the hybrid AC-DC power system, including the overviews of the benefits and current challenges about the hybrid power system with high penetration of renewable energy sources and PEVs, the role of lithiumion batteries as energy storage in hybrid power systems and PEVs, and the application of 
artificial intelligence to hybrid power systems. It also includes the literature review, problem statement, and purpose of this research, originality contribution of this research and the outline of this dissertation.

Chapter 2 introduces a detailed hybrid AC-DC power system operation with PEVs. It analyzes the potential PEV charging impact to the utility grid while explaining PEVs charging control through $\mathrm{V} 2 \mathrm{~V}$ and $\mathrm{V} 2 \mathrm{G}$ services. It also gives the communication requirements for hybrid AC-DC power systems to fulfill those functions.

Chapter 3 introduces the way of utilizing renewable energy sources in hybrid power systems, from renewable energy pattern analysis to maximizing renewable energy sources with power electronics devices and MPPT technologies. It also contains analysis of the renewable energy sources potential impact to the hybrid power system.

Chapter 4 gives a novel optimization method for sizing a renewable energy farm consisting of batteries and ultra-capacitors in a hybrid power system. The proposed sizing method fully utilizes the energy generated from the renewable energy farm and limits power fluctuation within the utility grid, which will greatly improve grid stability and reduce construction and maintenance costs. A two-step optimization process appropriately sizes the renewable energy farm and the energy storage system by using a genetic algorithm. A case study of a hybrid power system located in Key West, Florida is presented to verify the advantages of the proposed optimal sizing method.

Chapter 5 presents a battery management system that is able to self-heal from faults in order to avoid SOC and thermal imbalances using cell switching circuits. An accurate lithium-ion battery thermal model is presented to emulate the lithium-ion battery state during the charging and discharging process. A novel fuzzy logic based battery bank 
management system is presented. The battery bank management system has self-healing capabilities to protect itself from SOC and thermal imbalances.

Chapter 6 proposes a FLC based smart charging algorithm for a small scale hybrid AC-DC PEV workspace parking garage charging system. A model of a $318 \mathrm{~V}$ grid-connected DC power distribution network combined with PV and PEV parking garage is designed and simulated. Accurate PV and PEV power stochastic models based on statistical theory are studied.

Chapter 7 introduces the economic analysis of a real-time large scale PEV network power flow control algorithm for charging impact limitation and V2G services. An optimization process for renewable energy farm with both solar and wind energy by using genetic algorithm (GA) is given. The studies include both the feasibility and economic analysis.

Chapter 8 proposes the distributed power control of major components in the hybrid AC-DC power system with renewable energy source, energy storage and pulsed loads. A bidirectional fully controlled AC-DC inverter is used to link the AC subsystem with the DC subsystem. A DC-DC boost converter with a MPPT function is implemented to maximize the energy generation from the PV farm. Bidirectional DC-DC converter is applied to connect a lithium-ion battery bank to the DC subsystem. The detailed power control strategies of different power electronic devices for the hybrid micro grid operated in grid-connected mode and islanding mode are proposed and analyzed. Simulation and hardware experiments are done with the mitigation of pulsed loads. 
Chapter 9 presents of the methodology of using Micro controllers and wireless transmitters to monitor and control the charging/discharging of lithium-ion battery banks in the DC subsystem of a hybrid AC-DC power system.

Chapter 10 gives the description of a laboratory scaled PEV car park infrastructure emulator smart hybrid power system testbed developed at the Energy Systems Research Laboratory, Florida International University. The proposed control algorithms in former chapters are tested on the testbed. Hardware experiments are done with the results showing that the proposed PEVs car park emulator can smartly charging the battery banks and help the AC micro grid regulating the system frequency through V2G services.

Chapter 11 summarizes this dissertation, concludes the significance of this research, discusses the results, and finally makes recommendations for the future research. 


\section{Hybrid AC/DC Power System with Plug-in Electric Vehicles}

\subsection{Introduction}

The debate of AC power systems versus DC power systems is historical and as old as the evolution of the first commercial power systems themselves. In the late $1880 \mathrm{~s}$, a variety of inventions across the United States and Europe led to a full-scale battle between alternating current and direct current distribution [91].

In 1886, Ganz Works, an electric company located in Budapest, electrified all of Rome with AC power. Thomas Edison, on the other hand, had constructed 121 DC power stations in the United States by 1887. A turning point in the battle came when George Westinghouse, a famous industrialist from Pittsburg, purchased Nikola Tesla's patents for AC motors and transmission the next year.

In the late $1800 \mathrm{~s}, \mathrm{DC}$ could not be easily converted to high voltages. As a result, Edison proposed a system of small, local power plants that would power individual neighborhoods or city sections. Power was distributed using three wires from the power plant: +110 volts, 0 volts, and -110 volts. Lights and motors could be connected between either the $+110 \mathrm{~V}$ or $110 \mathrm{~V}$ socket and $0 \mathrm{~V}$ (neutral). $110 \mathrm{~V}$ allowed for some voltage drop between the plant and the load (home, office, etc.). Even though the voltage drop across the power lines was accounted for, power plants needed to be located within 1 mile of the end user. This limitation made power distribution in rural areas extremely difficult, if not impossible. 
With Tesla's patents, Westinghouse worked to perfect the AC distribution system. Transformers provided an inexpensive method to step up the voltage of AC to several thousand volts and back down to usable levels. At higher voltages, the same power could be transmitted at much lower current, which meant less power lost due to resistance in the wires. As a result, large power plants could be located many miles away and service a greater number of people and buildings. Over the next few years, Edison ran a campaign to highly discourage the use of AC in the United States, which included lobbying state legislatures and spreading disinformation about AC. Edison also directed several technicians to publicly electrocute animals with $\mathrm{AC}$ in an attempt to show that $\mathrm{AC}$ was more dangerous than DC. In an attempt to display these dangers, Harold P. Brown and Arthur Kennelly, employees of Edison, designed the first electric chair for the state of New York using AC. In 1891, the International Electro-Technical Exhibition was held in Frankfurt, Germany and displayed the first long distance transmission of three-phase AC, which powered lights and motors at the exhibition. Several representatives from what would become General Electric were present and were subsequently impressed by the display. The following year, General Electric formed and began to invest in $\mathrm{AC}$ technology. Westinghouse won a contract in 1893 to build a hydroelectric dam to harness the power of Niagara Falls and transmit AC to Buffalo, NY. The project was completed on November 16, 1896 and AC power began to power industries in Buffalo. This milestone marked the decline of DC in the United States. While Europe would adopt an AC standard of 220-240 volts at $50 \mathrm{~Hz}$, the standard in North America would become 120 volts at $60 \mathrm{~Hz}$. 
Swiss engineer René Thury used a series of motor-generators to create a highvoltage DC (HVDC) system in the 1880s, which could be used to transmit DC power over long distances. However, due to the high cost and maintenance of the Thury systems, HVDC was not adopted for almost a century. With the invention of semiconductor electronics in the 1970s, economically transforming between AC and DC became possible. Specialized equipment could be used to generate high voltage DC power (some reaching $800 \mathrm{kV}$ ). Parts of Europe have begun to employ HVDC lines to electrically connect various countries. HVDC lines experience less loss than equivalent AC lines over extremely long distances. Additionally, HVDC allows different AC systems (e.g. 50 $\mathrm{Hz}$ and $60 \mathrm{~Hz}$ ) to be connected. Despite its advantages, HVDC systems are more costly and less reliable than the common $\mathrm{AC}$ systems. In the end, Edison, Tesla, and Westinghouse may all have their wishes come true. AC and DC can coexist and each serves a purpose.

Nowadays, due to environmental issues caused by fossil fueled energy sources and the exhaustion of the global fossil energy reserves, more renewable energy systems such as solar farms and wind farms are connected in utility power systems. The majority of renewable energy sources generate DC power, or need a DC link to connect to the utility grid and as a result of increasing modern DC loads such as PEVs and light-emitting diodes (LEDs), DC micro grids have begun to emerge for their benefits in terms of efficiency, cost, and being able to eliminate the DC-AC or AC-DC power conversion stages and their accompanied energy losses.

On the other hand, the majority of the utility power grids are still in AC type, including energy generation, transmission to distribution. Purely DC power systems are 
not expected to emerge exclusively in the power system. Therefore, the design and management of hybrid AC-DC power systems which link AC power system with DC power subsystem and employ the benefits of both systems, has become an interestingly popular area of research.

PEVs are gaining popularity due to several reasons; they are convenient, visually appealing, quiet, and produce less pollution in the environment. However, the increasing number of PEVs can have a huge impact on the utility grid or small scale hybrid AC-DC power system if properly designed smart charging techniques are not utilized. Uncoordinated and random charging activities could greatly stress the distribution system, causing several kinds of technical and economic issues such as suboptimal generation dispatches, huge voltage fluctuations, degraded system efficiency and economy, as well as increasing the likelihood of blackouts due to network overloads. On the other hand, if properly managed with fulfillment of the $\mathrm{V} 2 \mathrm{G}$ and $\mathrm{V} 2 \mathrm{~V}$ functions, PEV batteries can serve as distributed energy storages to relieve peak demand and keep the real-time balancing of generation and load in the hybrid power system.

This chapter is organized as follows: Section 2.2 gives the introduction of the state of the art of hybrid AC-DC power systems and their challenges and communication requirements in both grid-connected and islanding operation modes. Section 2.3 analyzes the present market available PEV charging infrastructures and their possible charging impacts to the utility grid at transmission and distribution levels. Section 2.4 describes the vehicle to grid technology together with its challenges and future market. Section 2.5 presents the concept of vehicle to vehicle technology and its challenges and future possible markets. Finally, a conclusion is given in Section 2.6. 


\subsection{Hybrid AC-DC Power System Architectures}

Hybrid AC-DC power systems by definition contain a number of power generation devices such as wind turbines, photovoltaics, micro-hydro and/or fossil fuel generators, and range from small systems designed for one or several homes to very large ones for remote island grids or large communities. The idea is to combine the AC and DC subsystems through a bidirectional AC-DC inverter and establishing a hybrid AC-DC smart grid in which AC or DC energy sources and loads can flexibly integrate into the hybrid systems and power can smoothly flow between the two subsystems. The conceptual hybrid AC-DC power system configuration is shown in figure 2.1. The hybrid AC-DC power systems may have various AC and DC loads and power sources such as wind turbines, photovoltaic panels, flywheel, fuel cell and local generators connected to the AC and DC subsystems respectively. The AC and DC buses are linked together through three phase bidirectional AC-DC inverter. The AC bus of the hybrid power system can be tied to the utility grid.

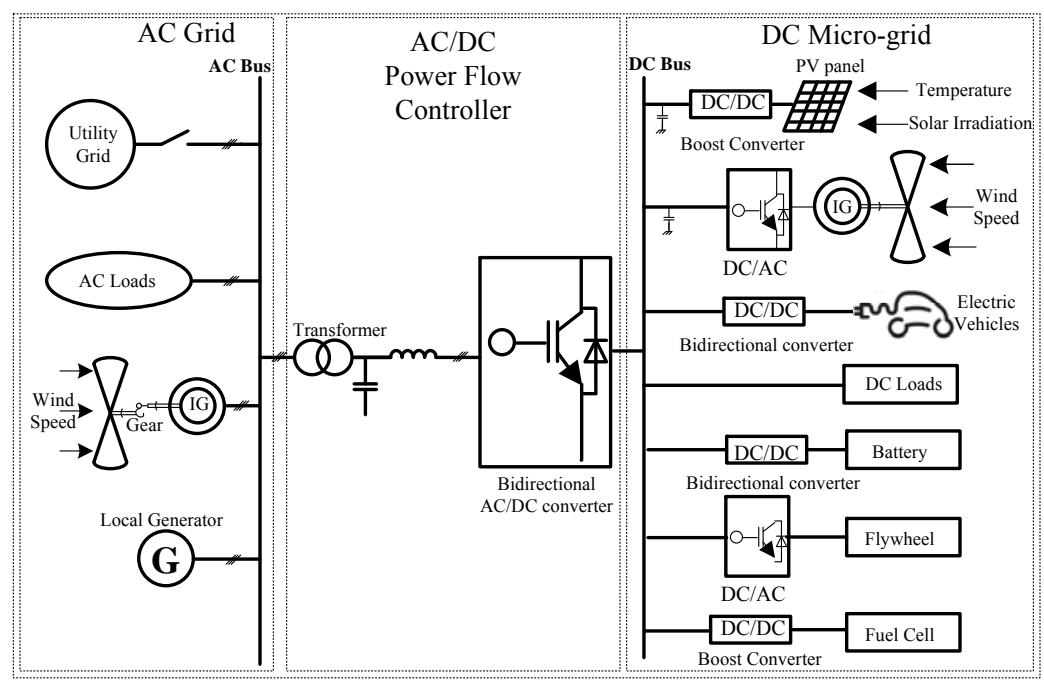

Figure 2.1 Hybrid AC-DC power system configuration 


\subsubsection{Grid Connected Mode and Islanding Mode}

Like other kinds of microgrids, the hybrid AC-DC power system can be operated in either grid connected or islanding modes and the control system should be able to support the two operating modes as well as transition between these modes.

In grid connected mode, due to connecting to the main utility grid, the load demand can be met all the time, and the $\mathrm{AC}$ bus is strong enough that the voltage amplitude and frequency don't need to be regulated. Therefore, the main operation objective of the hybrid AC-DC power system is to maximize the utilization of renewable energy sources and generate more revenues according to the energy market price. The bidirectional AC-DC inverter is to regulate DC bus voltage and reactive power flow. The power electronics devices connected to the renewable energy sources are controlled to provide the maximum power. When the power generated form the DC energy sources is greater than the load consumption from the DC bus, the bidirectional AC-DC inverter can transfer energy to the AC side. When the total power generated is more than the load in the hybrid power system, the extra energy will be injected to the utility grid. Otherwise, the hybrid AC-DC power system will receive power from the utility grid. In grid connected mode, the energy storage devices such as battery banks are not essential because the power flow is balanced in the hybrid power system by the utility grid. However, those energy storage devices can help the hybrid power system increase the operating revenues by intelligently buying and selling power to the utility grid. For example, when the energy price is low, the hybrid power system can store extra energy in the batteries instead of selling to the utility grid. Further, with precise renewable energy source output and local load fasting, hybrid power systems can purchase cheap energy in 
advance from utility grid and store it in the energy storage devices for an upcoming local load peak.

When the hybrid AC-DC power system is operating in islanding mode, the power flow can't be balanced by the utility grid. Also, the AC bus voltage is not as robust as it is in grid-connected mode. Therefore, the highest priority is to keep the whole system stable and maintain a reliable power supply to the loads. The control objective of the hybrid AC-DC power system operated in islanding mode is to maximize the satisfaction rate of the load with minimum operational cost, which can be accomplished by smart control of various power electronic devices. In islanding mode, the energy storage devices play a significant role for both power balance and voltage stability. DC bus voltage is regulated to be stable by the battery together with the bidirectional DC-DC converter. The bidirectional AC-DC inverter is used to provide a high quality AC bus voltage with stable amplitude and frequency. The renewable energy sources can be operated on maximum power point tracking or off maximum power point tracking based on the SOC of the energy storage devices and power flow situations in the hybrid power system.

\subsubsection{Hybrid AC-DC power system challenges}

The objective of energy management of hybrid AC-DC power systems is to keep the whole system stable while minimizing the system's operating expenses such as fuel costs, maintenance costs, and purchasing costs of electricity from the utility grid. Multiple power electronic devices are required to cooperate together and multiple reverse conversions are required in individual AC or DC subsystems. This may add additional losses to the system operation and will make the hybrid power system more complicated. Therefore, there are still several challenges for the hybrid AC-DC power system to 
provide reliable, high quality electric power to loads in an environmentally friendly and sustainable way. The possible challenges for future hybrid AC-DC power systems are:

- How to integrate and combine different types of energy sources that have unique operating constraints and benefits.

- How to improve the quality issues such as frequency, voltage, harmonics and system stability.

- How to optimize the scales and locations of each component in the hybrid system and limit its impact to the utility grid.

- How to determine when an islanded hybrid power system should be formed in the face of the array of abnormal conditions that the utility can experience.

- How to provide segments of the hybrid AC-DC power system with sufficient coordinated fault protection while operating in islanding mode.

Therefore, future hybrid AC-DC power systems should have an advanced structure and control scheme that can facilitate the connections of various AC and DC generation systems, energy storage devices, and various critical AC and DC loads with the optimal asset utilization and operational efficiency.

\subsubsection{Hybrid AC/DC power system communication requirements}

One of the important technological areas of the hybrid AC-DC power system is integrated two-way communications, which allows for dynamic monitoring of electricity use as well as the potential for automated electricity use scheduling.

The potential promises of the Smart Grid are numerous, including: (1) improved reliability; (2) increased physical, operational, and cyber security, as well as resilience 
against attack or natural disasters; (3) ease of repair, particularly remote repair; (4) increased information available to consumers regarding their energy use; (5) increased energy efficiency along with the environmental benefits gained by such efficiency; (6) the integration of a greater percentage of renewable energy sources, which can be inherently unpredictable in nature; (7) the integration of plug-in electric vehicles; and, (8) a reduction in peak demand [92].

Therefore, effective communication network infrastructure is one of the key factors for hybrid AC-DC power systems with high penetration of PEVs and renewable energy sources to operate with high power quality, reliability, efficiency, security and low cost.

\subsection{Plug-in Electric Vehicles Charging Impact to Utility Grid}

The battery capacity of a fully charged PEV from electric vehicle automakers (such as Nissan) is about $20 \mathrm{kWh}$, providing it with an electrical autonomy of about 100 miles. Tesla Motors released their Model S with battery capacities of $60 \mathrm{kWh}$ and $85 \mathrm{kWh}$ with the latter having an estimated range of approximately $480 \mathrm{~km}$. PEVs can be charged slowly or quickly, at home or at a public charging station. The charging infrastructures are important components necessary to accommodate and support the successful rollout of PEVs. Charging equipment for PEVs is classified by the rate at which the batteries are charged. Charging times vary based on how depleted the battery is, how much energy it holds, the type of battery, and the type of charger. The charging time can range from 15 minutes to 20 hours or more, depending on these factors. Three main types of charging levels are commonly used for PEVs in U.S., the detail is shown in Table 2.1. 
Table 2.1 U.S. Standard Plug-in Electric Vehicle Charging Levels

\begin{tabular}{|c|c|}
\hline Charging Type & Characteristics \\
\hline Level 1 & $\begin{array}{l}\text { - } 110 / 120 \mathrm{~V}, \mathrm{AC}, 15-20 \mathrm{amp} \\
\text { - Does not require installation and can use standard } 110 \mathrm{~V} \text { electrical } \\
\text { outlet } \\
\text { - Typical charge time: } 8-12 \text { hours }\end{array}$ \\
\hline Level 2 & $\begin{array}{l}\text { - } 208-240 \mathrm{~V}, \mathrm{AC}, 15-30 \mathrm{amp} \\
\text { - } \quad \text { Requires special installation with average cost of } \$ 1,000 \\
\text { - } \quad \text { Typical charge time: } 3-8 \text { hours }\end{array}$ \\
\hline Level 3 & $\begin{array}{l}\text { - } \text { DC fast charging stations } \\
\text { - } 480 \text { to } 600 \mathrm{~V}, \mathrm{DC}, 125 \mathrm{amp}, 55 \mathrm{~kW} \text { or higher } \\
\text { - } \quad \text { Requires special installation } \\
\text { - } 80 \% \text { charge in } 30 \text { minutes }\end{array}$ \\
\hline
\end{tabular}

Without proper, management, charging of PEVs may cause a huge impact to the utility grid, increasing the load peak demand and may causing grid instability. The level 3 charging stations can absorb $55 \mathrm{~kW}$ or higher power from the utility grid to charge a single PEV. This power consumption is several times more than the average U.S. household power consumption. Therefore, if several PEVs are charging at the same time in the a level 3 DC charging station, the charging behavior will absolutely generate a pulsed load effect to the utility grid, which brings impacts to the grid power transmission system such as transmission line over current issues and transformer degradation issues. Even for level 1 and 2 charging terminals, big communities or PEV parking garages with a large amount of PEVs charging uncontrolled may cause the DC subsystem voltage to drop and AC subsystem frequency variations. Therefore, the PEVs charging impact to the utility grid transmission and distribution systems must be limited. 


\subsection{Vehicle-to-Grid Technology}

As mentioned in Chapter 1, if smartly regulated, the PEVs can serve as distributed energy storage devices when they are parked in the garage or community for a long period of time. $\mathrm{V} 2 \mathrm{G}$ services can help the local grid relieve the peak demand and maintain the real-time balancing of power.

\subsubsection{Vehicle to grid services}

V2G is the most essential technology for PEV adoption in future hybrid AC-DC power systems. PEVs can be viewed as distributed energy storage and can act as both controllable loads and energy sources with V2G. There are several ways in which the energy stored in PEVs can be used including: peak shaving, local load smoothing, backup power supply, smoothing output of intermittent generation, and ancillary services such as voltage regulation, frequency regulation, etc. Considering the fact the PEVs are utilized on average for less than $5 \%$ of the day, being compensated for the rest of time while being charged gives a considerable benefit and motivation for PEV owners to enroll their PEVs in V2G services.

There are several benefits to using PEVs as distributed energy storage in hybrid AC-DC power systems. For example, PEVs are typically located near local load and distributed energy generation, where they can conveniently be integrated into load and generation management systems. This gives the hybrid AC-DC power system the ability make the energy management decisions locally without needing to confer with the rest of the systems or other remote loads and resources. The PEVs and distributed generations are right there with the local load, and these components together can be controlled to meet local objectives, avoiding the need to rely on large generating units in the utility 
grid, which will greatly limit the PEVs charging impacts. Furthermore, some power and energy services are better served locally, making them a perfect fit for PEV V2G services. For instance, voltage control through voltage amplitude regulation support is best supplied locally, and PEVs together with their chargers can offer local reactive power compensation. Although real power can be supplied remotely, utility grid transmission and distribution losses may be as high as $7-10 \%$. Depending on the energy consumption and time of day, using PEVs can effectively decrease congestion on transmission lines and provide transmission loading relief. All these factors offer utility grids the opportunity to defer capital investment in generation and transmission upgrades while increasing the asset utilization.

\subsubsection{Vehicle to grid challenges}

Although hybrid AC-DC power systems with V2G services will have many benefits, there are still some obstacles that might delay their implementation and development in short term. The present major challenges to the V2G services are:

- How to design an effective and reliable two-way communication for the power flow management of the PEVs in the hybrid power system. To keep the hybrid power system stable and balanced, the system should have a central aggregator that can forecast and monitor the states of all the components and smartly dispatch the load.

- How to design high efficiency power electronic converters that can transfer energy between the PEVs and the utility with minimum loss.

- How to limit PEV charging impacts to the utility grid. To effectively fulfill the V2G services, a large number of PEVs are needed to be involved. However, 
for high penetration of PEVs, level 2 and 3 charging can quickly overload local distribution equipment, increase distribution transformer losses, voltage deviations, harmonic distortion, and peak demand.

- How to limit the V2G services impact to the PEV lithium-ion battery bank which may reduce the battery life. A deeper discharge increases the cell deterioration rate, resulting in a faster equivalent series resistance increase [93]. At present, a lithium-ion battery investment of $\$ 300$ per $\mathrm{kWh}$, with a

lifetime of 3,000 cycles at an $80 \%$ discharge depth suggests a battery degradation cost of $\$ 130$ per $\mathrm{kWh}[94]$.

- How to develop a common set of regulations, standards, and building codes that would be applied to widespread use of V2G services.

\subsubsection{Vehicle to grid future market}

Even though there are some challenges facing V2G services, the global V2G market still has broad development prospects. A new edition of Zpryme's Smart Grid Insights (sponsored by ZigBee Alliance), features the V2G future market [95]. This 73page report is based on extensive research on market data and forecasting models in the automotive and utility sectors, as well as government targets and programs. The report estimates that by 2020 there will be over one million V2G vehicles on the road, with an estimated market value of $\$ 26.6$ billion. In addition, they forecast a $\$ 6.7$ billion infrastructure market, and a $\$ 10.5$ billion technology market, for a total market of $\$ 43.8$ billion. That large a market is going to create a lot of jobs. The US portion of the market is estimated at $\$ 12.7$ billion. Annual revenue are expected to be $\$ 2.9$ billion. Therefore, 
future PEV V2G services have immense potentials and more research in this area is needed.

\subsection{Vehicle-to-vehicle Technology}

Vehicle to vehicle (V2V) services allows PEVs to transfer energy with each other by bidirectional charging through a local grid such as a community hybrid power system or a PEV parking garage.

\subsubsection{Vehicle to vehicle services}

When a number of PEVs are parked in a car park, the car park power system becomes a suitable micro grid for $\mathrm{V} 2 \mathrm{~V}$ operation. By using the $\mathrm{V} 2 \mathrm{~V}$ function, power reserves can be kept within the PEV car park, which can greatly reduce the power loss and trading loss between the local hybrid power system and the power grid. All these PEVs can interact with each other through the central aggregator. Specifically, the central aggregator is a control device which is able to collect all information about the PEVs and hybrid power system status, and execute the V2V function. Namely, the aggregator is responsible for coordinated control of grouping PEVs for charging and/or discharging. Thus, the PEVs in the car park can first distribute the energy amongst themselves and then interact with the grid for the overall energy request. Because of this, V2V can cooperated with renewable energy sources for the hybrid power system operation. Meanwhile, V2V can improve the development of the future smart grids.

\subsubsection{Vehicle to vehicle challenges}

Like V2G, there are still several challenges that V2V needs to overcome. The most critical issue is how to make a reliable $\mathrm{V} 2 \mathrm{~V}$ that will greatly reduce charging cost 
without influencing the driving behavior of PEV owners. The PEV drivers should be put at the highest priority level, because the drivers are unlikely to enroll their PEVs in V2V services if their PEV's SOC is low at their next departure time. Therefore, there should be a method for setting or estimating their next departure time. Further, to reduce the charging cost through $\mathrm{V} 2 \mathrm{~V}$, the forecasting technology should precisely estimate the next period's output power of renewable energy sources, the local load and the energy market price. The car park or community hybrid power system will require accurate estimates of energy generation and consumption patterns for the next few hours to smartly distribute the energy among the PEVs.

\subsection{Summary}

Hybrid AC-DC power systems that can be operated with multiple energy sources in both grid-connected and islanding modes are going to be one of the main architectures of future power systems. Hybrid AC-DC power systems integrating with renewable energy sources and PEVs will be an effective solution for reduction of whole system loss and greenhouse emissions. But there are still some challenges for hybrid AC-DC power system operation, especially the systems operated in islanding mode with a high penetration of renewable energy sources and PEVs. Despite the challenges, with smart control of the charging process, PEV charging impacts can be greatly limited. Further, with the correct fulfillment of V2G and V2V functions, PEVs can play the role of mobile energy storage devices that can support the utility grid with some ancillary services. In this chapter, the introduction of hybrid AC-DC power system architecture is given. Together with this, the operations of hybrid AC-DC power system grid-connected and 
islanding modes are described with the challenges and system communication requirements. After that, the PEV charging impacts to utility grid are analyzed. To limit those impacts, two possible solutions, $\mathrm{V} 2 \mathrm{G}$ and $\mathrm{V} 2 \mathrm{~V}$, are given with the description of present challenges and future markets. 


\section{Integration of Renewable Energy Sources into Hybrid Power Systems}

\subsection{Introduction}

Integration of renewable energy into hybrid power systems is becoming popular for modern power generation applications due to the advances in renewable energy technologies as well as the rise in prices of petroleum products. There is no shortage of renewable energy because it can be taken from the sun, wind, water, plants, and garbage to produce electricity and fuels. The combination of two or more renewable energy sources together with traditional fossil energy generation are commonly used to supply reliable energy to the power system to increase the system overall efficiency as well as balance the energy supply.

A good example of a hybrid energy system is a photovoltaic array coupled with a wind turbine. This would create more output from the wind turbine during the winter and night time, whereas during the summer and day time, the solar panels would produce their peak output. Hybrid energy systems often yield greater economic and environmental returns than wind, solar, geothermal or stand-alone systems by themselves [96].

To maximize the utilization of power generated from renewable energy resources in a hybrid power system, power electronics devices together with some control strategies are needed. For PV systems, maximum power point tracking (MPPT) is a technique that grid connected inverters, solar battery chargers, and similar devices use to get the maximum possible power from one or more photovoltaic devices, typically solar panels, though optical power transmission systems can benefit from similar technology. Solar 
cells have a complex relationship between solar irradiation, temperature, and total resistance that produces a non-linear output efficiency which can be analyzed based on the I-V curve. It is the purpose of the MPPT system to sample the output of the cells and apply the proper resistance (load) to obtain maximum power for any given environmental conditions. MPPT devices are typically integrated into an electric power converter system that provides voltage or current conversion, filtering, and regulation for driving various loads, including power grids, batteries, or motors [97].

For wind generation systems, the effective power extracted from wind is derived from the airflow speed just reaching the turbine and the velocity just leaving it. Only part of the full energy available from the wind can be extracted for energy generation, quantified by the power coefficient, $C_{p}$. The power coefficient is the relationship of the power extraction possible to the total amount of power contained in the wind. Because of the variation in wind speed, the power generated from a wind turbine may not have a constant frequency and therefore can't be directly injected to the utility grid. Consequently, power electronics devices like back-to-back converters are utilized. Also, to extract the maximum power from the wind energy, turbine blades should change their speed as the wind speed changes.

This chapter presents an overview of the integration of solar and wind energy sources into hybrid power systems by using a DC-DC converter and back-to-back converter. This chapter is organized as follows. The analysis of characteristics of solar and wind energy sources is introduced in section 3.2. Solar energy harvesting from a PV panel by using a DC-DC boost converter with maximum power point tracking, together with the detailed simulation models and hardware descriptions of PV panels and DC-DC 
boost converters are given in section 3.3. In section 3.4, a wind energy harvesting example with the basic doubly-fed induction wind turbine generator and back-to-back converter is presented. Renewable energy sources impact to a hybrid power system is presented in section 3.5. Finally, a conclusion is given in Section 3.6

\subsection{Renewable Energy Sources Characteristics Analysis}

\subsubsection{South Florida solar energy source analysis}

Solar energy, radiant light and heat from the sun, has been harnessed by humans since ancient times using a range of ever-evolving technologies. Solar radiation, together with secondary solar-powered resources such as solar heating, solar photovoltaics, solar thermal energy, solar architecture and artificial photosynthesis account for most of the available renewable energy on earth. It is an important source of renewable energy and its technologies are broadly characterized as either passive solar or active solar depending on the way they capture and distribute solar energy or convert it into solar power. Active solar techniques include the use of photovoltaic systems, concentrated solar power, and solar water heating to harness the energy. Passive solar techniques include orienting a building to the Sun, selecting materials with favorable thermal mass or light dispersing properties, and designing spaces that naturally circulate air [98]. Only a minuscule fraction of the available solar energy is used. The development of affordable, inexhaustible and clean solar energy technologies will have huge longer-term benefits. It will increase countries' energy security through reliance on an indigenous, inexhaustible and mostly import-independent resource, enhance sustainability, reduce pollution, lower the costs of mitigating global warming, and keep fossil fuel prices lower than otherwise. 


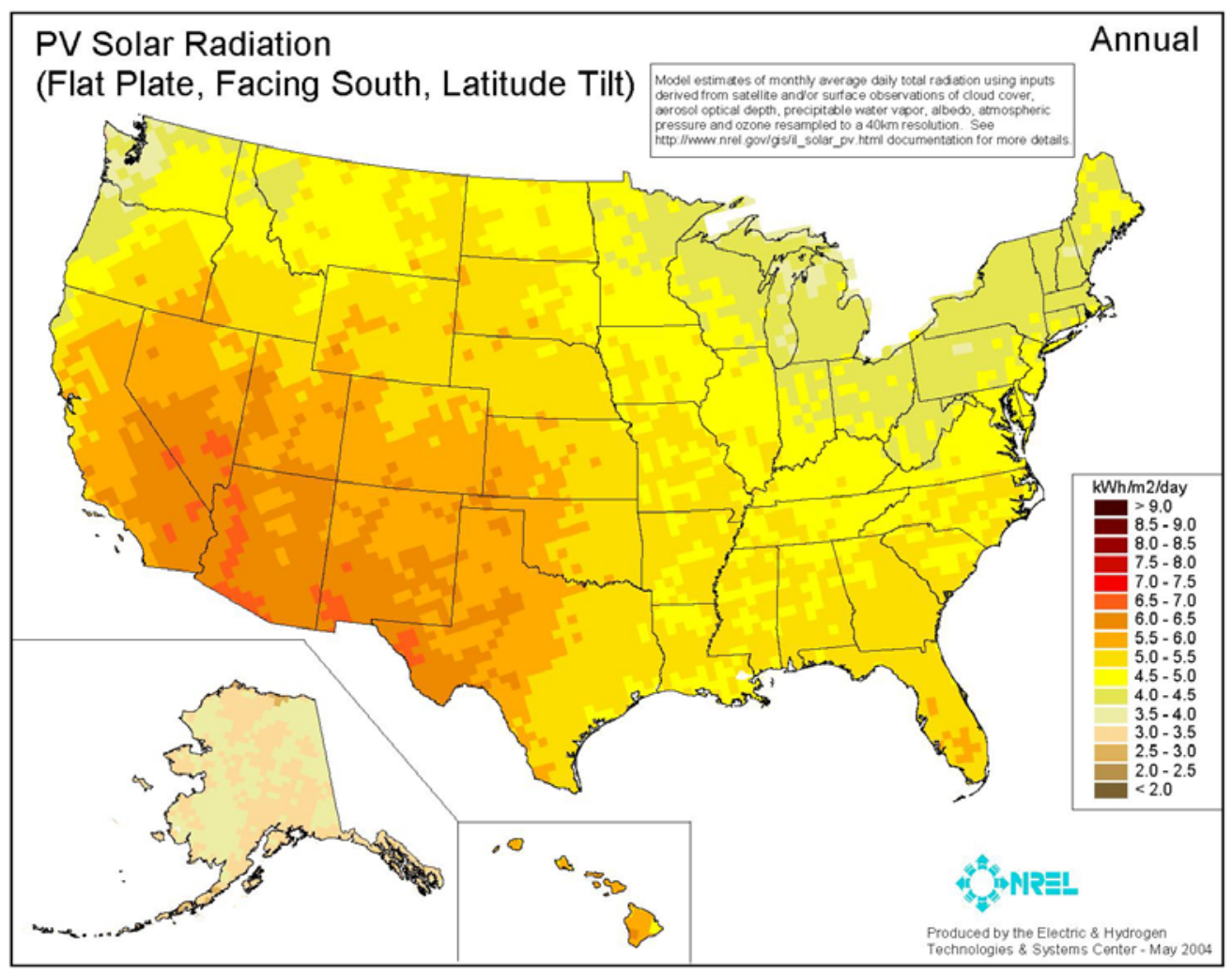

Figure 3.1 U.S. annual solar irradiance map

It doesn't take much imagination to see great opportunities for solar energy in the United States of America. The U.S. and Florida state annual average solar resources in $\mathrm{kWh} / \mathrm{m}^{2}$ per day in 2007 are shown in figure 3.1. Even in the $1930 \mathrm{~s}$, most new homes built in South Florida came equipped with their own solar water heaters. A 2004 study of small residential solar arrays by the Florida Solar Energy Center found that Florida has 85 percent of the maximum solar energy potential of any place in the country, at 6.5 kilowatt-hours per day.

Florida Power \& Light (FPL), a subsidiary of NextEra Energy Resources, Inc., launched 3 solar power plants in 2009 and 2010, making Florida the second largest supplier of utility-scale solar power in the country. In their lifetimes, these plants will prevent the emission of more than 3.5 million tons of greenhouse gases, equivalent to 
removing 25,000 cars from our roads each year. The Martin Next Generation Solar Plant, near Indiantown, Florida, is the first hybrid solar plant in the world. It's connected to a conventional power plant and directly offsets the burning of fossil fuels. The Martin plant is expected to cut Florida's oil consumption by 600,000 barrels, and save customers $\$ 178$ million in fuel costs over its lifetime [99].

\subsubsection{South Florida wind energy source analysis}

Wind energy is extracted from air flow using wind turbines to produce mechanical or electrical power. Wind power is the conversion of wind energy into a useful form of energy, such as using wind turbines to make electricity, wind mills for

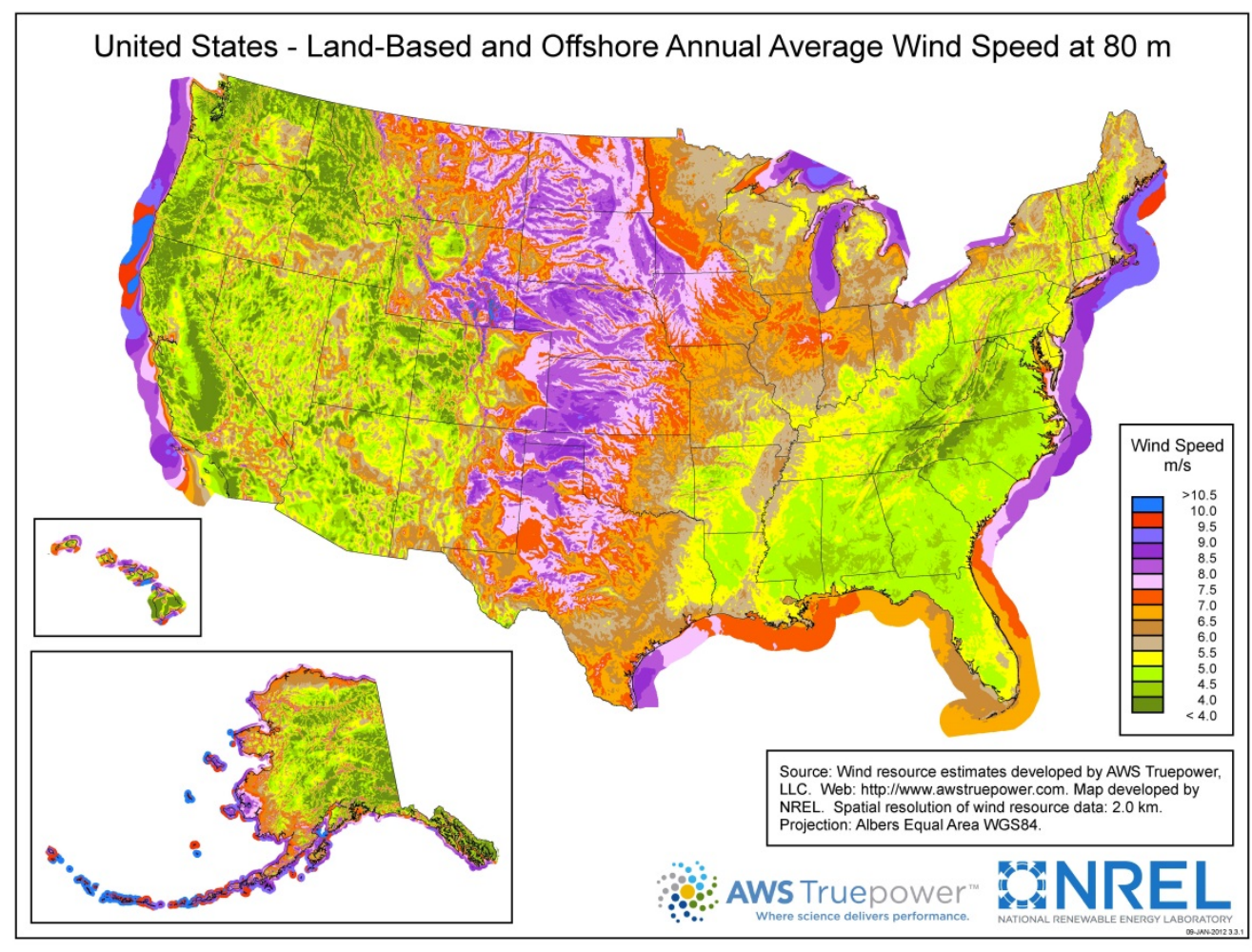

Figure 3.2 U.S. annual aver wind speed at $80 \mathrm{~m}$ 
mechanical power, wind pumps for pumping water or drainage, and sails to propel ships. Wind energy as an alternative to fossil fuels is plentiful, renewable, widely distributed, clean, produces no greenhouse gas emissions during operation, and uses little land. The effects on the environment are generally less problematic than those from other power sources.

Large wind farms consist of hundreds of individual wind turbines which are connected to the electric power transmission network; smaller facilities are used to provide electricity to isolated locations. Utility companies increasingly buy back surplus electricity produced by small domestic turbines.

Maps of the U.S. and Florida state annual wind potential capacity at $80-\mathrm{m}$ are

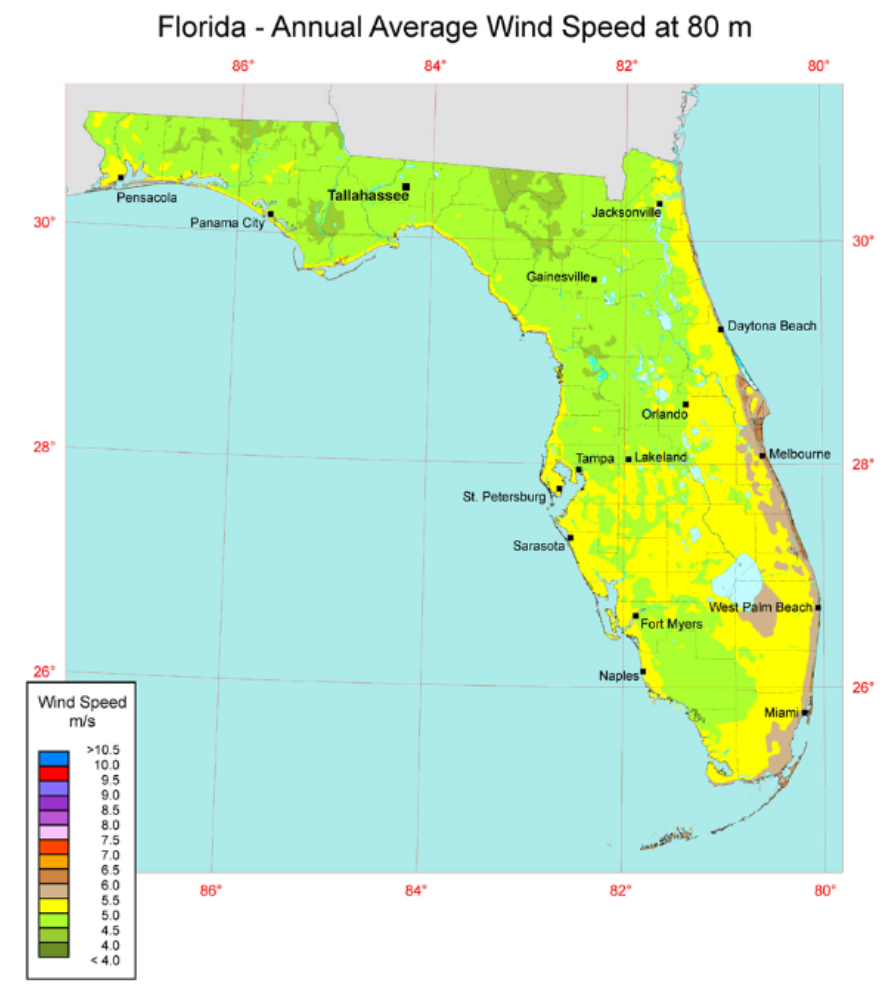

Figure 3.3 Florida annual average wind speed at $80 \mathrm{~m}$ 
shown in figure 3.2 and figure 3.3, respectively. The wind resource map shows the predicted mean annual wind speeds at an $80-\mathrm{m}$ height, presented at a spatial resolution of about 2 kilometers that is interpolated to a finer scale for display. Areas with annual average wind speeds around 6.5 meters per second and greater at $80-\mathrm{m}$ height are generally considered to have a resource suitable for wind development. Utility-scale, land-based wind turbines are typically installed between 80 - and 100-m high, although tower heights for new installations are increasing - up to $140 \mathrm{~m}$ - to gain access to better wind resources higher aloft [100].

\subsection{DC-DC Boost Converter with Maximum Power Point Tracking Ability for Solar Energy Harvesting}

\subsubsection{Photovoltaic panel MATLAB modeling}

For PV panels, the relation between output voltage and current is non-linear. Therefore, the terminal voltage of a PV panel is dependent on the amount of its output power. Also, the output power of a PV panel is dependent on solar irradiation and ambient temperature. On the other hand, a constant voltage level is needed for a DC bus connected to PV arrays. This imposes an imperative necessity of having a power conditioning unit as an interface between the PV panels and the DC bus connected to them. Figure 3.4 shows the electric characteristics of the PV panels. Fluctuations in the output current $(\Delta I)$ will generate fluctuations in the output voltage $(\Delta V)$, which may lead to fluctuations in the output power $(\Delta P)$. The figure indicates that any power fluctuations around the MPP will decrease the average power coming from the PV array. 


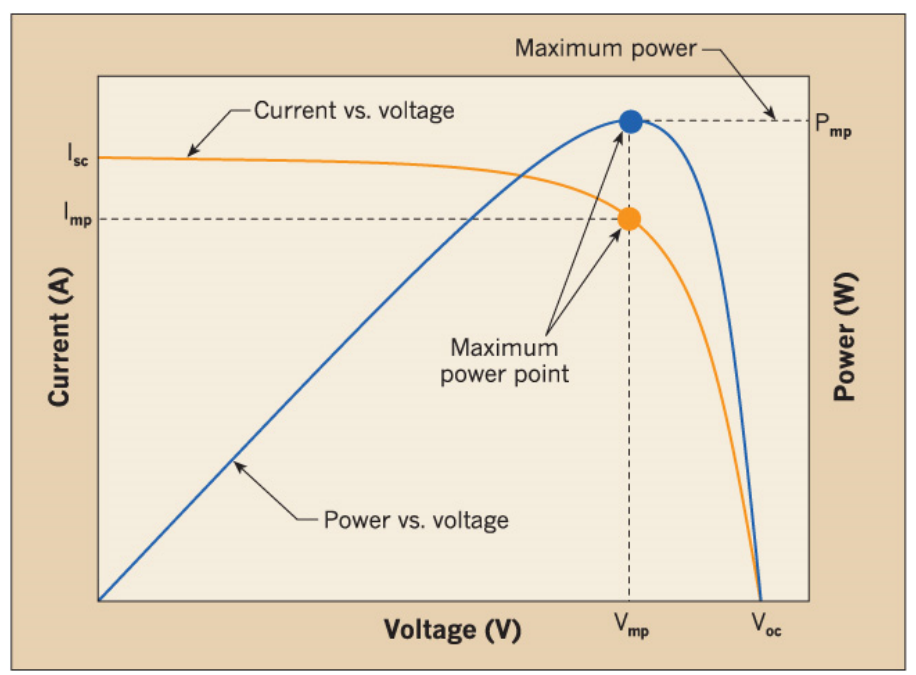

Figure 3.4 Electric characteristics of the PV panels

The PV panel can be viewed as a current source in parallel with a diode. Figure 3.5 shows the equivalent circuit of a PV panel with a load. Therefore, a Matlab model of a SunPower SPR-305-WHT solar cell with a 305W maximum output power PV panel is made based on the equivalent circuit. Figure 3.6 shows the non-linear P-V and I-V electric characteristics of a single SunPower SPR-305-WHT solar cell.

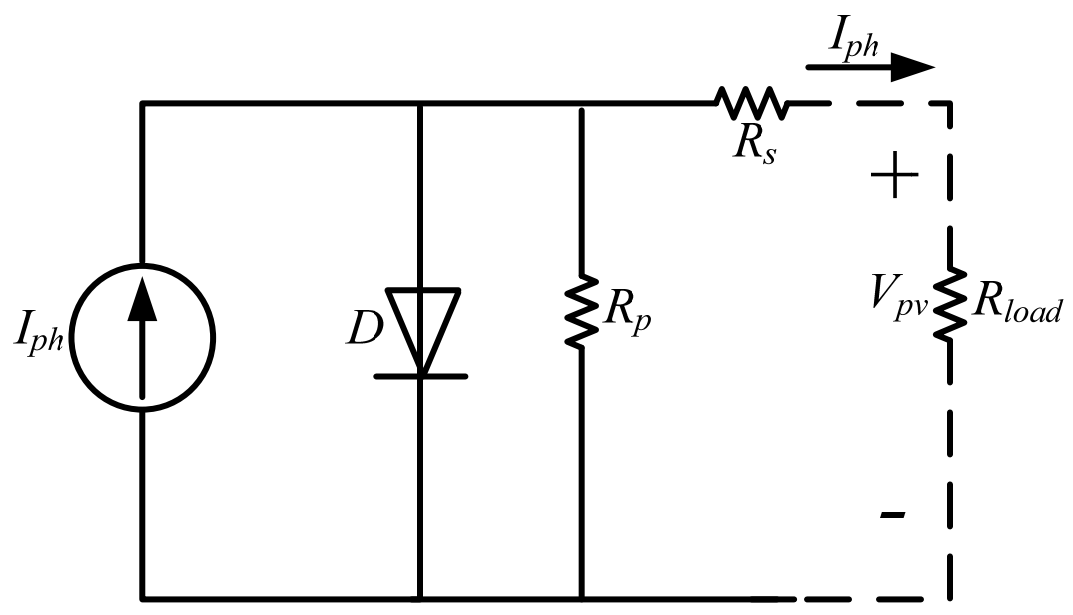

Figure 3.5 Equivalent circuit of a PV panel with a load 
Module type: SunPower SPR-305-WHT
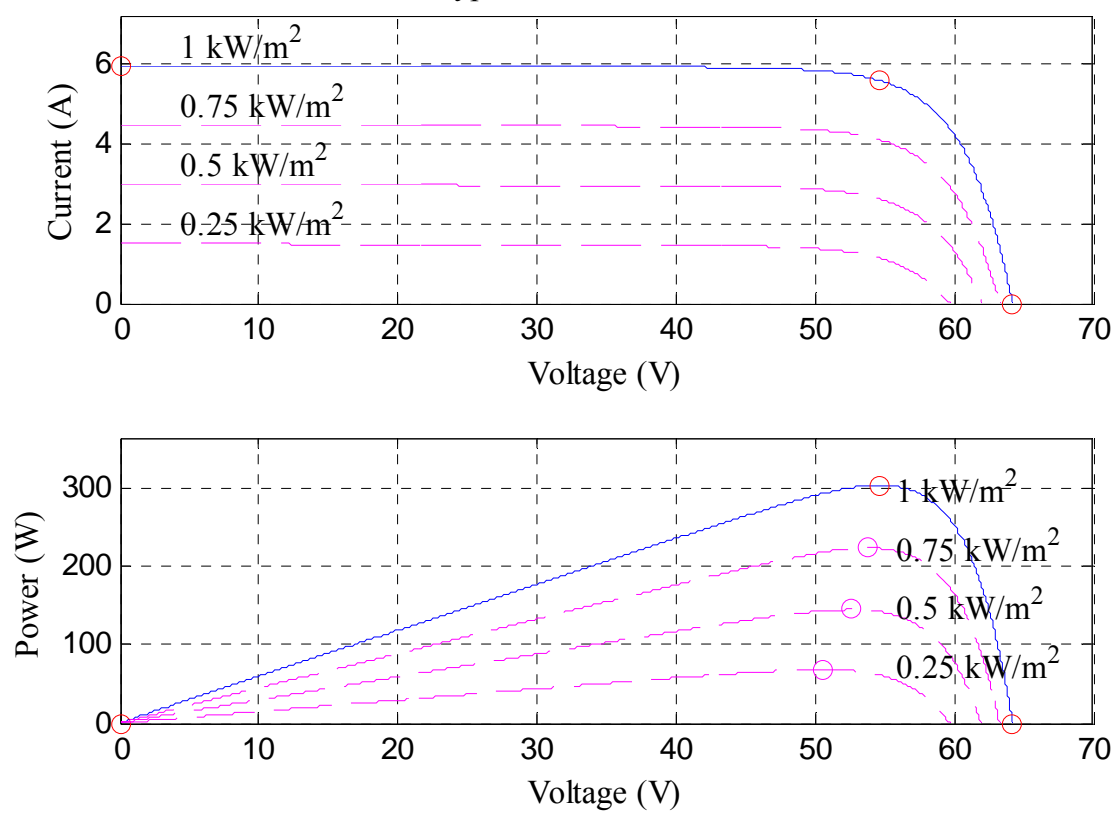

Figure 3.6 SunPower SPR-305-WHT solar cell P-V and I-V electric characteristics

\subsection{2. $\quad$ DC-DC boost converter MATLAB modeling}

A DC-DC boost converter is one of the most commonly used techniques to regulate the output power and voltage of a PV panel and inject the energy into the DC

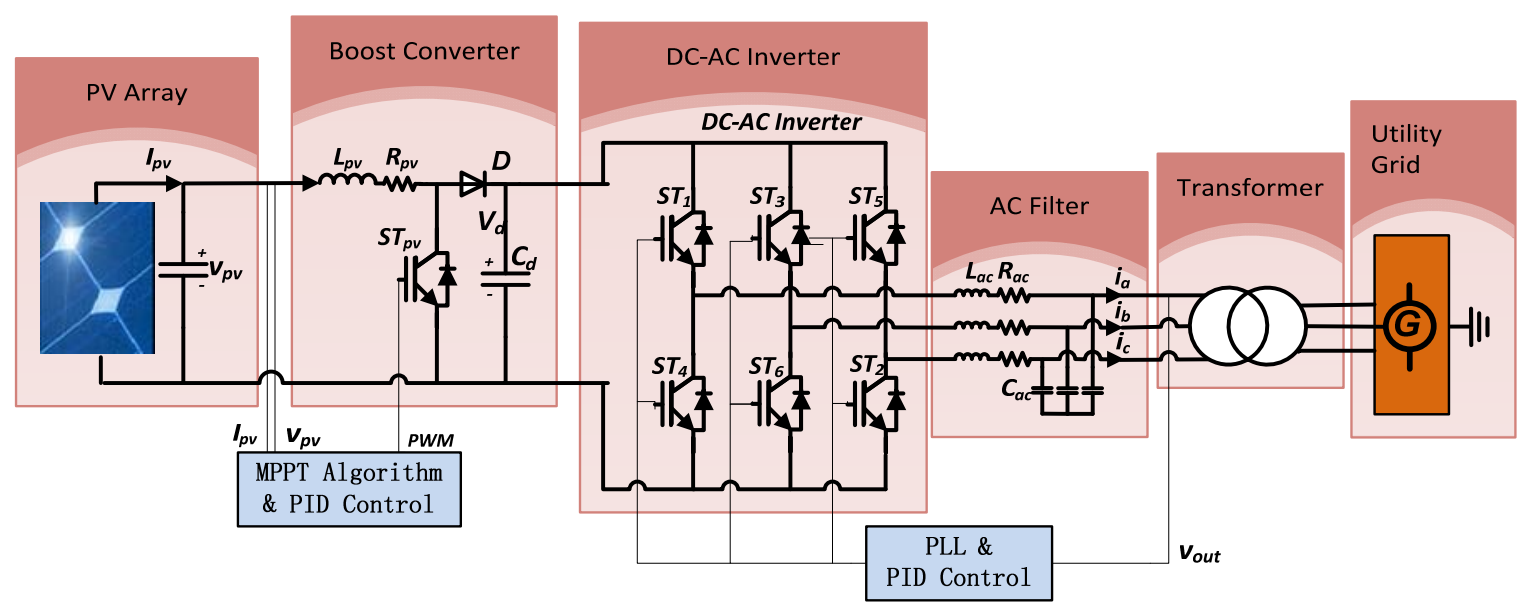

Figure 3.7 Grid connected PV farm system configuration. 
side of the hybrid power system. Usually the DC side of the hybrid power system is connected to the AC subsystem through a bidirectional AC-DC inverter. In this way, the power generated from the PV can be injected to the utility grid. The grid connected PV farm system configuration is shown in figure 3.7.

With proper design and control, a DC-DC converter can effectively regulate output voltage even under input voltage and output current variations. But these variations have a range which depends on the design and control of the converter and the load conditions on the DC bus. The DC-DC boost converter circuit topology is shown in figure 3.8. The high voltage side of the DC-DC converter has a capacitive $C_{d}$ interface to the DC bus. This capacitor has the function of regulating the output voltage and current.

The key principle that drives the boost converter is the tendency of an inductor to resist changes in current by creating and destroying a magnetic field. In a boost converter, the output voltage is always higher than the input voltage. The schematic of a boost power stage is shown in figure 3.9 (a) and (b). When the switch is closed, current flows

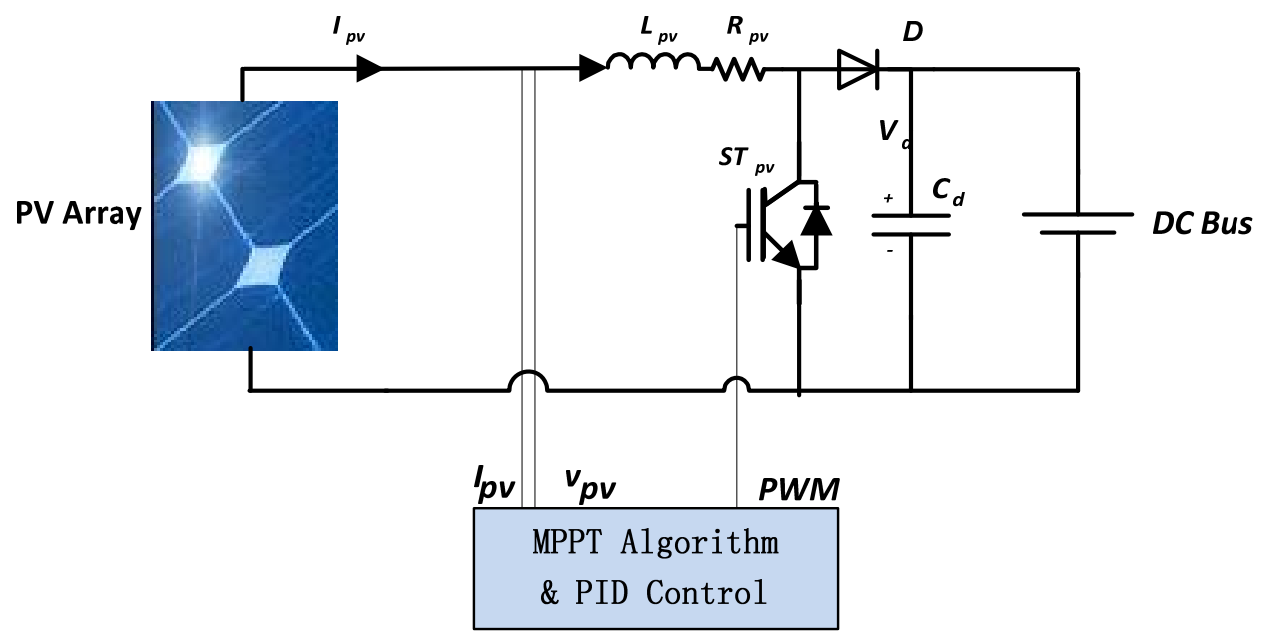

Figure 3.8 DC-DC boost converter circuit topology 
through the inductor in a clockwise direction and the inductor stores some energy by generating a magnetic field. Polarity of the left side of the inductor is positive. When the switch is opened, current will be reduced as the impedance is higher. The magnetic field previously created will be destroyed to maintain the current flow towards the load. Thus the polarity will be reversed (meaning the left side of inductor will be negative now). As a result, two sources will be in series causing a higher voltage to charge the capacitor through the diode $\mathrm{D}$.

For general purpose, this chapter only discusses the DC-DC converter working in

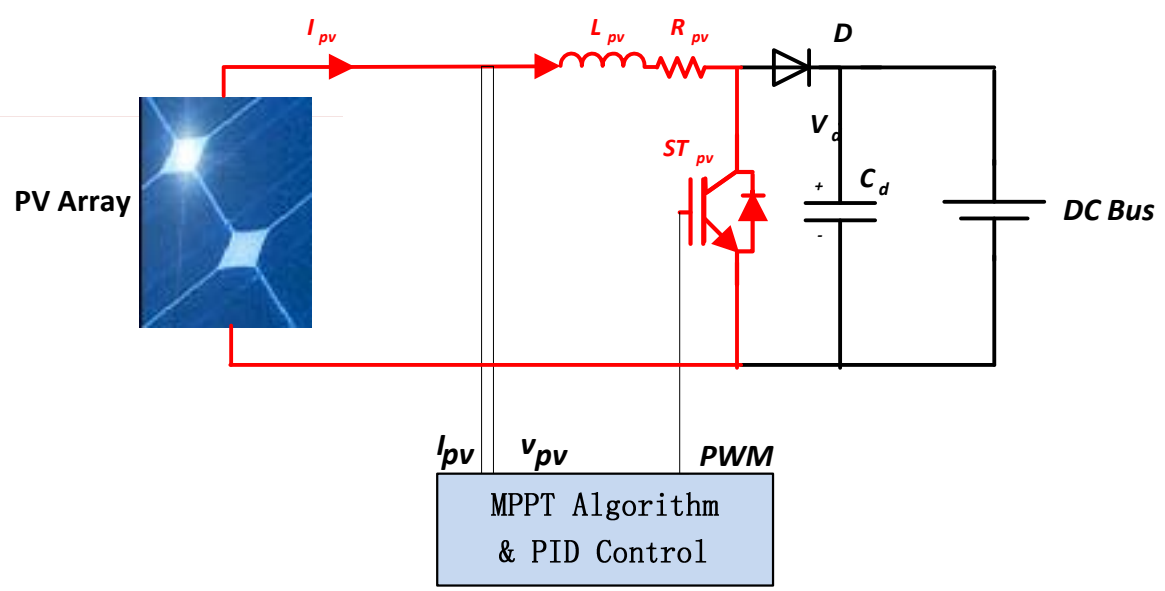

(a)

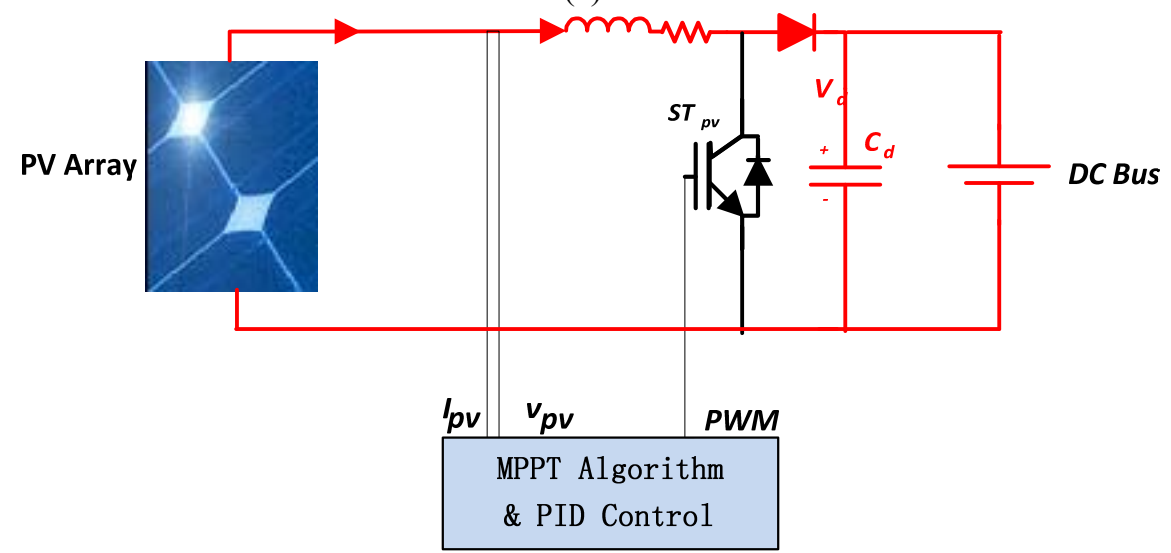

(b)

Figure 3.9 Circuit configuration during different states of the power electronic switch: (a) During On state $(0<\mathrm{t}<\mathrm{DT})$, (b) During OFF state $(\mathrm{DT}<\mathrm{t}<\mathrm{T})$. 
continuous mode. When a boost converter operates in continuous mode, the current through the inductor $I_{L}$ never falls to zero. In the case of an ideal converter operating in steady conditions, the output voltage can be calculated as follows:

During the On-state, the switch $\mathrm{S}$ is closed, which makes the input voltage $V_{i}$ appear across the inductor, which causes a change in current $I_{L}$ flowing through the inductor during a time period $t$ by the formula:

$$
\frac{\Delta I_{L}}{\Delta t}=\frac{V_{i}}{L}
$$

At the end of the On-state, the increase of $I_{L}$ is therefore:

$$
\Delta I_{L O n}=\frac{1}{L} \int_{0}^{D T} V_{i} d t=\frac{D T}{L} V_{i}
$$

where $D$ is the duty cycle. It represents the fraction of the commutation period $T$ during which the switch is on. Therefore $D$ ranges between 0 and 1 .

During the Off-state, the switch $\mathrm{S}$ is open, so the inductor current flows through the load. If we consider zero voltage drop in the diode, and a capacitor large enough for its voltage to remain constant, the evolution of IL is:

$$
V_{i}-V_{o}=L \frac{d I_{L}}{d t}
$$

Therefore, the variation of $I_{L}$ during the Off-period is:

$$
\Delta I_{L O f f}=\int_{D T}^{T} \frac{\left(V_{i}-V_{o}\right)}{L} d t=\frac{\left(V_{i}-V_{o}\right)(1-D) T}{L}
$$

As we consider that the converter operates in steady-state conditions, the amount of energy stored in each of its components has to be the same at the beginning and end of a commutation cycle. In particular, the energy stored in the inductor is given by: 


$$
E=\frac{1}{2} L I_{L}^{2}
$$

So, the inductor current has to be the same at the start and end of the commutation cycle. This means the overall change in the current (the sum of the changes) is zero:

$$
\Delta I_{L O n}+\Delta I_{L O f f}=0
$$

Substituting $\Delta I_{\text {Lon }}$ and $\Delta I_{\text {Loff }}$ by their expressions yields:

$$
\Delta I_{L O n}+\Delta I_{L O f f}=\frac{V i D T}{L}+\frac{(V i-V o)(1-D) T}{L}=0
$$

This can be written as:

$$
\frac{V_{o}}{V_{i}}=\frac{1}{1-D}
$$

Which in turn reveals the duty cycle to be:

$$
D=1-\frac{V_{i}}{V_{o}}
$$

The above expression shows that the output voltage is always higher than the input voltage. This is why this converter is sometimes referred to as a step-up converter.

\subsubsection{Photovoltaic panel maximum power point tracking}

To improve the PV system performance and increase the efficiency, the DC-DC converter connected between the PV system and DC bus should have the maximum power point tracking (MPPT) function. There are several proposed PV system MPPT methods [101]-[103]. The popular MPPT algorithms can be classified into two main categories: direct and indirect methods. Indirect methods use either a database containing the parameters and data that show the characteristics of the PV panel at certain environmental conditions, or some mathematical functions achieved by the experience of 
the estimation of the maximum power point. Direct methods find the maximum power point based on the PV output voltage and current together with the relationship of the changes in them to the changing of the output power.

The indirect methods include "look-up table method", "open circuit voltage method", "short-circuit method", "curve-fitting method", "open-circuit voltage photovoltaic test cell method", and more. The indirect methods have the advantage that it doesn't need to measure voltage or current in real time operation, therefore the converter working with indirect methods are usually simple with no feedback loop and the cost is relatively low. But the drawback for indirect methods is that in most cases a prior evaluation of the PV panel is required, and it's not easy to reach the precise maximum power point in most of these situations.

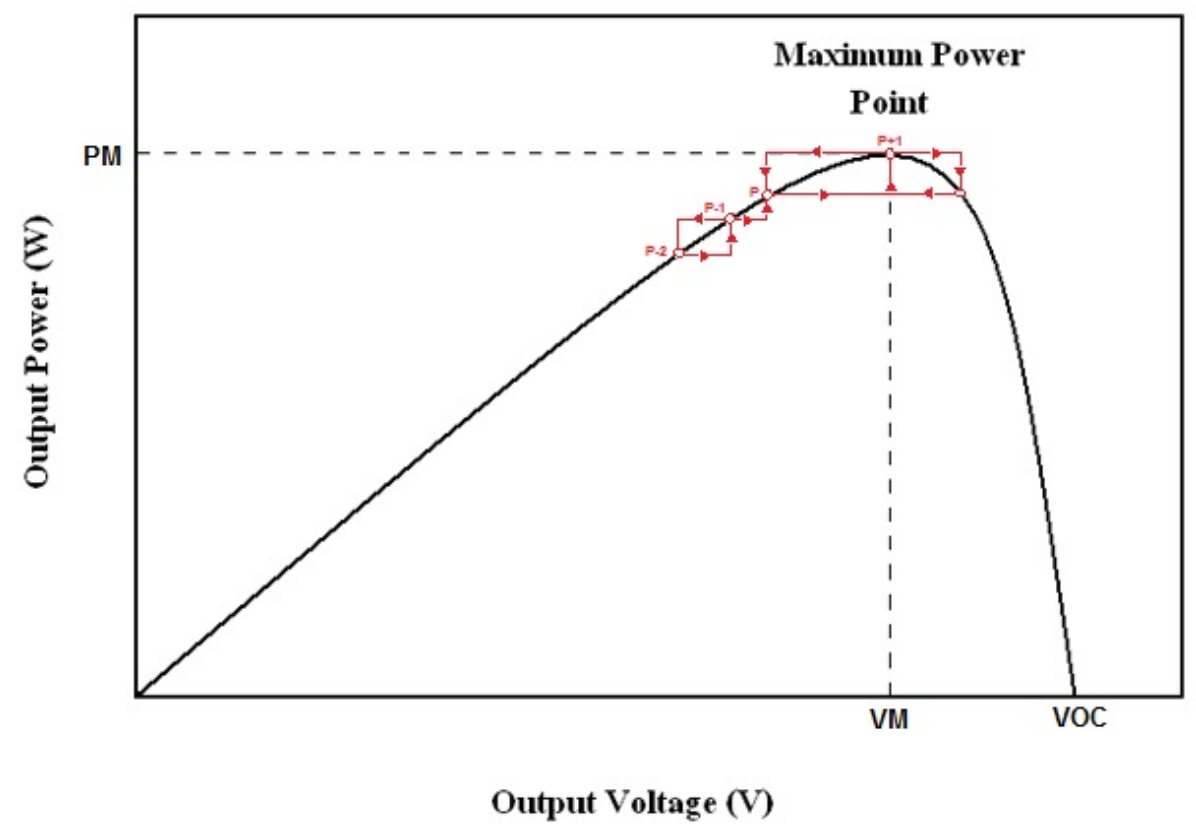

Figure 3.10 Perturbation and observe MPPT operating point path. 
The direct methods include "differentiation method", "artificial intelligence method", and "P\&O method". The direct methods do not rely on prior experience, which means the variance of the environmental variables won't influence the performance. However, the feedback loop is needed for the direct methods, which will increase the boost converter complexity and cost.

In this study, the perturbation and observe (P\&O) method is used to track the maximum power point. The $\mathrm{P} \& \mathrm{O}$ operating point path for maximum power point tracking is shown in figure 3.10. The algorithm utilizes the PV farm output current and voltage to calculate the power. The values of the voltage and power at the $k^{\text {th }}$ iteration $\left(P_{k}\right)$ are stored, then the same values are measured and calculated for the $(k+1)^{\text {th }}$ iteration

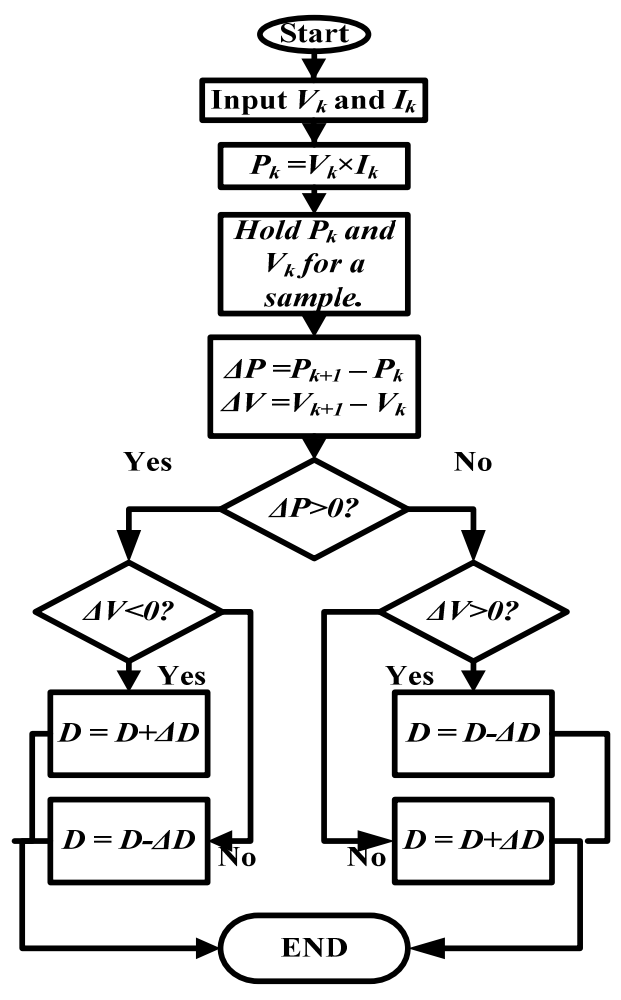

Figure 3.11 Flow chart of P\&O MPPT method 
$\left(P_{k+1}\right)$. The power difference between the two iterations $(\Delta P)$ is calculated. The converter should increase the PV panel output voltage if $\Delta P$ is positive and decrease the output voltage if $\Delta \mathrm{P}$ is negative, which finally will adjust the duty cycle. The $\mathrm{PV}$ panel reaches the maximum power point when $\Delta \mathrm{P}$ is approximately zero. The flow chart of the $\mathrm{P} \& \mathrm{O}$ MPPT algorithm is given in figure 3.11.

\subsubsection{Photovoltaic panel emulator design}

Installation of a PV system requires an accurate design and evaluation test for the performance of its modules before using it in a system. This could be fulfilled with high accuracy in the laboratory without using commercial PV modules by using a PV emulator. The PV emulator introduces a suitable tool which easily permits emulating PV system components with different parameters, teaching its characteristics, and training researchers in this field.

In this work, a $6 \mathrm{~kW}$ programmable $\mathrm{PV}$ emulator is designed that has the capability of emulating commercial PV modules with different parameters and under different environmental conditions (irradiance or temperature change), as well as constructing a PV array based on a desired number of series and parallel modules. The PV emulator is implemented using a real-time algorithm representing the PV array mathematical model and generating the reference output power from a programmable DC power supply. The PV model is simulated in MATLAB and interfaced to the real world through dSpace using an evolution graphic user interface (GUI). This laboratory PV emulator is tested with real-time execution of PV models for the steady state and dynamic operating conditions. The block diagram representation of the proposed PV emulator is shown in figure 3.12. It essentially consists of a programmable DC power supply 
(Programmable Magna Power Supply XR series), Matlab/Simulink package, dSpace1104 with control desk software installed on personal computer (PC), and resistive load with different values. The detailed hardware experimental results of the PV emulator will be given in Chapter 8 section 8.51 .

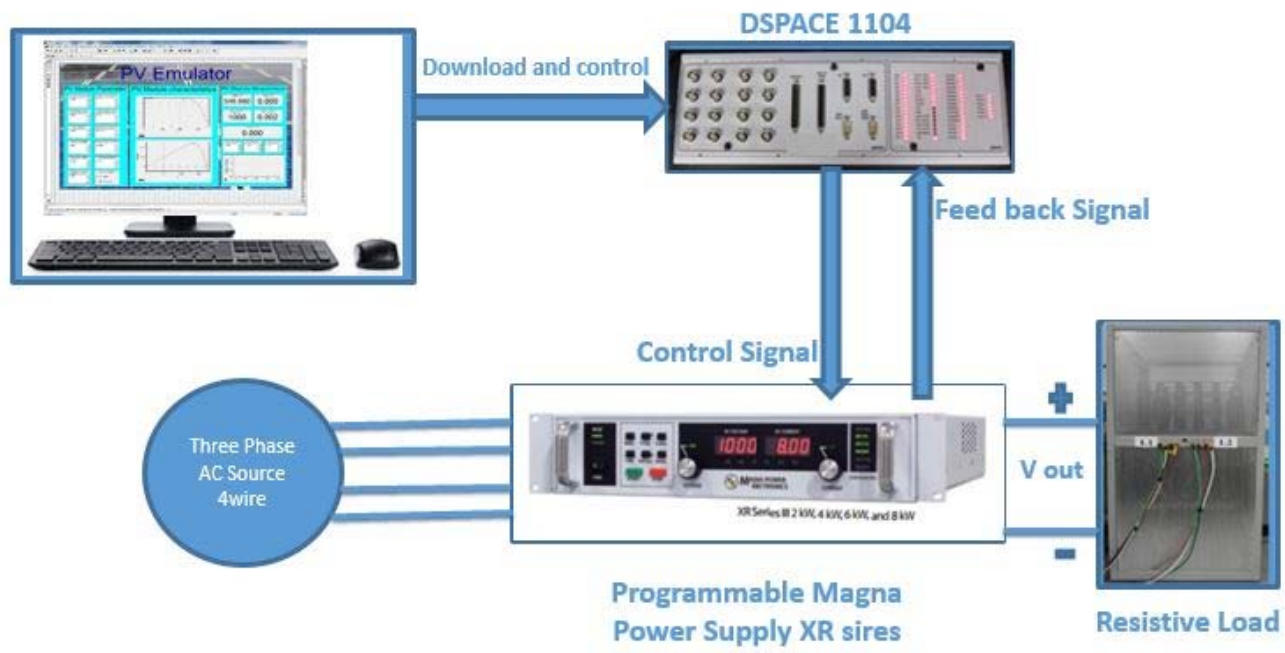

Figure 3.12 Block diagram for the proposed PV Emulator

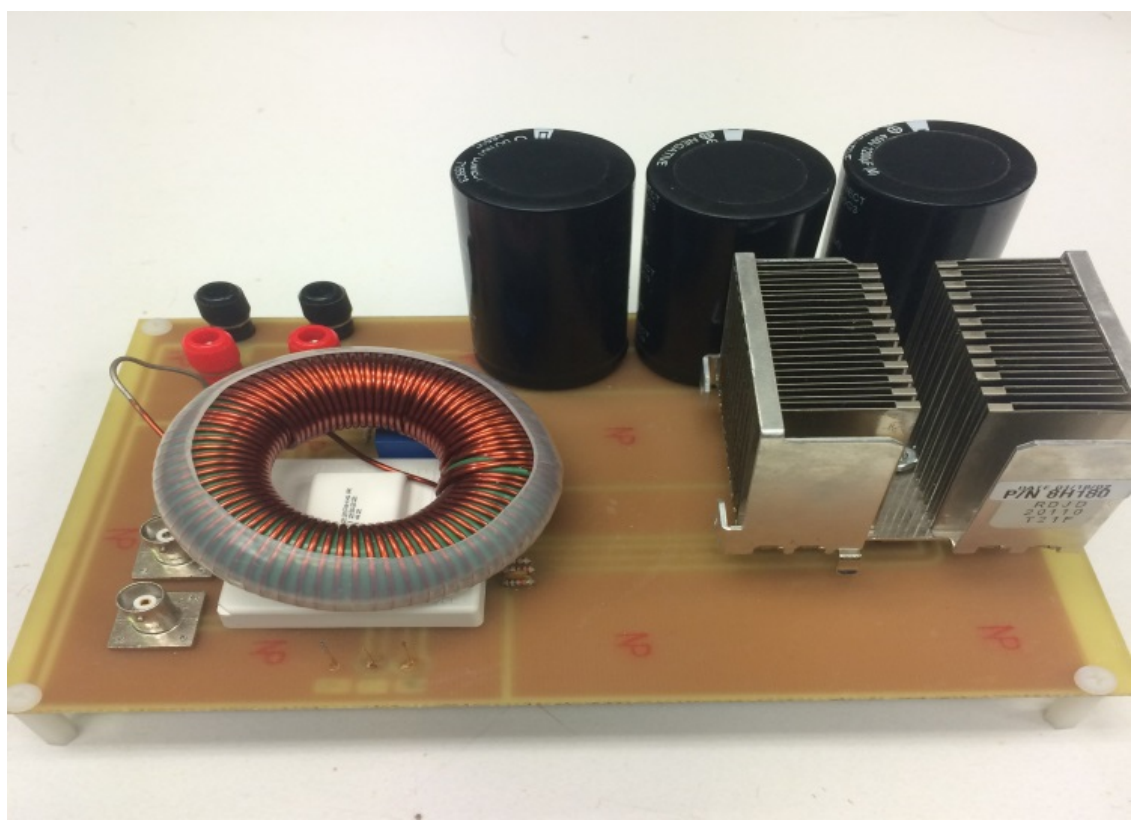

Figure 3.13 DC-DC boost converter hardware 


\subsubsection{DC-DC boost converter hardware design}

A PCB based DC-DC boost converter is designed for the laboratory scaled PV emulator. The DC-DC boost converter hardware is shown in figure 3.13. It contains a SK45GB063 IGBT module, and the power rating is $3 \mathrm{~kW}$. The capacity of the capacitor on the high voltage side is $3600 \mathrm{uF}$, and the inductance of the coupled inductor is $3 \mathrm{mH}$. This DC-DC converter is operated with a $5 \mathrm{kHz}$ switching frequency. The performance results will be given in Chapter 8 Section 8.3.

\subsection{Power conversion topologies with wind turbines}

The power extracted from wind is derived from the airflow speed reaching the wind turbine and the speed leaving from it. Only part of the full energy can be captured from the wind for energy generation, quantified by the power coefficient $C_{p}$, which is the relationship between the possible power extractions to the total of the wind power.

With different wind speeds, the wind energy conversion system should be operated in different states. If the wind speed is below the cut-in wind speed or above the cut-out wind speed, the wind turbine works in the no load state. When the wind turbines are in the full load state, the output power must be regulated at rated power by changing the pitch angle. To maximize the output power, the tip speed ratio needs to be controlled. As the rotor speed must change according to the wind intensity, the speed control of the turbine has to command low speed at low winds and high speed at high winds, so as to follow the maximum power operating point as indicated in figure. 3.14. It is observed 
that the maximum power output occurs at different generator speeds for different wind velocities.

Wind turbines use a doubly-fed induction generator (DFIG) consisting of a wound rotor induction generator and "back-to-back" IGBT-based PWM converters. The stator winding is connected directly to the $60 \mathrm{~Hz}$ grid while the rotor is fed at variable frequency through the back-to-back converter. The optimum turbine speed producing maximum mechanical energy for a given wind speed is proportional to the wind speed.

The basic block diagram of DFIG with converters is shown in figure 3.15 , where $V_{r}$ is the rotor voltage and $V_{g c}$ is grid side voltage. The back-to-back converter is basically a PWM converter which uses sinusoidal PWM technique to reduce the harmonics present in the wind turbine driven DFIG system. Here, $C_{\text {rotor }}$ is the rotor side converter and $C_{\text {grid }}$

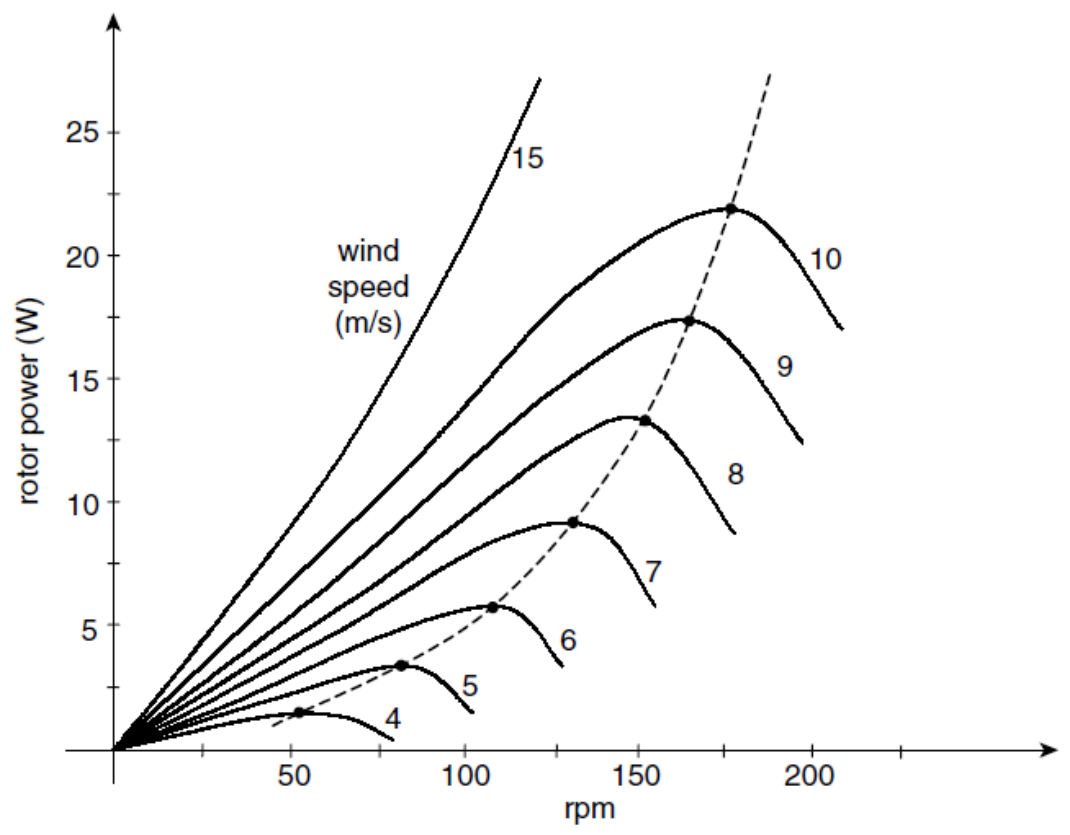

Figure 3.14 Wind turbine rotation versus power characteristic at different wind speed. 
is the grid side converter. To control the speed of the wind turbine, gear boxes or electronic control can be used.

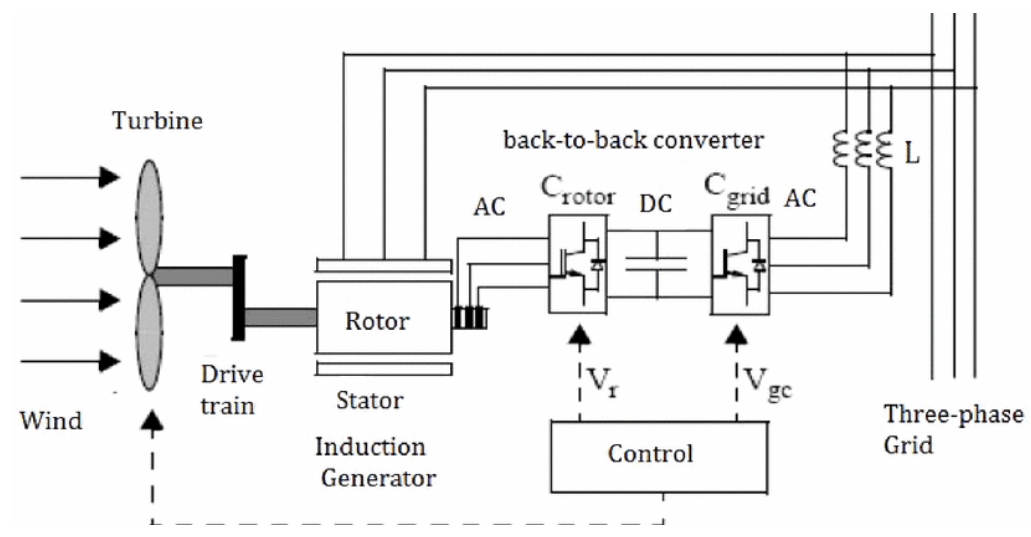

Figure 3.15 Basic diagram of doubly fed induction generator with back-to-back converter

The stator is directly connected to the AC mains, while the wound rotor is fed from the Power Electronics Converter via slip rings to allow the DFIG to operate at a variety of speeds in response to changing wind speed. Indeed, the basic concept is to interpose a frequency converter between the variable frequency induction generator and fixed frequency grid. The DC capacitor linking stator- and rotor-side converters allows the storage of power from the induction generator for further generation. To achieve full control of grid current, the DC-link voltage must be boosted to a level higher than the amplitude of grid line-to-line voltage. The wind-side converter controls the active power through MPPT, while the grid-side converter controls the DC voltage and reactive power to the grid.

The first power conversion stage has to be controlled to maximize the energy extracted from the wind turbine. The open loop MPPT is mostly used in commercial power converters, but a closed loop algorithm can also be adopted when the turbine characteristics are unknown. For every wind speed, there is a particular turbine speed that 
corresponds to the maximum power conversion (optimal tip speed ratio). A wind converter must control the turbine rotational speed in order to follow the optimal tip speed ratio when the wind speed changes, therefore the MPPT algorithm must be implemented. With the knowledge of the turbine characteristics and the wind speed (that can be obtained with an anemometer on the turbine nacelle), a speed-controlled generator can harvest the maximum power from the wind. Also, to extract the maximum power from the wind energy, turbine blades should change their speed as the wind speed changes.

The slip power can flow in both directions, i.e. to the rotor from the supply and from supply to the rotor and hence the speed of the machine can be controlled from either the rotor or stator side converter in both super and sub-synchronous speed ranges.

\subsection{Renewable energy sources impact to hybrid power systems}

Large scale wind and solar generation will affect the physical operation of the grid. The variability of wind and solar regimes across resource areas, the lack of correlation between wind and solar generation volatility and load volatility, and the size and location of the wind plants relative to the system in most US states suggests that impacts on regulation and load profile requirements resource smoothing will be large at above $20 \%$ penetration levels.

The largest barrier to renewable integration in the USA is sufficient transmission facilities, associated cost-allocation in the region to access the renewable resources, and connecting these resources to load centers. Other key barriers include environmental pressure, technical interconnection issues such as forecasting, dispatch ability, low 
capacity factors, and intermittency impacts on the regulation services of renewable resources. With the integration of wind and solar generation the output of fossil fuelplants needs to be adjusted frequently to cope with fluctuations in output. Some power stations will be operated below their maximum output to facilitate this, and extra system balancing reserves will be needed. Efficiency may be reduced as a result with an adverse effect on emissions. At high penetrations (above 20\%), wind and solar energy may need to be 'spilled' or curtailed because the grid cannot always utilize the excess energy. The impacts of renewable energy sources to the utility grid can be summarized as follows:

- Impacts of Renewable Energy: wind and solar generation both experience intermittency, a combination of non-controllable variability and partial unpredictability, and depend on resources that are location-dependent.

- Non-controllable variability: wind and solar output varies in a way that generation operators cannot control, because wind speeds and available sunlight may vary from moment to moment, affecting moment-to moment power output. This fluctuation in power output results in the need for additional energy to balance supply and demand on the grid on an instantaneous basis, as well as ancillary services such as frequency regulation and voltage support.

- Partial unpredictability: the availability of wind and sunlight is partially unpredictable. A wind turbine may only produce electricity when the wind is blowing, and solar PV systems require the presence of sunlight in order to operate. Actual wind power can differ from forecasts, even when multiple forecast scenarios are considered. Unpredictability can be managed through 
improved weather and generation forecasting technologies, the maintenance of reserves that stand ready to provide additional power when renewable energy generation produces less energy than predicted, and the availability of dispatchable load to "soak up" excess power when renewable energy generation produces more energy than predicted.

- Location dependence: the best wind and solar resources are based in specific locations and, unlike coal, gas, oil or uranium, cannot be transported to a generation site that is grid optimal. Generation must be co-located with the resource itself, and often these locations are far from the places where the power will ultimately be used. New transmission capacity is often required to connect wind and solar resources to the rest of the grid. Transmission costs are especially important for offshore wind resources, and such lines often necessitate the use of special technologies not found in land-based transmission lines.

Therefore, to accelerate the development of future hybrid AC-DC power systems with high penetration of renewable energy sources, more research is needed in the following areas:

- New and in-depth focus on system planning. Steady-state and dynamic considerations are crucial.

- Accurate resource and load forecasting becomes highly valuable and important. 
- Voltage support. Managing reactive power compensation is critical to grid stability. This also includes dynamic reactive power requirements of intermittent resources.

- Evolving operating and power balancing requirements. Sensitivity to existing generator ramp rates to balance large scale wind and solar generation, providing regulation and minimizing start stop operations for load following generators.

- Increased requirements on ancillary services. Faster ramp rates and a larger percentage of regulation services will be required which can be supplied by responsive storage facilities.

- Equipment selection. Variable Speed Generation (VSG) turbines and advanced solar inverters have the added advantage of independent regulation of active and reactive power. This technology is essential for large-scale renewable generation.

- Strong interconnections. Several large energy pump-storage plants are available in Switzerland that are used for balancing power. Larger regional control areas make this possible.

\subsection{Conclusion}

In this chapter, we begin by analyzing the U.S and Florida State wind and solar energy characteristics. After that, the methods of harvesting solar energy from PV panels in hybrid power systems through DC-DC boost converters and MPPT technology is presented. In addition, the methodology of designing a library scaled PV emulator and 
DC-DC boost converter hardware is given. Also, the basic description of power conversion topologies of wind turbines is given with the information of how to maximize the output power from wind turbine power generation systems. Based on the above information, the renewable energy sources impact to the hybrid power system and how to overcome those challenges are described in detail. 
4. Optimal Renewable Energy Farm and Energy Storage Sizing Methodology for Future Hybrid Power System

\subsection{Introduction}

Fossil fuels as energy resources are becoming scarce and have already led to worldwide issues at the environmental, industrial, economic, and societal levels. This has generated an increased interest in clean energy throughout the globe. Power generation based on renewable energy sources, such as solar and wind, will play an increasingly important role in energy production as our energy reserves diminish [104], [105]. The U.S government has set itself the goal that by the fiscal year 2020, 20 percent of the total amount of electric energy consumed by each agency during any fiscal year thereafter shall be renewable energy [106].

Due to the large variances of solar irradiance and wind speed, the power generated from a renewable farm consisting of PV and wind turbines is considered intermittent [107]. Therefore, without properly planning the scale of the renewable energy farm, the utility grid may be badly influenced by the power generated by the renewable energy farm in the local micro grid (MG). This problem can be solved by utilizing energy storage devices such as batteries and ultra-capacitors together with an energy management algorithm as well as high accuracy weather and load forecasting techniques [108]. A carefully designed hybrid power system with reasonable sizing of the 
renewable energy farm and energy storage system can greatly improve the power system performance and reduce the total cost.

Various optimal sizing methods for renewable energy farms and their accompanying energy storage systems have been proposed. The optimization process can be classified into single objective and multi-objective methods. In [34], a renewable energy farm sizing optimization method together with the economic analysis is proposed for a 50,000 plug-in electric vehicles network energy requirement. However, the process that limits the renewable energy farm's power fluctuation impact to the utility grid is not analyzed. In [109], a method based on the cost benefit analysis for optimal sizing of an energy storage system in a $\mathrm{MG}$ is proposed, but the appropriate sizing of the renewable energy farm is not given. In addition, the energy storage system only included the use of batteries, which may fail in high power fluctuation situations. In [110], an optimal sizing method for a wind-solar-battery hybrid power system is proposed for both stand-alone and grid-connected modes, however, the ultra-capacitor is not considered.

This chapter proposes a novel optimization method for sizing a renewable energy farm consisting of batteries and ultra-capacitors in a hybrid power system. The combination of ultra-capacitors with batteries is an emerging practice in advanced power electronic systems and a superior configuration scheme is crucial to deploy them effectively with the high penetration of renewable energy sources and critical loads in future power systems. The proposed sizing method fully utilizes the energy generated from the renewable energy farm and limits power fluctuation within the utility grid, improving grid stability and reducing construction and maintenance costs. This two-step optimization process appropriately sizes the renewable energy farm and the energy 
storage system by using a genetic algorithm (GA). Regional historical data of the solar irradiance, wind speed, and local load profile of Key West, Florida is used to establish the first cost function for optimizing the combination of PV and wind power based on the entire year's daily energy difference between the renewable energy farm and twenty percent of the local load. With the optimized renewable energy farm size, a second cost function is designed to get the optimal combination of battery and ultra-capacitor sizes to lessen the impact caused by the renewable energy farm. A case study of a hybrid power system located in Key West, Florida is presented to verify the advantages of the proposed optimal sizing method.

This chapter is organized as follows. The description of modern energy storage systems is given in section 4.2. The system's overall description along with the models of the PV, wind turbine, battery, and ultra-capacitor is introduced in section 4.3. The renewable energy farm optimal sizing methodology is presented in Section 4.4. In Section 4.5, the power filter design for power gaps dispatch is described. The energy storage system optimization and a case study of the power system in Key West, U.S.A is presented in Section 4.6 to verify the performance of the optimization method proposed in this chapter. Finally, a conclusion is given in Section 4.7.

\subsection{Modern Energy Storages Classification}

\subsubsection{Battery}

An electric battery is a device consisting of one or more electrochemical cells that convert stored chemical energy into electrical energy. Each cell contains a positive terminal, or cathode, and a negative terminal, or anode. Electrolytes allow ions to move 
between the electrodes and terminals, which allows current to flow out of the battery to perform work. Rechargeable batteries can be discharged and recharged multiple times and the original composition of the electrodes can be restored through reverse current.

Rechargeable batteries are used for automobile starters, portable consumer devices, light vehicles (such as motorized wheelchairs, golf carts, electric bicycles, and electric forklifts), tools, and uninterruptible power supplies.

Grid energy storage applications use rechargeable batteries for load leveling, where they store electric energy for use during peak load periods, and for renewable energy uses, such as storing power generated from photovoltaic arrays during the day to be used at night. By charging batteries during periods of low demand and injecting energy to the grid during periods of high electrical demand, load-leveling helps eliminate the need for expensive peaking power plants and helps reduce the cost of generator operations.

Current Li-Ion batteries offer an energy density of about $160 \mathrm{Wh} / \mathrm{kg}$.

\subsubsection{Fuel cell}

A fuel cell is a device that converts the chemical energy from fuel into electricity through a chemical reaction with oxygen or another oxidizing agent. Hydrogen produced from the steam methane reforming of natural gas is the most common fuel but for greater efficiency, hydrocarbons such as natural gas and alcohols like methanol can be used. Fuel cells are different from batteries in that they require a continuous source of fuel and oxygen/air to sustain the chemical reaction whereas in a battery the chemicals present in the battery react with each other to generate an electromotive force. Fuel cells can produce electricity continuously for as long as these inputs are supplied. 
Stationary fuel cells are used for commercial, industrial and residential primary and backup power generation. Since fuel cell electrolyzing systems do not store fuel themselves, but rather rely on external storage units, they can be successfully applied in large-scale energy storage, such as rural areas. Combined heat and power fuel cell systems are used to generate both electricity and heat for homes, office buildings and factories. The system generates constant electric power (selling excess power back to the grid when it is not consumed), and at the same time produces hot air and water from the waste heat.

Fuel Cells offer energy densities of about $2500 \mathrm{Wh} / \mathrm{kg}$.

\subsubsection{Ultra-capacitor}

An ultra-capacitor is a high-capacity electrochemical capacitor with capacitance values up to 10,000 Farads at 1.2 Volts that bridges the gap between electrolytic capacitors and rechargeable batteries. They typically store 10 to 100 times more energy per unit volume or mass than electrolytic capacitors, can accept and deliver charge much faster than batteries, and tolerate many more charge and discharge cycles than rechargeable batteries. They are, however, 10 times larger than conventional batteries for a given charge.

Ultra-capacitors are used in applications requiring many rapid charge/discharge cycles rather than long term compact energy storage. They are utilized within cars, buses, trains, cranes and elevators, where they are used for recovering energy from braking, short-term energy storage or burst-mode power delivery.

Ultra-capacitors can stabilize voltage for power lines. Wind and photovoltaic systems exhibit fluctuating loads evoked by clouds that ultra-capacitors can buffer within 
milliseconds. This helps stabilize grid voltage and frequency, balance supply and demand of power and manage real or reactive power.

Current ultra-capacitors offer energy density reaching $15 \mathrm{Wh} / \mathrm{kg}$.

\subsubsection{Flywheel}

A flywheel is a rotating mechanical device that is used to store rotational energy. Flywheels have a significant moment of inertia and thus resist changes in rotational speed. The amount of energy stored in a flywheel is proportional to the square of its rotational speed. Energy is transferred to a flywheel by applying torque to it, thereby increasing its rotational speed, and hence its stored energy. Conversely, a flywheel releases stored energy by applying torque to a mechanical load, thereby decreasing the flywheel's rotational speed.

Flywheels are often used to provide continuous energy in systems where the energy source is not continuous. In such cases, the flywheel stores energy when torque is applied by the energy source, and it releases stored energy when the energy source is not applying torque to it.

A flywheel may also be used to supply intermittent pulses of energy at transfer rates that exceed the abilities of its energy source, or when such pulses would disrupt the energy supply, such as in a public electric network. This is achieved by accumulating stored energy in the flywheel over a period of time, at a rate that is compatible with the energy source, and then releasing that energy at a much higher rate over a relatively short time.

Flywheels used in electric power backup feature energy densities of $43 \mathrm{Wh} / \mathrm{kg}$. 


\subsection{Local Load and Renewable Energy Sources Pattern Analysis}

The hybrid power system considered in this study is shown in figure 4.1. The system has loads and generators in both $\mathrm{AC}$ and $\mathrm{DC}$ sides which are linked by a bidirectional AC-DC inverter. The system includes energy harvesting from solar photovoltaics and a wind turbine generator farm, all of which are sized in terms of area and number of wind turbines required to serve the load pattern in the region. Energy storage devices such as battery banks and ultra-capacitors are connected to the DC bus through bidirectional DC-DC converters. By properly controlling these power electronic devices, energy can be transferred from energy storage devices to the AC and DC sides of the hybrid power system and vice versa. In this way, the energy storage devices can serve as an energy buffer to fill up the energy gap between the local load and the output power of the renewable energy farm.

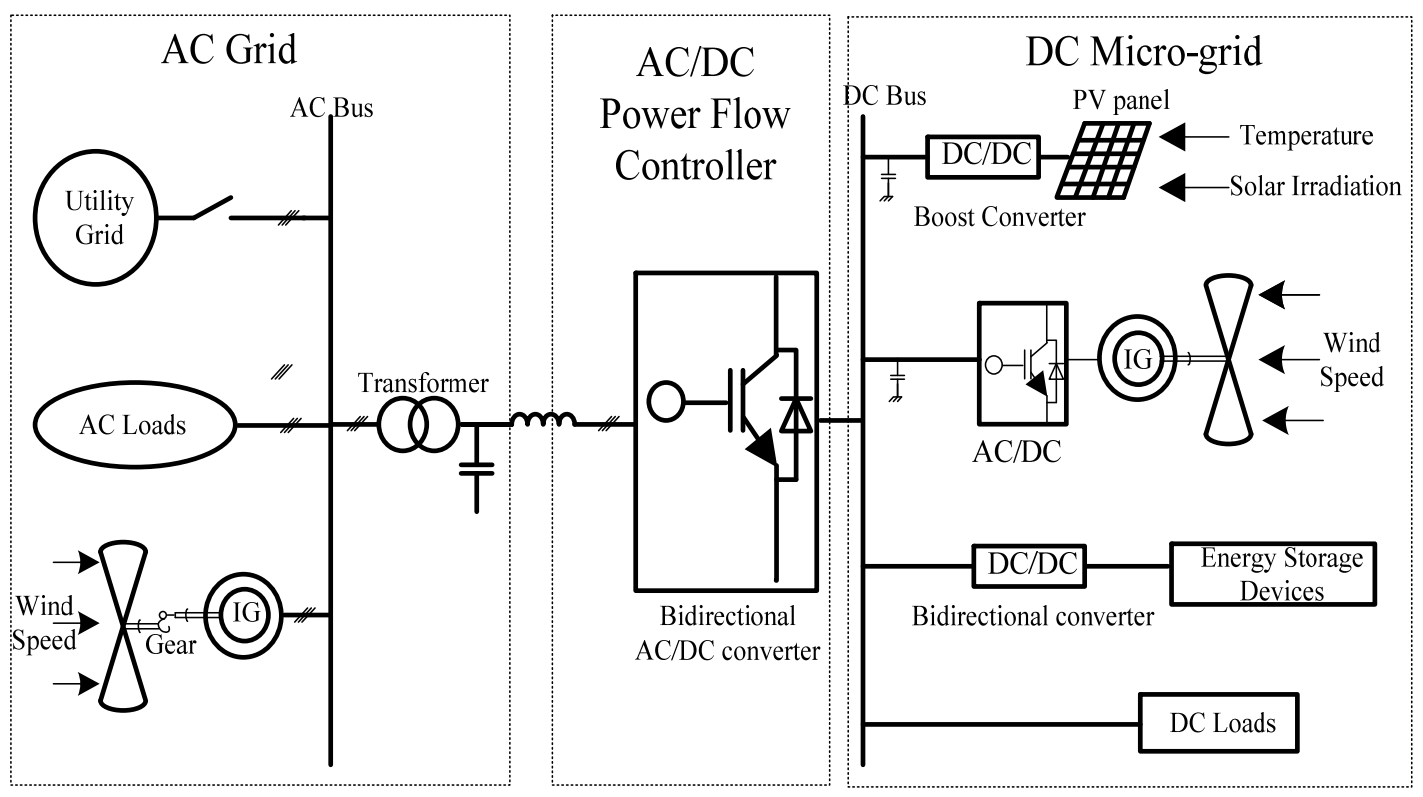

Figure 4.1 Overview of the proposed hybrid power system 


\subsubsection{Key West local load pattern analysis}

The Key West local load pattern in the year 2005 shown in figure 4.2 is used in this study. The daily load follows a specific pattern. Because of the small daily temperature variations throughout the entire year, there is only one daily peak that occurs around 12:00 PM. Also, during the night from 22:00 PM to 7:00 AM, there is a load valley. Note that the sudden drops in the Key West load are due to a loss of information or massive outages which were a result of the 2005 hurricane season.

Since the daily load follows the specific pattern with one peak and one valley, a renewable energy farm system together with an energy storage system can be established to generate and store energy to shave the load peak and fill the load valley.

\subsubsection{Key West renewable energy sources historical analysis}

The Key West local solar irradiance pattern in the year 2005 shown in figure 4.3

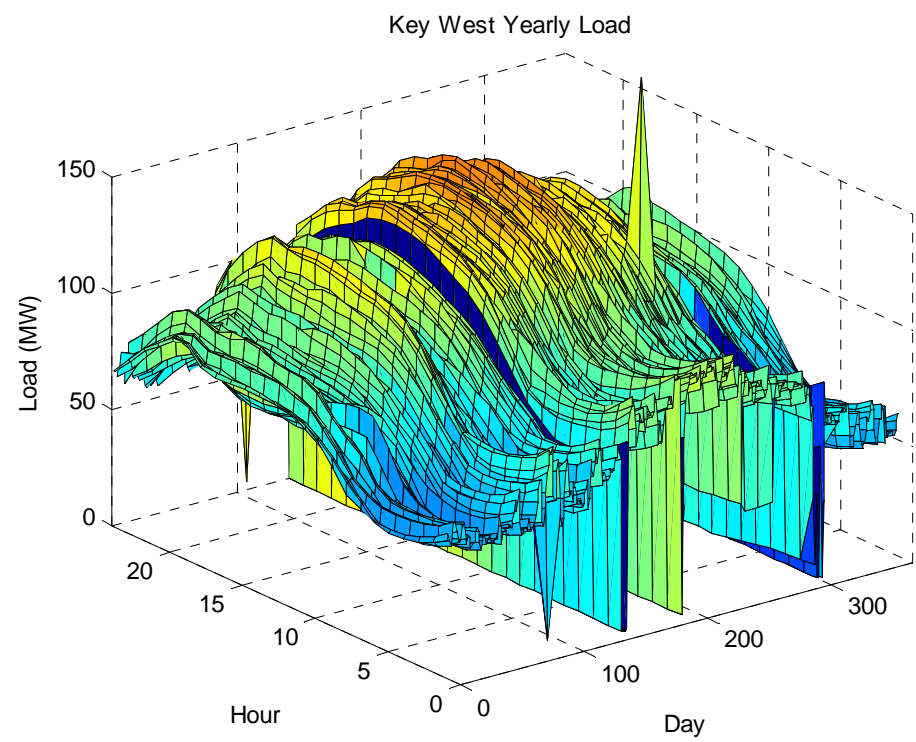

Figure 4.2 Key West local load patter in 2005 


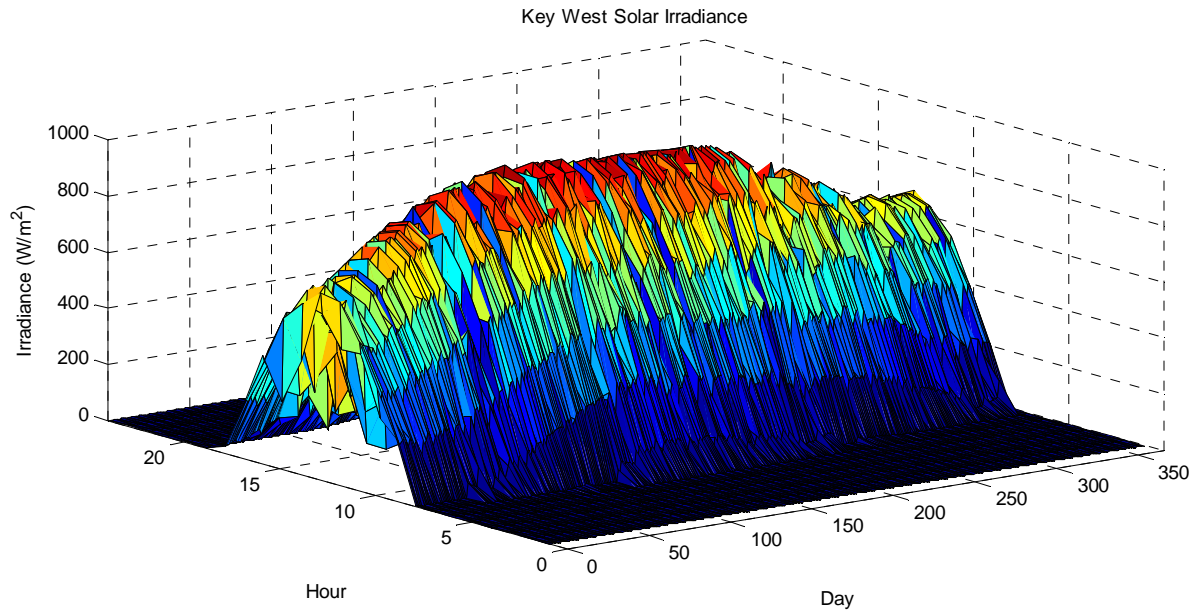

Figure 4.3 Key West local solar irradiance pattern in 2005

is used in this study. Key West is affluent of solar energy and the solar irradiance also follows a daily pattern. Because of this, the solar energy should be utilized to support a certain part of the local load.

The ideal power output $P_{P V i d e a l}$ in $k W$ neglecting temperature correction is:

$$
P_{\text {PVideal }}=\eta S I
$$

where the power of the cell is calculated by the efficiency $\eta$ multiplied by the PV surface area $S$ in $\mathrm{m}^{2}$ and the irradiance $I$ in $\mathrm{kW} / \mathrm{m}^{2}$ shown in equation (4.1). The temperature coefficient is set to be $0.5 \%$ /C [111]. The hourly Key West solar irradiance data for the year 2005 is shown in figure 4.4 .

A simple temperature correction component is made based on the temperature coefficient:

$$
P_{P V}=\eta S I\left[1-0.005\left(T_{m}-25\right)\right]
$$

where $T_{m}$ presents the module temperature. By introducing nominal operating cell temperature given by the panel manufacturer, an estimation of the resulting module temperature can be formulated based on the ambient temperature [112]: 


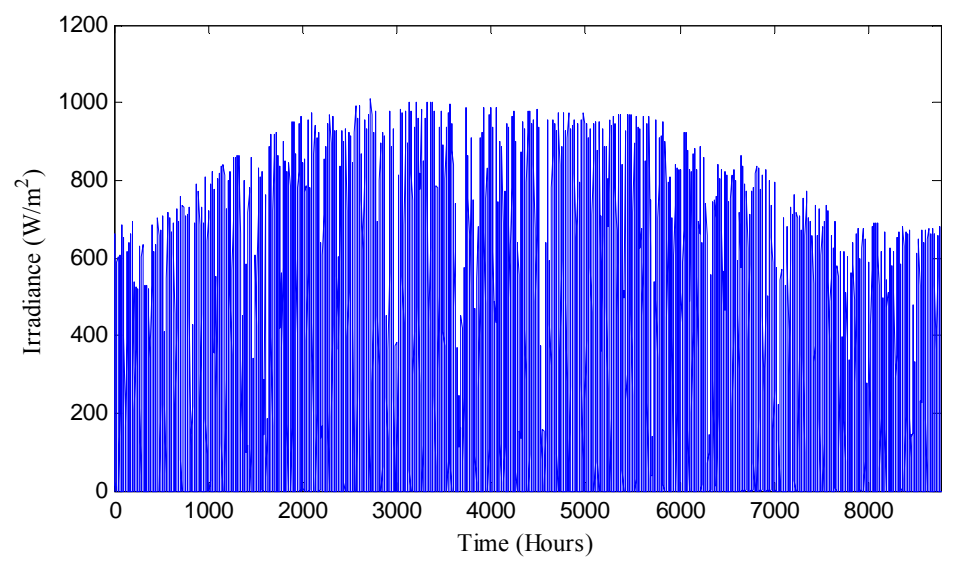

Figure 4.4 Hourly Key West solar irradiance data, 2005

$$
T_{m}=T_{a m b}+\left(\frac{N O C T-20}{0.8}\right)
$$

where $T_{a m b}$ represents the ambient temperature. The Key West hourly temperature data for the year 2005 is shown in figure 4.5. Following experimental research from NREL, values for normal operating cell temperature (NOCT) are generally in the region of $45.3^{\circ}$ $\mathrm{C}$ to $49.8^{\circ} \mathrm{C}$, thus, using an average NOCT of $47.6 \mathrm{C}$ results in following equation (4.4):

$$
T_{m}=T_{a m b}+34.5 I
$$

Plugging into (4.2) yields:

$$
P_{p v}=\eta S I\left[1-0.005\left(T_{a m b}+34.5 I-25\right)\right]
$$

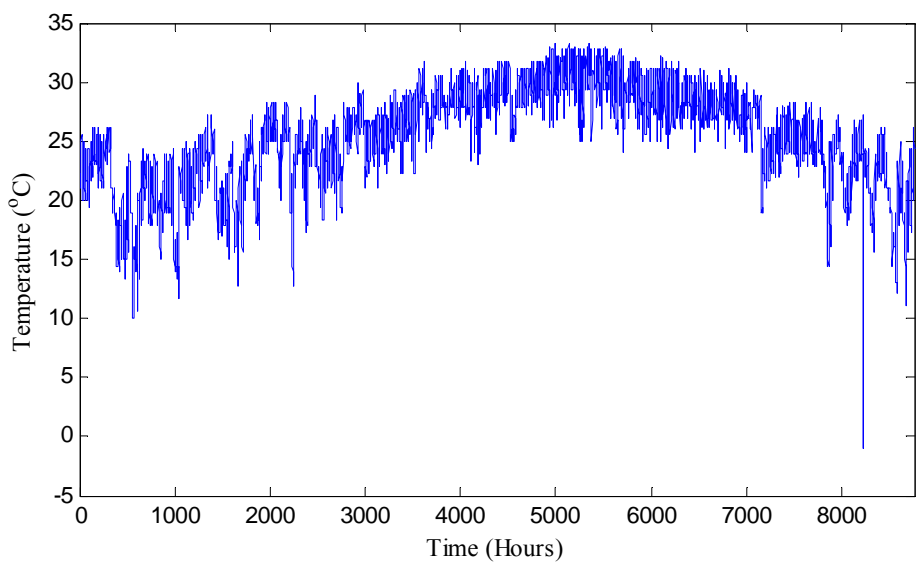

Figure 4.5 Key West hourly temperature data, 2005 
However, during the night there is no solar energy, and for some extreme weather like in the hurricane season, the whole area may be covered by clouds for several days. Therefore, solar energy may fail to support sufficient sustainable energy, and for reliability reasons, the hybrid power system shouldn't only rely on solo renewable energy sources. As a result, wind energy is also utilized for this case of study.

The Key West local offshore wind speed pattern in the year 2005 shown in figure 4.6 is used in this study. The average wind speed is above 10 meter per second.

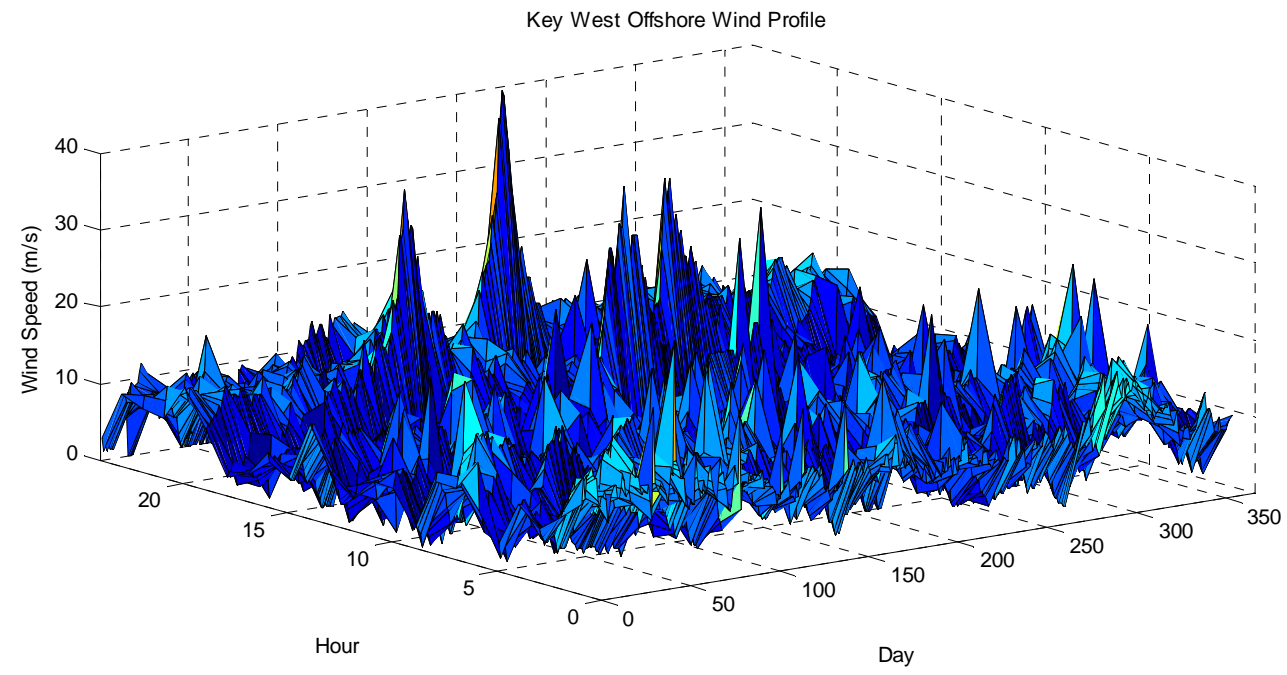

Figure 4.6 Key West local offshore wind speed pattern in the year 2005

The wind turbine power model used in this study provides a simple relationship of mechanical power based on the kinetic energy of the wind. Power is represented by its fundamental definition of a mass of air moving at a velocity $v$ passing through the blade coverage area $A$ shown in equation (4.6):

$$
\text { Pwind }=\frac{1}{2} \rho A v^{3}
$$


where $\rho$ is the air density in $\mathrm{kg} / \mathrm{m}^{3}$ which is further modified by taking into account the ambient air temperature $T_{a m b}$. The Key West hourly wind speed data for the year 2005 is shown in figure 4.7. To depict the variance in the air density:

$$
\rho=\frac{P \cdot M \cdot W .}{R\left(273.15+T_{\text {amb }}\right)}
$$

where $\mathrm{P}$ is the absolute pressure ( $1 \mathrm{~atm}), \mathrm{M} . \mathrm{W}$. is the molecular weight of the gas $(0.02897 \mathrm{~kg} / \mathrm{mol})$, and $\mathrm{R}$ is the ideal gas constant at $8.2056 x 10^{-5} \mathrm{~m}^{3} \cdot \mathrm{atm} \cdot \mathrm{K}^{-1} \cdot \mathrm{mol}^{-1} \cdot \rho$ is corrected as equation (4.7):

$$
\rho=\frac{353 P}{\left(273.15+T_{a m b}\right)}
$$

Plugging into (4.6) yields:

$$
P_{\text {wind }}=\frac{176.5 P}{\left(273.15+T_{\text {amb }}\right)} A v^{3}
$$

With the utilization of wind energy, the hybrid power system can get renewable energy support during the night and during cloudy days when PV panels can't provide much power. The combination of solar and wind energy can greatly improve the reliability of the hybrid power system.

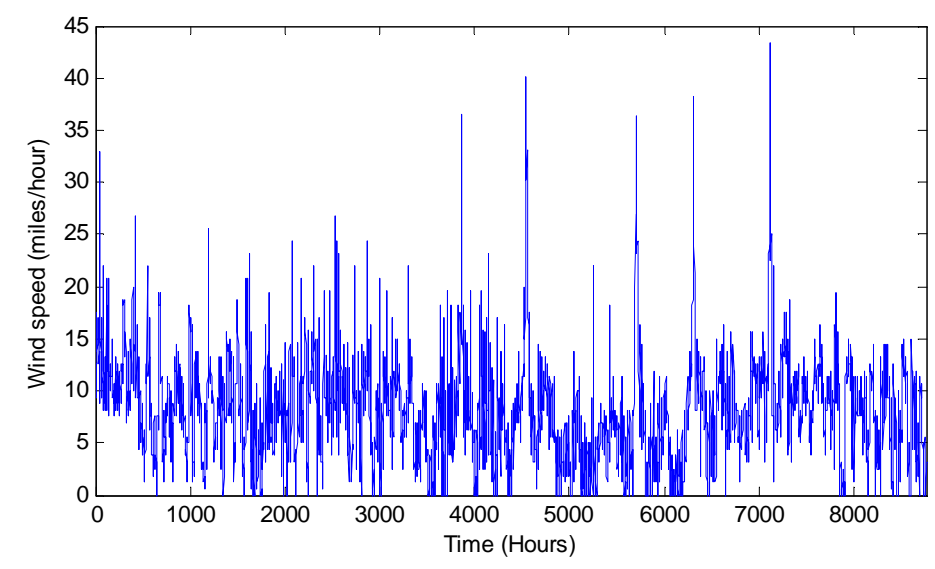

Figure 4.7 Key West hourly temperature data, 2005 


\subsection{Renewable Energy Farm Scale Optimization}

To optimize the size of the renewable energy farm to supply enough power to support around twenty percent of the total local load, historical solar irradiance and wind speed data is analyzed together with the daily local load pattern. The daily energy generated by the solar panels can be calculated using equation (4.10), in which $A$ is the solar panel size. The NMC CHSM6612P-310W photovoltaic panel model is used in this chapter to build the solar energy farm. The unit price is $\$ 263.50 / \mathrm{m}^{2}$ with a cost of only $\$ 0.83 / \mathrm{W}$.

$$
E_{\text {sloar }}=A \int_{t=0}^{t=24} P_{p v}(t) d t
$$

For the output of the wind farm, The Nordex N29-250 kW wind turbine model is used to determine the output power generated by the wind under different wind speed situations. The wind turbine unit price is $\$ 140,000$. The total daily energy output from the wind farm is calculated in (4.6) where $N$ indicates the number of wind turbines, $v_{(t)}$ is the wind speed at time $t$ and $P_{\text {wind }}\left(v_{(t)}\right)$ represents the single turbine output power at time $t$.

$$
E_{\text {wind }}=N \times \int_{t=0}^{t=24} P_{\text {wind }}\left(s_{(t)}\right) d t
$$

\subsubsection{Renewable energy farm scale optimization cost function design}

To build a renewable energy farm with an energy output that matches the twenty percent of the local load with minimum construction and maintenance cost, a cost function regarding the size of the renewable energy farm is given in (4.12).

$$
\begin{aligned}
f_{1}(A, N)= & \alpha \sum_{d=1}^{d=365}\left|E_{\text {solar }}(d)+E_{\text {wind }}(d)-E_{\text {load }}(d)\right| \\
& +(1-\alpha)\left(A \times P V u+N \times W T G_{u}\right)
\end{aligned}
$$


where $\alpha$ is the performance-cost weight, $P V_{u}$ is the solar panel unit price, and $W T G_{u}$ is the wind turbine generator unit price. This cost function contains two parts; the first part is the annual energy difference between the renewable energy farm output energy and a certain percentage of the local load. In this study, we choose $20 \%$ of Key West's local load. The smaller the first part of the equation is, the better the performance of the renewable energy will be. In extreme weather conditions, the renewable energy farm's daily energy output can perfectly match $20 \%$ of the local load which results in the first part having a cost function of 0 . However, this type of over fitting may generate a solution with a very high cost, which may not be utilized because of the high price. As a result, a second part is considered in the cost function. The second part represents the cost of establishing the renewable energy farms. The smaller this part of the equation is, the lower the cost of the whole renewable energy farm is. A performance-cost weight variable $a$ is designed to balance the cost and the performance of the optimization process. If $a$ is set as 1 , the optimization process will generate a result with the best performance. If $a$ is set as zero, the optimization process will generate a result with the lowest cost but this zero value is of no practical use. The performance-cost weight can be adjusted based on the real situation and budget.

\subsubsection{Optimization process by genetic algorithm}

The best solution is based on proper values of $A$ and $N$ that can minimize the cost function (4.12). In this example, $a$ is set as 0.8 , which means performance is given more weight than cost. A genetic algorithm (GA) is selected to find the best solution with the population size set as 30 , crossover rate set as 0.8 , and mutation rate set as 0.02 . The

optimization process is shown in figure 4.8. The initial number of PV panels $A$ and the 
number of wind turbines $N$ are randomly chosen. The initial result of the cost function (4.12) is shown above as $10 \times 10^{7}$, and after around 550 iterations, the result is convergent. The optimal result shows that the renewable energy farm should have $176,383 \mathrm{~m}^{2} \mathrm{PV}$ panels and 48 wind turbine generators. The optimized value is not very small when compared with conventional optimization methods which are only concerned with the performance. This is a direct result of the limitation of the second part of the cost function (4.12).

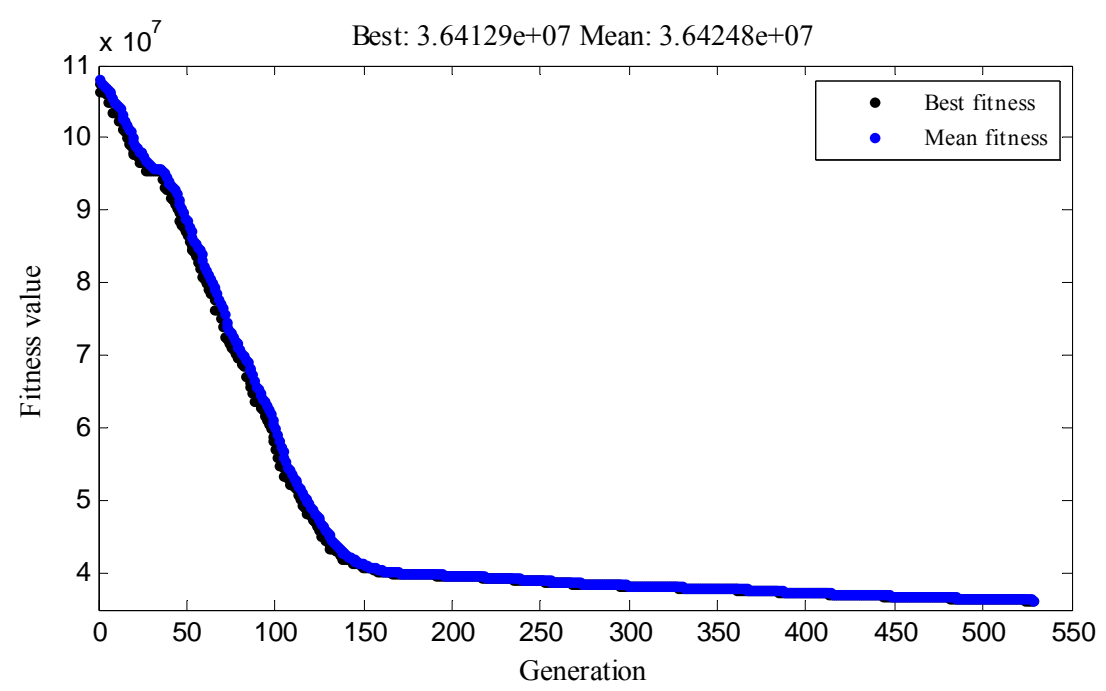

Figure 4.8 Renewable energy farm scale genetic algorithm optimization process.

\subsubsection{Optimized results analysis}

To analyze and verify the performance of the optimized result, a renewable farm with $150,000 \mathrm{~m}^{2}$ and 55 wind turbine generators (give some general info about these generators) is used for comparison. The daily energy difference between the unoptimized renewable energy farm output energy and the $20 \%$ of the Key West local load based on the collected 2005 data is shown in figure 4.9 (a). It can be seen that a renewable energy farm at this scale can't support $20 \%$ of the local load, and the power 
difference is also a negative value with the maximum power difference of approximately $-12 \times 10^{6} \mathrm{kWh}$.

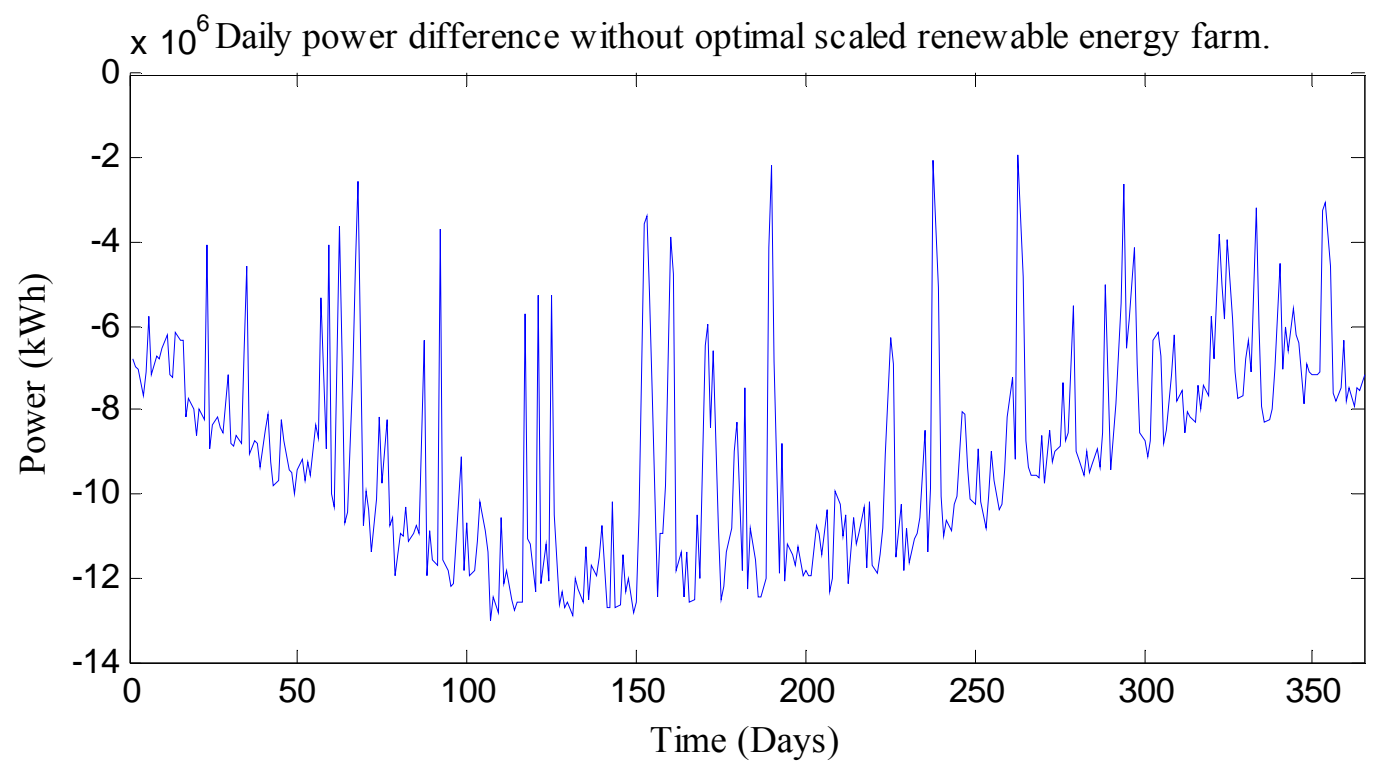

(a)

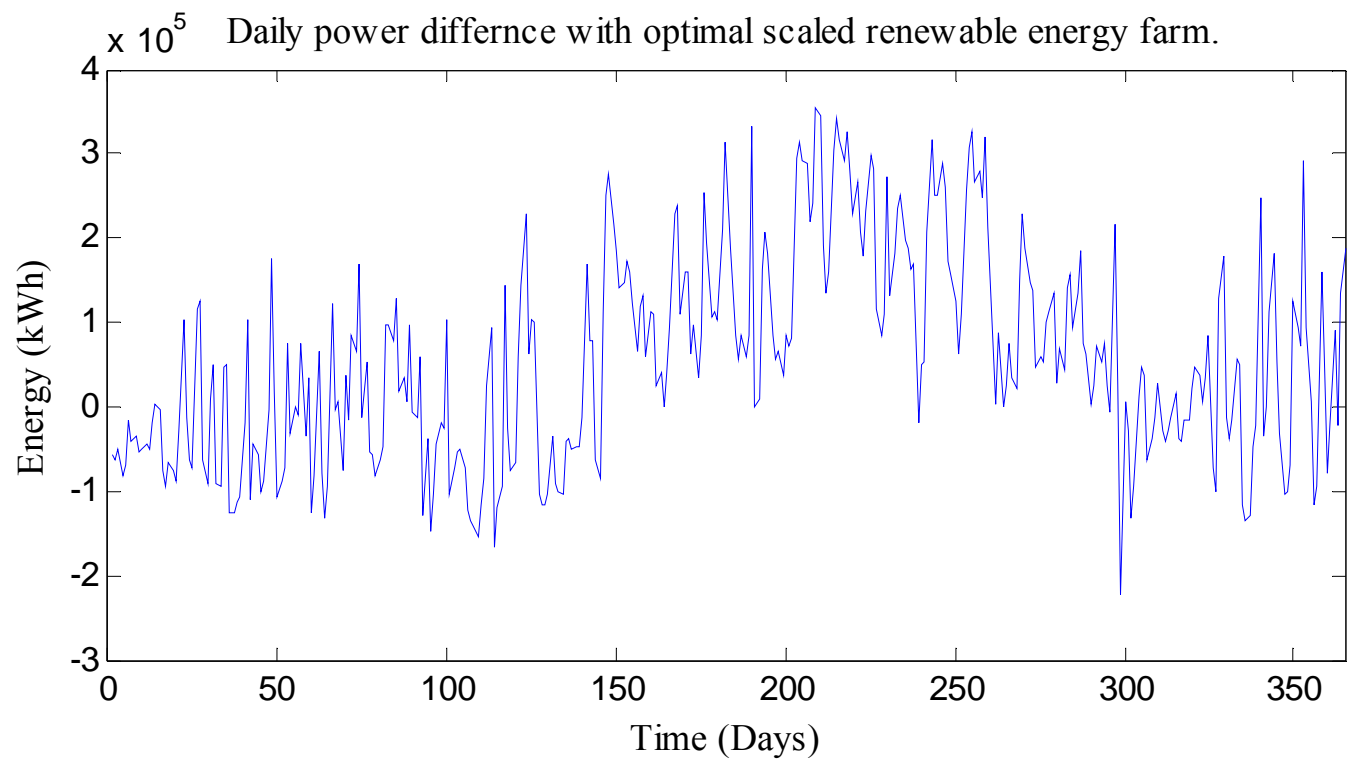

(b)

Figure 4.9 Daily energy difference between the renewable energy farm output energy and the $20 \%$ of the Key West local load: (a)without renewable energy farm scale optimization, (b) with renewable energy farm scale optimization 
Due to the fact that the power difference is always a negative value, there is no way to limit or smooth the power difference gap by using energy storage devices since there is no extra energy generated from the renewable energy farm that can be stored. What is worse, the peak to peak daily energy difference is around $10 \times 10^{6} \mathrm{kWh}$, which means the difference between the renewable energy farm daily output energy and the $20 \%$ of the local load is highly anomalous with huge variations. This makes the forecasting of the power difference very difficult. Also, the daily energy variations will impact the whole hybrid power system and cause issues such as harmonics, pollution, and instability.

The daily energy difference between the optimized renewable energy farm output energy and the $20 \%$ of the Key West local load based on the recorded 2005 data is shown in figure 4.9 (b). With the optimized renewable energy farm, the daily energy difference between the renewable energy farm and $20 \%$ of the local load is greatly suppressed. The daily energy difference varies from $-2 \times 10^{5}$ to $3 \times 10^{5}$, which is almost one tenth of the former case. Also, the daily energy differences are almost equally distributed with a mean value of $0.23 \mathrm{kWh}$.

In this situation, the energy storage system is able to reduce the daily energy gap. Also, the peak to peak daily energy difference is suppressed to around $5 \times 10^{5} \mathrm{kWh}$, almost one twentieth of the former case. This will make the forecasting much easier and improve the whole system's performance. What's more, the impacts on the utility grid from the renewable energy farm can be greatly limited. 


\subsection{Power Gaps Dispatch}

\subsubsection{Hybrid power system stability issues with high penetration of renewable}

energy sources.

From the Figure 4.10, one can observe that due to the intermittent nature of renewable energy resources, even an optimized renewable energy farm can't guarantee $20 \%$ of daily local load. Based on the simulated result, there is a rapid variation in the power generated from the renewable energy farm. This generation may impact the whole utility grid which may cause the $\mathrm{AC}$ side frequency and voltage amplitude to become unstable and introduce harmonics. Therefore, energy buffers like batteries and ultracapacitors are needed as energy storage devices to smooth the power flow and limit the impact from the renewable energy farm.

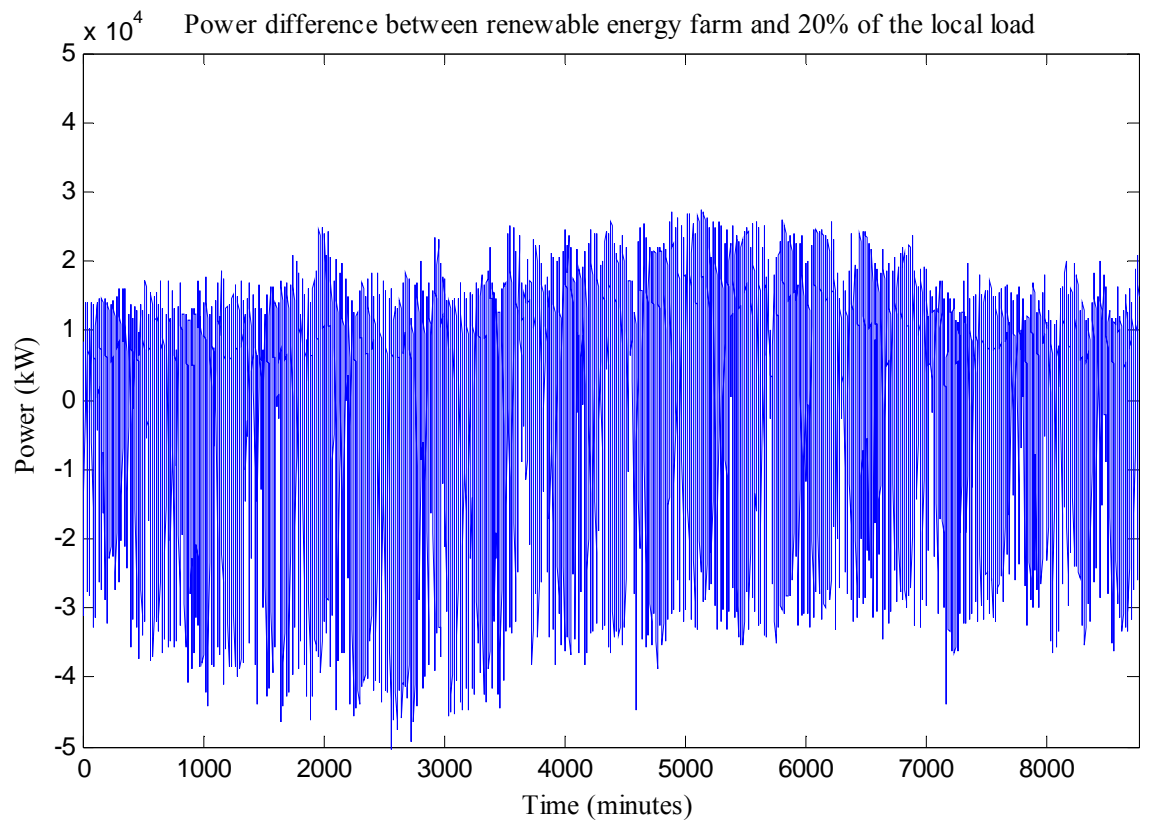

Figure 4.10 The one year minutely power difference between the renewable energy farm output and $20 \%$ of the local load 



(c)

Figure 4.11 Separation of low and high frequency power for battery and ultra-capacitor support.

\subsubsection{Impact limitation with energy storage systems}

In order to effectively partition the power gap to direct the proper energy to the battery and ultra-capacitor banks, a filter needed to be designed. After achieving the optimized renewable energy farm, the power difference between the renewable energy farm output and obtaining twenty percent of the local load, the optimal energy storage size can be designed. The power difference is passed through a low pass filter to separate the base and transient loads. These two functions are partitioned where the ultra-capacitor model is to handle $80 \%$ of the transient load profile and $20 \%$ of the base load. Conversely, the battery is set to handle $80 \%$ of the base load and only $20 \%$ of the transient load. 
The low pass filter design is progressed through a number of combinations to obtain the optimum profile. Using the MATLAB Digital Signal Processing toolbox, the filter was designed by applying frequency stop bands that correspond to a fraction of the number of hours in a year. The generalized transfer function is:

$$
F(s)=\frac{1}{\prod_{k=1}^{100}\left(1+a_{k} s\right)}
$$

where $a_{k}$ is related to factors of the cutoff frequency. The cutoff frequency in this case is normalized and operated best at $45^{\circ}$ per sample.
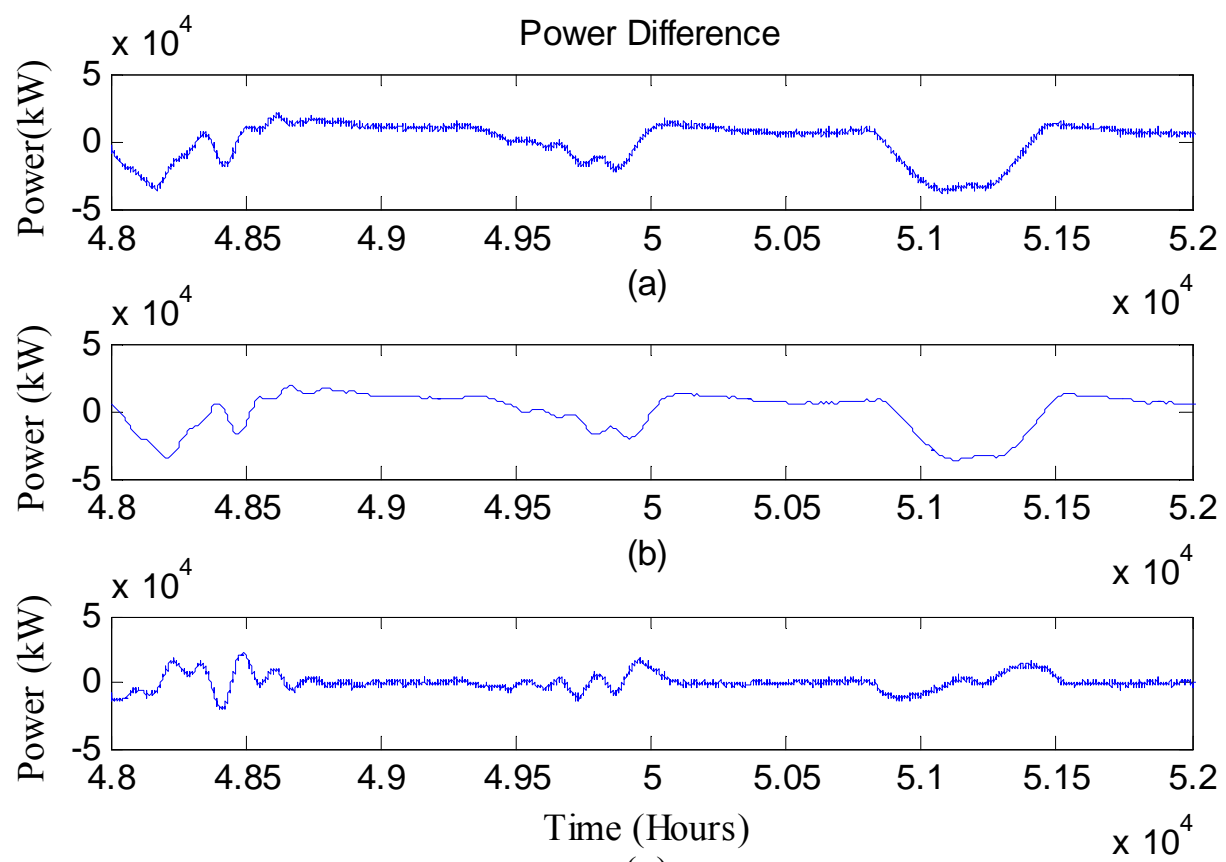

(c)

Figure 4.12 Power difference in one day period and its high frequency and low frequency parts.

The filter in (4.13) was applied to the input power difference waveform. The load profile is split to designate the high frequency portion of the load profile to be placed on the ultra-capacitors and the low frequency "base" loads to be placed on the batteries. After optimization, it was decided to further partition the LPF and HPF portions with an 
80/20 split. An advanced 100-order energy filter was implemented under the transfer function shown where $a_{\mathrm{k}}$ is related to a cutoff frequency of $125 \mathrm{mHz}$ (or 8 minutes). The output produced 2 separate plots as shown in figure 4.11 where the original waveform is shown in figure 4.11 (a). The output from the low pass filter is separated into the "Power Difference for Battery (LPF)" shown in figure 4.11(b) The remaining net energy which had not yet been compensated for was then separated into the "Power Difference for Ultra capacitor (HPF)" shown in figure 4.11(c). Through closer inspection, one can observe the transients in the power input/output primarily handled by the ultra-capacitor in figure 4.11 (c) and the smoother battery waveform in figure 4.11 (b). After applying the filter, the power difference in one day period and its high frequency and low frequency parts are shown in figure 4.12 (a), (b) and (c).

\subsection{Energy Storage System Scale Optimization}

To optimize the design of an energy storage system, the mathematic model of the energy storage system is needed. In this study, the energy storage system contains both battery and ultra-capacitor, and for cost performance concerns, lead-acid battery is chosen instead of lithium-ion battery.

\subsubsection{Lead acid battery charging/discharging mathematic model}

For practicality, the utility energy storage array is selected to use lead-acid batteries, as opposed to lithium-ion. Although lithium-ion batteries are more versatile and can withstand higher discharging and charging currents, their immense cost associated with a utility array reduces their feasibility. 
The discharging limitation is based on two major factors: empirical testing and evaluation of 3-cell lead acid batteries and practical performance expectations. Battery performance and evaluation testing is typically conducted under a constant current, but for this study it is extremely unlikely to see a battery under a constant current load. A typical load profile placed on the battery would be expected to be relatively resistive. When forecasting runtime, a resistive load is used which follows a range of currents that are inversely proportional to the operational voltage range. This progression results in a discharging current that has a mild slope, shifting $\pm 4 \%$ from the current drawn at fully charged versus fully discharged. In the discharging model, a function of the current SOC, is simply:

$$
I_{b D C \max }=-0.03 C_{B}[1-0.0008 \operatorname{SoC}(t)]
$$

The charging model is crucial as it reveals that the constant current, or linear transfer of energy, is only possible up to approximately $70 \%$ of the SOC. From a fully discharged state, constant current can be sourced to charge the battery and the energy absorbed would remain linear with respect to the available input power. However, as the battery reaches the end of its constant current stage and enters constant voltage, this phenomenon provides an indication that the electrolyte has begun to saturate. Once this saturation stage has been reached, the battery will no longer be capable of accepting energy at the same current and will continue to decrease until it has reached a fully charged state.

$$
\begin{aligned}
& I_{b C \max }= \\
& \left\{\begin{array}{cc}
0.03 C_{B}, & S O C(t) \\
C_{B}\left[0.2722 S O C^{2}(t)-56 S O C(t)+2906\right] \times 10^{-4} & S O C(t) \geq 0.7
\end{array}\right.
\end{aligned}
$$


Therefore, the battery's maximum charging and discharging power are described as equations (4.16), and (4.17). Where $V_{d c}$ is the voltage of the DC bus shown in figure 4.1.

$$
\begin{aligned}
& P b_{C \text { max }}=V_{d c} \times I_{b C \text { max }} \\
& P b_{D C \text { max }}=V_{d c} \times I_{b D C \text { max }}
\end{aligned}
$$

The battery SOC at each time step is described in equation (4.18), where $B_{c}$ is the battery capacity, $P_{b}$ is the charging/discharging power, and $T$ is the time interval.

$$
S O C(t)=\frac{B_{c} \times S O C(t-1)+P_{b} \times T}{B_{c}}
$$

\subsubsection{Ultra-capacitor model charging/discharging mathematic model}

The charging/discharging process of the ultra-capacitor is expressed in equation (4.19). If $I_{0}$ is positive, the ultra-capacitor is charging. If $I_{0}$ is negative, the ultra-capacitor is discharging. The charging and discharging power limit is shown in equations (4.20) and (4.21)

$$
\begin{aligned}
& u_{C}(t)=\sqrt{\frac{C_{0}^{2}}{4 k_{C}^{2}}+\frac{1}{k_{C}}\left(U_{C} C_{0}+U_{C}^{2} k_{C}+I_{0} t\right)}-\frac{C_{0}}{2 k_{C}} \\
& 0 \geq P c_{D C} \geq P c_{D C \max }=\frac{u_{C}^{2}}{4 R_{C 0}} \\
& 0 \leq P c_{C} \leq P c_{C \text { max }}=\frac{U_{0 \max }\left(U_{0 \max }-U_{C}\right)}{R_{C 0}}
\end{aligned}
$$

where $C_{0}$ is the initial linear capacitance, $R_{C 0}$ is the internal resistance of the ultracapacitor, and $k_{C}$ is a positive coefficient which represents the effects of the diffused layer of the ultra-capacitor. $U_{0 \max }$ is the maximum voltage of the ultra-capacitor. The ultra-capacitor capacity can be calculated based on equation (4.22).

$$
C\left(u_{C}\right)=C_{0}+k_{C} u_{C}
$$




\subsubsection{Energy storage system scale optimization cost function design}

After separating the power difference into a base component and high frequency transient component, the second cost function based on the energy storage performance and the construction cost is designed as equation (4.23).

$$
f_{2}\left(C_{c}, B_{c}\right)=\beta \sum_{d=1}^{d=365}\left(E B_{\text {penalty }}(d)+E C_{\text {penalty }}(d)\right)+(1-\beta)\left(C_{C} \times P C_{u}+C_{B} \times P B_{u}\right)
$$

where $C_{B}$ and $\mathrm{C}_{\mathrm{C}}$ are the total battery capacity and ultra-capacitor capacity, respectively. $E B_{\text {penalty }}$ and $E C_{\text {penalty }}$ are the daily penalties which can be calculated from equations (4.24) and (4.25) used to evaluate the performance of the batteries and ultracapacitors. Like $a, \beta$ is a performance-cost weight which can be adjusted based on real cases.

$$
\begin{aligned}
& P b_{\text {penally }}=\left\{\begin{array}{lc}
0 & P b_{D C \text { max }} \leq P b \leq P b_{C \text { max }} \\
\left|P b-P_{C \text { max }}\right| & P b>P b_{C \text { max }} \\
\left|P b-P_{D C \text { max }}\right| & P b<P b_{D C \text { max }}
\end{array}\right. \\
& P c_{\text {penalty }}= \begin{cases}0 & P c_{D C \text { max }} \leq P c \leq P c_{C \text { max }} \\
\left|P c-P_{s c \text { max }}\right| & P c>P c_{c \text { max }} \\
\left|P c-P_{s c \text { min }}\right| & P c<P c_{d c \text { min }}\end{cases}
\end{aligned}
$$

where the maximum charging/discharging power of battery and ultra-capacitor can be found from equations (4.16), (4.17), (4.20), and (4.21). Therefore, the daily penalty for the batteries and ultra-capacitor arrays can be calculated by equations (4.26) and (4.27), respectively.

$$
\begin{aligned}
& E B_{\text {penally }}=\int_{t=0}^{t=24} P b_{\text {penalty }}(t) d t \\
& E C_{\text {penalty }}=\int_{t=0}^{t=24} P c_{\text {penaly }}(t) d t
\end{aligned}
$$




\subsubsection{Optimization process by genetic algorithm}

The best solution is based on proper values of $C_{B}$ and $C_{C}$ that can minimize the cost function in (4.23). GA is selected to find the best solution with population size set as 20 , crossover rate set as 0.83 and mutation rate set as 0.03 . By using equation (4.23), the optimized scale of the battery and ultra-capacitor is found by using GA.

Because of the high cost of batteries and ultra-capacitors, the performance-cost weight $\beta$ is set as 0.7 . After around 150 iterations, the result is convergent. The optimal result shows that the energy storage system should contain $358,03012 \mathrm{~V}$ lead-acid batteries where 26 are connected in a series array to meet the DC bus voltage and 13,805 arrays are placed in parallel to meet current capacity requirements. The energy storage system also needs a capacitor bank with the total of 191,303 ultra-capacitors connecting in parallel.

\subsubsection{Optimized results analysis}

With the optimized energy storage system, the power difference between the renewable energy farm output power and $20 \%$ of the local load is greatly reduced compared to the system without the optimized energy storage system. The minute power difference between the renewable energy farm output and the twenty percent of the local load is shown in figure 4.13. Compared with figure 4.9(b), the power gap is much smaller when the system has the optimal scaled energy storage system. Therefore, the optimized energy storage system can help the renewable energy farm smoothly transfer power to the utility grid to support the local load. 


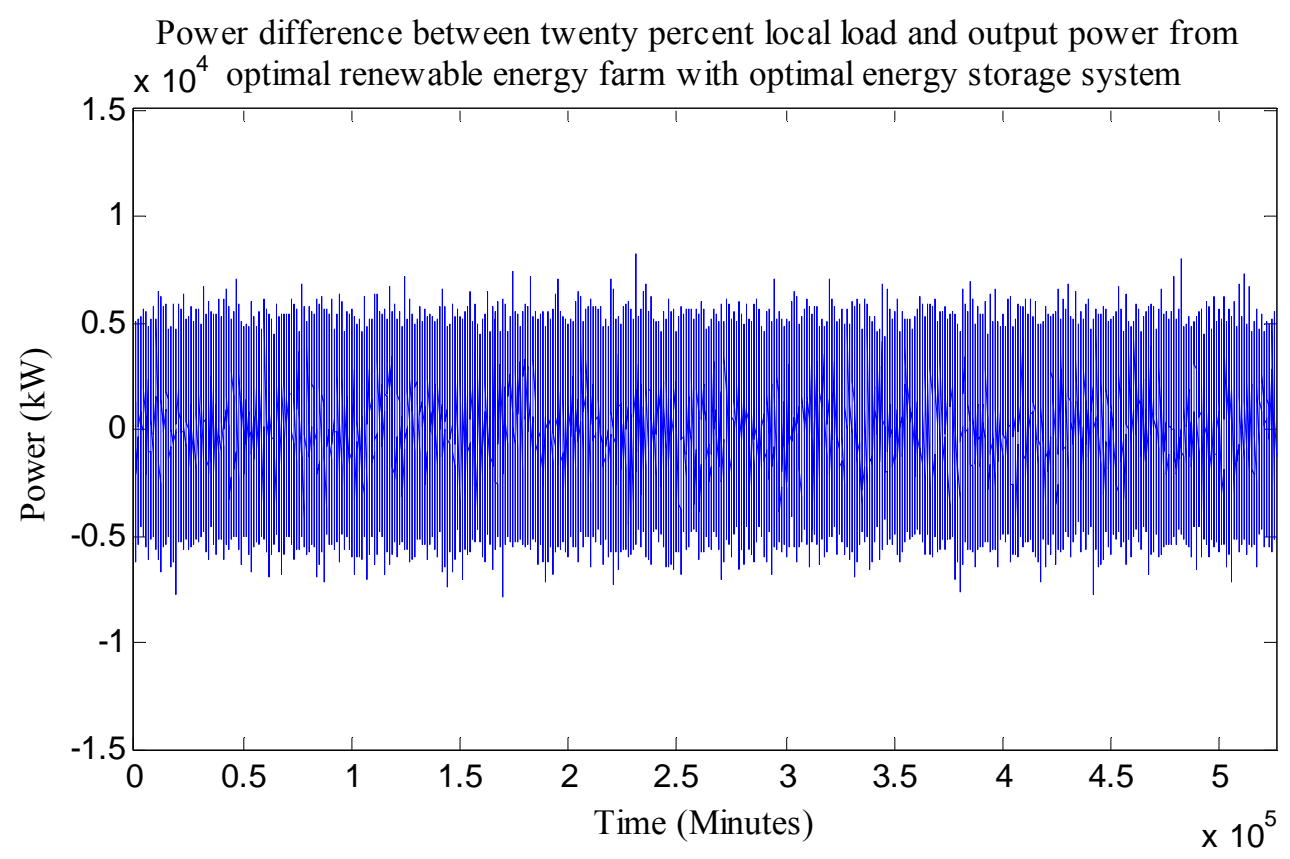

Figure 4.13 The power difference between the renewable energy farm output and $20 \%$ of the local load with optimized energy storage system.

\subsection{Conclusion}

In this chapter, an optimal sizing method for a renewable energy farm and energy storage devices in a hybrid power system is proposed. A genetic algorithm is used to find the optimal solutions for both renewable farm and energy storage devices. With the optimized scale of solar panels and wind turbine generators, the renewable energy farm can supply around twenty percent of the local load with less fluctuation and cost. The energy storage system with the optimized battery and ultra-capacitor sizes has the ability to smooth the energy flow from the renewable energy farm to the local load, which will greatly limit the impact on the utility grid from the renewable energy farm caused by uncertain factors such as the sudden change of solar irradiance and wind speed. A case study of the Key West, FL power system is performed. Simulation results are presented to validate the advantages of the proposed optimal sizing method. 
5. Development of High Accuracy Lithium-ion Battery Modeling with the Consideration of Thermal Effect

\subsection{Introduction}

Batteries used as energy storage devices will play an important role in future power systems due to the growing popularity of micro grids (MGs) [113]-[114] and plugin electric vehicles (PEVs). The performance of the MGs and PEVs strongly relies on their battery bank management systems, which always consists of multiple cells connected together in series and in parallel. The energy conversion efficiency of the battery bank system is influenced by a single battery cell's state of charge (SOC) and temperature variations. Thermal and SOC imbalances increase the risk of catastrophic faults in the battery bank system. The recent Boeing 787 Dreamliner's lithium-ion battery faults in Japan and Boston have illustrated the importance of developing reliable and efficient battery management systems in high energy density battery systems [13]. The specific characteristics and needs of the smart grid and EVs, such as deep charge/discharge protection and accurate SOC and state of health (SOH) estimation, intensify the need for a more efficient battery management system [115]-[116].

PEV's have seen an expansive growth over the past several years and with their growth in popularity has come the demand for more reliable and efficient battery management systems. PEVs rely heavily on the capability of their battery management systems to monitor and manage their SOC and temperature variations in order to increase the capacity, reliability, and lifetime of the individual cells that make up their battery 
system. Imbalances and variations between individual cells often occur throughout the battery banks, and these variations can range from discharge rates and internal impedances to energy densities. Imbalances in cell temperatures and SOC can lead to individual cells being overcharged, over discharged, and overheated. These failures can propagate throughout the entire battery system and severely diminish the efficiency and reliability of the system. The optimal operating environment of lithium-ion batteries ranges from $30^{\circ} \mathrm{C}$ to $40^{\circ} \mathrm{C}$, and every $10^{\circ} \mathrm{C}$ increase in the temperature halves the life of the battery [117].

This chapter presents a battery management system that is able to self-heal from faults in order to avoid SOC and thermal imbalances using cell switching circuits. Both internal and external temperatures are taken into account in the design to provide full protection from all possible thermal imbalances. The SOC of individual cell banks in their corresponding modules is also taken into account in order to protect from variations in SOC within the battery system.

The proposed lithium-ion battery model introduced thermal effects into the equivalent circuit of the lithium-ion battery model and developed a battery management system that can truly self-heal from any variations in individual cell characteristics. A novel fuzzy logic based battery bank management system is presented. The battery bank management system will have self-healing capabilities to protect itself from SOC and thermal imbalances. Furthermore, an accurate lithium-ion battery thermal model is presented to emulate the lithium-ion battery state during the charging and discharging process. The proposed design is validated by using MATLAB Simulink to present a battery management system that balances the SOC and temperature of 5 battery modules 
where each module consists of a $6 \times 3$ configuration of lithium-ion cells connected in series and parallel. The proposed battery bank power and thermal management system can effectively regulate the temperature and balance the SOC's of the cells in the battery bank during the charging and discharging process, which will largely increase the safety of the whole system.

The chapter is organized as follows: Section 5.2 presents advantages of lithiumion batteries and their application. Section 5.3 explains the importance of lithium-ion battery modeling and the energy management system. Section 5.4 presents the high accuracy equivalent circuit model, along with the detail of SOC and thermal emulations for the lithium-ion battery. Section 5.5 discusses the fuzzy logic based battery banks energy management system and its switching circuit topology. Section 5.6 describes the method of real-time battery bank operation in a hybrid power system with droop control. Finally, section 5.7 provides the conclusion and discussions on this topic.

\subsection{Advantages of Lithium-ion Batteries and Their Application}

There are many advantages in using lithium-ion batteries. These advantages include:

- High energy density: Their much greater energy density is one of the chief advantages of a lithium-ion battery or cell. With electronic equipment such as mobile phones needing to operate longer between charges while still consuming power, there is always a need for batteries with a much higher energy density. In addition, there are many power applications ranging from power tools to electric 
vehicles. The much higher power density offered by lithium-ion batteries is a distinct advantage.

- Self-discharge: One issue with batteries and individual cells is that they lose their charge over time. This self-discharging effect can be a major issue. One advantage of lithium-ion cells is that their rate of self-discharge is much lower than that of other rechargeable cells such as Ni-Cad and NiMH forms.

- No requirement for priming: Some rechargeable cells need to be primed when they receive their first charge. There is no requirement for this with lithium-ion cells and batteries.

- Low maintenance: One major lithium-ion battery advantage is that they do not require constant maintenance to ensure their performance. Ni-Cad cells require a periodic discharge to ensure that they do not exhibit the memory effect. (Define this memory effect and why it is detrimental) As this does not affect lithium-ion cells, this process or other similar maintenance procedures are not required.

- Variety of types available: There are several types of lithium-ion cells available. This advantage allows for particular types or chemistries of lithium-ion cells with specific characteristics to be chosen for particular applications. Some forms of lithium-ion batteries provide a high current density and are ideal for consumer mobile electronic equipment. Others are able to provide much higher current levels and are ideal for power tools and electric vehicles. This variation allows power systems engineers to be more selective with respect to the battery's application. 


\subsection{Importance of Lithium-ion battery modeling and energy management systems}

The battery energy management system is an important element to be utilized in making the modern hybrid power system and PEVs safe, reliable, and efficient. The battery management system should not only control the operational conditions of the battery to prolong its life and guarantee its safety, but also provide accurate estimation of the SOC, temperature, and SOH for the battery banks in the smart grid and PEVs. To fulfill these tasks, a battery management system has several features to control and monitor the operational state of the battery at different battery cells, battery modules, and battery pack levels is needed [14].

\subsubsection{Over charge/discharge impact on lithium-ion batteries}

The lithium-ion battery is a voltage-limiting device that is similar to the lead acid system. The difference lies in a higher voltage per cell, tighter voltage tolerance, and the absence of trickle or float charge at full charge. While lead acid offers some flexibility in terms of voltage cut-off, manufacturers of lithium-ion cells are very strict on the appropriate configuration because lithium-ions cannot accept overcharge. The lithium-ion produces a "clean" system and only takes what it can absorb. Anything extra causes stress. If overheated or overcharged, lithium-ion batteries may suffer thermal runaway and cell rupture (details explained in 5.3.2). In extreme cases this can lead to combustion.

Overcharging Lithium-ion: Lithium-ion batteries operate safely within the designated operating voltages; however, the battery becomes unstable if inadvertently charged to a higher than specified voltage. Prolonged charging above $4.30 \mathrm{~V}$ forms plating of metallic lithium on the anode, while the cathode material becomes an oxidizing 
agent, loses stability, and produces carbon dioxide (CO2). The cell pressure rises, and if charging is allowed to continue, the current interrupt device responsible for cell safety disconnects the current at $1,380 \mathrm{kPa}(200 \mathrm{psi})$.

Should the pressure rise further, a safety membrane bursts open at $3,450 \mathrm{kPa}$ (500psi) and the cell might eventually vent with flame. The thermal runaway moves lower when the battery is fully charged; for lithium-cobalt this threshold is between 130 $150 \mathrm{C}^{\circ} \mathrm{C}\left(266-302^{\circ} \mathrm{F}\right)$, nickel-manganese-cobalt $(\mathrm{NMC})$ is $170-180^{\circ} \mathrm{C}\left(338-356^{\circ} \mathrm{F}\right)$, and manganese is $250^{\circ} \mathrm{C}\left(482^{\circ} \mathrm{F}\right)$. Lithium-phosphate has better temperature stabilities than manganese.

Over-discharging Lithium-ion: Lithium-ion should never be discharged too low, and there are several safeguards to prevent this from happening. The equipment cuts off when the battery discharges to about $3.0 \mathrm{~V} /$ cell, stopping the current flow. If the discharge continues to about $2.70 \mathrm{~V} /$ cell or lower, the battery's protection circuit puts the battery into a sleep mode. This renders the pack unserviceable and a recharge with most chargers is not possible. To prevent a battery from falling asleep, a partial charge is applied before a long storage period. Also, if a lithium-ion cell has stayed at or below 1.5 V for more than a week, it should not be charged anymore because copper shunts may have formed inside the cells that can lead to a partial or total electrical short. If recharged, the cells might become unstable, causing excessive heat or showing other anomalies. Lithium-ion battery banks that have been under stress are more sensitive to mechanical abuse, such as vibration, dropping and exposure to heat [118]. 


\subsubsection{Over heat impact to lithium-ion battery}

Temperature is an important factor in the operation of a battery. In addition to the safety issue that is defined by the temperature range, the efficiency of the battery is also affected by the ambient temperature because of degradation of its capacity and an increase in internal resistance. Meanwhile, the consequence of heat evolution depends on the environment of the cells. When the cells are in an environment where heat can be evacuated, the reactions will stabilize and cells will progressively cool down. This corresponds, for example, to batteries with cooling systems, or small batteries evacuating the heat through their external casing. In contrast, when the heat cannot be evacuated (such as in a confined environment, or even worse, in a heated environment), the battery temperature will increase, and will reach a status where new reactions can start, generating even more heat. This mechanism is called "thermal run-away".

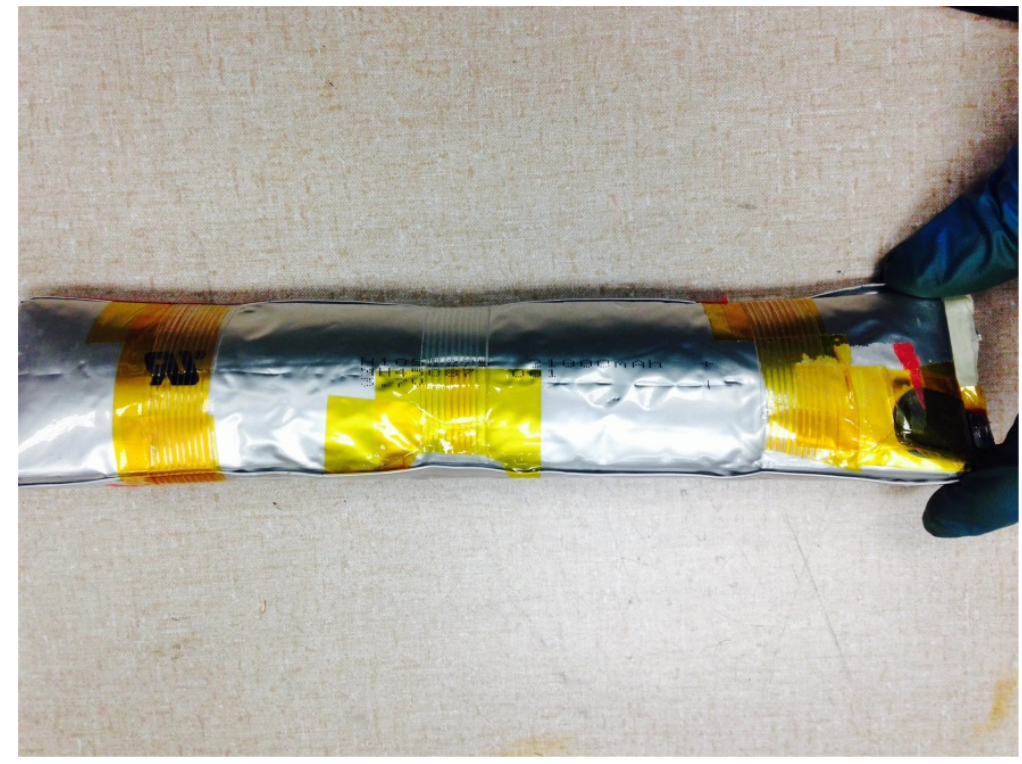

Figure 5.1 Over charged lithium-ion battery cell 


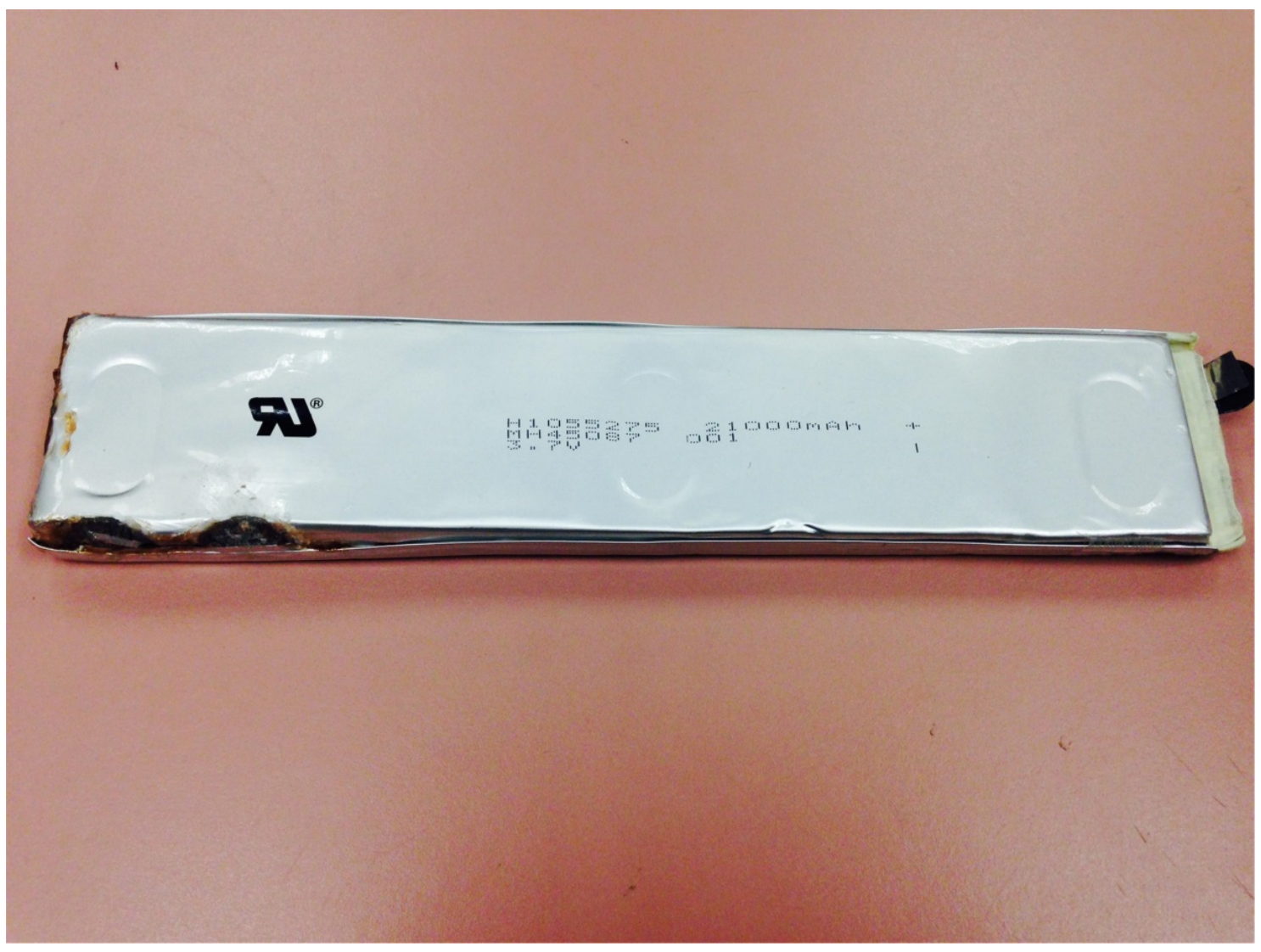

Figure 5.2 Over heat impact to lithium-ion battery cell.

Several reactions involving the separator, electrolyte and positive terminal could be ignited with temperature increases. Consequently, the heating rate accelerates from less than $1^{\circ} \mathrm{C} /$ minute to more than $100^{\circ} \mathrm{C} /$ minute. Without appropriate design to limit the run-away (such as venting, insulating layers, etc...), it can lead to a violent emission of gas and flames [119]. Therefore, the battery management system needs to have the ability to control the temperature of the battery and keep it at the optimal point under different operating conditions. The need to dissipate the heat produced by the battery cells due to electrochemical reactions will be more serious when several cells are compacted in a battery pack. Thermal management uses heat-transfer analysis to determine the 
distribution of heat inside the battery pack and embed channels to remove the heat using air or a liquid, if necessary.

\subsection{High Accuracy Lithium-ion Battery Model}

Numerous lithium-ion battery models have been developed in recent years to characterize their dynamic characteristics during charging and discharging processes. The choice of model corresponds to a tradeoff between computational complexity and the ability to fit real experimental data. A complex equivalent circuit may perfectly fit the experimental data but require tremendous amount of computational time and memory to solve detailed partial differential equations of the battery model. Complex models are limited in their application for embedded control and real-time applications. The equivalent circuit model used in this design is given in figure 5.3(a), which is a commonly used representation of a lithium-ion cell that simplifies their numerical

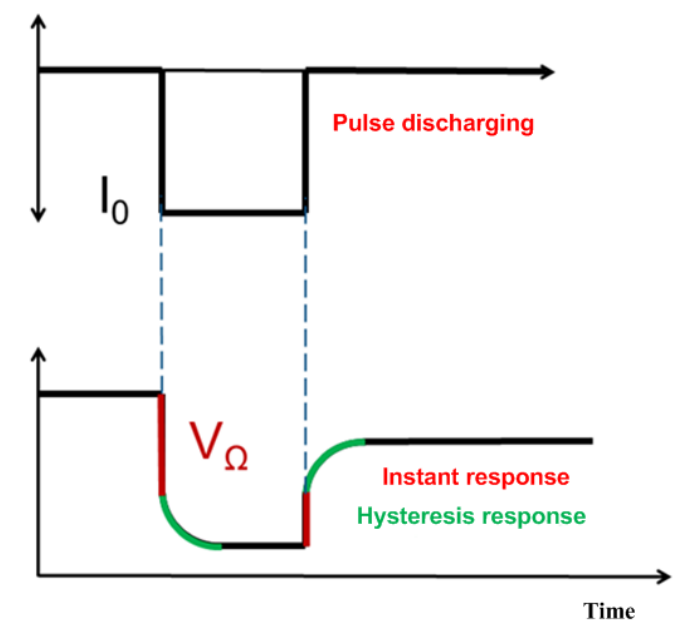

(a)

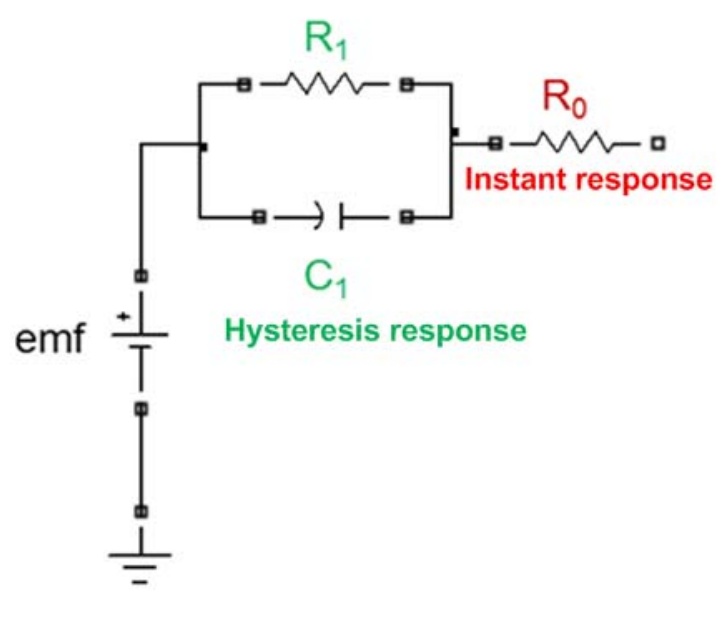

(b)

Figure 5.3 (a) lithium-ion battery pulse discharging response (b) lithium-ion battery equivalent circuit 
analysis while sufficiently accounting for all the dynamic characteristics of the cell. The series resistance simulates the short term instant transient response while the parallel resistance and capacitance simulate the long term hysteresis response. To improve the accuracy, more hysteresis RC blocks can be added to the model, which increases the model's complexity. For many problems of industrial relevance, one single hysteresis RC block is adequate. In this chapter, a single hysteresis RC block battery model is used. Therefore, four elements; $E_{m}, R_{0}, R_{l}$ and $C_{l}$ are needed to define the equivalent circuit of a lithium-ion battery cell.

This model takes into account all of the electrochemical processes occurring inside the lithium-ion cell and their relation to their equivalent circuit elements. Past experiments show that the equivalent circuit elements are dependent on both the SOC and temperature of the individual cell, specifically:

$$
\begin{gathered}
R_{0}=R_{0}(S O C, T) \\
R_{1}=R_{1}(S O C, T) \\
C_{1}=C_{1}(S O C, T) \\
E_{m}=E_{m}(S O C, T)
\end{gathered}
$$

These four circuit elements are defined through lookup tables that correspond to specific SOC's and temperatures. Those four tables are established by using Matlab numerical analysis [120]. The estimation tool in Simulink, along with the lookup tables allows for accurate estimation of these parameters. This parameter estimation is used in conjunction with the methods outlined in [115] to develop a battery management system that balances the SOC and temperature of its battery banks. 


\subsubsection{Lithium-ion battery SOC estimation}

The SOC is an indicator that represents the available charge stored in the battery compared to the full capacity charge. Accurate modeling, estimation, and management of this parameter will protect the battery from malfunctions resulting from overvoltage, unsafe charging rates, and deep discharges that endanger the integrity of the battery. The SOC of an individual cell is calculated by using equation (5.5).

$$
S O C=1-\frac{Q_{e}}{C_{Q}}
$$

Where $Q_{e}$ is extractable charge and $C_{Q}$ is the total capacity of the cell. Below is the equation (5.6) for extractable charge $Q_{e}$ of an individual cell along with the total capacity of the cell which is dependent on current and temperature.

$$
\begin{aligned}
& Q_{e}(t)=\int_{0}^{t} I_{m}(\tau) d \tau \\
& C_{Q}=C_{Q}(I, T)
\end{aligned}
$$

\subsubsection{Lithium-ion battery heat estimation}

The temperature of individual lithium-ion cells greatly limits their performance. Therefore, an accurate thermal model is essential to optimize the utilization of these cells in battery banks. The inner cell temperature is assumed to be uniform, and viewed as the average temperature for the single cell. The temperature of a single cell can be updated by using the heat equation (5.8) of a homogeneous body exchanging heat with the environment.

$$
C_{T} \frac{d T}{d t}=-\frac{T-T_{a}}{R_{T}}+P_{s}
$$


After the Laplace transformation, the transfer function can be expressed as equation (5.9).

$$
T(s)=\frac{P_{s} R_{T}+T_{a}}{1+R_{T} C_{T} s}
$$

where

$C_{T} \quad$ is the heat capacitance $\left(\mathrm{Jm}^{-3} \mathrm{~K}^{-1}\right)$

$P_{s} \quad$ is the power dissipated inside the cell(W)

$R_{T} \quad$ is the convection resistance $\left(\mathrm{Wm}^{-2} \mathrm{~K}^{-1}\right)$

$T \quad$ is the cell inner temperature $\left({ }^{\circ} \mathrm{C}\right)$

$T_{a} \quad$ is the ambient temperature $\left({ }^{\circ} \mathrm{C}\right)$

The power dissipated inside the cell can be calculated by using equation (5.10).

$$
P_{s}=I^{2}\left(R_{0}+R_{1}\right)
$$

\subsubsection{Real time lithium-ion battery SOC and thermal monitoring}

At each initial period, the inner temperature of the batteries should be the same as the ambient temperature $T_{a}$. Therefore, by measuring the terminal voltage $E_{m}$, the SOC of a certain cell can be found by using equation (5.3). After this estimation the SOC, $R_{0}, R_{l}$, and $C_{l}$ can be found by using equations (5.1), (5.2), and (5.3).

Upon initialization, the SOCs and temperatures of cells can be updated by measuring the current flowing through the particular cell. This measurement results in acquiring the terminal voltage $E_{m}$, which allows the SOC to be estimated. The parameter estimation table then estimates the appropriate values of resistances and capacitance. All 
parameters are then updated by measuring the current flow through the battery. The flow chart of the real time SOC and thermal monitoring algorithm is shown in figure 5.4.

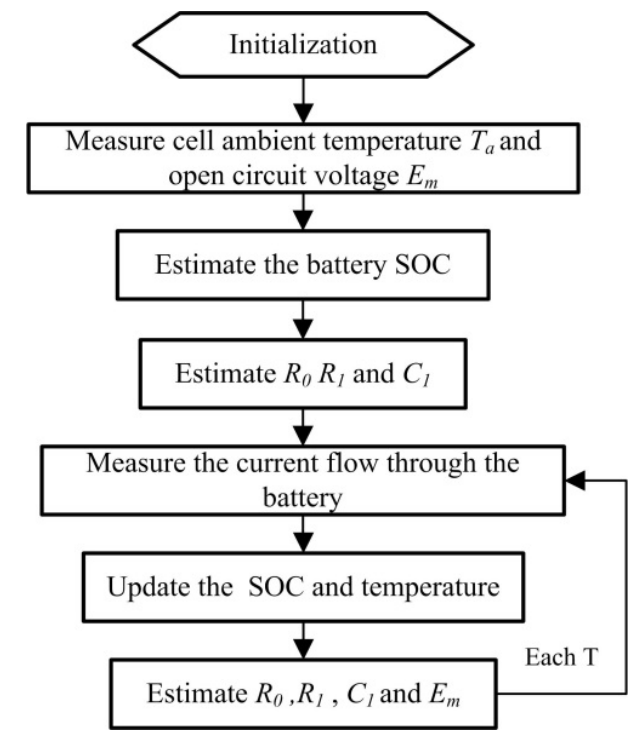

Figure 5.4 Flow chart of the real time SOC and thermal monitoring

\subsection{Battery Bank Energy Management with SOC and Temperature Self- Balancing Function}

For industrial applications, a single lithium-ion battery cell is seldom utilized because of the terminal voltage and available current limitations. Therefore, lithium-ion batteries are always grouped together as a battery bank through several of the cells in series and/or in parallel to increase the total terminal voltage and power.

\subsubsection{Importance of SOC and heat balancing}

The example of one lithium-ion battery bank is shown in figure 5.5. It contains several cells connected in series and parallel. Cell state imbalance is commonly present in traditional multi-cell battery banks, and oftentimes, the same brand of lithium-ion batteries from the same manufacturer will still have minor differences in characteristics 
such as inner resistances and capacities. Without proper control and regulation, those tiny difference may accumulate and worsen the imbalance, possibly destroying the whole battery bank. As shown in figure 5.1 and figure 5.2., without proper control, a certain cell will over heat or over charge/discharge which may ruin the whole battery bank. Even worse, the whole hybrid power system may be influenced, which may cause serious issues such as system instability, system lifetime degradation, and so on. Therefore, individual lithium-ion battery cells in a battery bank need to be carefully monitored and balanced to increase the reliability of the battery bank.

\subsubsection{Fuzzy logic battery bank energy management algorithm}

Each module contains its own management system that controls the SOC and



Figure 5.5 Lithium-ion battery bank 
thermal imbalances among its 6 banks. The switching control is derived directly from [115]. The SOC of each bank is first calculated by equation (5.11).

$$
\operatorname{SOC}_{b}=\frac{1}{n} \sum_{i=1}^{n} S O C_{i}
$$

$S O C_{i}$ is the SOC of the $i^{\text {th }}$ cell in the bank. The balancing of the banks continues with the calculation of the number of banks to be disconnected to balance SOCs and temperatures of each battery bank within the module, $q_{n b}$ :

$$
q_{n b}=f\left(\Delta S O C_{b}, \Delta T_{b}\right)
$$

Where $f(\triangle S O C b, \triangle \mathrm{T} \mathrm{b})$ is the function that determines how many banks in the module are needed to be disconnected to balance their SOC and temperature. $\triangle S O C_{b}$ is the difference between the maximum and minimum bank SOCs of the module, and $\Delta T_{b}$ is the difference between the maximum and minimum bank temperatures of the module.

Since the battery system is highly nonlinear, and the number of banks that need to be disconnected from the module has no direct relationship with $\triangle S O C_{b}$ and $\triangle T_{b}$, fuzzy logic is applied to define the function of $f\left(\triangle S O C_{b}, \triangle \mathrm{T} \mathrm{b}\right)$.

Fuzzy logic is a form of many-valued logic which deals with reasoning that is approximate rather than fixed and exact. Compared to traditional binary sets (where variables may take on true or false values), fuzzy logic variables may have a truth value that ranges in degree between 0 and 1. Fuzzy logic has been extended to handle the concept of partial truth, where the truth value may range between completely true and completely false. Furthermore, when linguistic variables are used, these degrees may be managed by specific functions. Linguistic variables are the input or output variables of 
the system whose values are words or sentences from a natural language, instead of numerical values. A linguistic variable is generally decomposed into a set of linguistic terms.

The term "fuzzy logic" was introduced with the 1965 proposal of fuzzy set theory by Lotfi A. Zadeh. Fuzzy logic has been applied to many fields, from control theory to artificial intelligence. Fuzzy logic had, however, been studied since the 1920s, as infinitevalued logic — notably by Łukasiewicz and Tarski.

Fuzzy control is based on the experience of the user about the system behavior rather than modeling the system under control mathematically like in linear control theory. This makes fuzzy control a powerful control technique especially with non-linear systems in which it is difficult to derive an accurate approximated mathematical model of the system and expect its behavior. Fuzzy control is a rule-based control technique that is approached by linguistic fuzzy rules, which describe the output desired out of the system under different operating conditions. Fuzzy rules are in the form of if-then rules that the proficient should design such that they cover all the conditions the system is expected to go through.

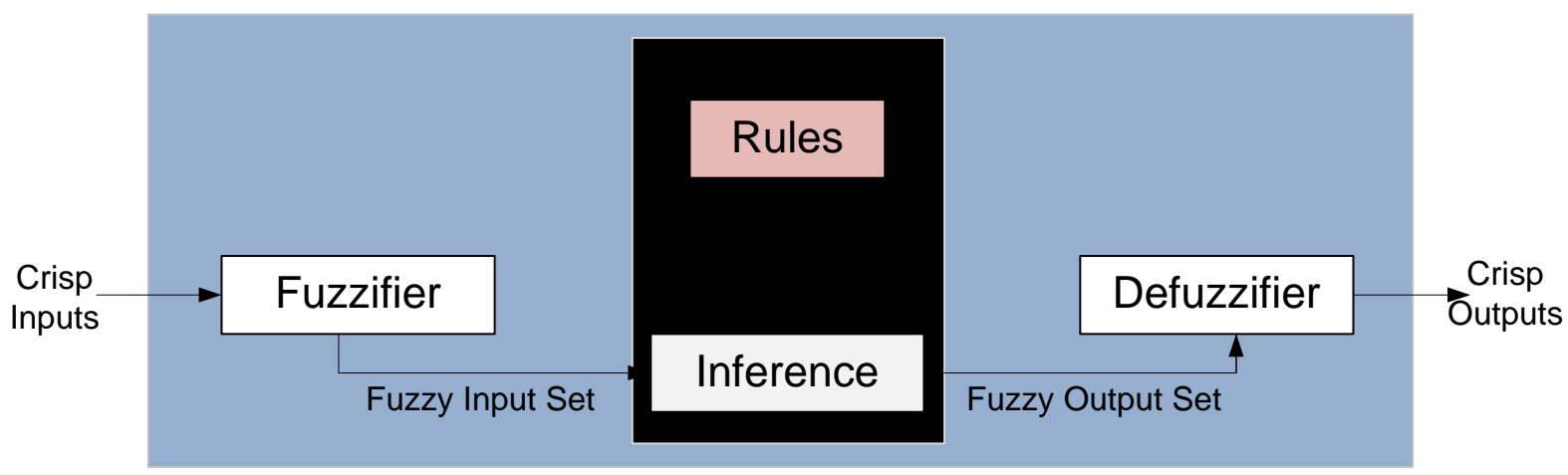

Figure 5.6 Fuzzy logic control steps 
The architecture of a fuzzy controller is shown in figure 5.6. A fuzzy logic controller is designed through four major steps: fuzzification, rules, inference mechanism, and defuzzification.

The basic process of fuzzy logic is shown in Table 5.1: Firstly, a crisp set of input data are gathered and converted to a fuzzy set using fuzzy linguistic variables, fuzzy linguistic terms and membership functions. This step is known as fuzzification. Afterwards, an inference is made based on a set of rules. Lastly the resulting fuzzy output is mapped to a crisp output using the membership functions, in the defuzzification step.

Table 5.1 Fuzzy Logic Algorithm Basic Process

1. Define the linguistic variable and terms(initialization)

2. Construct the membership functions(initialization)

3. Construct the rule base (initialization)

4. Convert crisp input data to fuzzy values using the membership functions (fuzzification)

5. Evaluate the rules in the rule base (inference)

6. Combine the results of each rule (inference)

7. Converter the output data to a non-fuzzy values (defuzzification)

The two inputs of the fuzzy logic controller are $\triangle S O C_{b}$ and $\triangle T_{b}$, and the output of the fuzzy logic controller is $q_{n b}$. Mamdani-type fuzzifaction and defuzzifaction is applied for the fuzzy logic function. The $\triangle S O C_{b}$ is described as "very small", "small", "normal", "big" and "very big", which show the SOC difference between different battery banks by using linguistic variables. The $\Delta T_{b}$ is described as "very low", "low", "normal", "high", and "very high", which show the temperature difference between different battery banks in linguistic variables. The output of the fuzzy logic controller $q_{n b}$ is described by "balanced", "tiny imbalance", "medium imbalance", "imbalance”, "urgent”. For example, 
at a certain time the $\triangle S O C_{b}$ is very small and the $\triangle T_{b}$ is very low, therefore, this module is in a balanced state with respect to SOC and temperature. Also, the output of the fuzzy controller is balanced, which means no battery within this module needs to be disconnected for balancing purposes. At another moment, if the $\triangle S O C_{b}$ is very big and the $\Delta T_{b}$ is very high, then the fuzzy logic controller's output may be "urgent", and after defuzzifaction, the controller may disconnect battery banks to regulate the system SOC and temperature. Because each module has only 6 battery banks, the maximum $q_{n b}$ is set as 3 in order to keep enough battery banks operating in the module. The membership functions of the fuzzy logic controller and the rule surface are shown in figure 5.7. (a), (b), (c), and (d).

Several battery modules can be connected together in series to act as an energy storage device with a far higher energy capacity. In this configuration, the energy storage device is suitable for utilization in PEVs and MGs. The thermal and SOC balancing method proposed in this chapter can also be used to regulate the modules in a similar fashion. The only differences are the inputs to the fuzzy function which become the average SOCs and temperatures of each module instead of each battery bank.

\subsubsection{Battery bank SOC and temperature self-balancing circuit}

The design of the battery SOC and temperature self-balancing circuit serves to monitor and manage the SOC and thermal imbalances that occur in a lithium-ion battery module. The example module contains $m$ battery banks, each with $n$ cells, thus, a $m \times n$ lithium-ion cell configuration is implemented in the bank. The switching topology used in each module connects all 6 banks in series and each of the three cells within the 
module in parallel. This cell switching topology serves to disconnect specific banks of cells during discharging or charging cycles so that the other banks can balance any SOC

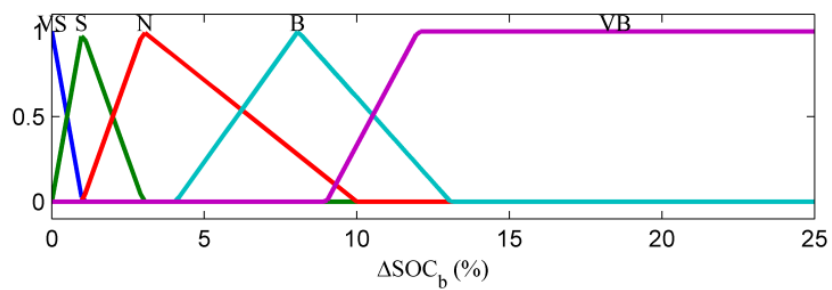

(a)

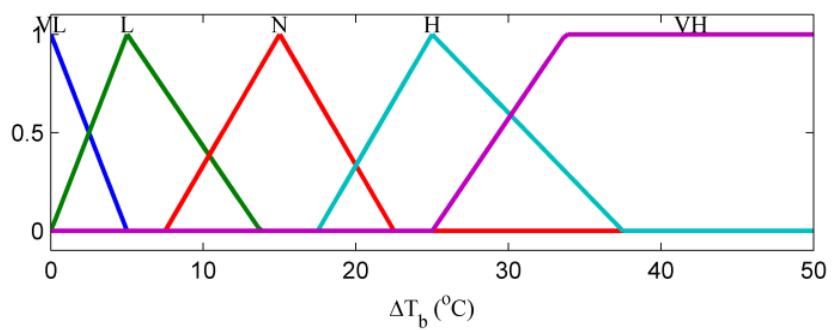

(b)

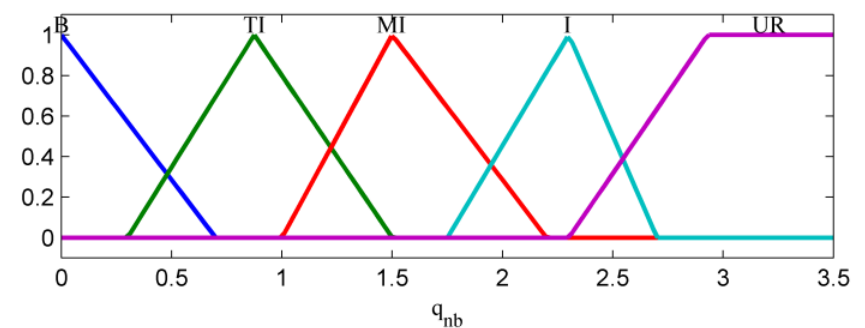

(c)



(d)

Figure 5.7 (a) $\triangle S O C_{b}$ membership function. (b) $\triangle T_{b}$ membership function. (c) $q_{n b}$ membership function. (d) Fuzzy logic controller rule surface. 
or temperature variations. The lithium-ion battery module SOC and temperature selfbalancing circuit is shown in figure 5.8.

For example, at a certain moment, cell C21 temperature is higher than the rest of the cells in the bank, which increases the average temperature of bank 2. If the temperature difference exceeds a certain limit, switches $S_{21}$ to $S_{2 n}$ should turn off and switch $S_{2}$ should be turned on. In this way, bank 2 is isolated to cool down.

Another example, at a certain moment, the SOC of cell $C_{m n}$ is low. Then, the

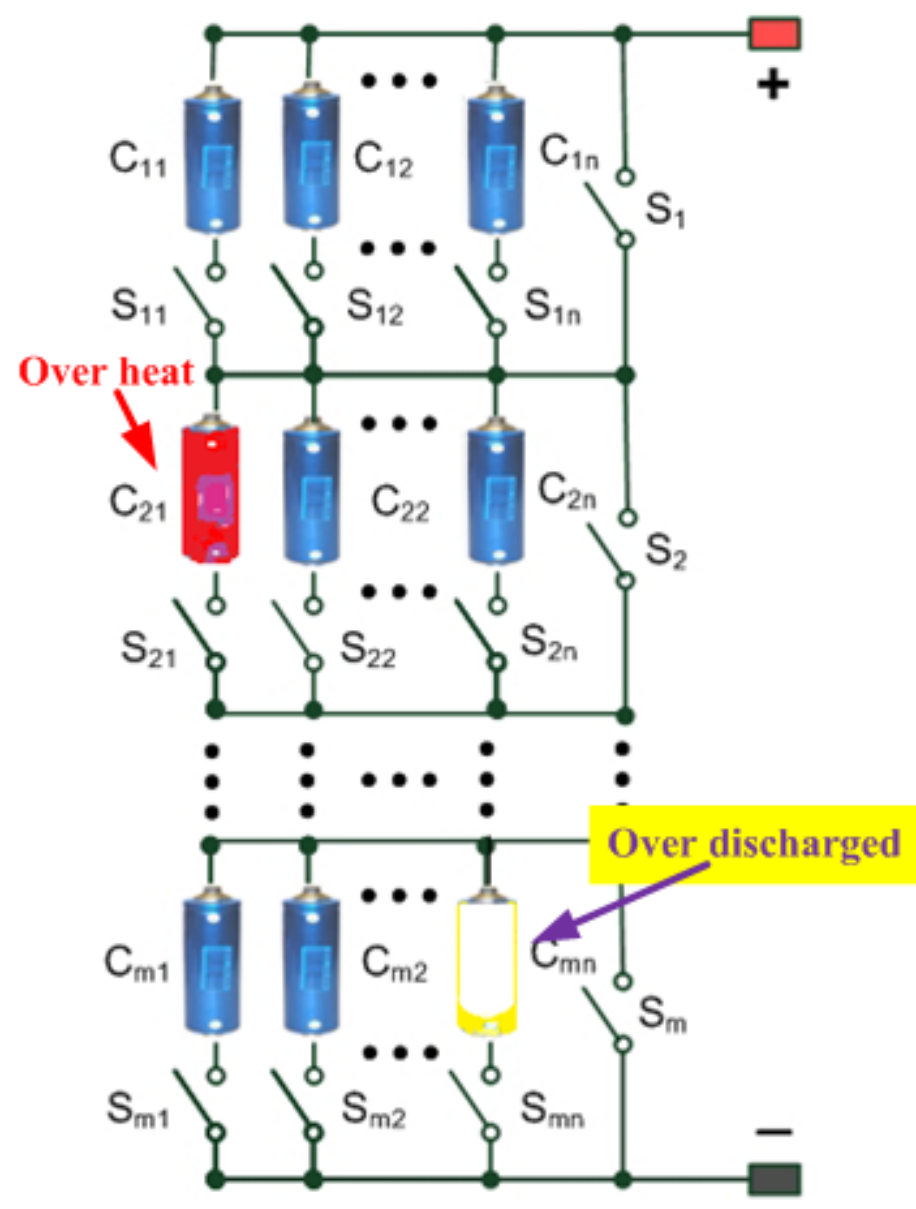

Figure 5.8 Lithium-ion battery bank SOC and temperature self-balancing circuit 
average SOC of bank $\mathrm{m}$ is lower than the rest of the banks. If the battery module is in a discharging situation, after exceeding the SOC difference limit, bank $\mathrm{m}$ should be isolated from the rest of the banks in the battery module. To fulfill this function, the selfbalancing circuit will turn off switches $S_{m n}$. After isolation from the battery banks, cell $\mathrm{C}_{\mathrm{mn}}$ will be disconnect. It will reconnect back to the bank once the SOC differences between other banks are within the limit, or the whole module is changed to charging mode. After reconnection, their SOCs will be automatically balanced since cell $C_{m l}$ to $C_{m n}$ are connected in parallel.

\subsubsection{Simulation and experimental verification}

The battery management system's operation is simulated to verify the proposed SOC and temperature balancing capabilities. The battery module contains 6 battery banks, and each battery bank has three lithium-ion battery cells as shown in figure 5.7. This battery bank can be connected to other banks in series to behave as an energy storage device with a significantly larger energy capacity. This section only focused on the SOC and thermal balancing between battery banks in one module. For module balancing, the management method is similar and the detail is shown in section 5.6. The entire battery system is given a constant input current that serves to either charge or TABLE 5.2 Initial SOCs and Temperatures during Discharging

\begin{tabular}{ccc}
\hline \hline Bank No. & SOC $(\%)$ & TEMPERATURE $\left({ }^{\circ} \mathrm{C}\right)$ \\
\hline 1 & 100 & 40 \\
2 & 95 & 35 \\
3 & 90 & 30 \\
4 & 85 & 20 \\
5 & 80 & 10 \\
6 & 75 & 0 \\
\hline \hline
\end{tabular}


Table 5.3 Initial SOCs and Temperatures during Charging

\begin{tabular}{ccc}
\hline \hline Bank No. & SOC $(\%)$ & TEMPERATURE $\left({ }^{\circ} \mathrm{C}\right)$ \\
\hline 1 & 10 & 40 \\
2 & 30 & 20 \\
3 & 20 & 30 \\
4 & 35 & 10 \\
5 & 25 & 0 \\
6 & 5 & 35 \\
\hline \hline
\end{tabular}

discharge the battery system over the course of 30,000 seconds, or 8.333 hours, at \pm 10 Ah. Ambient temperature is also introduced into the battery system so each bank also receives an ambient temperature value of $20^{\circ} \mathrm{C}$. Heat losses across the resistors of the electronic control module of each lithium-ion cell is also taken into account during the estimation process of temperature using the estimation tools in Simulink.

In this simulation, the balancing of temperature and SOC within a single bank is verified. Each battery bank within this module is given different initial temperatures and

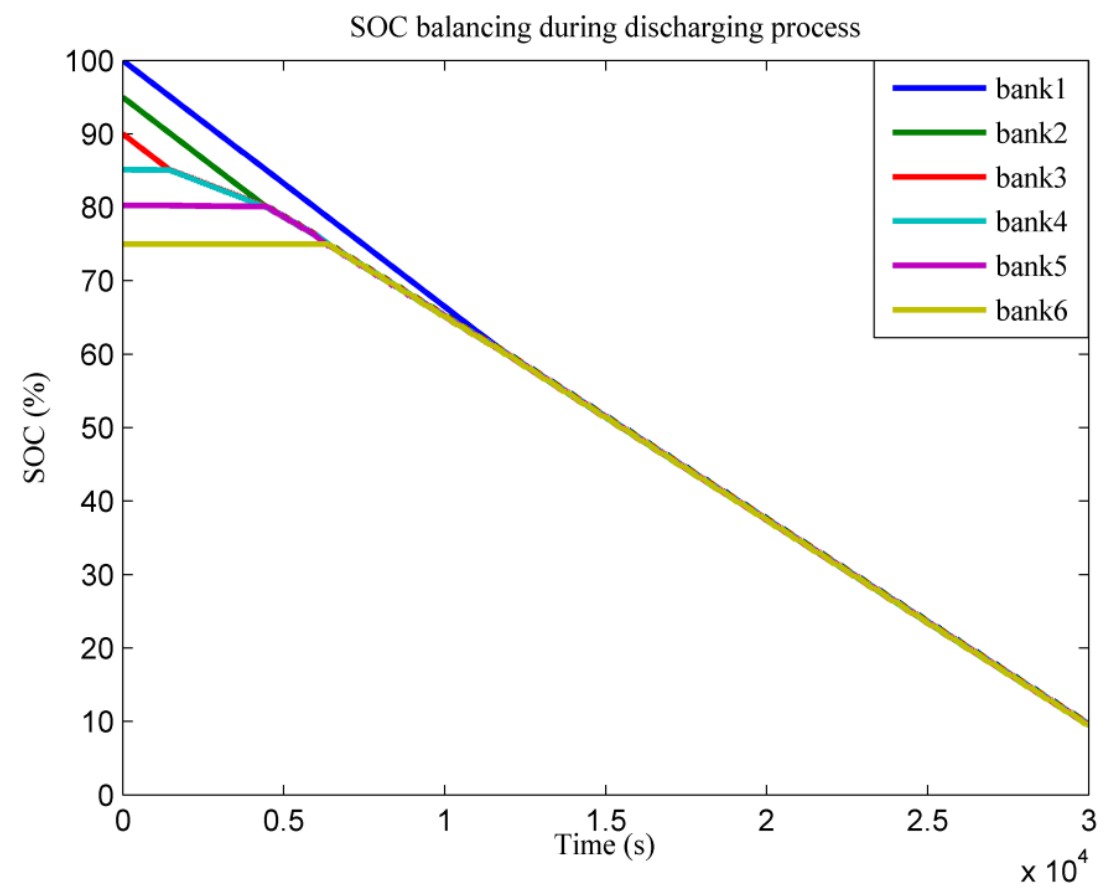

Figure 5.9 SOC Balancing during Discharging Cycle 
SOCs. The initial temperatures and SOCs of each bank during a discharge cycle of $10 \mathrm{Ah}$ and during a charging cycle of $10 \mathrm{Ah}$ are shown in Table 5.2 and Table 5.3, respectively.

Figure 5.9 shows successful balancing of the SOCs within the battery banks during the discharging process of $10 \mathrm{Ah}$ over the course of 30,000 seconds. The battery module's SOCs are fully balanced approximately 3 hours after initial discharging of the bank. Figure 5.10 shows that the various temperatures of each bank within the module also balance during this discharging cycle approximately 7 hours after initial discharging.

Similar results are observed during the charging cycle of 10 Ah over 30,000 seconds for the same battery module. Figure 5.11 shows that the SOC of the battery banks within this module balance themselves approximately 4 hours after initial charging.

Figure 5.12 displays the temperature balancing within the module during this cycle which



Figure 5.10 Temperature Balancing during Discharging Cycle 
shows the same results as the temperature balancing during the discharge process since the initial temperature values across the banks are kept the same.

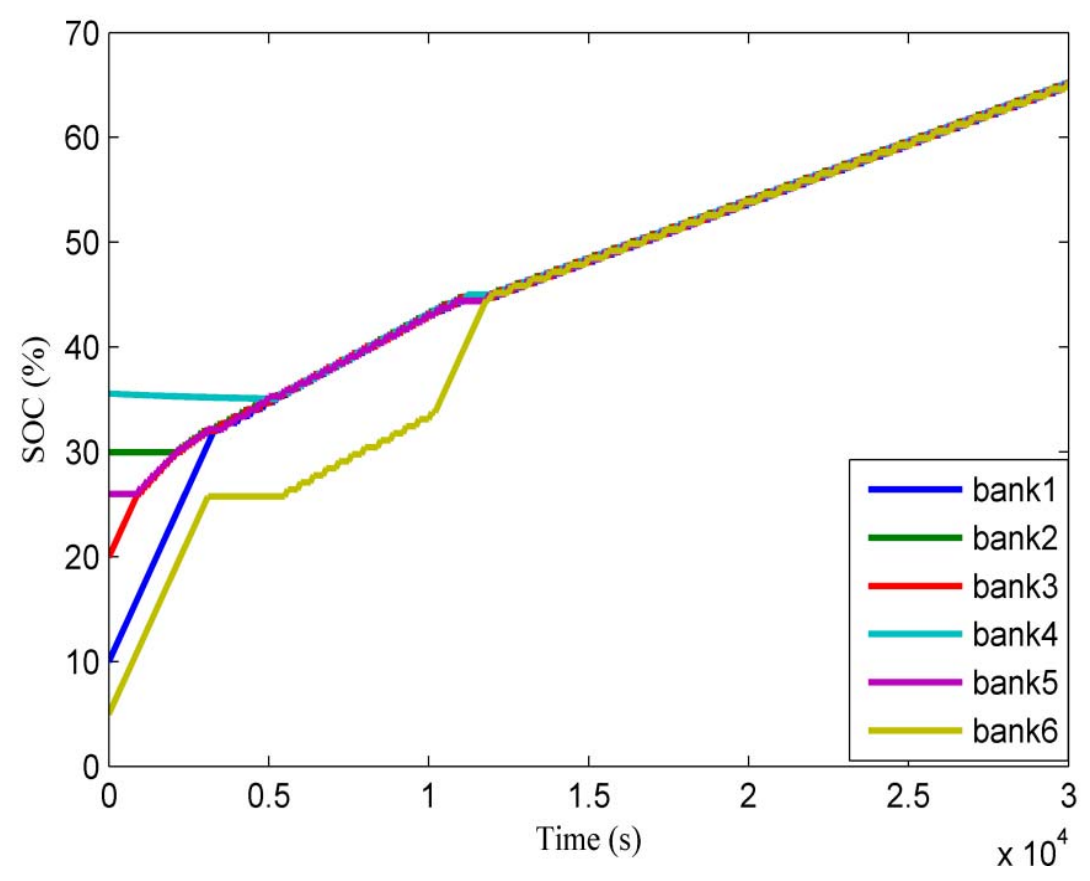

Figure 5.11 SOC Balancing during Charging Cycle

This section presented a fuzzy logic based battery module management system with self healing capabilities to protect itself from variations in the SOC and temperature of its battery banks. The power consumed by the resistive elements in the equivalent circuit model used for each lithium-ion cell, along with the ambient temperature introduced into the system, allows for accurate estimation of the temperature. The battery management system's effectiveness was demonstrated through simulations in Matlab for a battery management system that consisted of five battery modules, each with a $6 \times 3$ lithium-ion cell configuration and a switching topology that connects 6 banks in series with three parallel cells in each module. The simulation results demonstrated that the 
system was able to effectively regulate both SOC and temperature during charging and discharging cycles for long periods of time and increase the safety of the whole system.

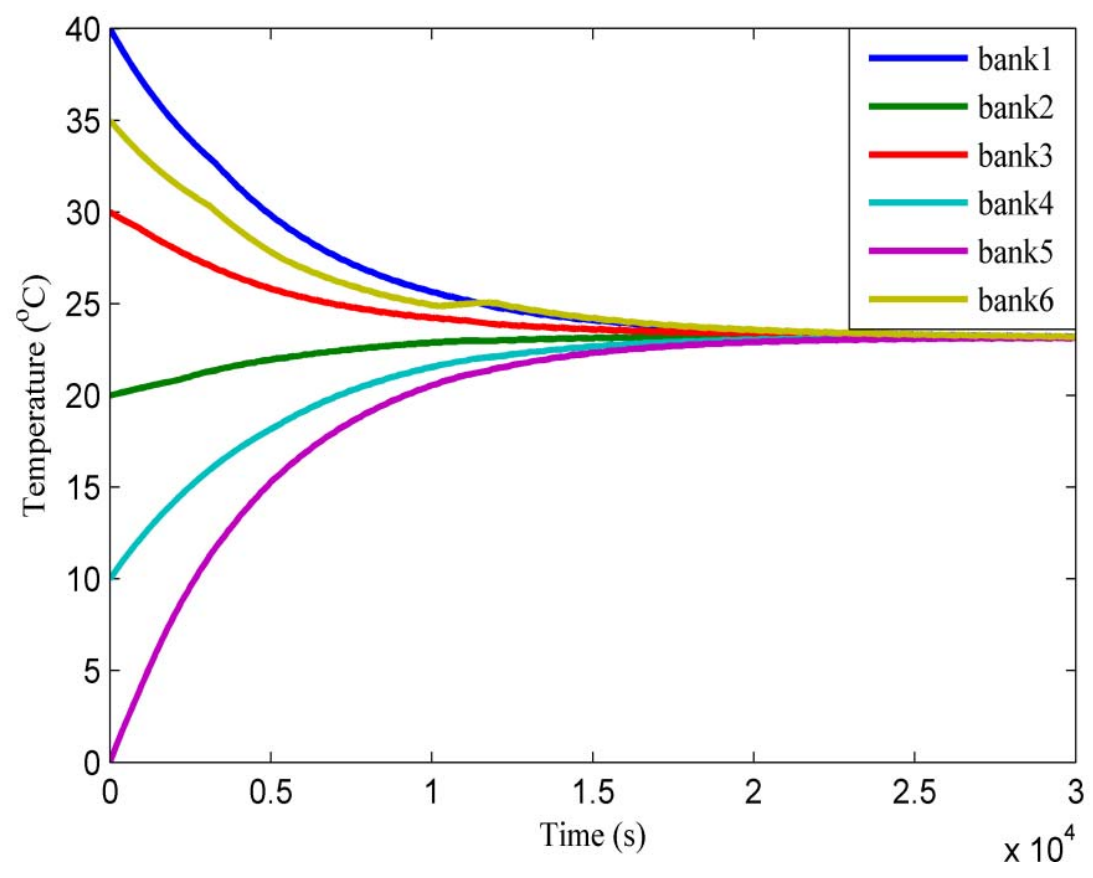

Figure 5.12 SOC Balancing during Charging Cycle

\subsection{Real-time Battery module Operation in Hybrid Power Systems with Droop Control}

This section presents the real-time coordinated control of distributed lithium-ion battery modules in hybrid power systems. It is common that in a hybrid power system, there are several energy storage systems connected to the DC side. To have several energy storage units work together and balance the SOC of each energy storage unit, an adaptive droop control based on the SOC is proposed. A central aggregator is used in this system to measure the DC bus voltage and collect the information about the SOC of each battery module. During the discharging process, battery modules with higher SOC will 
deliver more power compared with the ones with lower SOC. During the charging process, battery modules with lower SOC will absorb more power compared to those with a higher SOC. In this way, the SOC difference between different battery modules will be suppressed and the load can be equally shared by those distributed battery modules. A hardware platform is designed to verify the proposed control method.

\subsubsection{Droop control in hybrid power systems}

Modern hybrid power systems tend to be fairly distributed with multiple power generators on both the AC and DC side. Therefore, power systems engineers have to face a new scenario in which small distributed power generators and dispersed energy-storage devices have to be integrated together into the grid.

Controlling distributed energy resources units in the AC side based on droop characteristics is ubiquitous in the literature [90], [121]-[124]. Droop methods originate from the principle of power balance of synchronous generators in large interconnected power systems. An imbalance between the input mechanical power of the generator and its output electric active power causes a change in the rotor speed which is translated into a deviation in frequency. Similarly, output reactive power variation results in deviations in voltage magnitude. The frequency-power droop control method is inherent to the steady-state operation of conventional distributed generation units such as synchronous generators, and it can be artificially crafted for electronically interfaced distributed generation units.

In droop control, the relationship between real power/frequency and reactive power/voltage can be expressed as:

$$
\omega_{0}=\omega^{*}-K_{P}\left(P_{0}-P^{*}\right)
$$




$$
V_{0}=V^{*}-K_{Q}\left(Q_{0}-Q^{*}\right)
$$

Where the values $\omega^{*}$ and $V^{*}$ correspond to the reference values for angular frequency and voltage, respectively, $\omega_{0}$ and $V_{0}$ correspond to the measured output frequency and voltage of the distributed generation system, respectively. A similar convention is used for the active power and reactive power. The coefficients denote droop coefficients, and are determined based on steady-state performance criteria.

The example of conventional $P$ - $\omega$ and $Q-V$ droop characteristics are shown in figure 5.13. (a) and (b). Different distributed generation units may have different droop coefficients. Therefore, under certain situations, they can share the power appropriately according to their power generation capacity.

Droop control can also be performed on the DC side of the hybrid power system. The configuration of the DC side of the hybrid power system is shown in figure 5.14. The DC sources and distributed energy storage systems are connected to the DC bus through their DC-DC power electronics converters. In the steady state, the dc network will force the DC source terminal voltages to be close in value, but usually not equal because of

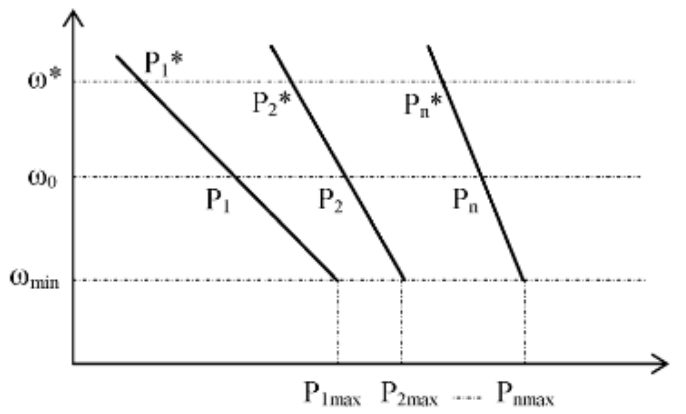

(a)

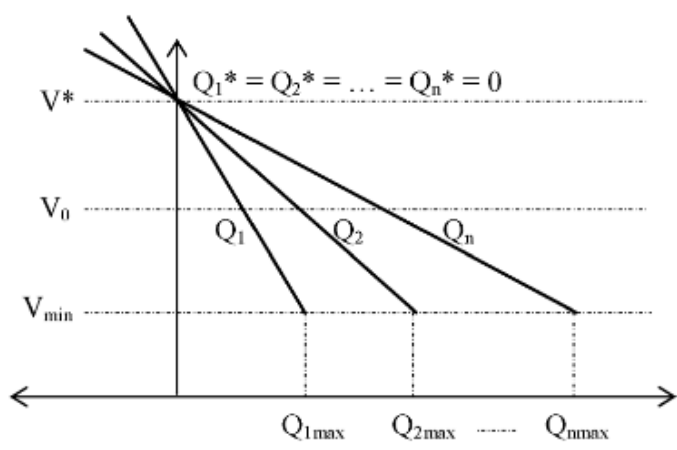

(b)

Figure 5.13 (a) $P-\omega$ droop characteristic. (b) $Q-V$ droop characteristic 
different virtual impedances of the DC-DC converters. By properly controlling the virtual impedance, the mismatch will cause active power sharing errors, which in the end force different distributed DC energy sources and energy storage systems to share the power similar to the reactive power sharing experienced by the AC subsystem [125]-[129].

\subsubsection{Adaptive droop control for multi battery module operation in hybrid power systems}

If several energy storage systems are connected to the DC bus of the hybrid power system individually through their own bidirectional DC-DC converter, a conventional PID controller couldn't be used to regulate the DC bus voltage. If all of them are used to regulate the DC bus voltage, they may conflict with each other and cause instability issues; if only one of them is used to regulate the DC bus voltage, distributing power flow becomes unclear to the other controllers, which may cause SOC unbalance between energy storage systems.



Figure 5.14 Hybrid power system DC subsystem configuration. 
In this section, adaptive droop control is used to regulate and dispatch power flow for multiple lithium-ion battery modules on the DC side of the hybrid power system. As shown in figure 5.14, five lithium-ion battery modules with $21 \mathrm{Ah}$ capacity and $51.8 \mathrm{~V}$ terminal voltage are connected to the same DC bus individually through their own bidirectional DC-DC converter. A PV emulator is connected to the DC bus as a DC side energy resource. Also, the $\mathrm{DC}$ bus is connected to the $\mathrm{AC}$ side through a bidirectional AC-DC inverter. The AC side of the hybrid power system can be connected either to the utility grid to operate in grid connected mode or to a local AC generator to operate in islanding mode. In steady state, the DC voltage can be influenced by several factors: abrupt change of PV output power because of sudden changes of solar irradiance, pulse load connection and disconnection, and power transformations between $\mathrm{AC}$ and $\mathrm{DC}$ sides. The detailed study of the power flow and cooperation of the power electronics devices in the hybrid power system is described in Chapter 8 . In this section, we only focus on the control of power flow in five battery modules.

During the operation, the DC bus voltage is monitored all the time. Whenever the DC bus voltage is above the rating value which is set as $100 \mathrm{~V}$, extra power needs to be transferred into the battery modules. On the other hand, battery modules need to inject

Table 5.4 Battery Modules Droop Coefficients

\begin{tabular}{ccc}
\hline \hline Bank Ranking No. & ChARging Droop COEFFICIENT & DisChARGING DroOP COEFFICIENT \\
\hline 1 & 2.0 & 4.0 \\
2 & 2.5 & 3.5 \\
3 & 3.0 & 3.0 \\
4 & 3.5 & 2.5 \\
5 & 4.0 & 2.0 \\
\hline
\end{tabular}


power to the DC bus once the DC bus voltage is lower than its rating value. In this way, the DC side of the hybrid power system will keep being operated with the rating voltage.

To intelligently distribute the power flow between the five lithium-ion battery modules with the function of self-balancing, a method of droop control with adaptive coefficients is proposed. The SOCs of the five battery modules are estimated by measuring the terminal voltages. With the SOC information, the five battery modules are ranked based on their SOC. With the SOC rank and the measured DC bus voltage, a central aggregator will calculate and assign the droop coefficient to each battery module. The droop coefficients are shown in Table 5.4, and based on the droop coefficients, the voltage-current droop characteristics of each battery module is shown in figure 5.15.

After assigning the droop coefficients, the reference current values are calculated. Those reference current values will be sent to the bidirectional DC-DC converters of those five lithium-ion battery modules. After receiving the reference current signal, the driver circuit will regulate the current flow through it. The detail of how to control the bidirectional DC-DC converter will be given in Chapter 8 Section 8.3.

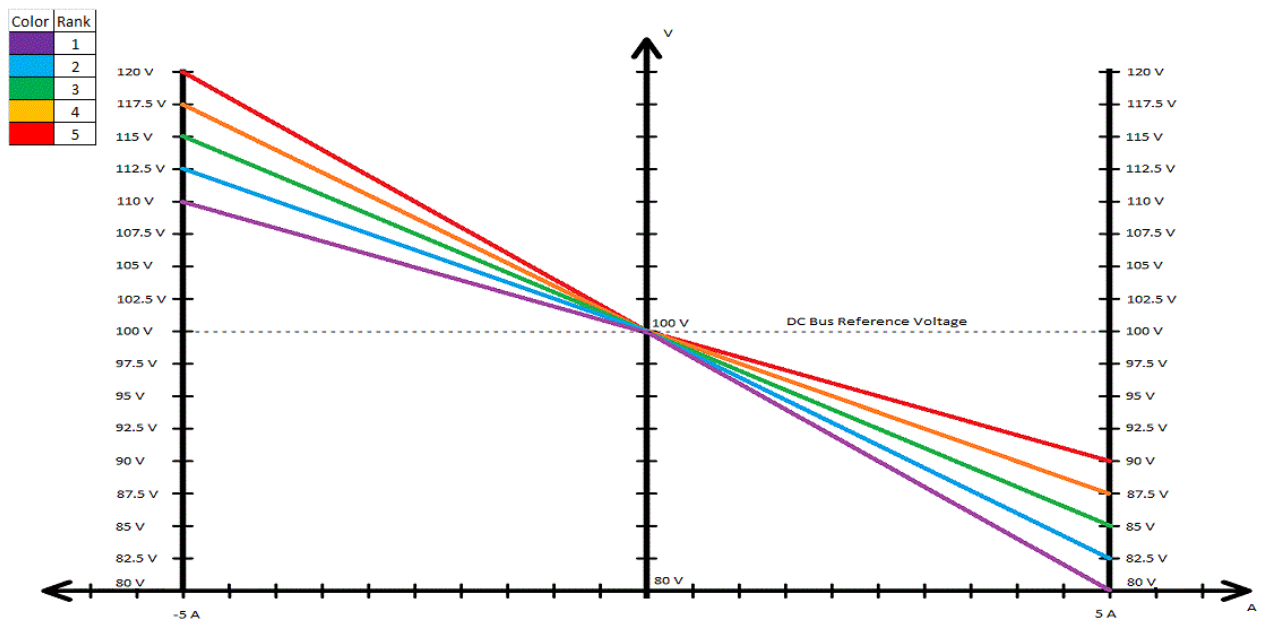

Figure 5.15 Voltage-current droop characteristics of each battery module 


\subsection{Conclusion}

This chapter presented a fuzzy logic based battery bank management system with self-healing capabilities to protect itself from variations in the state of charge and temperature of its battery banks. The power consumed by the resistive elements in the equivalent circuit model used for each lithium-ion cell, along with the ambient temperature introduced into the system, allows for accurate estimation of the temperature. The battery management system's effectiveness was demonstrated through simulations in Matlab for a battery module consists of $6 \times 3$ lithium-ion cell configuration and a switching topology that connects 6 banks in series with three parallel cells in each bank. The simulation results demonstrated that the system is able to effectively regulate both SOC and temperature during charging and discharging cycles for long periods of time and increase the safety of the whole system. Also, an adaptive droop control for distributed battery modules energy management with SOC balancing function is proposed. 


\section{Plug-in Hybrid Electric Vehicles Car Park Modeling and Design of Artificial Intelligent Power Management Algorithm}

This chapter proposes an intelligent workplace parking garage power management algorithm for plug-in hybrid electric vehicles (PHEVs). The system involves a developed smart PHEVs power charging controller, a photovoltaic (PV) panel farm, a DC distribution bus. Also, this parking garage is connected to the AC utility grid. Stochastic models of the power demanded by PHEVs in the parking garage and output power of the PV are presented. In order to limit the impact of PHEV's charging on the AC utility grid, a fuzzy logic power flow controller was designed. Based on their power requirements, PHEVs were classified into five charging priorities with different charging rates according to the developed fuzzy logic controller. The PHEVs charging rates can be calculated based on the predicted PV output power, the power demand by the PHEVs, the price of energy from the utility grid and some other factors. The system structure and the developed PHEVs smart charging algorithm are described with detail. Moreover, a comparison between the impacts of the charging process of the PHEVs on the grid with and without the developed smart charging technique is presented and analyzed. The developed system can dramatically limit the impacts of PHEVs on the utility grid, reduce the charging cost and at the same time maximize the utilization of the energy generated from the renewable energy farm. 


\subsection{Introduction}

PHEVs are gaining popularity due to several reasons; they are convenient, visually appealing, quiet, and produce less pollution in the environment. PHEVs have the potential to reduce fossil energy consumption, green-house gas emissions and increase the penetration of sustainable energy sources such as solar energy and wind energy into our daily lives [130]-[132]. Furthermore, most personal vehicles in the United States are parked more than $95 \%$ of the day and generally follow the same daily schedule [133]. Therefore, PHEVs can be used as mobile energy storage devices in the future. More than $75 \%$ of drivers in the United States travel less than 45 miles in their daily commute and since many of today's PHEVs can go up to 100 miles on a single charge, their implementation can be widespread. Battery technology continues to advance with batteries becoming smaller in size while storing more energy. It is forecasted that in North America PHEVs will be on the roads in large numbers in the very near future [134].

The increasing number of PHEVs can have a huge impact on the electric utility if properly designed smart charging techniques are not utilized. Uncoordinated and random charging activities could greatly stress the distribution system, causing several kinds of technical and economic issues such as suboptimal generation dispatches, huge voltage fluctuations, degraded system efficiency and economy, as well as increasing the likelihood of blackouts because of network overloads. In order to maximize the usage of renewable energy sources and limit the impacts of PHEVs' charging to the AC utility grid, a smart power flow charging algorithm and controller should be designed. Moreover, accurate PV output power and PHEV's power requirement forecasting models 
should be built. PHEVs need to participate in vehicle-to-grid (V2G) and vehicle-tovehicle (V2V) power transactions during the charging process. Fully controlled bidirectional AC-DC and DC-DC converters are needed in this system.

In [135] and [136], load management solutions for coordinating the charging process of multiple PHEVs in a smart grid system based on real-time minimization of total cost of generating the energy plus the associated grid energy losses were proposed and developed. However, they did not consider the inclusion of a renewable energy source in the system, which holds the implementation of these algorithms back since the concept of PHEVs involves obtaining the power to charge them from renewable energy sources. In addition, the control strategy did consider charging priority level, but the level is based on how much the owner of the PHEV is willing to pay, not the state of charge (SOC) of the PHEV's batteries, therefore the efficiency of V2V and V2G service is low.

In [137] and [138], an intelligent method for scheduling the use of available energy storage capacity from PHEVs is proposed. The batteries in these PHEVs can either provide power to the grid or take power from the grid to charge the batteries on the vehicles. However, the detail about the energy dispatch during charging and V2G process is not given. Also, the SOCs of the PHEV's batteries are not considered during the process.

A fully controlled bidirectional AC-DC converter has been designed and implemented in [139]. This converter has the capability of controlling the power flow between the $\mathrm{AC}$ and $\mathrm{DC}$ sides of the systems in both directions while operating at unity power factor and within acceptable limits of time harmonic distortion (THD) for the current drawn from the grid. Hence, the amount of power flowing in either direction can 
be set to a certain pre-set value while a controlled rectifier, working as a voltage rectifier, maintains the power balance as it is free to supply any power needed in the DC grid. In addition, a controlled DC-DC boost converter and a bidirectional DC-DC converter are proposed and tested in [140]-[142].

The objective of this chapter is to design a grid-connected hybrid DC PHEV charging parking garage system with fuzzy logic power flow control and PV panels. The goal is to limit the impact of PHEV's charging to the AC utility grid and maximize the utilization of power generated from PV panels. In this chapter, fuzzy logic based PHEV real-time energy management algorithms are proposed for smart charging of PHEVs in the car park with different scales. The real-time energy management algorithms may have different functions, such as maximizing the utilization of energy from renewable energy sources to charge PHEVs, reducing the PHEVs charging cost based on the utility grid energy price, or minimizing the charging impact to the utility grid with the consideration of local load.

This chapter is organized as follows; Section 6.2 presents the PHEVs car park energy consumption analysis including the details of PHEVs model classification, energy consumption per miles model, total daily energy consumption model, and PHEVs car park power requirement forecasting model. The details of the developed real-time fuzzy logic power flow controller are given in section 6.3. Section 6.4 presents two cases of study for a small scale PHEV car park charging process simulation with the purposes of maximizing the utilization of PV output power and reducing the charging cost. Section 6.5 gives a case of study for a medium scale PHEV car parking charging process 
simulation with the consideration of limiting the charging impact to the utility grid. Finally, the conclusion is given in section 6.6.

\subsection{PHEV Car Park System Description and Problem Formulation}

Consider a workplace parking garage DC hybrid power system equipped with a PV farm. Each workday various vehicles will park in the garage during their owner's working hours.

The schematic diagram of the system under study is shown in figure 6.1. As can be seen, the PHEVs, with their bidirectional DC-DC chargers, and the PV source, with its DC-DC regulating interface, share a common DC bus. Hence, the charging parking garage acts as a DC micro-grid that has the ability to send or receive power from the $\mathrm{AC}$ utility grid. The amount of power transferred between the AC and DC sides is determined according to the decisions from the developed energy management algorithm. Also, the active and reactive power flow is controlled separately by using the active and reactive

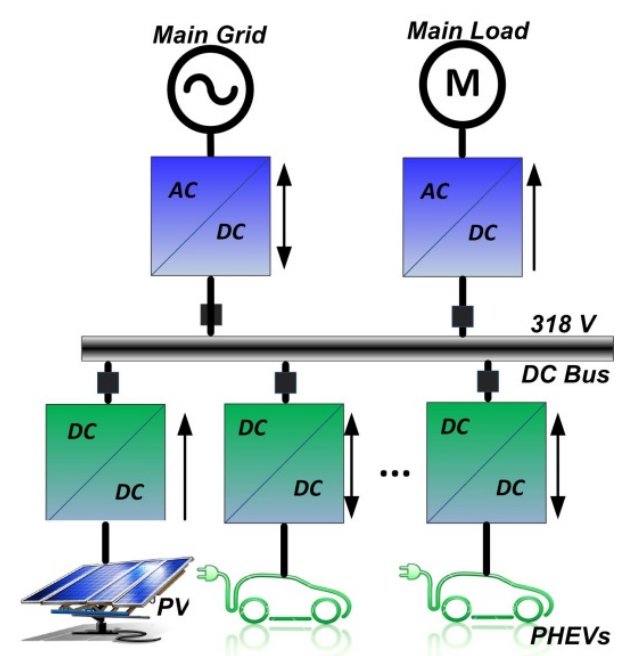

Figure 6.1 PHEVs carpark hybrid powers system schematic diagram 
power decoupling technique. More simulation and experimental results on this converter as well as the controlled rectifier were illustrated in [139].

In order to limit the impact of PHEVs charging to the AC utility grid while letting the PHEVs participate in the $\mathrm{V} 2 \mathrm{~V}$ and $\mathrm{V} 2 \mathrm{G}$ power transactions, the parking garage should have a smart charging algorithm that can adjust the charging rates for the PHEVs based on the utility AC energy prices $\left(E_{\text {price }}\right)$, power flow estimations $\left(P_{\text {grid }}\right)$, and PV farm output power $\left(P_{P V}\right)$. Since the hourly energy price is assumed to be known beforehand, it is essential to estimate $P$ grid, which is given by equation (6.1).

$$
P_{\text {grid }}=P_{P V}-P_{\text {total }}-\hat{P}_{\text {upcoming }}
$$

where

- $\quad P_{P V}$ is the estimated PV output power for the next period $T$;

- $P_{\text {total }}$ is the power needed by the PHEVs that are already parked in the parking garage;

- $\hat{P}_{\text {upcoming }}$ is the estimated power requirements for the upcoming PHEVs which will connect to the parking garage in the next period $T$.

In order to design the smart charging control algorithm, an accurate power requirement forecasting model is needed to estimate $P_{\text {grid. }}$. For the power flow control for the next period $T$, the charging rates for different PHEVs should be adjusted based on $E_{\text {price }}$ and $P_{\text {grid. }}$. Because the system is highly nonlinear, a fuzzy logic controller is a good choice for solving this issue.

Often times, the PHEVs in the parking garage will have different SOCs and different departure times, so their average constant power requirements will differ. Some 
PHEVs may need a huge amount of energy within a short period of time. These kinds of PHEVs should be classified into the high priority level. Other PHEV's SOCs are already high with departure times several hours later. These kinds of PHEVs should be classified into the lower priority level. Therefore, a priority classification should be designed for the PHEVs.

In order to develop an accurate PHEV parking system model, it is essential to estimate the probability density function (PDF: a function that describes the relative likelihood for this random variable to take on a given value [143]) of the power needed by each PHEV when it is connected to the parking lot, $\hat{P}_{\text {PHEV }}$. This variable is based on the PHEV models (battery capacities, energy consumptions), parking duration times in the parking garage, and the daily travel distances. Therefore, to model a PHEVs parking garage system, the PDF of those parameters should be found first.

To avoid serious damage, the batteries of the PHEVs should not be over discharged. PHEVs have the capability of using both electric energy and fossil fuel energy. The PHEV stops using electric energy when the SOC of its battery is below $10 \%$. Therefore, the electric energy of a PHEV that can be used before its next charge is $70 \%$ of the total battery capacity. If the energy consumption is more than this value, the PHEV will use gas. If the total energy consumption for a certain PHEV before the next charge is less than $70 \%$ of its battery capacity, the energy needed by it for the next charge is $M \times E_{m}$. Otherwise, the energy needed by it is $70 \%$ of its battery capacity. The constant charging power needed by this PHEV is given below in (2) and (3). In order to find $\hat{P}_{\text {PHEV }}$, the distribution of daily travel distance and daily parking duration time need to be obtained first. 
If total energy consumption is less than $70 \%$ of the battery capacity:

$$
\hat{P}_{\text {PHEV }}=\frac{M_{d} \times E_{m}}{D_{t}-A_{t}}
$$

If total energy consumption is equal or more than $70 \%$ of the battery capacity:

$$
\hat{P}_{P H E V}=\frac{70 \% \times B_{c}}{D_{t}-A_{t}}
$$

where

- $M_{d}$ is the driver's daily travel distance;

- $A_{t}$ is the PHEV's arrival time;

- $D_{t}$ is the PHEV's departure time;

- $E_{m}$ is the PHEV's energy consumption per mile;

- $B_{c}$ is the PHEV's battery capacity.

\subsubsection{PHEV model classification}

The PHEVs differ in size, battery capacity, and energy consumption per mile. The specific details are shown in Table 6.1. Whenever a PHEV is connected to the parking garage, the owner of it will set the next departure time and the system will make a record of this. To not influence the driving behaviors of the PHEV owners, at the departure time, the SOC of the batteries are expected to be at least $80 \%$ of its full capacity. In order to take battery protection into consideration, the SOC of the PHEV's battery shouldn't go below a certain limit. Therefore, when a certain PHEV's SOC is below a certain limit, it should not be involved in the discharging process needed in the V2G and V2V services. But during charging process, it is operated as normal. By doing this, the battery banks in the PHEVs can be effectively protected from over discharging, which will extend the lifetime the battery banks. 


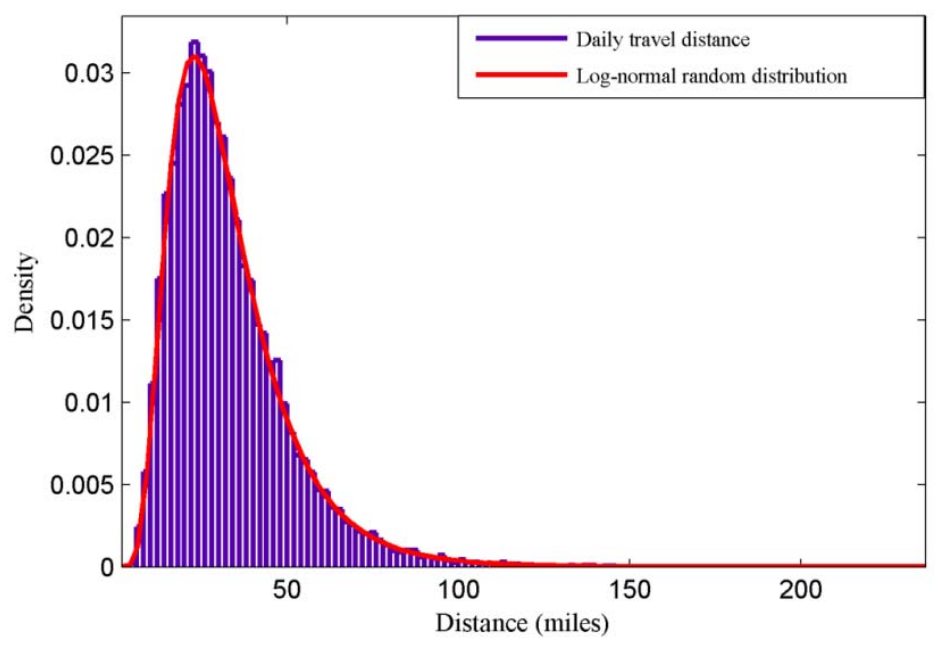

Figure 6.2 The PDF of the daily travel distance

\subsubsection{PHEV daily travel distance modeling}

Based on known driving pattern statistics, the average yearly total miles driven in the United States is 12,000 miles with $50 \%$ of drivers driving 25 miles per day or less, and $80 \%$ of drivers driving 40 miles or less. A log normal distribution with $\mu_{m}=3.37$, $\sigma_{m}=0.5$ is selected to approximate the PDF of $M_{d}$, which shows that the total yearly driving distance average is 12,018 miles, $48 \%$ of the vehicles drive 25 miles or less each day, and $83 \%$ of the vehicles drive 45 miles or less each day, which closely approximates the driving performance results from [130]. The distribution function for $M_{d}$ is given in equation (6.4). The PDFs of the $M_{d}$ is shown in figure 6.2.

Table 6.1 Parameters For Phevs In Different Size

\begin{tabular}{cccc}
\hline \hline PHEVs model & Percentage & Battery capacity $(\mathrm{kWh})$ & $\begin{array}{c}\text { Energy consumption } \\
\text { per mile }(\mathrm{kWh} / \mathrm{mile})\end{array}$ \\
\hline $\begin{array}{c}\text { compact sedan } \\
\text { full-size sedan }\end{array}$ & $32.5 \%$ & $10-20$ & 0.2 \\
$\begin{array}{c}\text { mid-size SUV } \\
\text { or pickup } \\
\text { full-size SUV } \\
\text { or pickup }\end{array}$ & $37.5 \%$ & $20-30$ & 0.3 \\
\hline \hline
\end{tabular}




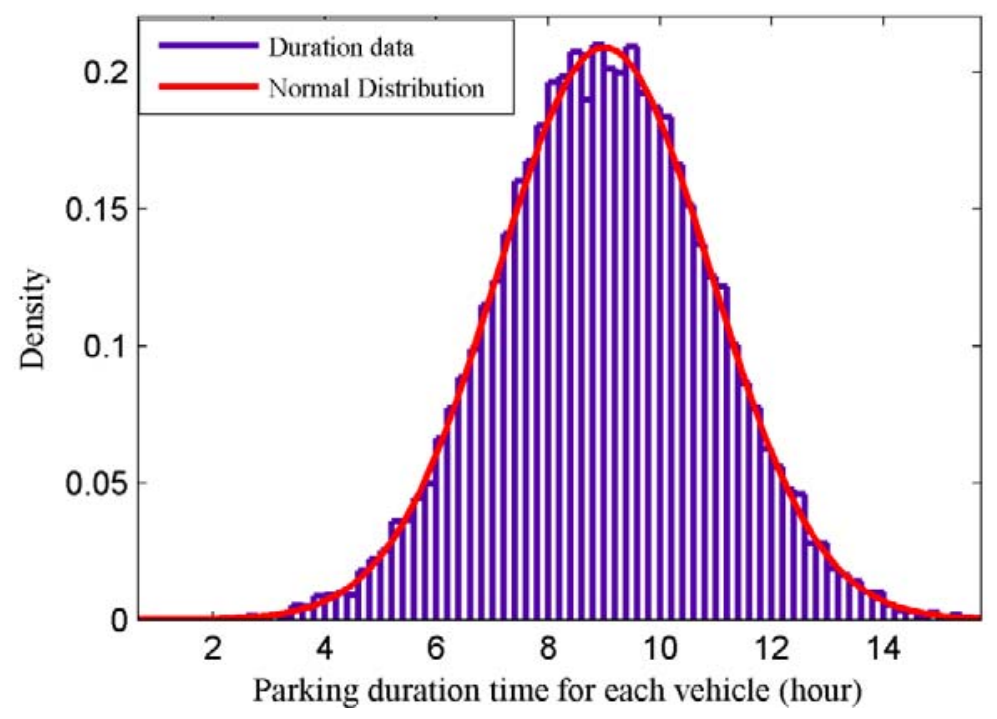

Figure 6.3 The PDF of the daily parking duration

$$
f_{X}\left(x ; \mu_{m}, \sigma_{m}\right)=\frac{1}{x \sigma_{m} \sqrt{2 \pi}} \exp \left\{-\frac{\left(\ln x-\mu_{m}\right)^{2}}{2 \sigma_{m}{ }^{2}}\right\}
$$

\subsubsection{PHEVs parking duration modeling}

In this work, the parking garage is located by the workplace of a company whose office hours are from 9:00 am to 6:00 pm. Based on the Central Limit Theorem (the conditions under which the mean of a sufficiently large number of independent random variables, each with finite mean and variance, will be approximately normally distributed [144]), the distribution of the PHEVs arrival and departure time is shown in Table 6.2. With the PDFs of $A_{t}$ and $D_{t}$, the joint probability density function of $D_{t}-A_{t}$ can be found,

Table 6.2 Arrival And Departure Times Distribution Parameters

\begin{tabular}{ccccc}
\hline \hline & \multicolumn{2}{c}{ Arrival } & \multicolumn{2}{c}{ Departure } \\
\hline Parameter & Weekday & Weekend & Weekday & Weekend \\
$\mu_{T}[h]$ & 9 & 11 & 18 & 15 \\
$\left(\sigma_{T}\right)^{2}[h]$ & 1.2 & 1.5 & 1.2 & 1.5 \\
\hline \hline
\end{tabular}




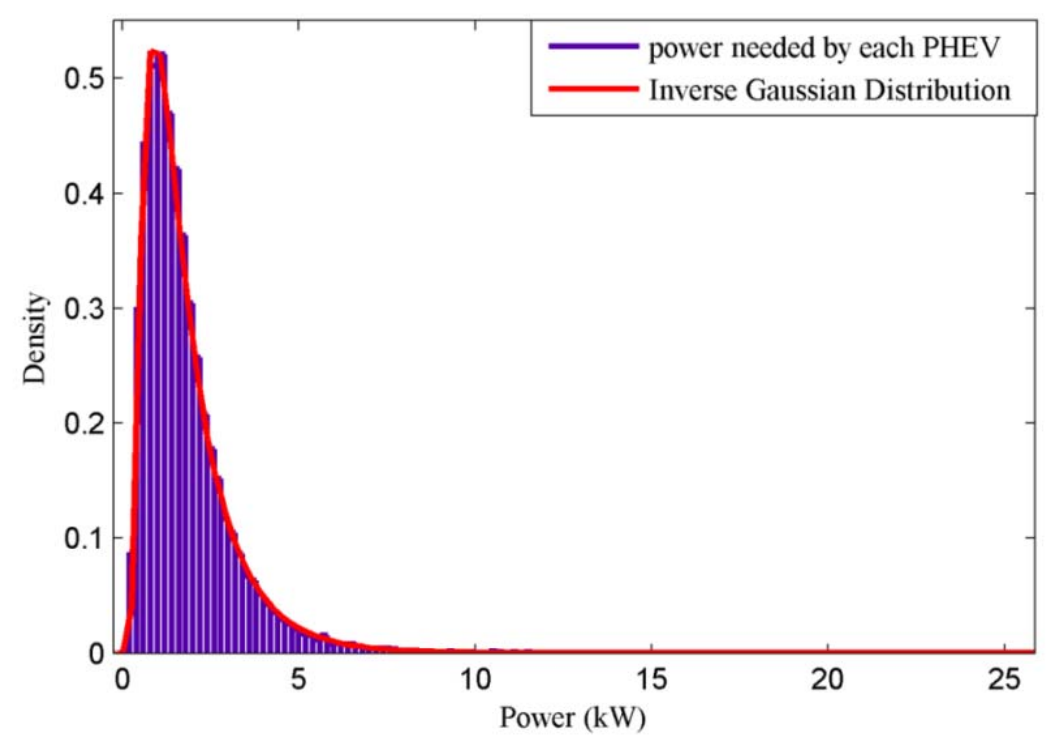

Figure 6.4 Power needed by each PHEV when connected to the parking garage which is the daily parking duration time. It is a normally distributed random variable with $\mu_{d}$ and $\sigma_{d}=1.92 a$. The PDF of the daily parking duration is shown in figure 6.3.

\subsubsection{PHEV charging power modeling}

With the PDF of daily duration time, PDF of daily travel distance, and power consumptions of each class of PHEVs, we can use MATLAB's statistic distribution fitting toolbox and Monte Carlo simulation with 30000 samples to find the PDF of constant power needed by each PHEV when it is connected to the parking lot, $\hat{P}_{P H E V}$, as an inverse Gaussian distribution with $\mu_{p}=1.573$ and $\lambda_{p}=3.652$. The distribution function for $\hat{P}_{\text {PHEV }}$ is given in equation (6.5). The PDF of the $\hat{P}_{\text {PHEV }}$ is shown in figure 6.4.

$$
f_{X}\left(x, \mu_{p}, \lambda_{p}\right)=\sqrt{\frac{\lambda_{p}}{2 \pi x^{3}}} \exp \left\{-\frac{\lambda_{p}}{2 \mu_{p}{ }^{2} x}\left(x-\mu_{p}\right)^{2}\right\}
$$




\subsubsection{PHEV car park power requirement forecasting model}

After getting the probability distribution function of $\hat{P}_{\text {PHEV }}$, the forecasting model of power needed by PHEVs in the parking system is built. Together with the forecasting model of the power generated by renewable energy sources and hourly price of the energy from the utility grid, a real-time smart parking system is established. For instance, at a certain time $t$, the SOC of the PHEVs already parked in the parking lot and their power requirements are already known. In order to forecast the power needed by the PHEVs that will arrive during the upcoming period $T$, the following equation (6.6) can be used.

$$
\hat{P}_{\text {upcoming }}=\int_{t}^{t+T} f_{A_{t}}\left(x, \mu_{A_{t}}, \sigma_{A_{t}}\right) d t \times N P \times \hat{p}_{\text {PHEV }} \text { avg }
$$

where

- $N P$ is the total number of PHEVs that will park in the parking lot this day;

- $f_{A_{t}}\left(x, \mu_{A_{t}}, \sigma_{A_{t}}\right)$ is the PDF of the arriving time $A_{t}$;

- $\hat{P}_{P H E V \_a v g}$ is the average constant power requirement for all PHEVs when they are connected to the parking lot. $\hat{P}_{P H E V_{-} \text {avg }}$ can be calculated from the PDF of $\hat{P}_{P H E V}$.

\subsection{Fuzzy Logic Based Real Time Energy Management Algorithm}

In the previous section, the details of how to build the model of the parking

garage and find the PDF of the $\hat{P}_{P H E V}$ are given. Together with the stochastic model of PV and hourly energy price of the AC utility grid, a smart charging algorithm with a fuzzy logic power flow controller is designed. The flowchart is shown in figure 6.5. 


\subsubsection{PHEV Charging Priority Levels classification}

Different PHEVs with different SOCs and power requirements should be charged at different rates. For example, a PHEV is connected to the parking lot at 9:00 am with a departure time of 6:00 pm and a SOC of 65\%. The average constant power required by this PHEV is small. At the same time, another PHEV is connected to the parking lot also at 9:00 am, but will leave at 10:30 am and the SOC is only 10\%. This PHEV's average constant power requirement is larger than the previous one, which means its charging condition is also more urgent. In order to reduce the impact of the PHEVs' charging to the AC utility grid, at a certain time, different PHEVs should be charged at different

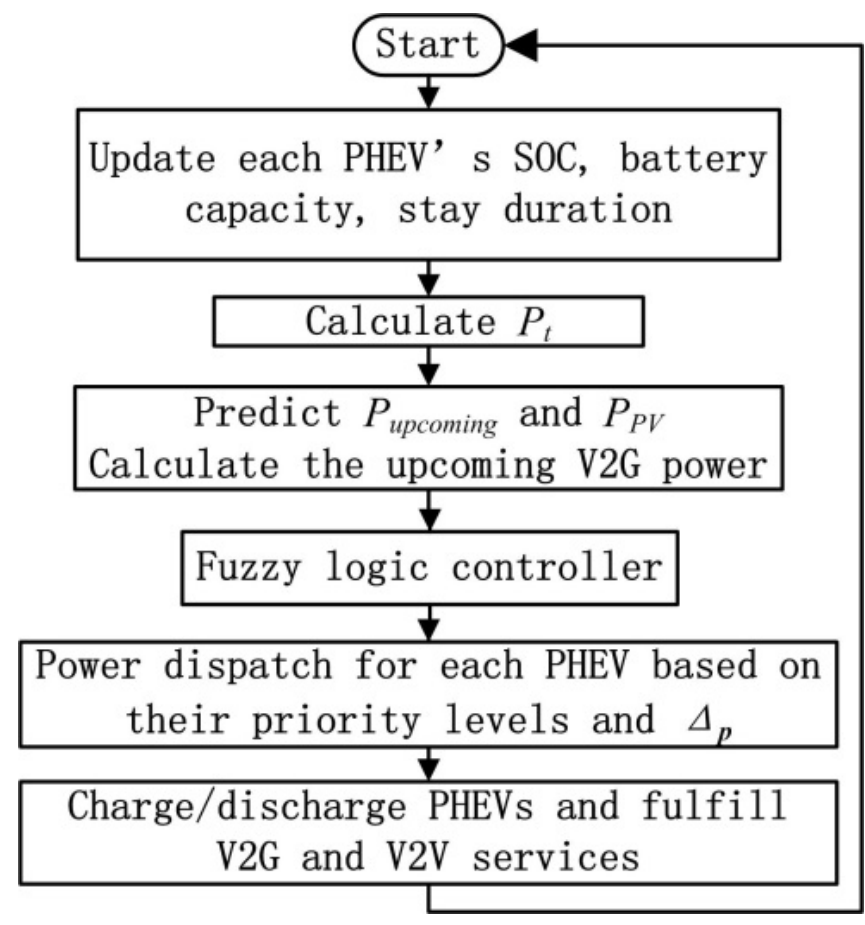

Figure 6.5 The flow chart of the developed real time fuzzy logical charging controller. 
rates. Furthermore, since the first PHEV will stay in the parking lot for more than 8 hours, it can be viewed as an energy storage device during this period. For instance, if at a certain time the energy price is below the daily average price and the PV generates more power than the total PHEV's requirements. The extra power can be saved in this first PHEV as backup energy. By doing so, the priority level of this PHEV decreases. At another time during this period, the price of the utility grid energy could be high and the power generated by the PV can't meet the total load plus the total PHEV's power requirements, so instead of buying power with a high price from the utility grid, the parking system can get the backup energy from the first PHEV. By doing so, the priority of this PHEV will increase. During the entire day, all the PHEV's priorities are varying with their SOCs, thus energy can be delivered between V2G and V2V. The five charging priorities are shown in Table 6.3.

The PHEV's charging priority levels are only dependent on their power requirements. Also, because of the bidirectional power flow converter, PHEVs can be charged and discharged, so their charging priority levels are varying with time. PHEVs in levels 1, 2, and 3 can only be charged. Those PHEVs either need a lot of energy (such as having a SOC of only $10 \%$ when connected to the parking station) or will leave in a short time but still have not met the owner's charging requirement (such as having a SOC of only $65 \%$ and departing in half an hour). PHEVs in level 4 and 5 can be discharged to fulfill the V2G and V2V services. These PHEVs will continue staying in the parking lot for longer durations. The various SOCs of the PHEVs will change over time, thus PHEVs in lower priority levels can jump to the higher levels of priority and vice versa. 
With the charging index, $\delta_{p}$, the charging rates of PHEVs in levels 1-5 are given in (6.7)-(6.11).

$$
\begin{aligned}
& p_{c h \text { arg } \text { ing_rate }}=12, \\
& p_{\text {ch arg } \text { ing_rate }_{\text {rate }}}=9+3 \times \delta_{p}, \\
& p_{\text {ch } \text { arg } \text { ing_rate }}=4+4 \times \delta_{p}, \\
& p_{\text {ch arg } \text { ing_rate }_{\text {rate }}}=0+5 \times \delta_{p}, \\
& p_{\text {ch } \text { arg } \text { ing_rate }}=-3+5 \times \delta_{p} .
\end{aligned}
$$

\subsubsection{Fuzzy logic controller design}

The charging rates of PHEVs at different priority levels for the next period varies based on the forecasting of the power generated by the PV panel, the forecasting of the power needed by the upcoming PHEVs, the price of the utility energy grid, and the power needed by the current PHEVs. Without the V2V and V2G services, the power flow in the next period between the AC utility grid and the hybrid parking system can be calculated by using equation (6.1).

There are several variables can be used to decide the charging rates of the PHEVs in different priority levels. For a different purpose, different variables can be chosen. For example, if the designed car park is aimed to reduce greenhouse gas emissions by maximizing the utilization of renewable energy resources to charge the PHEVs, then the forecasted renewable energy farm output power can be used to decide the charging rates.

Table 6.3 Charging Rates for Different Charging Levels

\begin{tabular}{cccc}
\hline \hline Priority level & Power requirement & Maximum charging rate & Minimum charging rate \\
\hline Level 1 & $p \geq 15 k W$ & $12 \mathrm{~kW}$ & $12 \mathrm{~kW}$ \\
Level 2 & $10 k W \leq p<15 \mathrm{~kW}$ & $12 \mathrm{~kW}$ & $6 \mathrm{~kW}$ \\
Level 3 & $5 k W \leq p<10 \mathrm{~kW}$ & $8 \mathrm{~kW}$ & $0 \mathrm{~kW}$ \\
Level 4 & $2 k W \leq p<5 \mathrm{~kW}$ & $5 \mathrm{~kW}$ & $-5 \mathrm{~kW}$ \\
Level 5 & $p<2 k W$ & $2 \mathrm{~kW}$ & $-8 \mathrm{~kW}$ \\
\hline \hline
\end{tabular}


For another example, if the designed car park is focused on reducing the charging cost of the PHEVs, then the utility grid energy price can be used as a factor to influence the charging rates of the PHEVs. Further, to fulfill multiple functions, two or more factors can be used together as the inputs to decide the charging rates.

However, the charging rates of PHEVs have no direct relationship with factors such as renewable energy farm output power, the PHEV car park's hybrid system energy gap, and the utility grid energy price. Therefore, a conventional linear controller like the PID controller can't solve the problem. Therefore, a fuzzy logic controller is utilized in this study to smartly control the PHEVs' charging process.

In this chapter, two fuzzy logic controllers are proposed. The first controller is used to maximize the utilization of renewable energy resources, while at the same time limiting the PHEVs' charging impact to the utility grid. The second controller is aimed at minimizing the PHEVs' charging cost, while at the same time limiting the PHEVs' charging impact to the utility grid. Both controllers will generate a charging rate index $\delta_{P}$, which will be used to adjust the charging rates of PHEVs at different priority levels based on equation (6.12).

$$
P_{b a t t, i}= \begin{cases}12 & , \text { for level1 } \\ 9+3 \times \delta_{P} & , \text { for level 2 } \\ 4+4 \times \delta_{P} & , \text { for level 3 } \\ 0+5 \times \delta_{P} & , \text { for level 4 } \\ -3+5 \times \delta_{P} & , \text { for level 5 }\end{cases}
$$

\subsubsection{Maximizing the utilization of renewable energy sources}

During the whole day, all the PHEVs' priorities are varying with their SOCs and departure times. The first input of the fuzzy logic controller is $P_{p v}$, the forecasted output 
energy of the PV farm for the next period, which is derived from the forecasting result of the Neural Network. The second input of fuzzy logic controller is $P_{g a p}$, the forecasted energy gap between the output energy from the PV farm and the PHEVs' energy requirements for the rest of the day, which can be calculated by using equation (6.13), where $t_{\text {current }}$ is the current time.

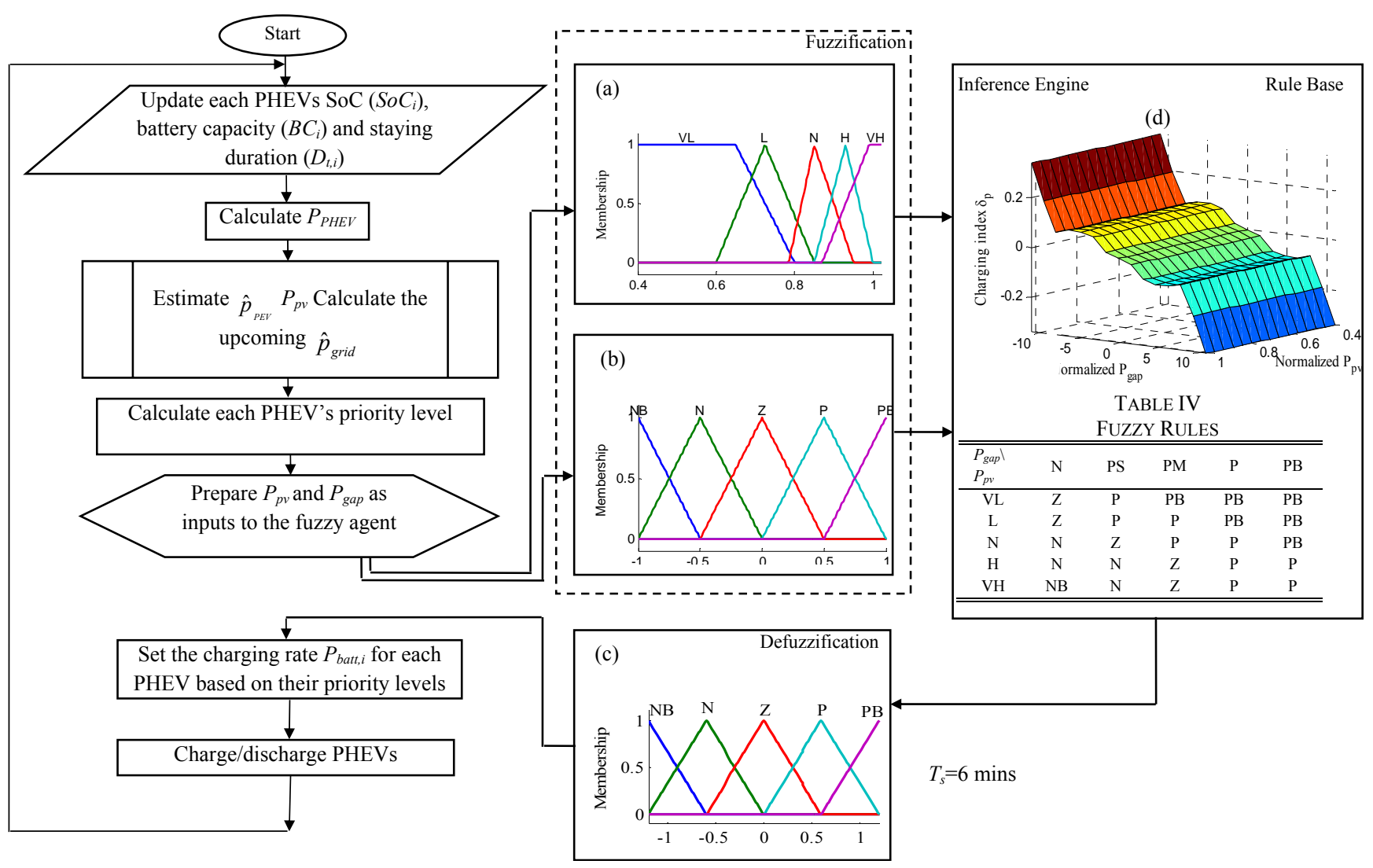

Figure 6.6 Flow chart of Fuzzy Logic based controller for maximizing the utilization of power from $\mathrm{PV}$.

$$
P_{\text {gap }}=A \times \beta \int_{t_{\text {current }}}^{t=24} u(t) \times t d t-\sum_{i=1}^{N}\left(\frac{0.8-S o C_{i}}{t_{\text {current }}-D w_{\text {ti }}}\right)
$$

The PV farm next period energy output, $P_{p v}$, is described as "very low", "low", "normal", "high" and "very high". Similarly, the energy gap, $P_{\text {gap }}$, is described as "negative large", "negative", "zero", "positive", and "positive large". The method 
implemented for defuzzification is centroid based. Within the model, the minimum and maximum are used for "AND" and "OR" operators, respectively. The charging rate index, $\delta_{P}$, is described as "negative big", "negative", "zero", "positive" and "positive big". For convenience, the inputs and outputs are normalized. The output $\delta_{P}$ is in the range of $[-1,1]$. After getting $\delta_{P}$, the charging of the PHEVs at different levels can be calculated. The flow chart of the smart charging algorithm is shown in figure 6.6.

\subsubsection{Minimizing the PHEVs' charging cost}

The price of energy for the next period, $E_{\text {price, }}$, and the next period forecasting power flow, $P_{\text {grid }}$, are used as the two inputs of the real time Mamdani-type fuzzy logic power flow controller to determine the charging index $\delta_{p}$, which will determine the charging rates of PHEVs at different priority levels. The power flow between the AC utility grid and the DC system, $P_{\text {grid, }}$ is described as "negative", "positive small", "positive medium", "positive" and "positive big". Similarly, the energy price, $E_{p r i c e}$, is described as "very cheap", "cheap", "normal", "expensive", and "very expensive". The method implemented for defuzzification is centroid based. Within the model, minimum and maximum are used for "AND" and "OR" operators, respectively.

Table 6.4 Fuzzy Logic Rules

\begin{tabular}{cccccc}
\hline \hline$p_{\text {grid }} E_{\text {prica }}$ & Negative & Positive Small & Positive Medium & Positive & Positive Big \\
\hline Very & $\mathrm{Z}$ & $\mathrm{P}$ & $\mathrm{PB}$ & $\mathrm{PB}$ & $\mathrm{PB}$ \\
Cheap & $\mathrm{Z}$ & $\mathrm{P}$ & $\mathrm{P}$ & $\mathrm{PB}$ & $\mathrm{PB}$ \\
Cheap & $\mathrm{N}$ & $\mathrm{Z}$ & $\mathrm{P}$ & $\mathrm{P}$ & $\mathrm{PB}$ \\
Normal & $\mathrm{N}$ & $\mathrm{N}$ & $\mathrm{Z}$ & $\mathrm{P}$ & $\mathrm{P}$ \\
Expensive & $\mathrm{N}$ & $\mathrm{Z}$ & $\mathrm{P}$ & $\mathrm{P}$ \\
Very & $\mathrm{NB}$ & $\mathrm{N}$ & & & \\
Expensive & & & &
\end{tabular}


The output of the fuzzy controller is the index $\delta_{p}$, which is used for adjusting the charging rates of PHEVs in different priority levels. The parameter $\delta P$ is described as

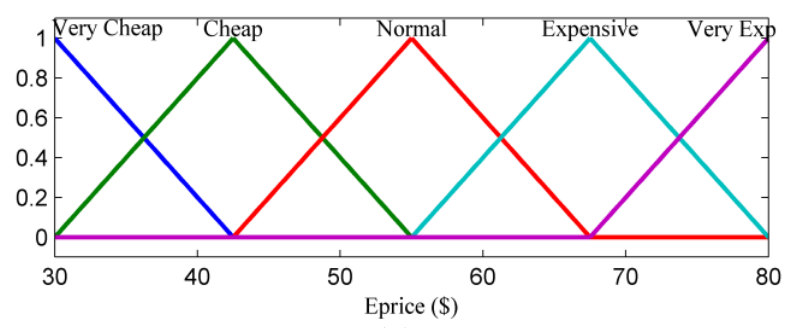

(a)

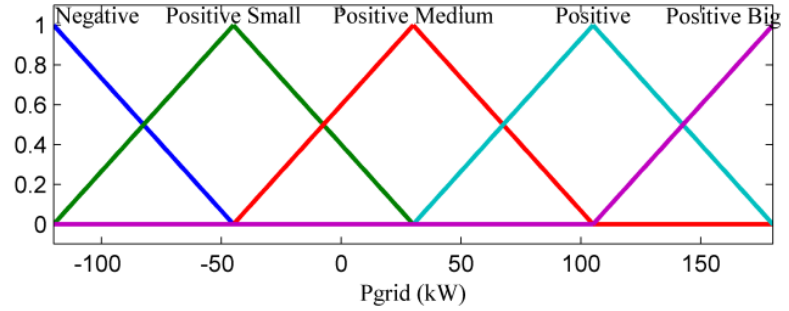

(b)

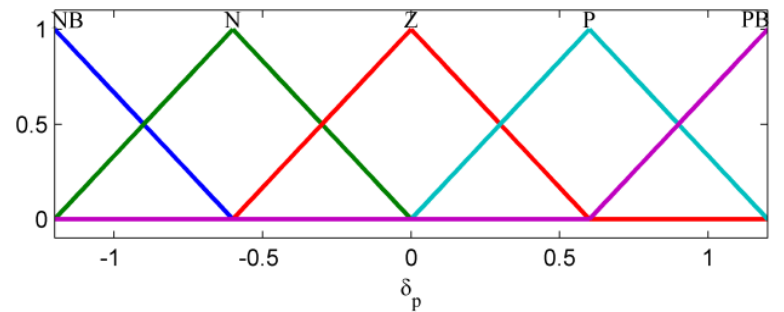

(c)



(d)

Figure 6.7 Membership functions and surface of the logic controller's rules base. (a) Power flow; (b) Energy price; (c) Power flow control index, (d) Surface of the fuzzy logic controller's rules base. 
"NB", "N", "Z", "P" and "PB", which stand for negative big, negative, zero, positive and positive big. The Mamdani-type model based fuzzy rules of the fuzzy logic power flow controller is given in Table 6.4.

The membership functions of $E_{\text {price, }}, P_{\text {grid }}, \delta_{p}$, and the surface of the fuzzy logic controller's rules are shown in figure 6.7 (a), (b), (c), and (d). Using the sign of $\delta_{p}$ along with the PHEVs' priority level, each PHEVs' charging rate will be calculated, which is sent to its corresponding bidirectional DC-DC converter. If $P_{\text {batt }, i}$ is positive for the $i^{\text {th }}$ PHEV, then the battery of this PHEV will be charged, whereas if $P_{b a t t, i}$ is negative for the $i^{\text {th }}$ PHEV, then its battery will be discharged.

\subsection{Fuzzy logic based PHEV smart charging case studies}

In this section, the fuzzy logic based PHEV smart charging algorithms are tested for two different cases. The first case applied the fuzzy logic controller presented in section 6.3.3 to a small scale PHEV car park to maximize the utilization of power generated from a PV farm. The second case applied the fuzzy logic controller presented in 6.3.4 to a small scale PHEV car park to minimize the PHEVs charging costs.

\subsubsection{Small scale PHEV car park smart charging with maximum utilization of renewable energy}

In this case study, a small scale model of a $300 \mathrm{~V}$ workplace parking garage hybrid power system equipped with a $3350 \mathrm{~m}^{2} \mathrm{PV}$ panel and 350 parking positions is used. Each work day around 300 PHEVs will park in the garage from 9AM to 6PM. The

fuzzy logic smart charging controller uses the PV farm output power and the hybrid 
power system power gap as the inputs to maximize the utilization of renewable energy resources while at the same time limiting the PHEVs' charging impact.

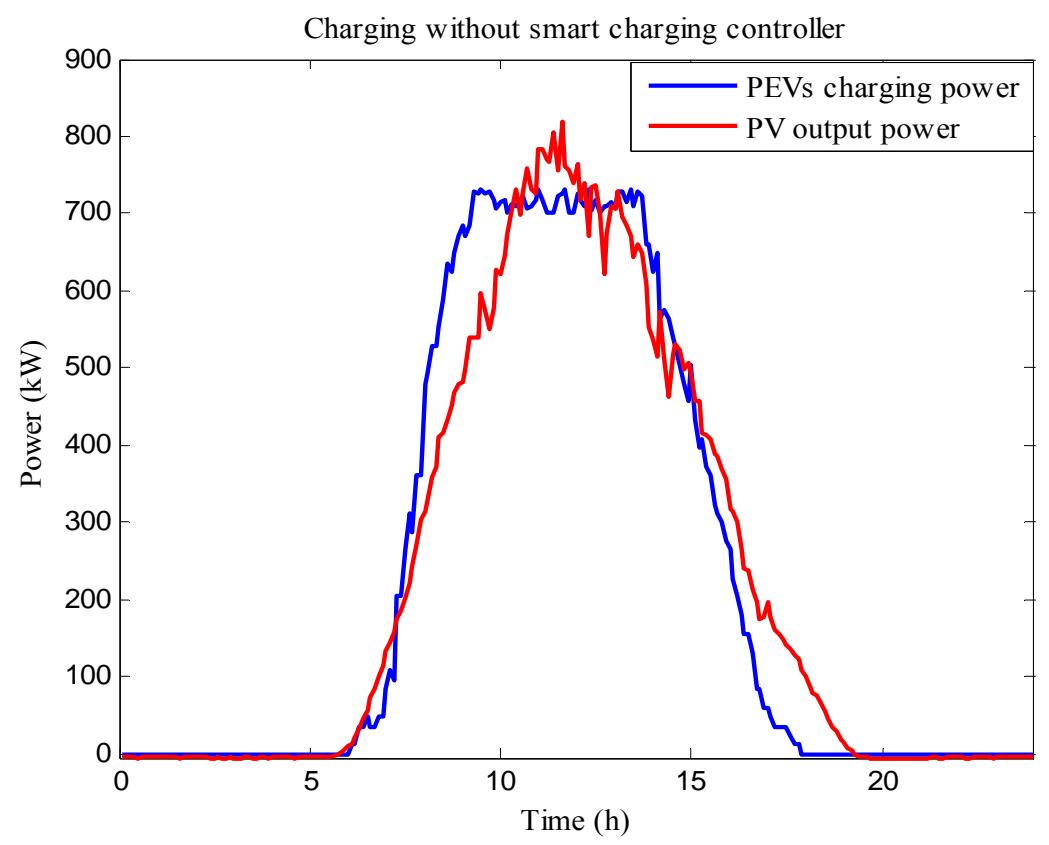

Figure 6.8 PHEVs charging power without real-time optimal charging control.

Two simulations of the proposed PHEV car park model were done. The first simulation is the PHEVs' charging power flow in the parking garage without real-time charging optimal control. The simulation result is shown in figure 6.8. In this situation, whenever a PHEV is connected to the parking garage, it will be charged with a $10 \mathrm{~kW}$ charging rate and not stop charging until the SOC of its' battery reaches $80 \%$. From the simulation it is clear that the peak happens around 9:00 AM because most of the PHEVs arrive around this time every day. The peak lasts until 3:00 PM, at which point the charging power decreases sharply since most of the PHEVs' SOCs have met their requirement. In the morning, the energy gap between the PHEVs' charging power and the PV output power need to be supported by the AC utility grid, which will in turn impact the AC utility grid. Also, the energy gap in the afternoon will require the car park to 
inject power to the grid, which may cause variation in the local AC utility grid frequency. Meanwhile, the power generated by the PV farm is not being fully utilized.

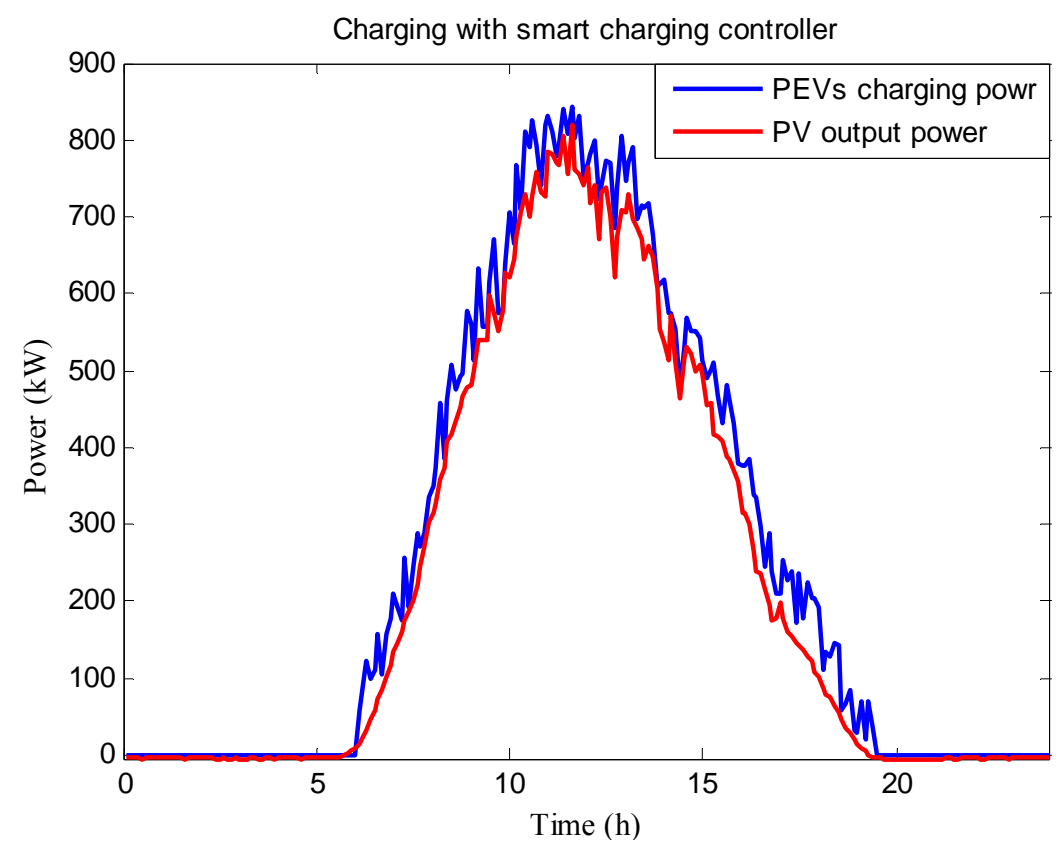

Figure 6.7 PHEVs charging power with real-time optimal charging control

The second simulation contains real-time fuzzy logic charging optimal control. The simulation result is shown in figure 6.9. In the second simulation, the fuzzy logic smart charging controller is applied to the system, and the PHEVs' charging can closely follow the changing output power from the PV farm. In this way, the car park power system doesn't need to absorb or inject too much power to the AC utility grid, which will limit the impact to the AC utility grid. This is because this hybrid PHEV charging system is able to support the PHEVs' power requirements for the entire day with its' PV farm, which allows it to avoid being a big load to the AC utility grid. Also, PHEV owners will pay less since most of the energy used to charge their PHEVs is generated by the renewable energy sources. At the same time, this smart charging controller will maximize 
the utilization of renewable energy from the PV farm, which will decrease the fossil energy consumption, and consequently decrease the greenhouse gas emissions.

Based on a comparison of the simulations in this case, with the PV farm short term output power forecasted by ANN, together with the PHEVs' power requirements estimated by the stochastic model, a fuzzy logic smart charging controller can be applied to maximize the utilization of the PV farm output energy to charge the PHEVs. From the simulation results, the proposed fuzzy logic control algorithm is capable of getting enough power from the PV farm to support the PHEVs in the car park while at the same time limiting the impact of the PHEVs' charging to the AC utility grid and minimizing greenhouse gas emissions.

\subsubsection{Small scale PHEV car park smart charging with consideration of local load and utility grid energy price}

In this case study, a small scale model of a $318 \mathrm{~V}$ DC workplace parking garage hybrid power system equipped with a $75 \mathrm{~kW}$ PV panel and 350 parking positions is used. Each work day around 300 vehicles will park in the garage from 9:00 am to 6:00 pm. Of the 300 vehicles, approximately $60 \%$ of them are PHEVs. The fuzzy logic smart charging controller uses the utility grid hourly energy price and the hybrid power system power gap as the inputs to minimize the PHEVs' charging cost while at the same time limiting the PHEVs' charging impact.

Two simulations of the proposed PHEV car park model were done. The first simulation represents the power flow between the utility grid and the DC hybrid PHEV parking garage without real-time charging optimal control and the second simulation contains real-time fuzzy logic charging optimal control. Both simulations are under the 
same conditions: same number and types of PHEVs, same departure and arrival times, same hourly energy price, and same power generated by the PV panels.



Figure 6.8 Hourly power flow from AC grid without optimal controller.

The simulation of the power flow during the daytime and the PHEVs' SOCs at departure time from the parking garage without an optimal charging method is shown in figure 6.10 and Figure 6.11. Whenever a PHEV is connected to the parking garage, it will be charged with a constant rate of $10 \mathrm{~kW}$. It will not stop charging until the SOC of its battery reaches $80 \%$. From the simulation it is clear that the peak happens around 9:00 am because most of the PHEVs arrive around this time every day. The peak is near 700 $\mathrm{kW}$ and the power flow above $300 \mathrm{~kW}$ lasts from 7:30 am to 11:20 am, more than three and a half hours. After 1:30 pm, the charging stops because all the PHEVs that are parked in the garage at that time already meet the charging requirement. After 1:30 pm there is no power flow between the AC utility grid and the parking garage because there aren't any new PHEVs connected to the parking garage. But at that time, the PV's output power is still high while the energy price is cheap. It's not a good time to sell power to the AC 
utility grid, but the parking garage without optimal charging control doesn't have any other option than selling the power. From Figure 6.11 it is clear that all the PHEVs' SOCs are above $80 \%$ at their departure times since all of them are charged with the same charging rates.

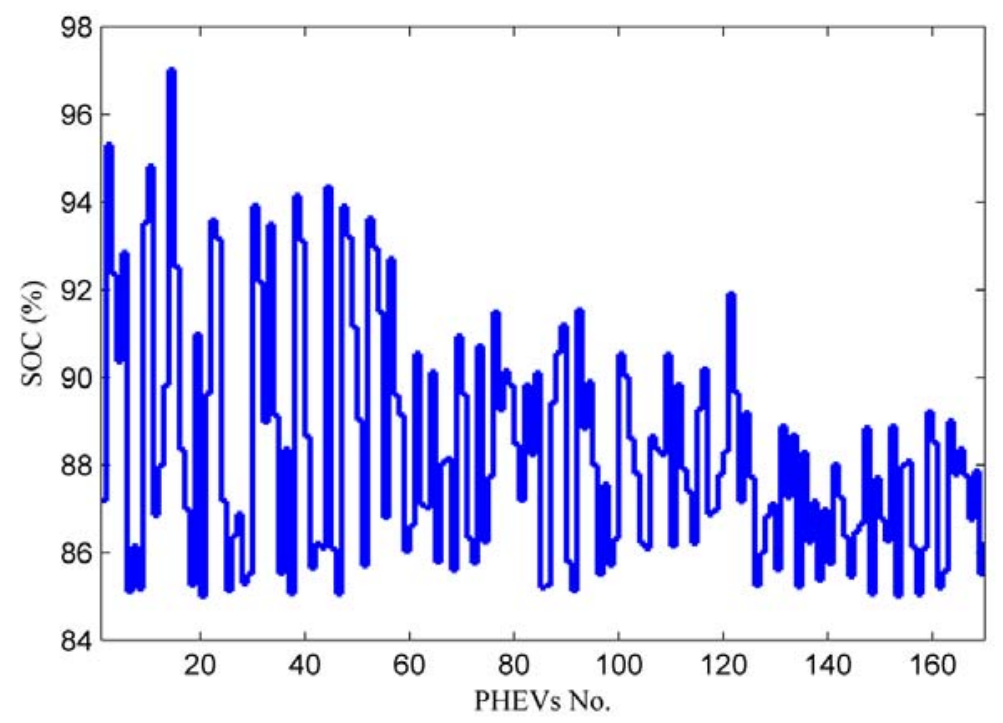

Figure 6.9 PHEVs' SOCs at their departure time without optimal controller.

The simulation of the power flow during the daytime and the departing PHEVs' SOC for the parking garage with an optimal fuzzy logic charging controller is shown in figure 6.12 and figure 6.13. From figure 6.12 it is clear that the peak of the power flow from the $\mathrm{AC}$ utility grid to the smart parking garage is limited to $300 \mathrm{~kW}$ and the power flow, which is above $250 \mathrm{~kW}$, only lasts from 9:30 am to 11:20 am and partly in the afternoon around 4:00 pm, all together no more than two and half hours.

Furthermore, when the energy price is high, the power flow from the AC side will decrease, which happens around 5:00 pm. Also, when the PV output power is above a certain amount, power flow from the $\mathrm{AC}$ utility grid to the smart charging garage will 
decrease because more PHEVs will be charged from the power generated by the PV. From figure 6.13 we can see all the PHEVs' SOCs are above $80 \%$ at their departure times, which also meets the charging requirements.

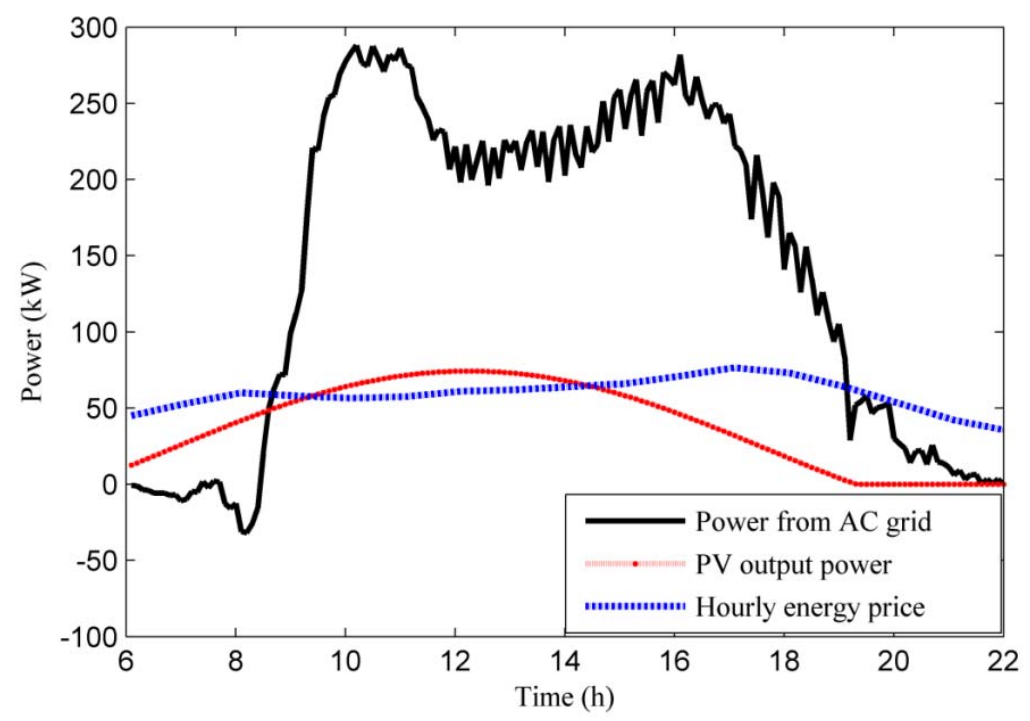

Figure 6.10 Hourly power flow from the AC grid with optimal controller.

Figure 6.14 shows the variation of a randomly chosen PHEVs' SOC during the charging process with the optimal fuzzy logic charging controller. This PHEV is connected to the parking garage at 8:18 AM, and the departure time is 5:12 PM. When this PHEV is connected to the parking garage, the SOC is around 28\%, and the PHEV's owner enters the departure time as 5:30 PM. So the charging system can calculate the real time average power required for this PHEV. The duration time is long at the beginning of the day from 8:00 am to 10:00 am so the PHEV's average power requirement is low with a classification of level 4 or 5 . At this time the price of energy is high, therefore instead of buying power from the $\mathrm{AC}$ utility grid, the parking garage uses the energy stored in this PHEV to charge other PHEVs in the higher levels of priority. This is why the SOC of the PHEV is decreasing during this period. From 10:00 am to 1:30 pm, the AC utility grid 
energy price is low so more power is bought from the $\mathrm{AC}$ side and since $\delta_{p}$ is positive, this PHEV's charging rate is positive. However, the duration time is still long so the priority level is low and the charging rate is low. The priority levels increase at around 2:00 pm, when its departure time is near. At this time the charging rate is higher than before. This charging rate is maintained until 5:12 pm, when the SOC is already above $80 \%$ and the departure time is very close. This PHEV no longer participates in V2G or $\mathrm{V} 2 \mathrm{~V}$ power transactions and the SOC remains constant from then on.

This case presented a model of a PHEV workplace car park charging infrastructure with a grid-connected hybrid DC power system involving renewable sources. To forecast the next period power flow, accurate PHEV and PV power stochastic models were developed. The fuzzy logic power flow controller was designed to control the real-time power flow. A new power dispatch method based on PHEV priority levels and a real-time PHEV charging algorithm was developed. The simulation results show that the optimal power flow control algorithm can maximize the utilization of PV output

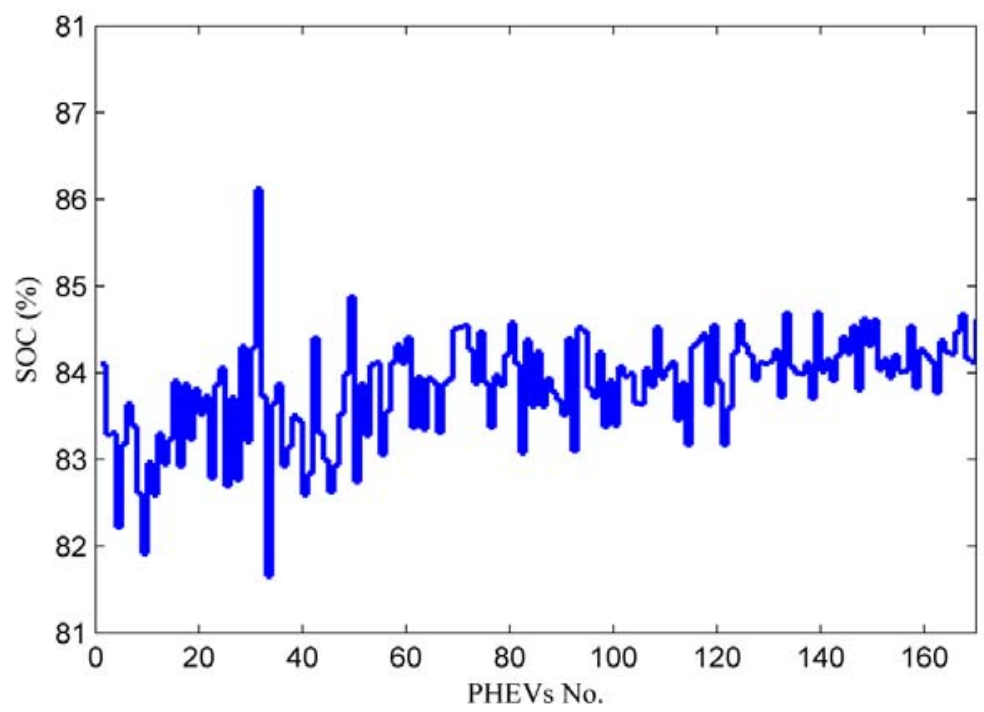

Figure 6.11 PHEVs' SOCs at their departure time with optimal controller. 
power for charging of PHEVs and simultaneously greatly decrease the impacts on the grid. At the same time, the PHEVs' SOCs at their departure times are all above the charging requirement. The system presented in this case benefits both the $\mathrm{AC}$ utility grid and PHEV owners.

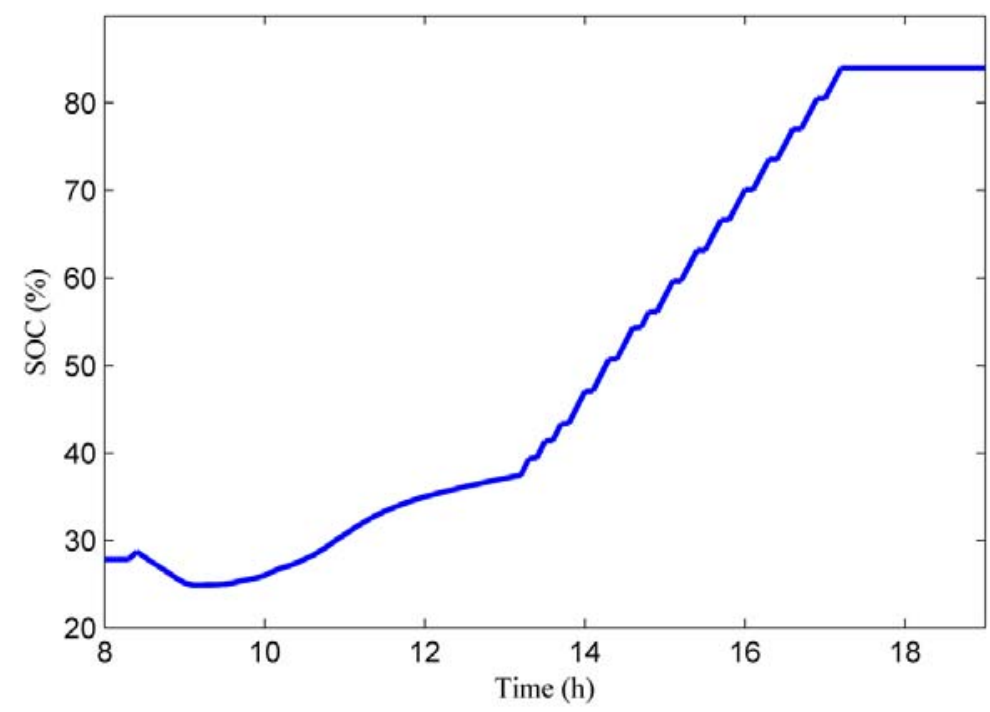

Figure 6.12 PHEV's SOC change during the smart charging process.

\subsection{Fuzzy logic smart charging controller for a medium scale PHEV car park}

In this section, the charging process for a medium scale PHEV car park in a workplace such as a university campus which contains 1500 parking spaces is studied. This car park is an IEEE 69-bus radial distribution system with feeder voltage of 12.66 $\mathrm{kV}$ and total load base case of 3.82 MW and 2.85 MVar. We assume that $60 \%$ of the 1500 vehicles parking daily in this car park are PHEVs. These 900 PHEVs are the ones managed in this study. The fuzzy logic smart charging controller uses the AC utility grid hourly energy price and the hybrid power system's power gap as the inputs to minimize the PHEVs' charging cost while at the same time limiting the PHEVs' charging impact. 
Both winter and summer local load patterns are used for this case study, and the charging impact to the distribution system with different PHEV connection points is studied.

\subsubsection{Local daily load consideration}

Based on different values of $\delta p$, the PHEVs in different priority levels in every sample will be charged with different charging rates. In order to further limit the impacts of the charging process of the PHEVs to the AC utility grid, the charging algorithm may also take into consideration the local load curve. For example, in the winter the daily load curve has two peaks; the first peak takes place around 9:00 AM and the second peak takes place around 9:00 PM. Moreover, the load is almost at its minimum around 3:00 PM, so that is the best time to charge the PHEVs if we want to decrease the impact of the PHEVs to the AC utility grid.

Another index $\sigma_{p}$ will be used to adjust the power flow between the AC utility grid and the hybrid parking system. This index is designed based on the load curve at the main feeder. When the load demand is relatively low, below $60 \mathrm{~kW}$ in our design, we don't need to consider the local load and the charging rate will just be dependent on $\delta p$, which is the output of the fuzzy controller depending on the PHEVs' demand and the energy tariff. If the load demand is between $60 \mathrm{~kW}$ and $80 \mathrm{~kW}, \sigma_{p}$ will be decreasing linearly from 1 to 0.9 . When the load demand exceeds $80 \mathrm{~kW}$, the local load is high and near the peak, so a quadratic equation with 0.9 at load $80 \mathrm{~kW}$ and 0 at load $100 \mathrm{~kW}$ will be used, which can limit the impact from the charging parks to the AC utility grid by decreasing the charging rate. This is mathematically represented by: 


$$
\sigma_{P}= \begin{cases}1 & , \overline{\hat{P}_{L}} \leq 0.6 \\ -0.5 \hat{P}_{L}+1.3 & , 0.6<\overline{\hat{P}_{L}} \leq 0.8 \\ -{\overline{\hat{P}_{L}}}^{2}-2.7 \hat{\hat{P}}_{L}+3.7 & , 0.8<\overline{\hat{P}_{L}} \leq 1\end{cases}
$$

where, $\bar{P}_{L}$ is the normalized load data. After obtaining $\sigma_{P}$, the final charging rates for PHEVs in different priority levels can be achieved by using the following set of equations:

$$
P_{\text {batt }, i}= \begin{cases}12 \times \sigma_{P} & , \text { for level1 } \\ \left(9+3 \times \delta_{P}\right) \times \sigma_{P} & , \text { for level2 } \\ \left(4+4 \times \delta_{P}\right) \times \sigma_{P} & , \text { for level3 } \\ \left(0+5 \times \delta_{P}\right) \times \sigma_{P} & , \text { for level4 if } \delta_{P} \geq 0 \\ \left(0+5 \times \delta_{P}\right) \times\left(2-\sigma_{P}\right) & , \text { for level4 if } \delta_{P}<0 \\ \left(-3+5 \times \delta_{P}\right) \times \sigma_{P} & , \text { for level5 if } \delta_{P} \geq 0 \\ \left(-3+5 \times \delta_{P}\right) \times\left(2-\sigma_{P}\right) & , \text { for level5 if } \delta_{P}<0\end{cases}
$$

\subsubsection{PHEV uncontrolled charging impact to the AC utility grid}

In order to examine the operation of the developed real-time energy management algorithm, the IEEE 69-Bus Radial Distribution System was used. Figure 6.15 shows a single line diagram of this system which includes 69 buses and 7 lateral branches. The

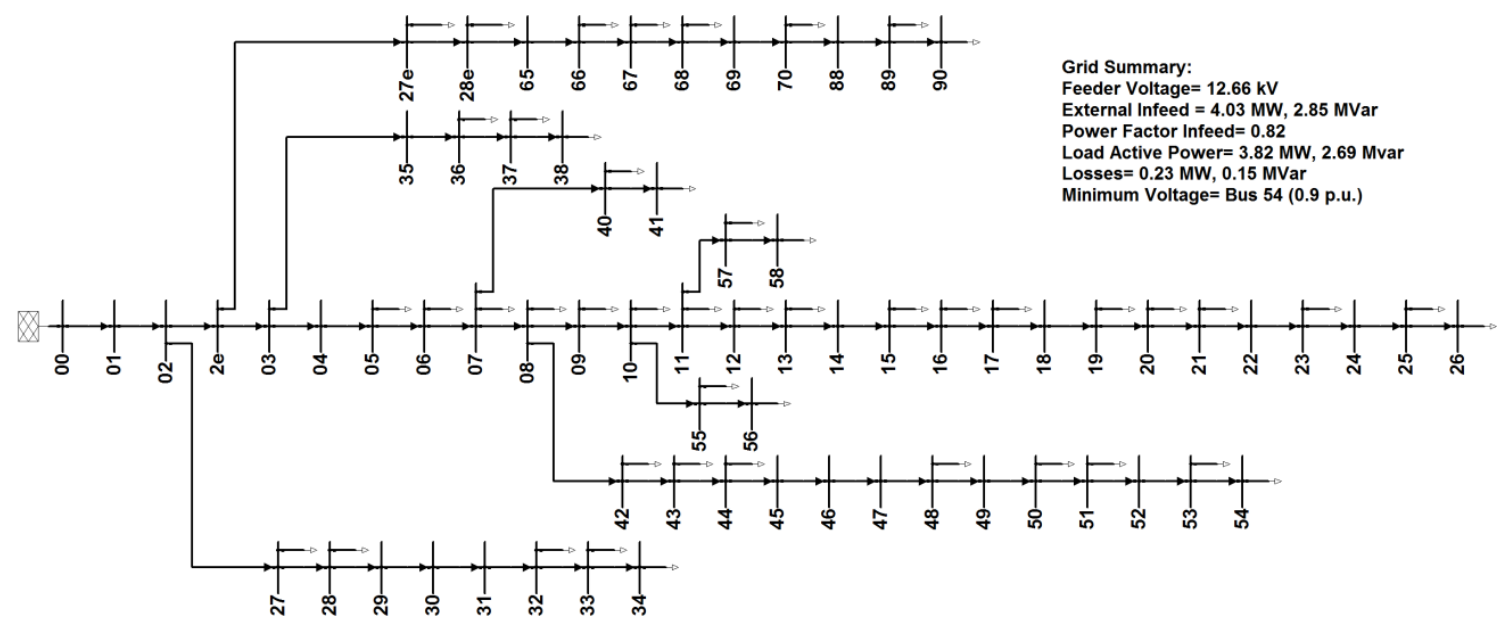

Figure 6.13 69-Bus Radial Distribution Test Feeder. 
feeder voltage is $12.66 \mathrm{kV}$ and the total load in the base case is $3.82 \mathrm{MW}$ and $2.85 \mathrm{MVar}$. Load flow calculations in the base case present 4.03 MW and 2.85 MVar power infeed from the external grid and a minimum voltage of 0.9 p.u. at bus-54. The network loss is $0.23 \mathrm{MW}$ or $5.7 \%$ of the total system active power. In order to study the behavior of the developed algorithm under the daily load characteristic we defined typical daily load curves for summer and winter, which are obtained from the Florida Electric Utility as shown in figure 6.16. Because the vast majority of customers in Florida are residential, peak demand in the summer season begins to climb in the morning, peaks during the hottest part of the day (4:00 PM), and levels off as the evening approaches.

This usage pattern corresponds to the increase of loads due to air conditioning for residential customers. In the winter season, the usage pattern has two distinct peaks: a larger one $(8: 00 \mathrm{AM})$ in the mid-morning and a smaller one $(8: 00 \mathrm{PM})$ in the late evening, which correspond to residential heating loads. In order to hold a consistent comparison of the cases under study, it is assumed that the normalized summer and

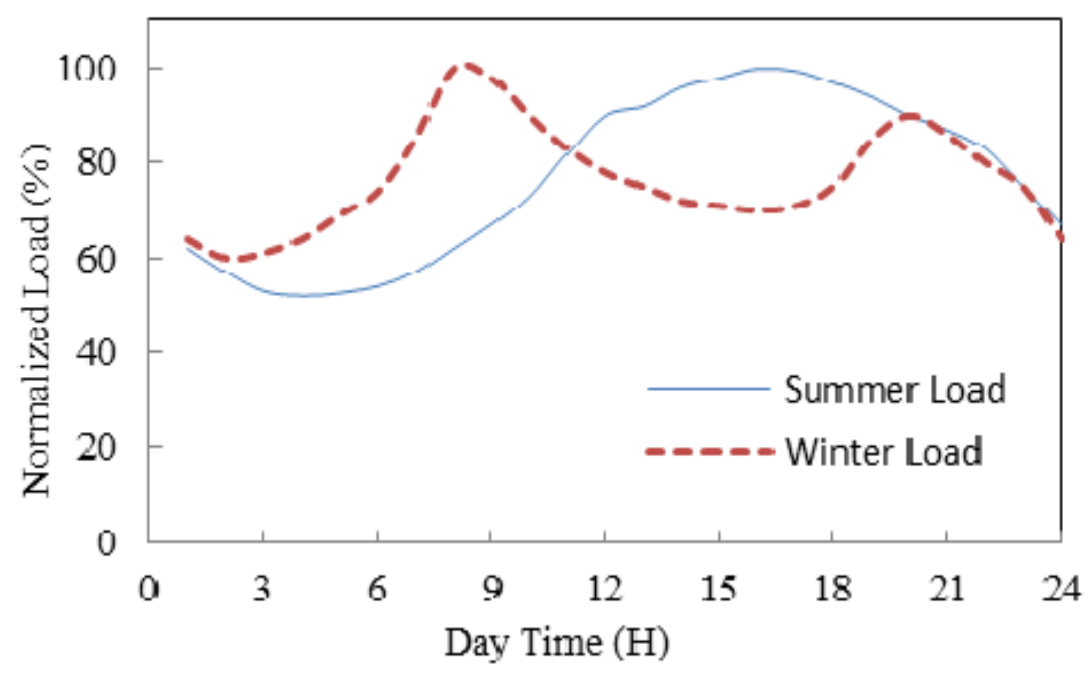

Figure 6.14 Florida's normalized summer and winter daily load curves 
winter curves have the same daily peak and the same daily energy consumption (kWh).

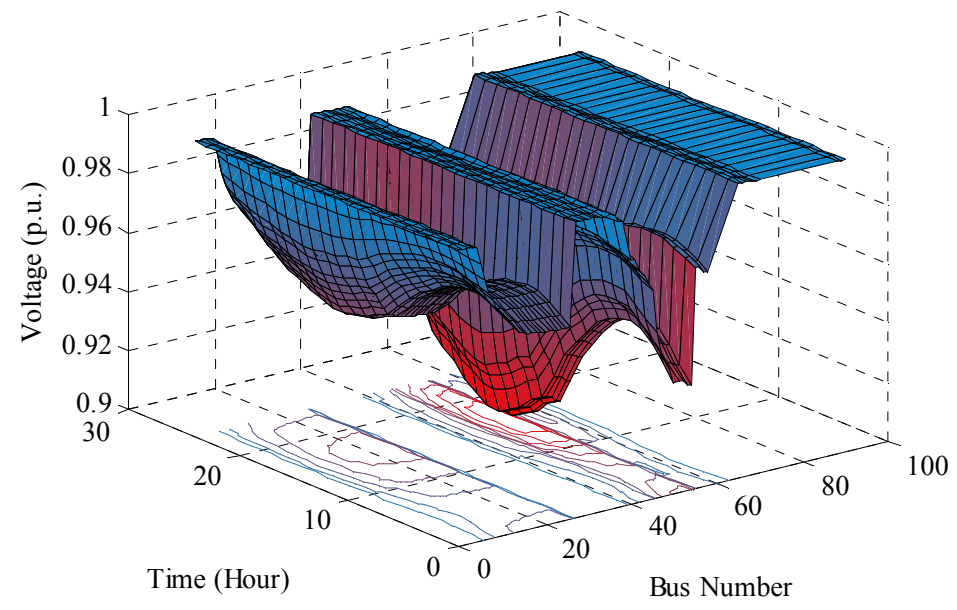

(a)

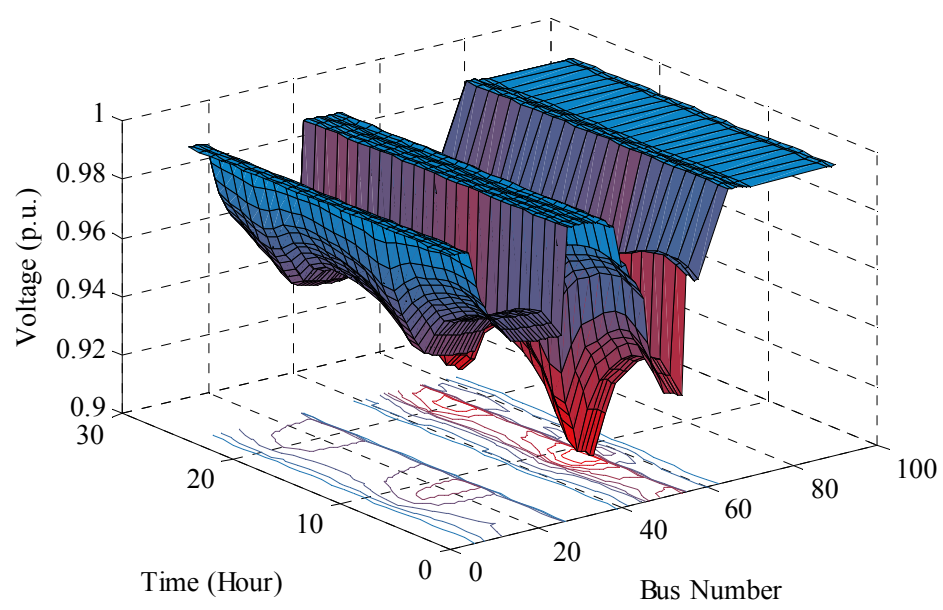

(b)

Figure 6.15 (a) 69-Bus daily voltage profile with no PHEVs for summer load (b) 69-Bus daily voltage profile with no PHEVs for winter load.

Therefore, we made a small change in winter curve to have the same integration as the summer curve during a 24-hour day. The PHEVs' daily consumption in both cases is intended to be $11.453 \mathrm{MWh}$. This amount of load is $15.5 \%$ of the total load before adding PHEVs (73.78 MWh). Figures 6.17 (a) and (b) show the voltage profile in all buses for 24 hours. The minimum voltage of the summer load happens at bus-54 at 4:12 
PM. Similarly, the minimum voltage of the winter load occurs at the same bus, but at 8:24 AM. The real time energy management algorithm was implemented in different cases with different techniques as follows:

\section{Case A. All PHEVs are connected to bus-20}

In this case all of the PHEVs are connected as an integrated car park to bus-20. Without any optimization this lumped load has the daily curve as shown in figure 6.18 with a solid line.

The daily peak is $3.383 \mathrm{MW}$ which occurs at 9:10 AM. Without any energy management procedure some buses at the ends of the feeders experience high voltage drop, i.e. 0.751 p.u. with summer load and 0.72 p.u. with winter load characteristics, which are shown in figure 6.19 and figure 6.20. This drop may harm the sensitive loads on the distribution feeder and needs to be improved by using the developed real-time energy management algorithm. In this section we used the optimization procedure described in section IV. The daily active power consumption of PHEVs is show in figure

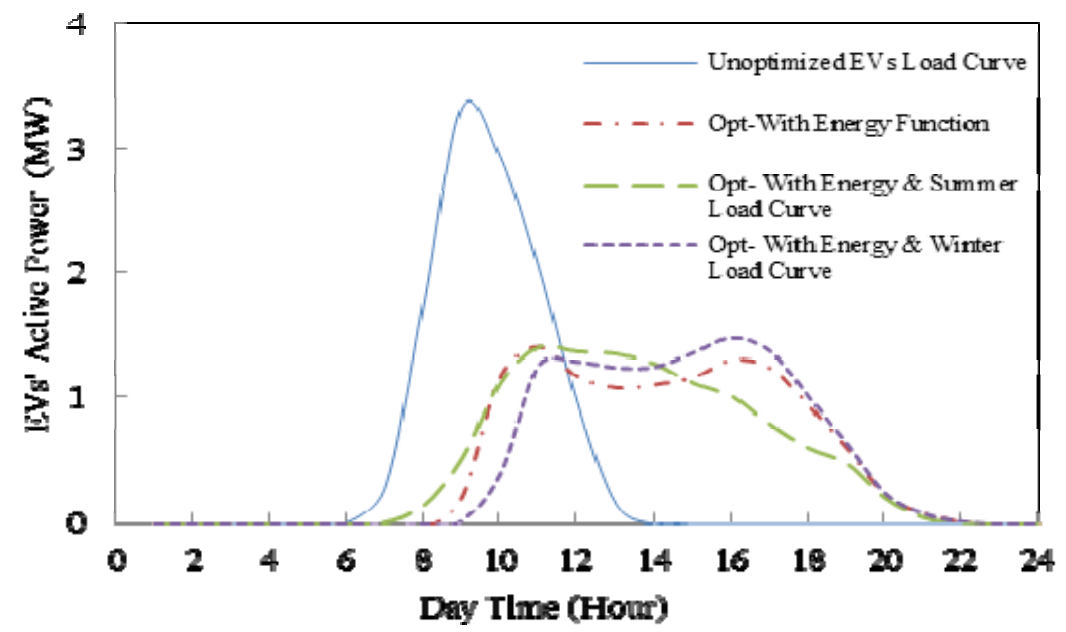

Figure 6.16 PHEVs daily load profile in with no optimization and different optimization objectives 
6.18 with a Dash-Dot curve. As can be seen, the PHEVs' consumption is distributed in a long hourly-based manner and the peak of the load is decreased considerably and shifted to 11:00 AM. The voltage daily profiles are also shown for summer and winter loads in figure 6.22 and 6.23 respectively, which present better voltage behavior during 24-hour operation. Not only voltage is affected by using the developed real-time energy management algorithm, but also the feeder losses decreased from $6.89 \%$ to $5.66 \%$ in summer and from $7.15 \%$ to $5.33 \%$ in winter load.

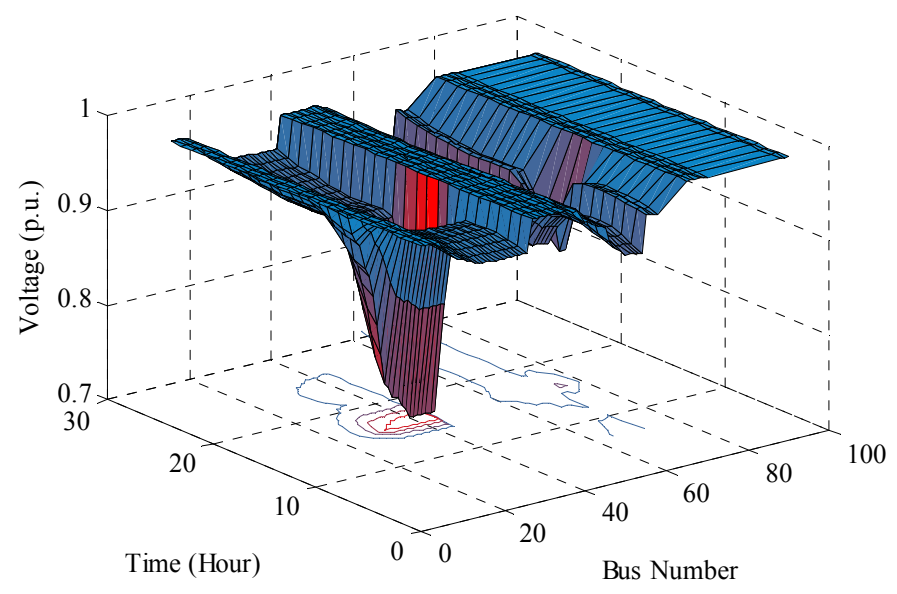

Figure 6.19 Daily voltage profile without real-time energy management algorithm for summer load.

Since the winter power losses improved more than summer losses, the results show that the performance of the real-time energy management algorithm is dependent on the feeder load curves as well. Therefore, the load curves of summer and winter loads of this feeder have been included in the real-time energy management algorithm as described previously. The results of the real-time energy management algorithm are also demonstrated in figure 6.18 with consideration of the summer and winter load characteristics in the energy management process. As shown in figure 6.16 the peak load 
of summer occurs at 4:00 PM. Therefore, by considering the load curve, the real-time energy management algorithm tries to put less loading stress around this time which is obvious in figure 6.18 as a long-dash curve. Accordingly, for the winter load curve with two peaks, the main one at 8:00 $\mathrm{AM}$ and minor one at 8:00 PM, the real-time energy management algorithm tends to charge the PHEVs after the first peak and before the second peak. The daily voltage profiles improved in these cases and are similar to figures 6.21 and 6.22 .

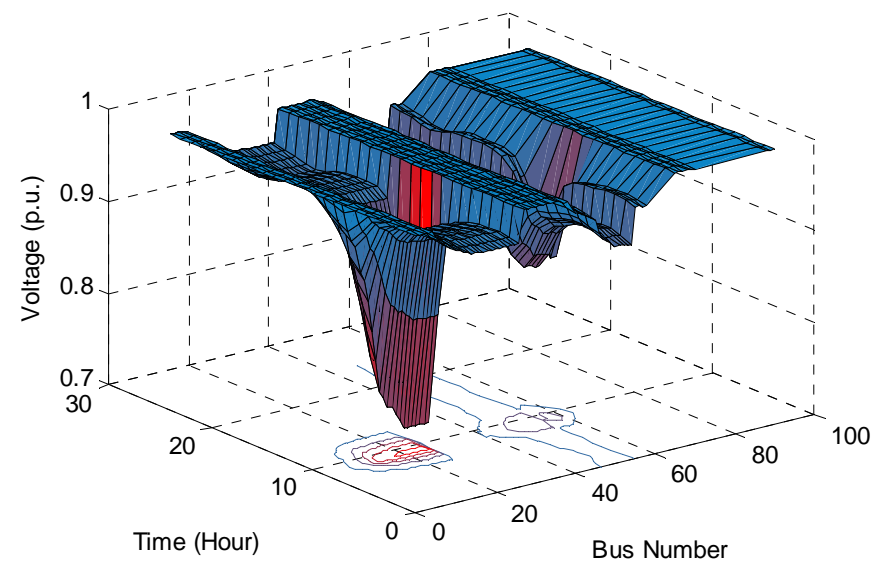

Figure 6.17 Daily voltage profile without real-time energy management algorithm for winter load.

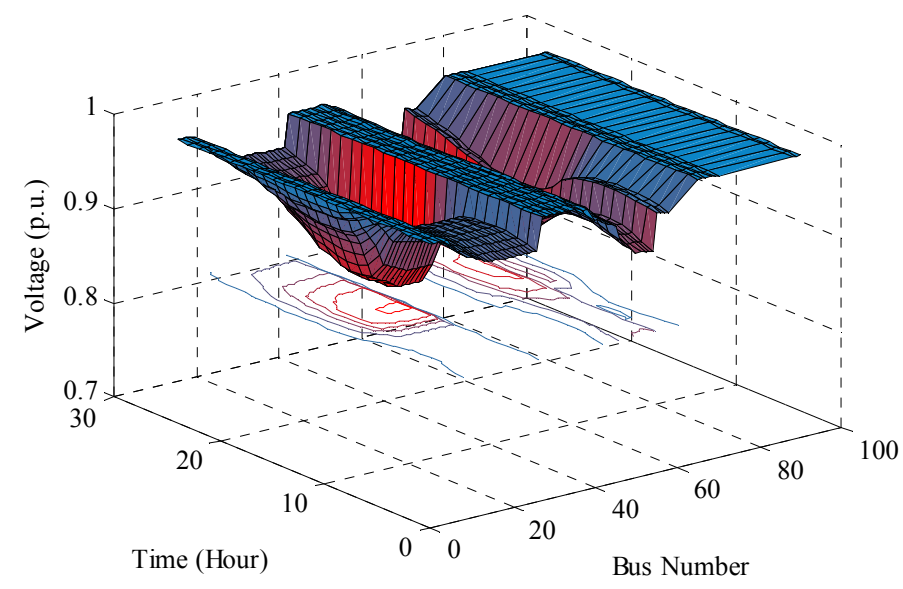

Figure 6.18 Daily voltage profile with real-time energy management algorithm for summer load. 


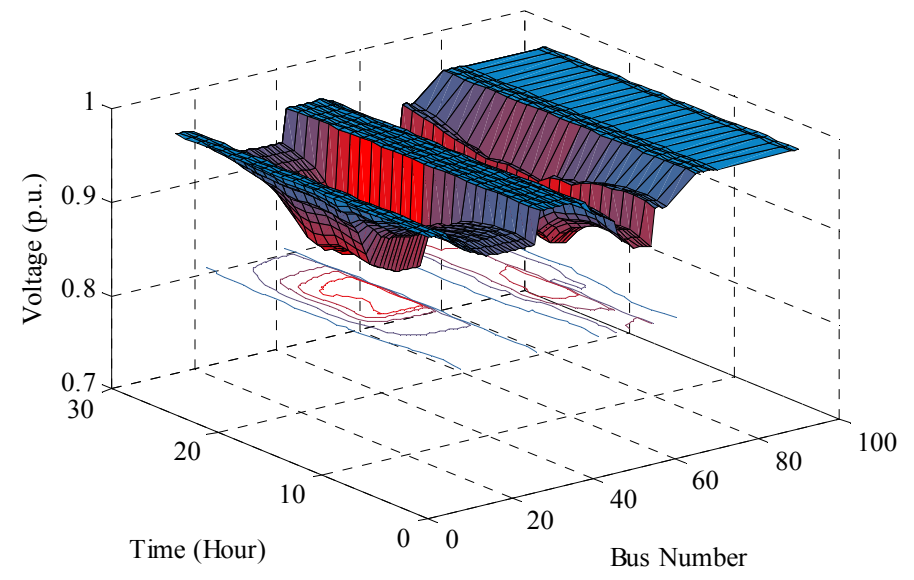

Figure 6.19 Daily voltage profile with real-time energy management algorithm for winter load.

\section{Case B. All PHEVs are distributed equally to 5 buses}

In order to study the effect of the PHEVs' load distribution in a radial distribution feeder, all the PHEVs were distributed equally at buses 20, 30, 41, 48 and 67.

Therefore, the same amount of load which was considered in the previous case is

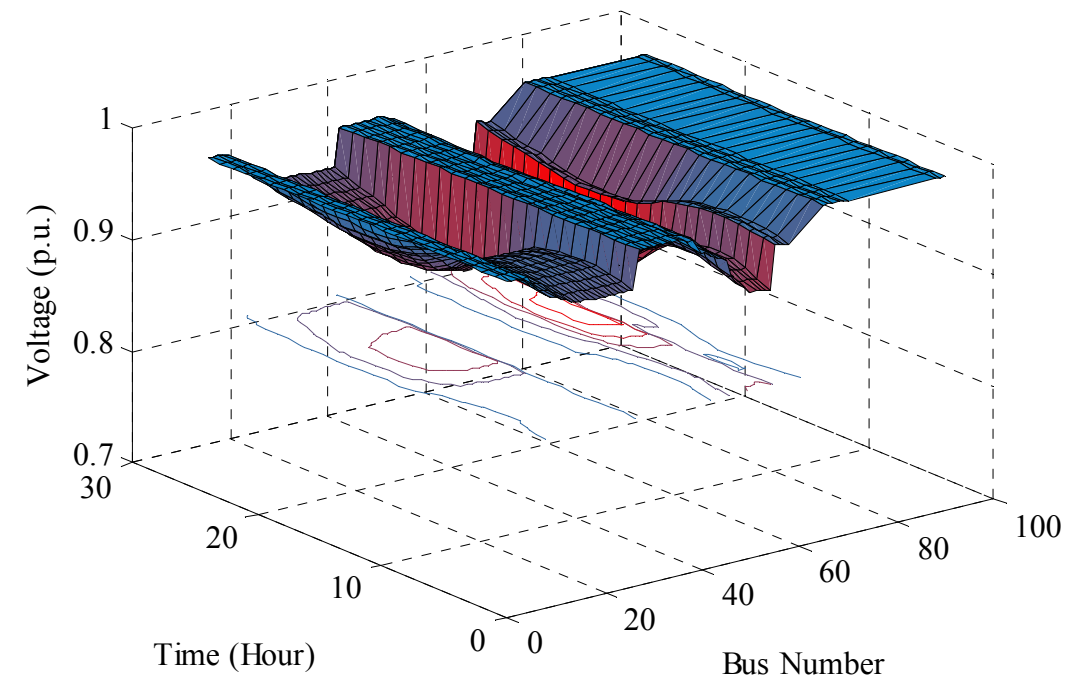

Figure 6.20 Daily voltage profile with real-time energy management algorithm considering energy function and summer load curve 


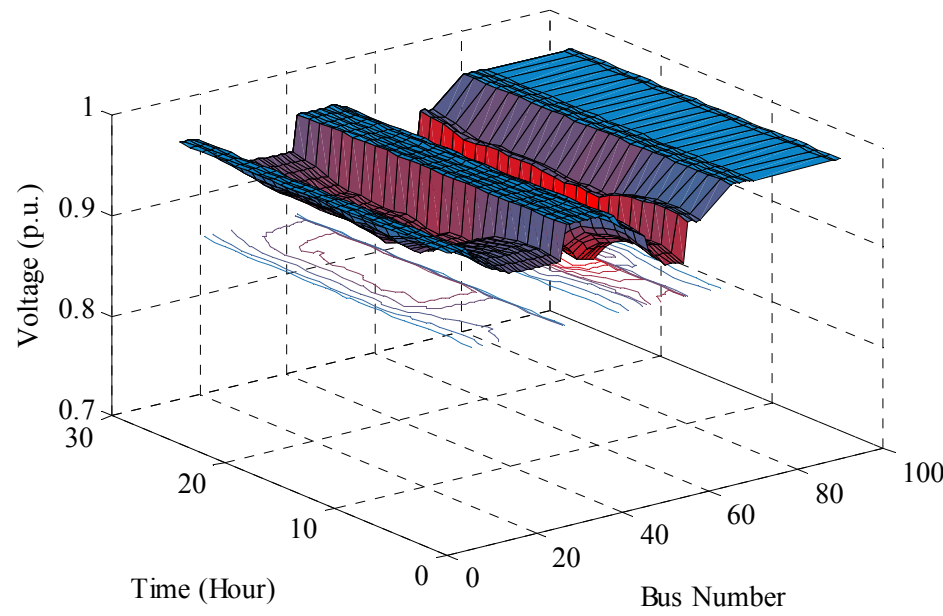

Figure 6.21 Daily voltage profile with real-time energy management algorithm considering energy function and winter load curve.

equally distributed at these nodes and the real-time energy management algorithm uses the PHEVs' same daily distribution curve and feeder total daily load curve to manage the PHEVs charging process. The results with the real-time energy management algorithm are similar to the previous cases for the summer and winter optimized daily load curves. The 24-hour daily load-flow results in better voltage profiles, which are shown in figure 6.23 and 6.24 for summer and winter loads, respectively.

The real-time energy management algorithm objective function is based on energy by considering the feeder load characteristics. The voltage profiles in this case are similar to the feeder main profiles before connecting PHEVs, i.e. Figure 6.17 (a) and (b). Table 6.5 presents a summary of the results for all cases studied in this chapter. Obviously, the feeder total losses and voltage profile improved in this case as well. For example, in the summer load case, the feeder total loss decreased from $5.59 \%$ to $4.53 \%$.

\section{Case C. All PHEVs are distributed equally to 10 buses}

In this last case, all the PHEVs are distributed equally among ten buses in order to study the PHEVs' parking distribution effect in the radial feeder. These nodes are: 20, 26, 


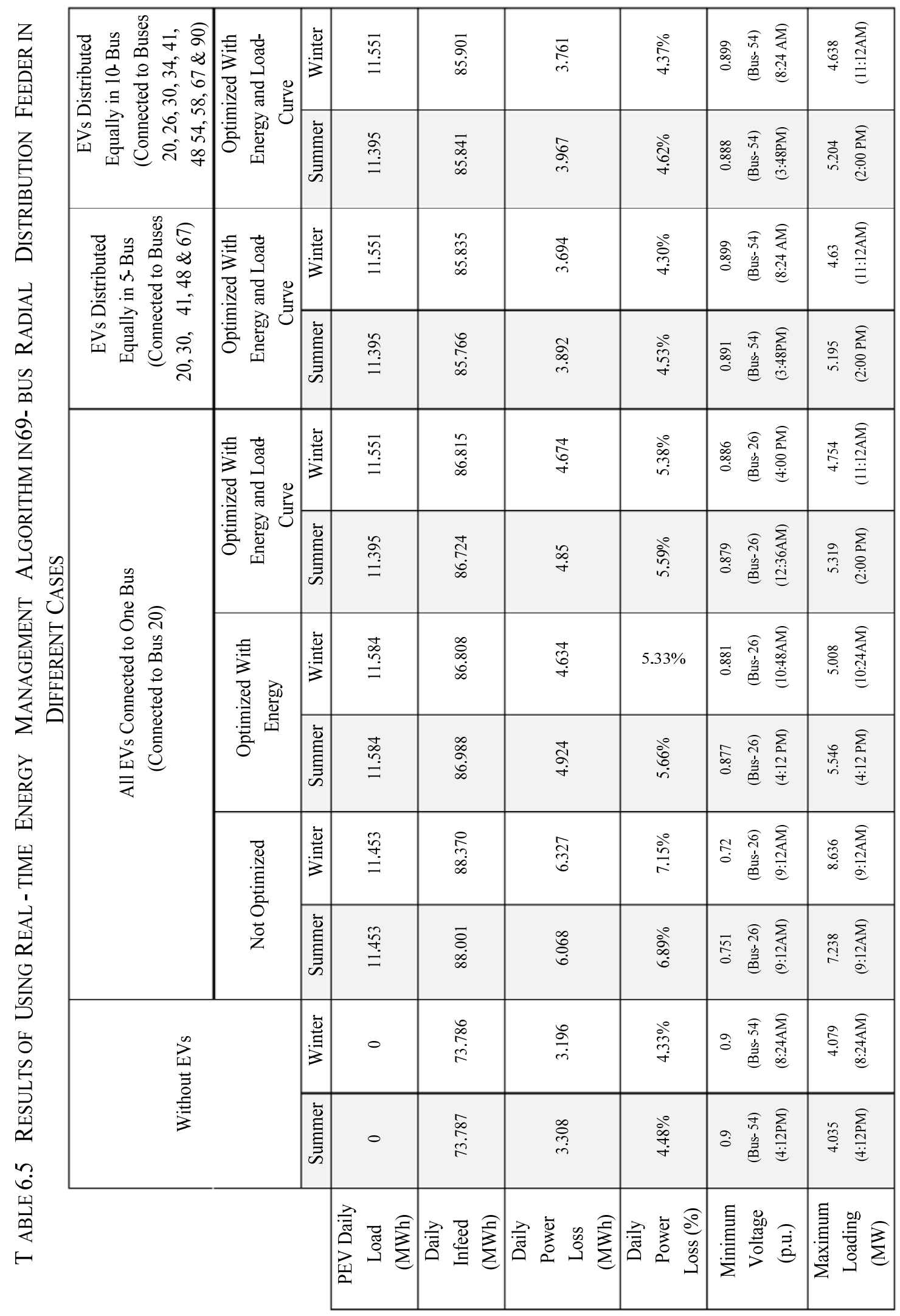


$30,34,41,48,54,58,67$ and 90 . Similar to the previous case, the real-time energy management algorithm will improve the system voltage profiles. The response will be even more similar to the previous case and is comparable to the case with no PHEVs. The results of minimum voltage, total loss of feeder, and its maximum loading and peak hours are also presented at Table 6.5. They illustrate that increased distribution of PHEVs no longer has a significant effect on radial distribution system parameters.

\subsection{Conclusion}

In this section, a real-time energy management algorithm for PHEVs charging in grid-connected smart charging parks based on charging priority levels was developed. The developed algorithm allows $\mathrm{V} 2 \mathrm{G}$ and $\mathrm{V} 2 \mathrm{~V}$ functionalities and aims at minimizing the total cost of charging by handling the charging rates of the PHEVs. An advantage of the developed algorithm is that the charging rates of the PHEVs during their parking period are varying according to their state of charge. A Fuzzy agent was used as a component within the developed algorithm. Energy tariff, load demand and PV output power profiles are elements within the algorithm. The performance of the developed algorithm was tested by simulating its implementation on a charging park connected to the IEEE standard 69-bus system at different penetration and distribution levels. The results show a reduction in the overall cost of charging as well as a significant improvement in the voltage profile and the losses in the system. This algorithm is easy to develop and implement because it is not based on an optimization technique, allowing several objectives to be targeted simultaneously. 
7. Economic Analysis of Real-time Large Scale PEV Network Power Flow Control Algorithm with the Consideration of V2G Services

Like the plug-in hybrid electric vehicles (PHEVs,) plug-in electric vehicles (PEVs) also have battery banks and can be charged by connecting to the utility grid. But PEVs derive all their power from their battery packs and thus have no internal combustion engines, fuel cells, or fuel tanks. Therefore, compared with PHEVs, PEVs are cleaner with almost zero greenhouse gas emissions. Because of only depending on electric power, PEV battery banks capacities are usually much larger than PHEV battery bank capacities. For example, Tesla Model S PEVs are equipped with an $85 \mathrm{kWh}$ battery pack, and the 2015 Nissan Leaf PEVs are equipped with a $24 \mathrm{kWh}$ battery pack. Therefore, without proper control, PEVs can bring more serious impact to the utility grid compared with PHEVs. On the other hand, if smartly regulated, a PEV network with several PEVs can provide more powerful vehicle to grid services.

\subsection{Introduction}

PEVs are gaining much popularity due to the global call for clean energy. PEVs are expected to have a major impact in the auto industry given advantages in city pollution, less dependence on oil, and expected rise in overall transportation efficiency. World governments are pledging billions to fund development of PEVs and their components. The U.S. has pledged US\$2.4 billion in federal grants for PEVs and batteries. China has announced it will provide US\$15 billion to initiate an electric car 
industry. Based on the annual energy review report from the U.S. Energy Information Administration, in 2011 more than $80 \%$ of the energy consumed in the USA was generated by petroleum, natural gas, and coal. Renewable energy sources supplied no more than $8 \%$ of the total energy. At the same time, transportation consumed $28 \%$ of the total energy [104]. Therefore, the use of renewable energy to charge PEVs will be an attractive choice, particularly if customers would pay less by driving PEVs and gain benefits by involving their PEVs in the V2G services such as grid frequency regulation and reactive power support [145].

Recently, several researchers have proposed ideas and models that are related to PEV charging optimization and economic analysis of V2G services. In [84], [136] and [146], load management solutions for coordinating the charging process of multiple PEVs in a smart grid system based on real-time minimization of the total cost of generating the energy plus the associated grid energy losses were proposed. However, they did not consider the inclusion of a renewable energy source in the system. This holds the implementation of these algorithms back since the concept of PEVs is linked to obtaining the power to charge them from renewable energy [130]. In addition, the control strategy considered charging priority level, but the level is based on how much the owner of the PEV is willing to pay, not the state of charge (SOC) of the PEVs' batteries. In [78] and [81], intelligent methods for scheduling the use of available energy storage capacity from PEVs are proposed. The batteries on these PEVs can either support power to the grid when parked, known as the V2G concept, or absorb power from the grid. However, the details about the energy dispatch during charging and V2G process were not given. Also, the SOCs of the PEVs' batteries were not considered during the process. In [59], a fuzzy 
logic controller was used to optimize the charging process of a small group of PEVs in a car park infrastructure. V2G and V2V services are realized, but the economic analysis of the proposed model was not given. Also, the renewable energy farm scale is not optimized. Therefore, it is left unclear whether joining the V2G will bring benefits to the customers or not. In [147] and [148], economic assessments on V2G frequency regulation with the estimation of achievable power capacity from PEVs for V2G frequency regulation and battery degradation were studied based on four different penalty cases. However, those models only considered a system with 1000 PEVs, and the charging of PEVs only considered the achievable power capacity and contracted power capacity for frequency regulation. The real charging process was not simulated. Therefore, the proposed model can't guarantee that the SOCs of the PEVs' batteries will be above a certain level at the next departure time, which may influence the customer's driving behaviors.

In this chpter, the economic analysis of a real-time large scale PEV network power flow control algorithm for charging impact limitation and V2G services was studied. An optimization process for a renewable energy farm with both solar and wind energy by using a genetic algorithm (GA) is given. The studies included both the feasibility and economic analysis. This chapter is organized as follows: in Section 7.2, the large scale PEV network potential application for grid secondary frequency regulation is analyzed. Section 7.3 gives the overall description of the large scale PEV network model. Section 7.4 analyzes the large scale PEV network energy consumption. The analysis of annual local load pattern, renewable energy resources pattern, and grid frequency regulation signal together with the methodology of optimal renewable energy farm design 
for charging the PEV network is given in Section 7.5. Section 7.6 presents the fuzzy logic based smart charging algorithm for PEV networks with the consideration of maximum utilization of renewable energy resources and regulating the grid frequency. The overall system economic analysis model is described in Section 7.7. Section 7.8 gives the simulation results of a case of study of a 50,000 PEV network in the South Florida power system and analyzes the economic effects. Finally, a conclusion of this chapter is presented in Section 7.9.

\subsection{Large Scale PEV Network V2G secondary control potentials}

Secondary control typically includes the balancing services deployed in the "minutes" time frame. This control is accomplished using the Balancing Authority's control computer and the manual actions taken by the dispatcher to provide additional adjustments. Secondary control also includes initial reserve deployment for disturbances. Secondary control maintains the minute-to-minute balance throughout the day and is used to restore frequency to its scheduled value, usually $60 \mathrm{~Hz}$, following a disturbance.

Secondary control is provided by both spinning and non-spinning reserves. The most common means of exercising secondary control is through Automatic Generation Control (AGC). AGC operates in conjunction with Supervisory Control and Data Acquisition (SCADA) systems. SCADA gathers information about an electric system, in particular system frequency, generator outputs, and actual interchange between the system and adjacent systems. Using system frequency and net actual interchange, plus knowledge of net scheduled interchange, it is possible to determine the system's energy balance with its interconnection in near-real-time. AGC computes an area's Area Control Error (ACE) 
from interchange and frequency data. ACE tells whether a system is in balance or needs to make adjustments to generation. AGC software, while observing ACE, automatically determines the most economical output for generating resources while observing energy balance and frequency control, usually by sending set points to generators. Some generators also use pulse-accumulator methodology to derive a set point from pulses sent by AGC, but these have become less common over time.

\section{Secondary Control Reserve}

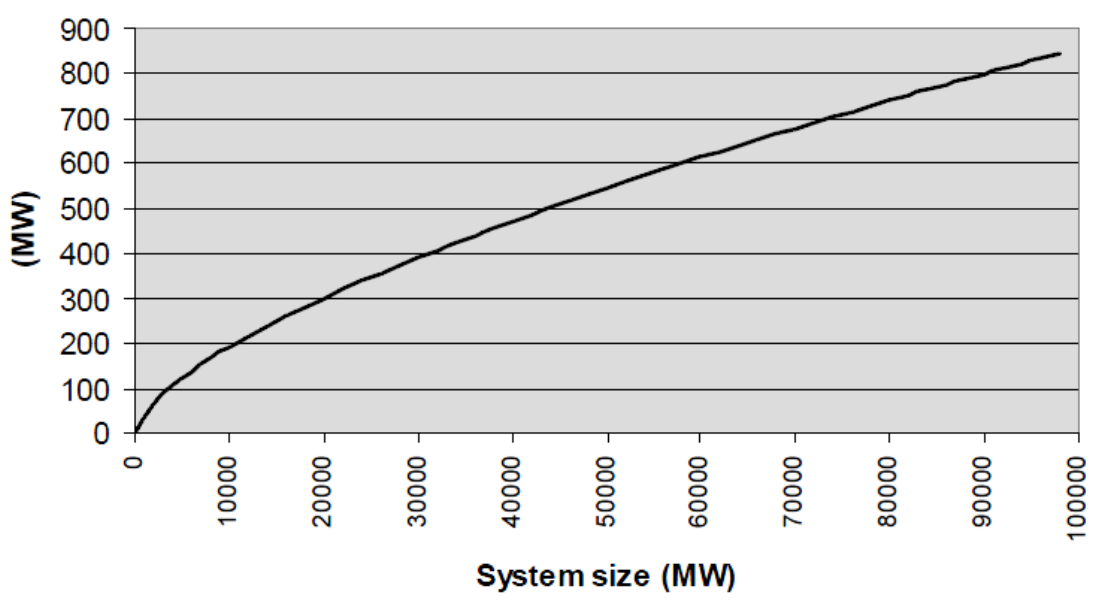

Figure 7.1 Secondary control power reserve VS system power.

Secondary control should have a response timeframe of 30s-10minutes, and the relationship between the secondary control reserve capacity and the system capacity is shown in figure. 7.1.

For a PEV network with a large number of PEVs in a big area like South Florida, if the PEVs charging power requirement and SOCs are monitored by a central aggregator which plays the role of the SCADA system, then the PEV network can be involved in the secondary control reserves for the utility grid. Compared with conventional load, PEVs can not only dramatically reduce the charging power in a very short time, but also can act as 
energy sources that inject energy to the grid. Therefore, PEV networks can play an important role in the future large area utility grid frequency secondary control.

\subsection{Large Scale PEV Network Model}

A diagram of the system under study is as shown in figure 7.2. The PEVs can be connected to the utility grid through power electronic chargers on both the AC and DC sides. The diagram of a hybrid AC-DC power system involving renewable energy sources and a PEV network is shown in figure 7.3. It consists of several power plants, a local load, a PEV network with a certain number of PEVs, and a renewable energy farm with both wind turbines and PV panels that can supply enough energy for the PEV network. Also, the system has a central control aggregator which acts as the SCADA system and uses the collected next period forecasting information such as the renewable energy farm output power, the local load, and the total power needed by the PEV network, together with the frequency regulation information to manage PEV charging, regulate the frequency of the utility grid, and balance the power flow through wireless communication in this area. The PEVs are all connected to the grid through a level-2 charging station, either in public areas or at home. To minimize the air pollution, the energy consumed by the charging of PEVs should be supplied by the renewable energy farm. Additionally, a smart charging algorithm was designed to manage power flow in order to limit the impact PEVs have on the utility grid during the charging process and increase revenue through $\mathrm{V} 2 \mathrm{G}$ services. To fulfill all of these requirements, high accurate PEV power requirement stochastic model, unit renewable energy sources output power analysis model, and local load forecasting model are needed. Furthermore, discharging a 
PEV's battery during the V2G service degrades both its lifetime and the capacity. Therefore, an economic analysis model is needed to estimate the daily and annual revenues in order to verify that the income compensates for the battery's degradation costs.

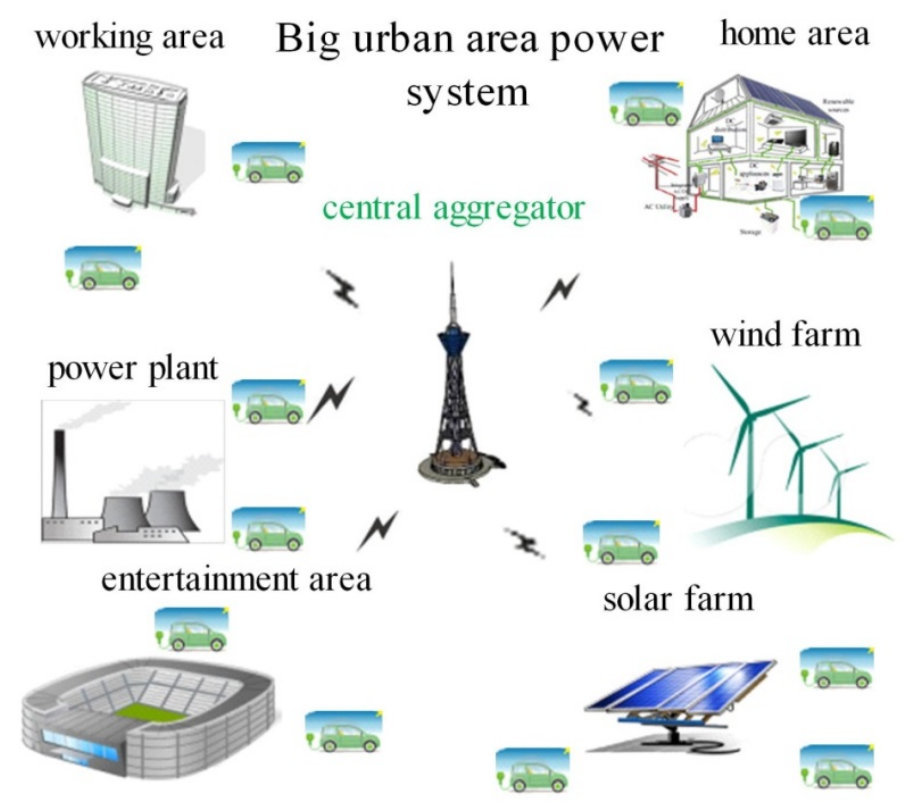

Figure 7.2 Overview of the proposed PEVs network charging system with renewable resources

\subsection{Analysis of Large Scale PEV Network Energy Consumption}

The PEV network model contains a certain amount of PEVs with different sizes, battery capacities, and energy consumptions per mile. The specific detail is shown in Table 7.1. Whenever a PEV is connected to the grid, the owner is requested to set the next departure time, and the system will make a record. At the departure time, the SOC of the battery is expected to be above $80 \%$. Therefore, during the following period before the departure time, these PEVs can either be charged or discharged based on the grid's power flow, renewable energy sources output power, and frequency regulation 
Table 7.1 Parameters for PEVs In Different Size

\begin{tabular}{|c|c|c|c|}
\hline PHEVs model & Percentage & Battery capacity (kWh) & $\begin{array}{l}\text { Energy consumption } \\
\text { per mile }(\mathrm{kWh} / \text { mile })\end{array}$ \\
\hline compact sedan & $32.5 \%$ & $20-35$ & 0.2 \\
\hline full-size sedan & $37.5 \%$ & $40-65$ & 0.3 \\
\hline $\begin{array}{l}\text { mid-size SUV } \\
\text { or pickup }\end{array}$ & $20 \%$ & $55-80$ & 0.45 \\
\hline $\begin{array}{l}\text { full-size SUV } \\
\text { or pickup }\end{array}$ & $10 \%$ & $75-110$ & 0.6 \\
\hline
\end{tabular}

requirement. For protection reasons, during the $\mathrm{V} 2 \mathrm{G}$ service, the SOC of the batteries shouldn't go below $20 \%$ [14].

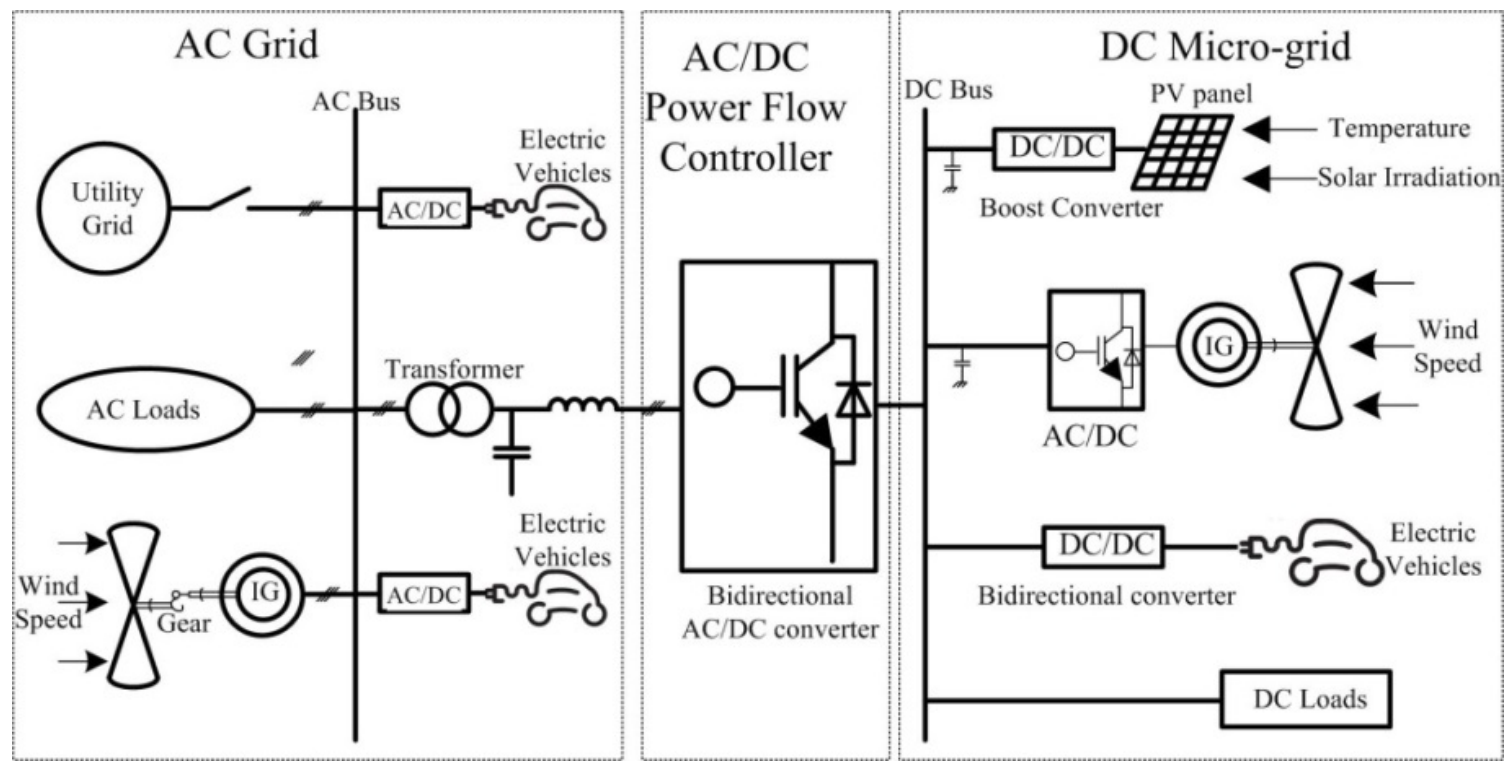

Figure 7.3 PEVs charging hybrid AC-DC power system

The estimated power demanded by each individual PEV $\left(\hat{p}_{P E V, i}\right)$ can be represented by equations (7.1) and (7.2), respectively during the daytime and nighttime.

$$
\begin{gathered}
\hat{P}_{P E V, i}=\frac{\hat{M}_{d} \times E_{m}}{2\left(\hat{D} w_{t}-\hat{A} w_{t}\right)} \\
\hat{P}_{P E V, i}=\frac{\hat{M}_{d} \times E_{m}}{2\left(\hat{D} h_{t}-\hat{A} h_{t}\right)}
\end{gathered}
$$


where

$\hat{A} h_{t} \quad$ is the estimated home arrival time, PEVs are connected to the grid,

$\hat{A} w_{t} \quad$ is the estimated workplace arrival time, PEVs are connected to the grid,

$\hat{D} h_{t} \quad$ is the estimated home departure time, PEVs are disconnected from the grid,

$\hat{D} w_{t} \quad$ is the estimated workplace departure time, PEVs are disconnected from the grid,

$E_{m} \quad$ is the energy efficiency for the PEV (miles $/ \mathrm{kWh}$ ),

$\hat{M}_{d} \quad$ is the estimated number of miles driven daily (mile),

$\hat{P}_{P E V, i} \quad$ is the estimated $i^{\text {th }} \mathrm{PEV}$ power demanded $(\mathrm{kW})$.

To obtain the stochastic model of $\hat{P}_{P E V, i}$, the distribution functions of $\hat{M}_{d}, \hat{D} h_{t}$, $\hat{D} w_{t}, \hat{A} h_{t}$ and $\hat{A} w_{t}$ should be found first. Based on driving pattern statistics in the USA, the average yearly total miles driven in USA is 12,000 miles with $50 \%$ of drivers driving 25 miles or less per day, and $80 \%$ of drivers driving 40 miles or less per day [149]. Therefore, a $\log$ normal distribution with $\mu_{m}=3.37, \sigma_{m}=0.5$ was selected to approximate the probability density function (PDF) of $M_{d}$. Also, based on the Central Limit Theorem (the conditions under which the mean of a sufficiently large number of independent random variables, each with finite mean and variance, will approximately be normally distributed [144]) the departure time from home to the work place and from the

Table 7.2 Arrival And Departure Times Distribution Parameters

\begin{tabular}{ccccc}
\hline \hline & \multicolumn{2}{c}{ Arrival } & \multicolumn{2}{c}{ Departure } \\
\hline Parameter & $\hat{A} h_{t}$ & $\hat{A} w_{t}$ & $\hat{D} h_{t}$ & $\hat{D} w_{t}$ \\
$\mu_{T}[h]$ & 18.5 & 9 & 8.5 & 17.5 \\
$\left(\sigma_{T}\right)^{2}[h]$ & 1.2 & 1.5 & 1.2 & 1.5 \\
\hline \hline
\end{tabular}


workplace back home obey normal distribution. The parameters are shown in Table 7.2.

With the PDF of daily duration time and the PDF of daily travel distance, the power consumptions of each class of PEVs was computed using the statistical distribution fitting toolbox in Matlab and Monte Carlo simulation. With 50,000 samples, the PDF of constant power needed by each PEV when it is connected to the grid, $\hat{P}_{P E V}$, was found as an inverse Gaussian distribution with $\mu_{p}=1.573$ and $\lambda_{p}=3.652$. The distribution function for $\hat{P}_{P E V}$ is given in equation (7.3).

$$
f_{X}\left(x, \mu_{p}, \lambda_{p}\right)=\sqrt{\frac{\lambda_{p}}{2 \pi x^{3}}} \exp \left\{-\frac{\lambda_{p}}{2 \mu_{p}{ }^{2} x}\left(x-\mu_{p}\right)^{2}\right\}
$$

Following the evaluation of the probability distribution function of $\hat{P}_{P E V}$, the forecasting model of the power needed by PEVs in this network system was built. For instance, at a certain time $t$, the SOCs of the PEVs already connected to the grid and their power requirements are already known. Therefore, in order to forecast the power needed by the PEVs which will be connected to the grid during the upcoming period $T$, the following equation (7.4) can be used.

$$
\hat{P}_{\text {upcoming }}=\int_{t}^{t+T} f_{A_{t}}\left(x, \mu_{A_{t}}, \sigma_{A_{t}}\right) d t \times N P \times \hat{p}_{P E V_{-} a v g},
$$

where

$f_{A_{t}}\left(x, \mu_{A_{t}}, \sigma_{A_{t}}\right)$ is the PDF of grid connecting time $A_{t}$,

NP is the total number of PEVs in the PEVs network,

$\hat{P}_{P E V_{-} \text {avg }} \quad$ is the average constant power requirement for all PEVs when they are connected to the parking lot. $\hat{P}_{P E V_{-} \text {avg }}$ can be calculated from the PDF of $\hat{P}_{P E V}$. 


\subsection{Analysis of Annual Local Load Pattern, Renewable Energy Resources Pattern, and Grid Frequency Regulation Signal}

\subsubsection{Local load pattern analysis}

The one year weekday South Florida load curve, summer load curve, and winter load curve are shown in figure 7.4 (a), (b) and (c). In summer, there is only one peak during the whole day, which happens around noon. This is due to the power consumption of air conditioners. In winter time, there are two peaks, one in the morning and one in the

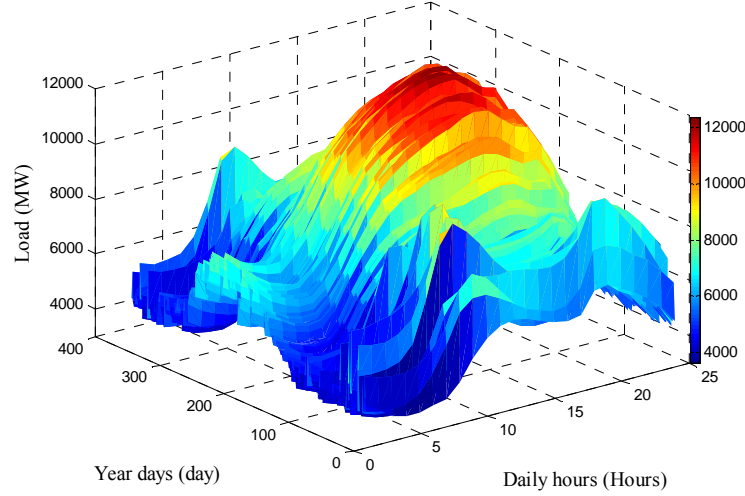

(a)

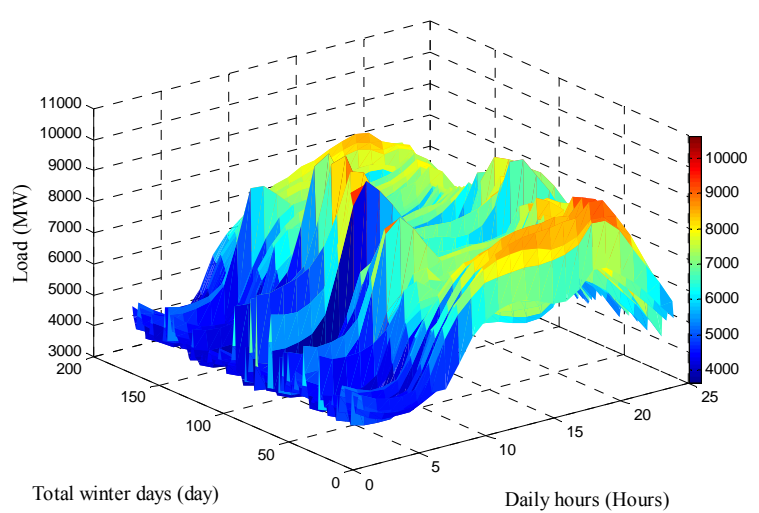

(c)

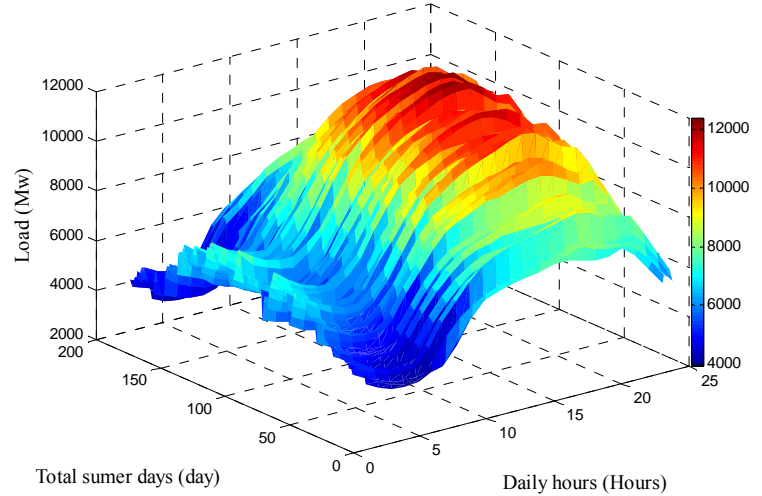

(b)

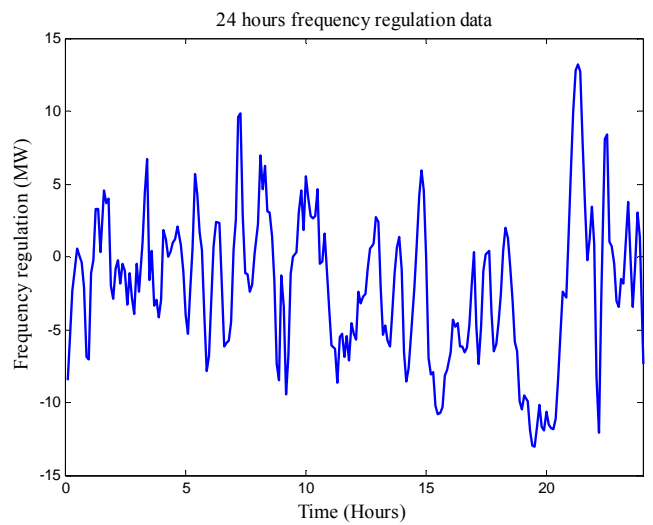

(d)

Figure 7.4 South Florida local load and frequency pattern: (a) load pattern of the year, (b) summer load pattern, (c) winter load pattern, (d) frequency regulation pattern. 
afternoon. These load peaks occur because of cooking in the household. Based on the data, back-propagation neural network (BPNN) was used to forecast the hourly load. Since this chapter is not focused on load forecasting, the details of BPNN model are not covered.

\subsubsection{Grid frequency regulation analysis}

The one day frequency regulation data from Pennsylvania-New Jersey-Maryland Interconnection (PJM) on July $1^{\text {st }} 2010$ is shown in figure 7.4 (d). The maximum power variation is $13 \mathrm{MW}$, around $1.1 \%$ of the forecasted load peak for the day. This value is appropriate for secondary frequency control regulation.



(a)

Average 24 hours wind speed during one year.

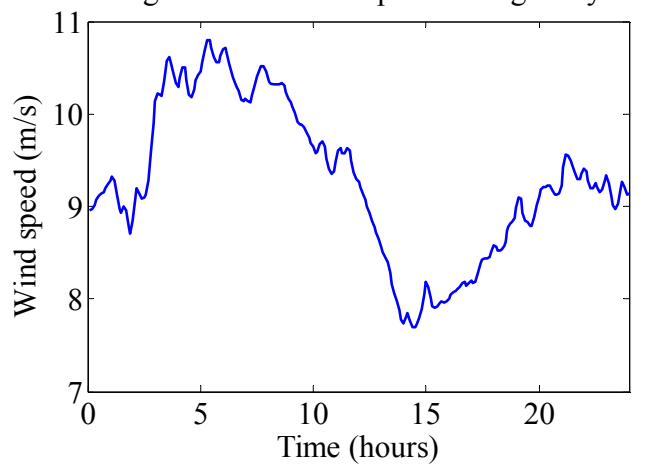

(c)

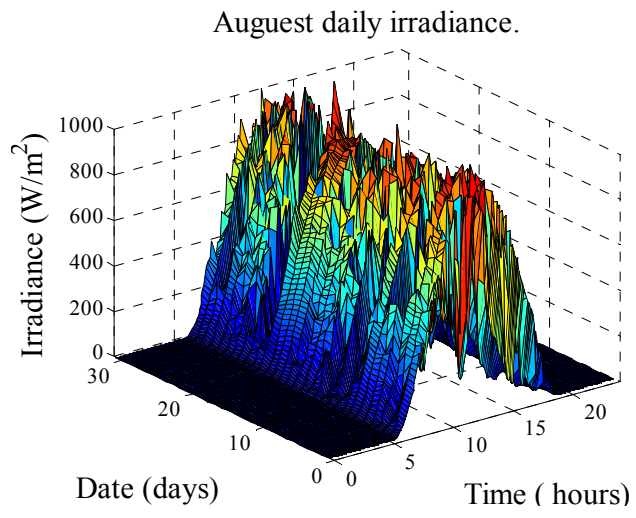

(b)

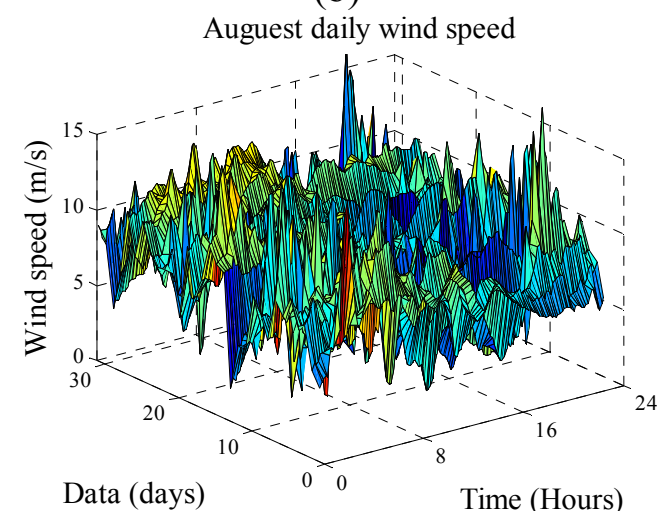

(d)

Figure 7.5 Historical wind data for one day and one month. 


\subsubsection{Renewable energy resources pattern analysis}

In order to optimize the size of the renewable energy farm to support enough power to the PEV network, historical solar irradiance and wind speed data are analyzed together with the daily PEV network energy consumption. In this chapter, the wind and solar data for the year 2007 in South Florida was used. The one year average daily solar irradiance data and daily solar irradiance data in August 2007 is shown in figure 7.5 (a)

and (b). The one year average wind speed data and daily wind speed data in August 2007 is shown in figure 7.5 (c) and (d).

The daily energy generated by the solar panels can be calculated by using equation (7.5), in which $A$ is the solar panel size, $u(t)$ is the solar irradiance and $\beta$ is the solar panel efficiency. In this model $\beta=22 \%$.

$$
E_{\text {sloar }}=A \times \beta \int_{t=0}^{t=24} u(t) \times t d t
$$

For the output of the wind farm, a General Electric 1.5-MW turbine model 1.5sle [150] is used to determine the output power generated by the wind under different wind speed situations. The total daily energy output from the wind farm is calculated in (7.6). Where $N$ stands for the number of wind turbines, $S_{(t)}$ is the wind speed at time $t$ and $f_{\text {wind }}\left(S_{(t)}\right)$ represents the single turbine output power at the time $t$.

$$
E_{\text {wind }}=N \times \int_{t=0}^{t=24} f_{\text {wind }}\left(s_{(t)}\right) \times t d t
$$

\subsubsection{Optimal renewable energy farm design for the PEV network}

In order to build a renewable energy farm with an energy output that matches the energy consumption of the PEV network as closely as possible, the cost function regarding the size of the renewable energy farm is given in equation (7.7). 


$$
\begin{aligned}
& f(A, N)= \\
& \sum_{d=1}^{d=365}\left|A \times \beta \int_{t=0}^{t=24} u(t) \times t d t+N \times \int_{t=0}^{t=24} f\left(s_{(t)}\right) \times t d t \times t d t-E_{P E V S}(d)\right|
\end{aligned}
$$

The best solution is based on a proper $A$ and $N$ that can minimize the cost function (7.7). A Genetic Algorithm (GA) was selected to find the best solution with the population size set as 20 , crossover rate set as 0.8 , and mutation rate set as 0.02 .

The optimization process is shown in figure 7.6. The optimized renewable farm should contain 191919.767 square meters of solar panels and 15 units of General Electric 1.5-MW turbine model 1.5sle to generate enough energy for the PEVs daily consumption.

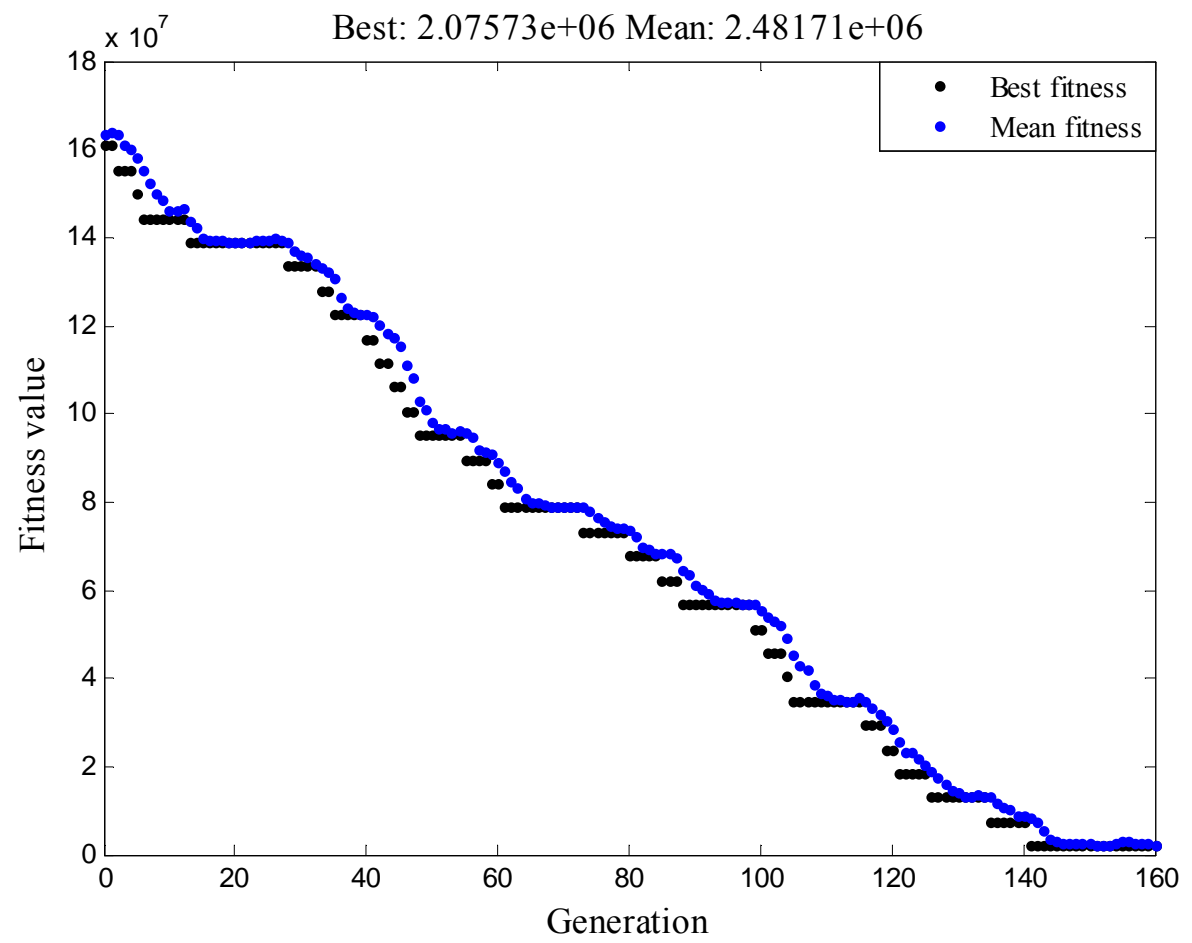

Figure 7.6 Renewable energy farm scale optimization process for PEV network charging by using GA. 


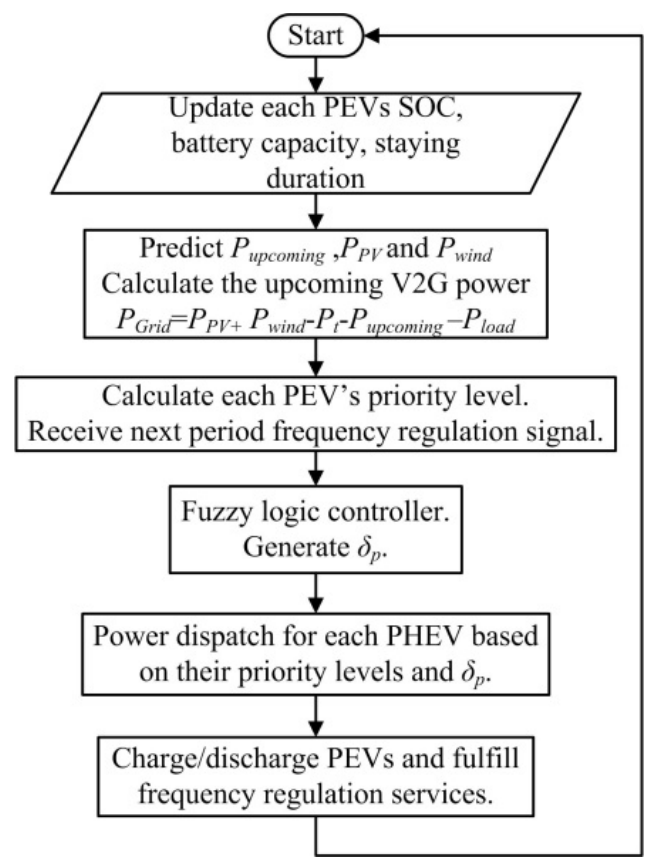

Figure 7.7 The flowchart of the developed real time fuzzy logical charging controller

\subsection{Fuzzy Logic Based Real Time Energy Management Algorithm with Consideration of Grid Frequency Regulation Services}

To reduce the impact of PEVs charging on the utility grid, PEVs with different SOCs and power requirements should be charged with different charging rates. The five charging priorities are shown in Table 7.3. Throughout the day, PEVs' priorities are varying based their SOCs and next departure times. In doing so, the PEVs will not be

TABLE 7.3 CHARgING RATES FOR DIFFERENT CHARGING LEVELS

\begin{tabular}{cccc}
\hline \hline Priority level & Power requirement & Maximum charging rate & Minimum charging rate \\
\hline Level 1 & $p \geq 15 \mathrm{~kW}$ & $12 \mathrm{~kW}$ & $12 \mathrm{~kW}$ \\
Level 2 & $10 \mathrm{~kW} \leq p<15 \mathrm{~kW}$ & $12 \mathrm{~kW}$ & $6 \mathrm{~kW}$ \\
Level 3 & $5 \mathrm{~kW} \leq p<10 \mathrm{~kW}$ & $8 \mathrm{~kW}$ & $0 \mathrm{~kW}$ \\
Level 4 & $2 \mathrm{~kW} \leq p<5 \mathrm{~kW}$ & $5 \mathrm{~kW}$ & $-5 \mathrm{~kW}$ \\
Level 5 & $p<2 \mathrm{~kW}$ & $2 \mathrm{~kW}$ & $-8 \mathrm{~kW}$ \\
\hline \hline
\end{tabular}


charged at the same rate, which will effectively limit their charging impact. Additionally, since the PEVs in lower priority levels can be discharged, they can inject power to the utility grid when the system frequency is below $60 \mathrm{~Hz}$. All PEVs can absorb power from the grid when the system frequency is above $60 \mathrm{~Hz}$. The flowchart is shown in figure 7.7.

\subsubsection{Fuzzy logic controller design}

Since the charging rate of PEVs has no direct relationship with the renewable energy farm output power and the system's load and the frequency regulation signal, a real time Mamdani-type Fuzzy Logic Controller (FLC) was designed to connect them.

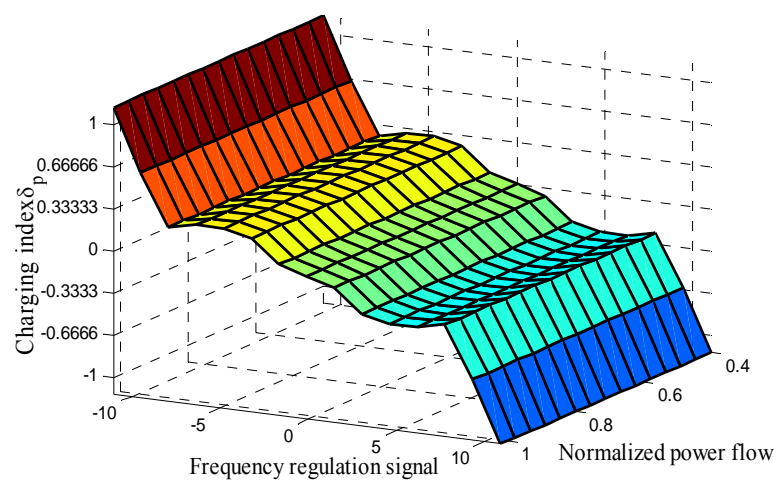

(a)



(c)



(b)



(d)

Figure 7.8 (a) Surface of the fuzzy logic controller's rules; (b) Power flow membership function; (c) frequency regulation membership function; (d) Power flow control index membership function.

The frequency regulation signal and normalized grid load flow are used as the inputs to adjust the charging rate of the PEVs. The output of the FLC is the charging 
index $\delta_{P}$, which will determine the charging rates of PEVs at different priority levels using equation (7.8). The grid power flow Pgird is described as "very low", "low", "normal", "high", and "very high". Similarly, the grid frequency regulation signal is described as "negative big", "negative", "zero", "positive", and "positive big". The method implemented for defuzzification is centroid based. Within the model, minimum and maximum are used for "AND" and "OR" operators, respectively. The parameter $\delta_{P}$ is described as "negative big", "negative", "zero", "positive", and "positive big". The output $\delta_{P}$ is in the range of $[-1,1]$. The surface of the fuzzy controller's rules and the membership functions are shown in figure 7.8 (a), (b), (c) and (d).

$$
P_{i}= \begin{cases}12 & , \text { for level } 1 \\ 9+3 \times \delta_{P} & , \text { for level } 2 \\ 4+4 \times \delta_{P} & , \text { for level } 3 \\ 0+5 \times \delta_{P} & , \text { for level } 4 \\ -3+5 \times \delta_{P} & , \text { for level } 5\end{cases}
$$

\subsubsection{Maximize the utilization of renewable energy sources}

As shown in figure 7.7, the grid power flow $P_{\text {grid }}$ is calculated based on equation (7.9). Pgrid is calculated by the central aggregator based on the current states of the components in the network. It shows the power balance situations in the PEV network. Therefore, if Pgrid is a positive number, then it means the renewable energy farm is generating more power than the total load requirement, therefore charging rates can be increased. If Pgrid is a negative number, then the PEV network may need to reduce the charging rate. However, the charging rate is not only dependent on Pgrid, the utility grid power balance situation should also take into consideration.

$$
P_{\text {Grid }}=P_{P V}+P_{\text {wind }}-\sum P_{i}-\hat{P}_{\text {upcoming } g}-P_{\text {load }}
$$


Where:

$P_{P V}$ is the forecasted next period solar farm output power. This variable is from the Neural Network forecasting results.

$P_{\text {wind }}$ is the forecasted next period wind farm output power. This variable is from the Neural Network forecasting results.

$P_{i}$ is the charging power requirement for the $\mathrm{i}^{\text {th }} \mathrm{PEV}$ that has already been connected to the charging network.

$\hat{P}_{\text {upcoming }}$ is the forecasted power requirement for the PEVs that will be connected to the charging network for the coming period. This variable can be calculated by using equation (7.4).

$P_{\text {load }}$ is a certain percentage of the forecasted next local load. This variable is from the Neural Network forecasting results.

\subsubsection{Regulating the grid frequency by using the PEV network}

The utility grid active power balance situation is reflected by the system frequency. If the frequency is higher than the rated value, some active power should be absorbed by the PEV network to decrease the grid frequency. If the grid frequency is lower than the rated value, the PEV network needs to inject active power to the utility grid to feed the extra load. Therefore, the grid frequency regulation signal is also used as one of the inputs for the fuzzy logic control proposed in section 7.6.1.

\subsection{Overall System Economic Analysis}

The grid frequency regulation service may degrade the battery lifetime and capacity, so in order to analyze the practical feasibility of the proposed model, an 
economic evaluation plan involving frequency regulation revenue should be established. Without benefits, the owners of PEVs would be unlikely to let their vehicles join the network and perform the V2G services. For the PEV network system with the renewable energy farm, the daily revenue can be calculated by equation (7.10).

$$
R_{\text {total }}=\int_{t=0}^{t=24} P_{r}(t) \times p_{r, \text { price }} d t+\left(E_{\text {solar }}+E_{\text {wind }}\right) \times p_{g, \text { price }}
$$

where

$P_{r, p r i c e}$ is the unit frequency regulation power rate $(\$ / \mathrm{kWh})$,

$P_{g, p r i c e}$ is the unit electricity power rate $(\$ / \mathrm{kWh})$.

The daily cost for the PEV network system involves the cost of energy purchased for the driving needs of the vehicles, the cost of battery replacement and maintenance, and the capital cost. The total daily costs of the PEV network can be calculated by equation (7.11).

$$
\begin{aligned}
& C_{\text {total }}=C_{R F} \sum_{i=1}^{50,000}\left(\frac{C_{b} \times B_{c, i}+p_{\text {install }, i}}{L_{c} \times B_{c, i} \times D o D} \times M_{d, i} \times E_{m, i}\right) \\
& +\sum_{i=1}^{50,000}\left(M_{d, i} \times E_{m, i} \times p_{g, p r i c e}\right) \\
& C_{R F}=\frac{R_{\text {int erest }}}{365 \times\left(1-\left(1-R_{i}\right)\right)^{-n}}
\end{aligned}
$$

where

$$
\begin{array}{ll}
C_{b} & \text { is the unit cost of battery }(\$ / \mathrm{kWh}), \\
C_{R F} & \text { is the daily capital cost }(\$), \\
D o D & \text { is the maximum depth of discharge (\%), } \\
E_{m, i} & \text { is the } \left.i^{\text {th }} \mathrm{PEV} \text { efficiency (miles } / \mathrm{kWh}\right),
\end{array}
$$




$$
\begin{array}{ll}
L_{c} & \text { is the lifetime in cycles, } \\
M_{d, i} & \text { is daily driven mileage for the } i^{\text {th }} \text { PEV (mile), } \\
n & \text { is the average battery lifetime years, } \\
P_{\text {install }} & \text { is the battery installation fee }(\$), \\
R_{\text {interest }} & \text { is the annual interest rate }(\%) .
\end{array}
$$

Since the maximum frequency regulation requirement is no more than $13 \mathrm{MW}$ during the day and the PEV network can easily support this amount of power, there will be no penalty issues. Therefore, the PEV smart charging network's daily income can be calculated simply by using $R_{\text {total }}$ minus $C_{\text {total }}$. This is just a simplified economic analysis model with the assumption that unit electricity rate is constant during the whole day. However, in reality the electricity rate is varying with time, higher during the load peak period and lower during the load valley time. The smart charging algorithm can limit the charging impact of PEVs to the grid. This results in PEVs having higher power charging ratings during off-peak periods and lower power charging ratings during peak periods with the ability to inject power back into the grid. Therefore, the real cost should be lower than the result calculated by equation (10). Additionally, this chapter doesn't take other types of $\mathrm{V} 2 \mathrm{G}$ auxiliary services such as spinning reserve, voltage regulation, and reactive power compensation into consideration. Therefore, the real revenue should be higher than the estimated results obtained from the proposed model. The complex economic analysis will be conducted in future work. 


\subsection{System Simulation and Analysis}

In order to validate whether the proposed PEV network smart charging algorithm can earn revenue by limiting the impact of charging PEVs to the grid and regulating the grid's system frequency, the proposed 50,000 PEVs network 24-hour charging behavior was simulated in two cases: charging with and without smart power management. For general study, the solar irradiance and wind speed data used are the average daily values in August, 2010, and the local load data used is the average daily value in August, 1987, South Florida area. The charging index $\delta_{p}$ was generated by the central aggregator every 6 minutes to adjust the charging rates for the PEVs in the network. For protection purposes, the SOCs of the batteries should stay above $20 \%$. Also, the SOCs of the PEVs should be above $85 \%$ at the next departure time, which will make sure the V2G service will not influence the drivers' driving behaviors.



Figure 7.9 Charging of PEV network without smart charging algorithm

The simulation result of the PEV network charging process during a single day without smart charging management is shown in figure 7.9. This case simulates the 24hour charging process without cooperation between the PEV network and the utility grid. 
Whenever a PEV is connected to the grid, it will be charged with a constant charging rate of $12 \mathrm{~kW}$, and won't stop charging until the SOC of its battery reaches $85 \%$. As the result shows, the charging of the PEV network greatly impacted the utility grid by increasing the load peak from $1180 \mathrm{MW}$ to $1276 \mathrm{MW}$. The charging of PEVs brought two load peaks which happened around 9 AM and 18 PM respectively. This is due to PEVs' charging after periods of travelling from home to the work place in the morning and from the work place back home in the afternoon. Also, we should notice that there are no charging behaviors during the night hours (22 PM to $6 \mathrm{AM}$ ), because the PEVs were already charged and the SOCs of the batteries were already above the limit of $85 \%$. Therefore, the PEV network couldn't help the utility grid fill the valley. Also, the charging of the PEVs could be more expensive since in reality the electricity price is usually high during the load peak hours and low during the load valley hours.

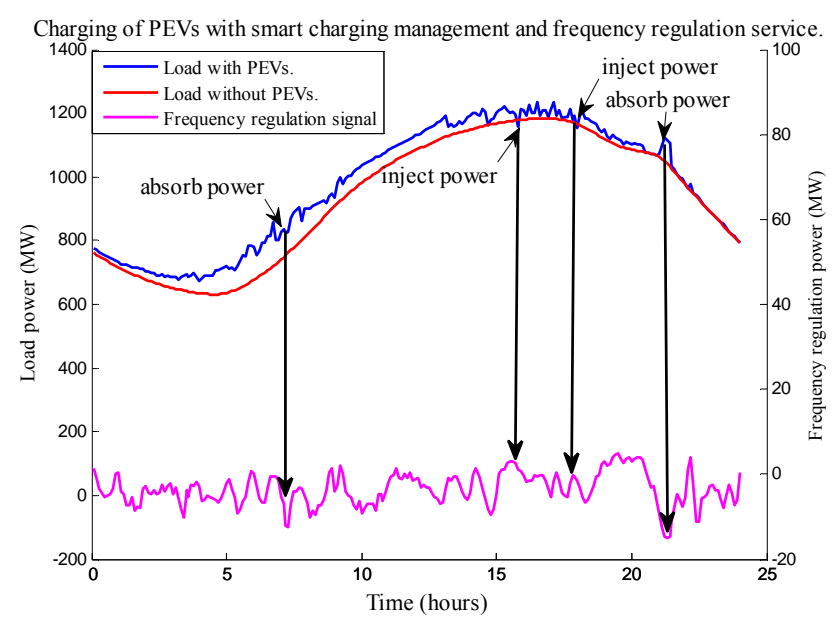

Figure 7.10 Charging of PEV network with smart charging algorithm

The simulation result of the PEV network charging process during a single day with smart charging management is shown in figure 7.10. In this case, the proposed real time smart charging management algorithm was applied to the PEV charging process. 
The charging of PEVs was distributed throughout the day, so instead of being charged randomly, the PEVs are smoothly charged in a well-organized manner. Additionally, most of the charging processes occurred during the load off peak period. The grid load peak with PEVs is only 1215 MW. Figure 7.10 also shows the frequency regulation capability of the proposed system. The positive regulation power means the power generated in the utility grid is less than the system load, which will cause the system frequency to decrease. Therefore, extra power is needed to inject to the utility grid from the PEV network to regulate the system frequency back to $60 \mathrm{~Hz}$. On the contrary, if the frequency regulation power is negative, the PEV network is used to absorb the extra power in the utility grid to decrease the frequency rate back to the normal value. The simulation results show that the proposed system can properly follow the frequency regulation signal and help the grid adjust the system frequency.

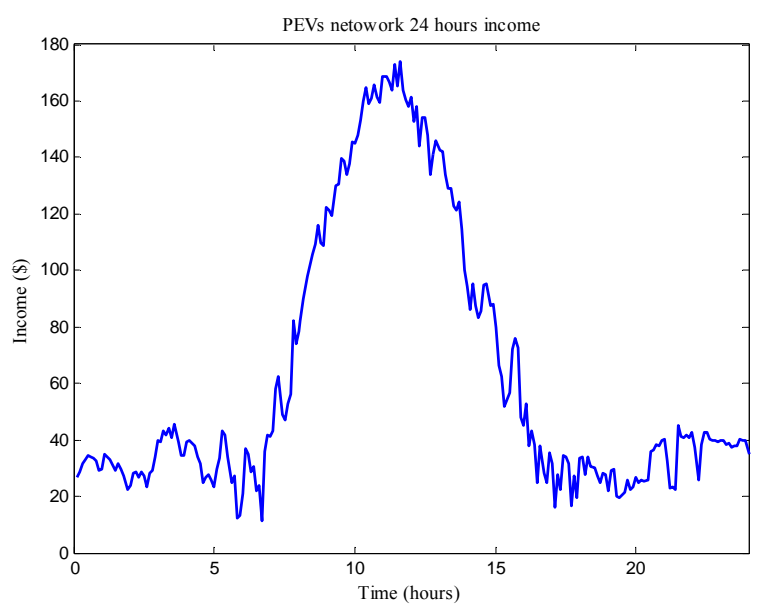

Figure 7.11 24-hour PEV network income

For the economic analysis of the proposed model, simulations for a single day and for an entire year are done based on equations (7.10) to (7.12) in MATLAB. In the simulation, for simplification, the unit electricity rate and unit frequency regulation rate 
are set constant as $0.04 \$ / \mathrm{kWh}$ and $0.06 \$ / \mathrm{kWh}$ during the whole day. The battery unit cost is set as $689 \$ / \mathrm{kWh}$, maximum depth of discharge is set at $80 \%$, average battery lifetime is 10 years, and annual interest rate is $5 \%$. Figure 7.11 shows the simulation result of the 24-hour PEV network income based on case two. Because of the V2G service and the energy generated by the renewable farm, the income is always above zero, which means the proposed system will keep earning money. The income for this single day is $\$ 152,450$. The peak of the income appears around noon since in this period the PV panels in the renewable energy system generate more power. Another reason is that during noon, most of the PEVs' batteries have already been charged over a certain level. They stop charging with high charging rates and begin involving more in the V2G services.

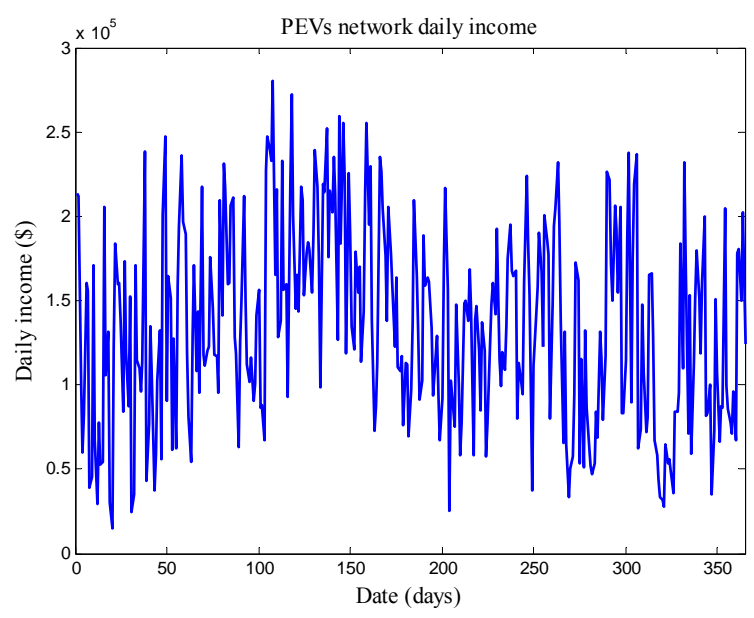

Figure 7.12 One year PEV network daily incomes

To cover all the different cases in practical situations, an economic analysis of the simulation results for an entire year of the proposed model is shown in figure 7.12. In this simulation, the one year data of solar irradiance, wind speed, local load power flow, and frequency regulation was used to estimate the annual income. The simulation results show that the proposed PEV network and the scheduled renewable farm can earn benefits 
every day throughout the year. Because of variances in the solar irradiance and wind speed, the daily incomes are varying from below $\$ 50,000$ to above $\$ 250,000$. The annual income is $\$ 49.463$ million. The annual income is the net margin for the PEV network and its customers. The battery replacement fee and the degradation cost have already been covered. The annual income can be used to compensate for the establishment cost of the renewable energy farm and to pay customers to encourage them to join their PEVs into the PEV network and participate in the V2G services. By doing this, more PEVs will join the smart charging PEV network in the future. This will boost the development and utilization of renewable energy while at the same time increasing the stability of the power system.

\subsection{Conclusion}

In this chapter, the economic analysis of a real-time smart charging algorithm for 50,000 PEVs in a network with the consideration of V2G frequency regulation service was studied. A real-time energy management algorithm for the PEV network with renewable farm based on charging priority levels was proposed. The algorithm aims at minimizing the PEVs' charging impact to the utility grid while at the same time regulating the frequency of the grid. The charging rates of the PEVs during their parking period are varying according to their state of charge. A fuzzy logic agent was used as a component within the developed algorithm. An economic model was built to estimate the daily and annual income of the proposed system with the consideration of battery degradation. The performance of the developed algorithm and the economic analysis of the whole model were tested by simulation. The economic analysis of the proposed 
system shows the capability of bringing considerable benefits to both the network and the owners of the PEVs. 


\section{Bidirectional Energy Transfer and Power Electronics Devices Modeling, Simulation and Hardware Implementation}

\subsection{Introduction}

Hybrid AC-DC power systems are growing in popularity due to the increase in micro grids implementing renewable power conversion systems connected to low voltage $\mathrm{AC}$ distribution systems. This growth has also been attributed to the environmental issues caused by conventional fossil fueled power plants [151]-[153]. Furthermore, DC grids are resurging due to the development of new semiconductor techniques and sustainable DC power sources such as solar energy. There has also been an increase in DC loads, such as plug-in electric vehicles (PEVs) and light emitting diodes (LEDs), connected to the grid to save energy and decrease greenhouse gas emissions. The PEVs can be viewed as energy storage devices when they are parked in a garage, allowing them to increase the stability and efficiency of the micro grid they are connected to. One of the major technical challenges in micro grids is the interconnection of a pulse load which can cause voltage collapse, oscillation of the angular velocity in the generators, and degradation of the overall system performance.

Researchers have proposed several ideas and models relating to renewable energy sources and storage, ranging from their scheduling and PEV charging optimizations to the feasibility of PEV vehicle-to-grid (V2G) services [34], [59], [145]. However, these models only propose the idea without thorough analysis of the of the energy conversion 
between the AC and DC sides. Researchers have also proposed several ideas and models of AC-DC micro grids [24], [114], [155], but their systems operate without the influence of critical loads. System stability and coordination control of power electronics devices during grid-connected and islanding modes with the influence of critical loads is still an open issue.

At the same time, various utility grids and some hybrid AC-DC power systems are increasing the penetration of renewable energy resources [156]-[157]. This growth in renewable sources increased the challenge these systems meet due to the intermittent nature of wind and solar power. This can quickly add up to system-wide instability that can force generators to ramp up and down wildly, push grid protection gear into states it's not meant to handle, or force the wind and solar generator to shut off altogether [158]. Hybrid power systems face far more challenges when operating in islanding mode than in grid connected mode. During islanding mode, the AC side can no longer be viewed as an infinite bus, which results in load variations adversely affecting the frequency and voltage of the system. If the system has a high penetration of renewable power, the situation can be even worse. At any time, power flow should be balanced between the AC and DC sides to maintain stability on both sides of the grid. Also, both reactive and active power in the $\mathrm{AC}$ side of the system should be balanced to keep the frequency and voltage stable.

This chapter proposes distributed power control for a hybrid AC-DC power system with a renewable energy source, energy storage devices, and pulse loads. The proposed hybrid AC-DC power system consists of both AC and DC subsystems with a synchronous generator and a PV farm as power supplies, respectively. A fully controlled 
bidirectional AC-DC interlinking converter is used to link the AC subsystem with the DC subsystem. A DC-DC boost converter with maximum power point tracking (MPPT) function is implemented to maximize the energy generation from the PV farm. A Bidirectional DC-DC converter is applied to connect the lithium-ion battery bank in the DC subsystem to act as an energy storage device that serves to increase the system stability. The detailed power control strategies of different power electronics devices for the hybrid micro grid operated in grid-connected mode and islanding mode are proposed and analyzed. Simulation and hardware experiments are done with the mitigation of pulse loads. The results verify that the proposed topology is coordinated for power management in both AC and DC subsystems under critical loads with significant improvement for the system in terms of voltage and frequency stability.

This chapter is organized as follows; hybrid AC-DC power system operation in grid-connected and islanding modes together with their islanding detection and frequency regulation methods are presented in Section 8.2. Section 8.3 presents the detailed description of the topology, control, and hardware design of a bidirectional DC-DC converter and its real-time experimental verification. The bidirectional AC-DC inverter topology, vector decoupling control, frequency and voltage control, hardware description and real-time experimental verification is given in Section 8.4. Section 8.5 shows the simulation and hardware experimental verification of the proposed hybrid power system control strategy with the mitigation of critical loads. Finally, a conclusion is given in Section 8.6. 


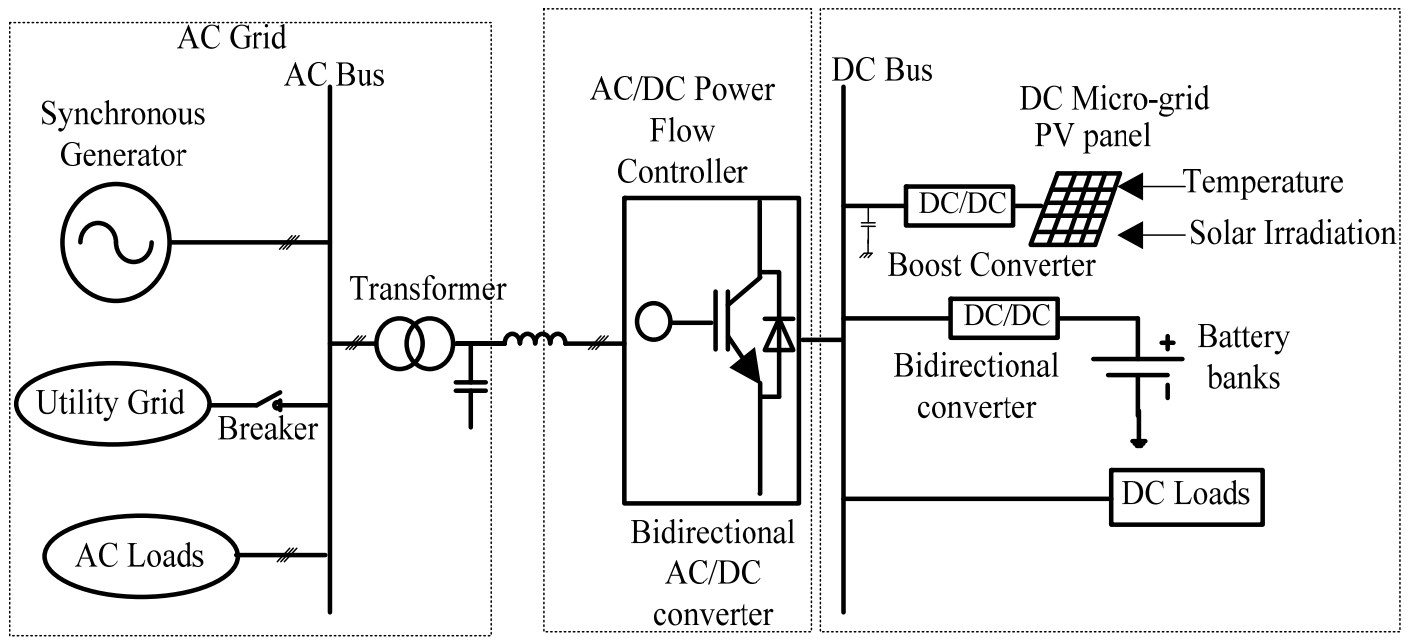

Figure 8.1 Hybrid AC-DC micro grid configurations.

\subsection{Hybrid AC-DC Power System Operation in Grid-connected Mode and Islanding Mode}

Hybrid AC-DC micro grids comprise low voltage distribution systems with distributed energy resources, such as photovoltaic power systems and wind turbines, together with storage devices. These systems are interconnected to the medium voltage distribution network, but they can be also operated isolated from the main grid.

Figure 8.1 shows the hybrid micro grid configuration where a synchronous generator, a PV farm, and loads are connected to its corresponding AC and DC sides. The $\mathrm{AC}$ and $\mathrm{DC}$ sides are linked through a bidirectional three phase $\mathrm{AC}-\mathrm{DC}$ converter and a transformer. The system has constant loads and pulse loads on both the AC and DC sides. A PV farm is connected to the DC bus as the DC energy source through a DC-DC boost converter with MPPT functionality. Local synchronous generators are connected to the AC side. Battery banks are connected to the DC bus through bidirectional DC-DC boost converters. Pulse loads are connected to AC and DC sides respectively. 


\subsubsection{Hybrid AC-DC power system operation in grid-connected mode}

In grid-connected mode, there is a connection between the hybrid power system and the utility grid. Because of this, the AC bus of the hybrid power system can be viewed as an infinite bus with constant frequency and voltage amplitude. Therefore, no regulation is needed on the AC side.

The hybrid power system only needs to control the DC bus voltage at the rating level. The bidirectional AC-DC inverter takes charge of regulating the DC bus voltage, and the PV boost converter is operated in MPPT mode to maximize the renewable energy utilization. Therefore, in grid-connected mode, the energy storage system in the hybrid power system is not essential since the DC bus voltage is regulated by the bidirectional AC-DC inverter.

\subsubsection{Hybrid AC-DC power system operation in islanding mode}

Islanding operation means that when faults happen in the grid, the micro-grid is isolated from the remainder of the utility system and distributed generators supply power to the load. Islanding can be harmful: firstly, due to the islanding area being out of the grid's control, the voltage and frequency may be unstable and it will threaten safety operation of electrical equipment; secondly, the main grid would be impacted when the micro-grid is reconnected with the main grid.

In islanding mode, the AC bus of the hybrid power system can't be viewed as an infinite bus anymore. Therefore, both the frequency and the voltage amplitude need to be regulated. In this mode, if the system has a slack generator, it will regulate the system frequency. Otherwise, the bidirectional AC-DC inverter takes charge of regulating the frequency and voltage amplitude by controlling the active and reactive power, 
respectively. On the DC side, the DC bus voltage also needs to be regulated. Because of this, the bidirectional DC-DC converter will control the charging/discharging rate of the energy storage system to make the DC bus voltage stable. Therefore, the energy storage system plays an important role when the hybrid power system operates in islanding mode.

\subsubsection{Hybrid AC-DC power system islanding detection and frequency regulation}

The most important feature of hybrid AC-DC power systems is that they have the ability to be disconnected from or synchronize with the utility grid. Generally, a transition-to-island mode can be a result of scheduled or unscheduled events. Scheduled transitions are intentional events for which the time and duration of the planned island are agreed upon by all parties involved. Unscheduled transitions are inadvertent events that are typically initiated by the loss of an area of the utility grid or equipment failure, and the hybrid AC-DC power system needs to be automatically sectionalized from the local utility grid by islanding protective relay.

For example in figure 8.1 , if a fault happens in the utility grid outside the hybrid AC-DC power system near to the grid connection circuit breaker, the frequency and voltage of the connection point may dramatically drop in a short time. To protect the hybrid power system, the breaker is supposed to be disconnected to isolate the hybrid power system from the main grid.

In the hybrid AC-DC power system, assume the active and reactive power outputs form the distributed generators are $P$ and $Q$, and the power loads in the hybrid power system are represented as $R, L$ and $C$. Then in islanding operation, 


$$
\begin{aligned}
& P=\frac{V_{A C}^{2}}{R} \\
& Q=V_{A C}^{2}\left(\frac{1}{\omega L-\omega C}\right)
\end{aligned}
$$

Therefore

$$
\begin{gathered}
V_{A C}^{2}=P R \\
\omega=\omega_{0}\left(\sqrt{\frac{1}{4 \lambda^{2}}\left(\frac{Q}{P}\right)^{2}}+1-\frac{Q}{2 \lambda P}\right)
\end{gathered}
$$

Where, $\omega_{0}=1 / \sqrt{L C}$ is the syntonic angular frequency and $\lambda=R \sqrt{C / L}$ is the power factor of the loads. Equations (8.3) and (8.4) show that the voltage and frequency may deviate from the rated values when the hybrid power system transfers into islanding mode because there is generally an imbalance between the generation and the load when the system is operated in islanding mode. Furthermore, in the hybrid power system AC subsystem, the change of voltage is related to active power imbalance while the frequency shift is mainly based on reactive power imbalance. Consequently, over/under voltage and over/under frequency can be used as the signal to detect islanding.

Another scenario is frequency regulation for hybrid AC-DC power systems in islanding mode. During islanding operation, if there is an $\mathrm{AC}$ generator acting as the slack generator, then it will take charge of the whole system frequency regulation. No more than one slack generator is allowed in the hybrid AC-DC power system to avoid fighting with each other. In this situation, the bidirectional AC-DC inverter can only be operated in current control mode, which will not regulate the AC side frequency and voltage amplitude. However, under certain circumstances such as when a fault happens, 
the slack generator may disconnect from the rest of the hybrid power system, which will make the rest of the system frequency dramatically oscillate. Therefore, the bidirectional AC-DC inverter needs to detect the frequency variation and convert from current controlled converter to voltage controlled converter to immediately take charge of the system frequency and voltage amplitude regulation. However, for hybrid power systems operated in islanding mode, some pulse loads can also cause huge system frequency oscillation even if the slack generator is still connected. In this case, the bidirectional ACDC inverter should stay in current control mode, which will prevent it from fighting with the slack generator.

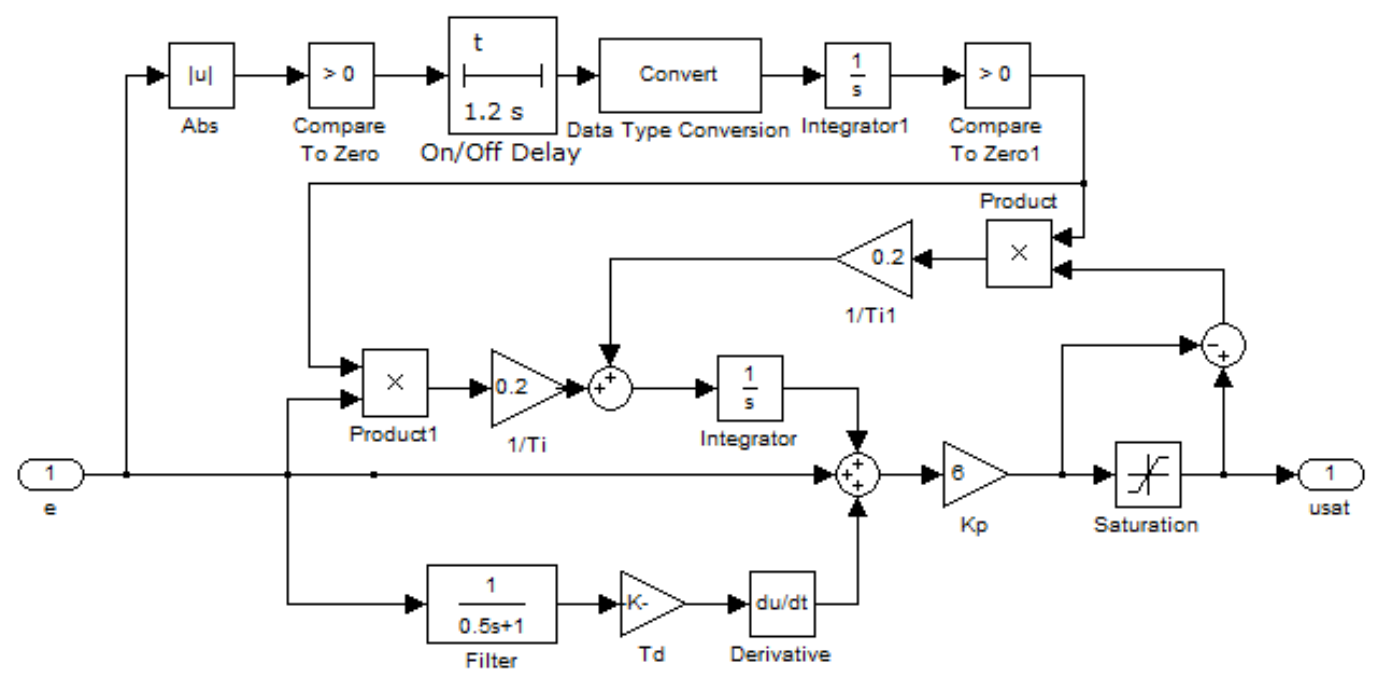

Figure 8.2 Bidirectional AC-DC inverter frequency regulation enable program

Therefore, for hybrid AC-DC power systems, an effective control scheme should be designed to control the system voltage and frequency during islanding operation with pulse load disturbances. In this chapter, a smart islanding detection and modes switching algorithm is designed for control of the bidirectional AC-DC inverter. The detailed experimental results are shown in Chapter 10. The proposed system has two kinds of 
pulse loads with $600 \mathrm{~W}$ and $300 \mathrm{~W}$ power consumptions. The periods of these two pulse loads are 1 second. Therefore, the islanding detection algorithm is designed to be enabled if the frequency variations exceed the limit for longer than 1.2 seconds. The control program of the frequency regulation is shown in figure 8.2.

\subsection{Bidirectional DC-DC Converter Control and DC Bus Voltage Regulation}

\subsubsection{Bidirectional DC-DC converter topology}

The topology of the bidirectional DC-DC converter is shown in figure 8.3. There are two switches, D1 and D2. The battery is connected to the left side of the bidirectional DC-DC converter, which is the low voltage side. The DC bus is connected to the high voltage side of the converter.

Switches D1 and D2 can't be operated at the same time. When D1 is operated, the bidirectional DC-DC converter is working in buck mode. Power flows from the DC bus to the battery, which is the charging process. When D2 is operated, the bidirectional DCDC converter is working in boost mode, power flows from the battery to the DC bus, and the battery is discharged.

\subsubsection{Bidirectional DC-DC converter current and voltage control}

In grid-connected mode, the bidirectional DC-DC converters only regulate the

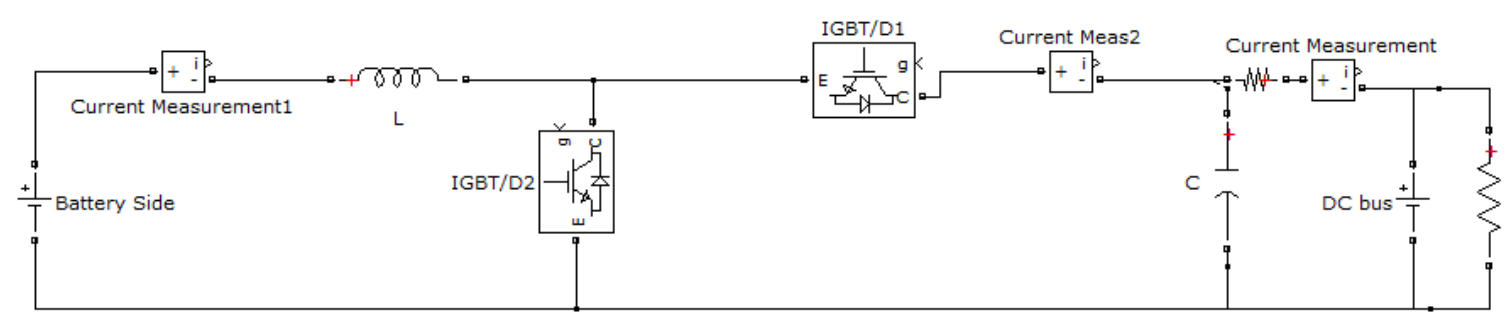

Figure 8.3 Topology of the bidirectional DC-DC converter. 
battery banks (or PEVs) charging rates. Based on the SOCs of the battery banks and the power flow conditions on the $\mathrm{AC}$ side, the charging/discharging current references are generated to regulate the current flow in the converters. Each battery has its own bidirectional DC-DC converter, which means they can have different charging rates. By doing this, the battery banks can inject power into or absorb power from the DC bus, and even transfer energy between different battery banks if necessary. In this case, only one current control closed loop with PI controller is enough to regulate the current. The bidirectional DC-DC converter PWM signal is generated through the control block shown in figure 8.4.

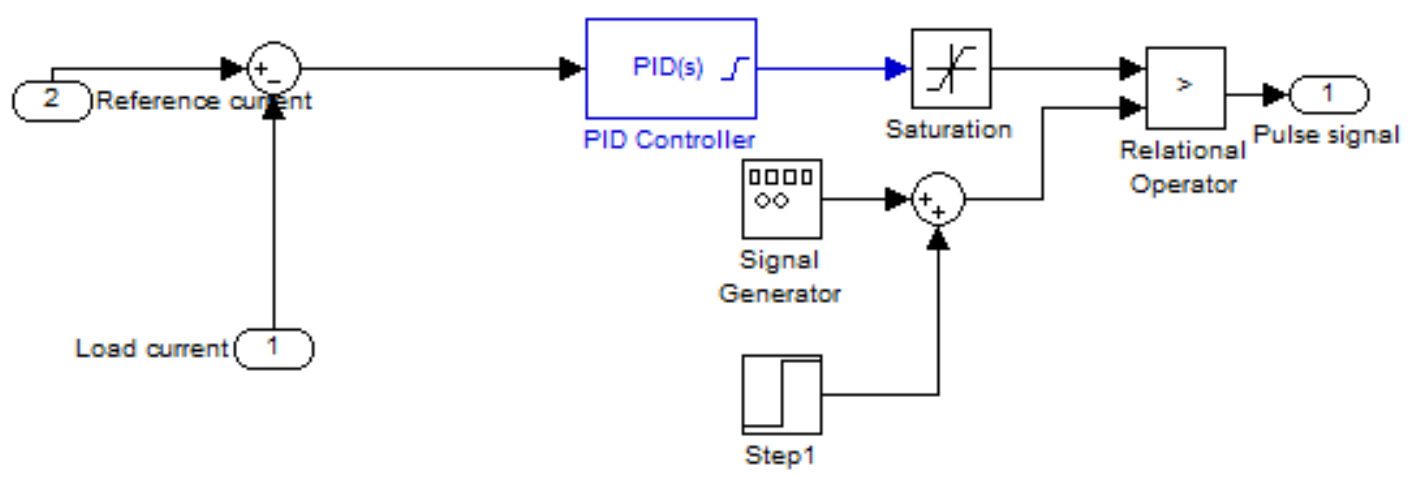

Figure 8.4 Bidirectional DC-DC converter current control program.

The error between the reference current and the measured current is sent to the PI controller which generates the duty cycle for the IGBT D1 or D2. An anti-windup PI controller controls the bidirectional DC-DC converter by means of adjusting the duty cycle, which leads to the proper PWM pulses for triggering the IGBT switch. The antiwindup controller prevents the integrator from accumulating the error, when the controller output goes to the saturation region, i.e. duty cycle greater than 1 or less than 0 . For protection proposes, the output from the PID controller is limited within the range of 
$[0.05,0.95]$. This approach helps the controller to quickly recover from the saturation region after observing a change in the sign of the error signal.

The switches chosen circuit is shown in figure 8.5. The reference signal is compared with two very small numbers such as -0.01 and 0.01 . If the reference signal is larger than 0.01 , then it works in boost mode, the Relational Operator output is 0 , and the Relational Operator 1 output is 1 . Because of this, the value after the Product block is always 0 , which is the signal for Switch D1. The signal after the Product1 block is the PWM signal from port 2, and this signal is finally sent to Switch D2 to make the converter work in boost mode. If the reference signal is smaller than -0.01 , then it works in buck mode, the Relational Operator output is 1 and the Relational Operator1 output is 0 . Because of this, the value after the Product1 block is always 0 , which is the signal for Switch D2. The signal after the Product block is the PWM signal from port 2, and this signal is finally sent to Switch D1 to make the converter work in buck mode.

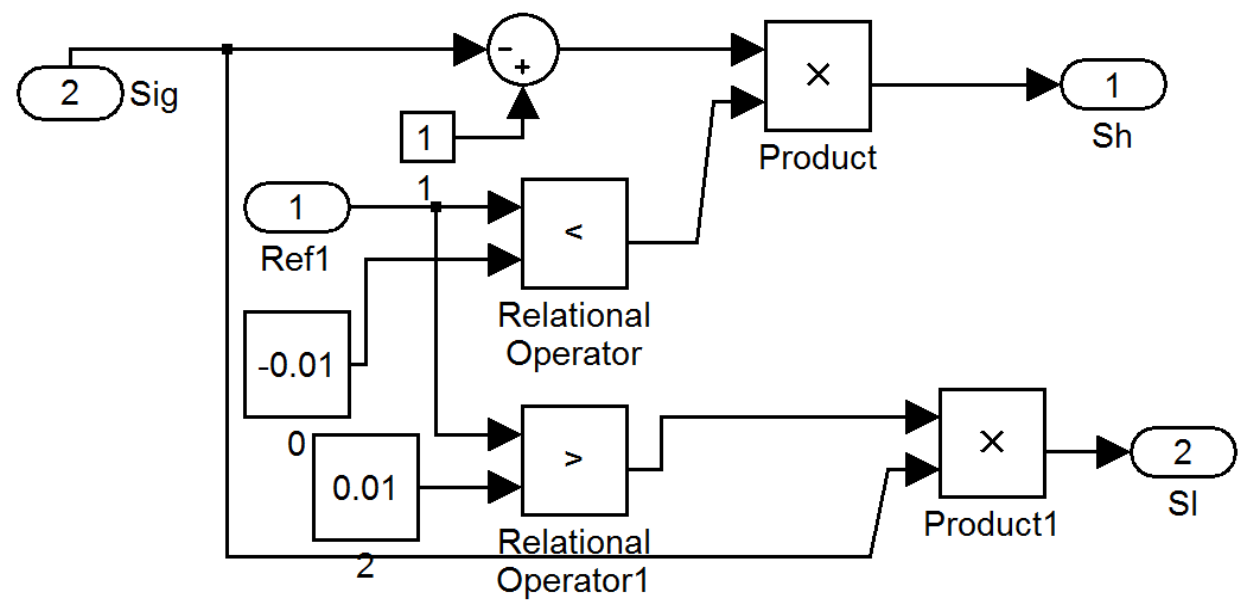

Figure 8.5 Bidirectional DC-DC converter switches chosen circuit 


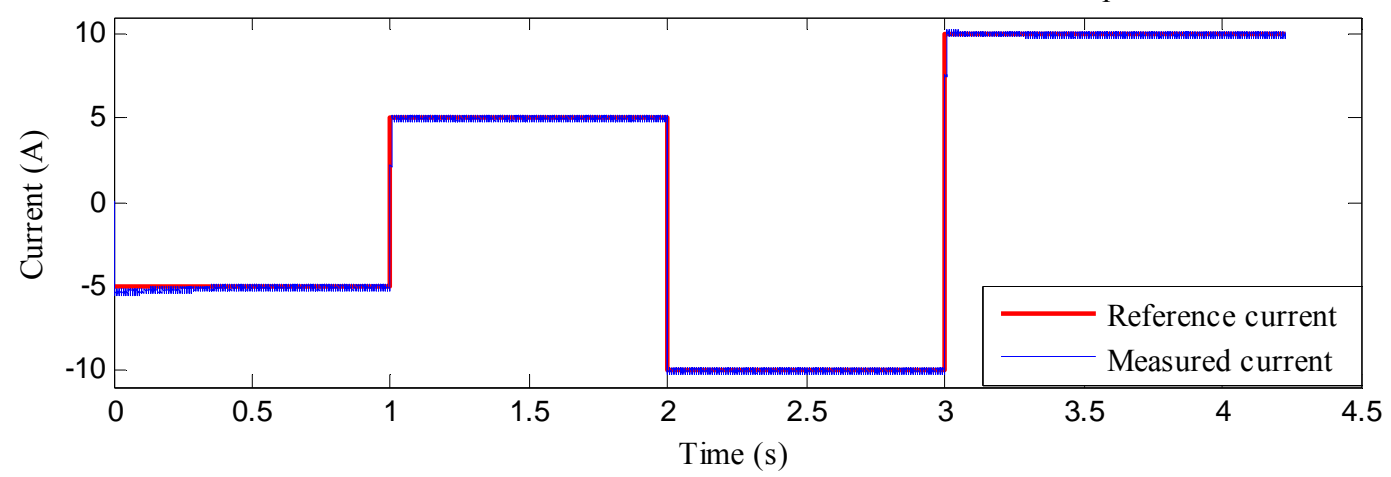

Figure 8.6 Simulation of bidirectional DC-DC converter current control response

The simulation result of the current controlled bidirectional DC-DC converter is shown in figure 8.6. The initial reference current is set as $-5 \mathrm{~A}$. From the simulation we can see the measured real current can start with $-5 \mathrm{~A}$ without delay and overshot. At time $\mathrm{t}$ $=1$ second, the reference current changed from $-5 \mathrm{~A}$ to $5 \mathrm{~A}$, and the reference current changed to $-10 \mathrm{~A}$ and $10 \mathrm{~A}$ at $\mathrm{t}=2$ seconds and 3 seconds respectively. The measured current can closely follow the reference value with almost no overshot or delay during transient response and with no ripple in steady state.

\subsubsection{Bidirectional DC-DC converter hardware}

The PCB based bidirectional DC-DC converter is shown in figure 8.7. The Table 8.1 Specifications and parameters for Bidirectional DC-DC Converter

\begin{tabular}{ccc}
\hline \hline Symbol & Quantity & Value \\
\hline$P$ & Power Rating & $2 \mathrm{~kW}$ \\
$V_{d c}$ & High voltage side voltage & $100-450 \mathrm{~V}$ \\
$V_{b}$ & Low voltage side voltage & $51.8 \mathrm{~V}-200 \mathrm{~V}$ \\
$C$ & High voltage side capacitance & $3600 \mu f$ \\
$L$ & Input Inductor & $2.2 \mathrm{mH}$ \\
$f_{s}$ & Switching frequency & $10 \mathrm{kHz}$ \\
\hline \hline
\end{tabular}


parameters of each component are shown in Table 8.1. This circuit has two HGTG30N60C3D IGBTs, corresponding to the two switches shown in figure 8.3.

A FOD 3120 Opt coupler is added to the circuit board for protection. Also, the Vinfinity voltage source is used to boost the PWM signal $15 \mathrm{~V}$ which makes it possible to drive the IGBTs by using the PWM signal generated from low voltage micro controllers whose output voltage is only $3.3 \mathrm{~V}$ or $5 \mathrm{~V}$. Protection fuses are also added to the design. The current is limited from $-10 \mathrm{~A}$ to $10 \mathrm{~A}$ to make the power flow within $2 \mathrm{~kW}$, which is enough for laboratory scale usage. The input inductor $(L)$ value was selected such that the boost converter operates in the continuous conduction mode, while its capacitor was designed such that the output ripple does not exceed 5\%.

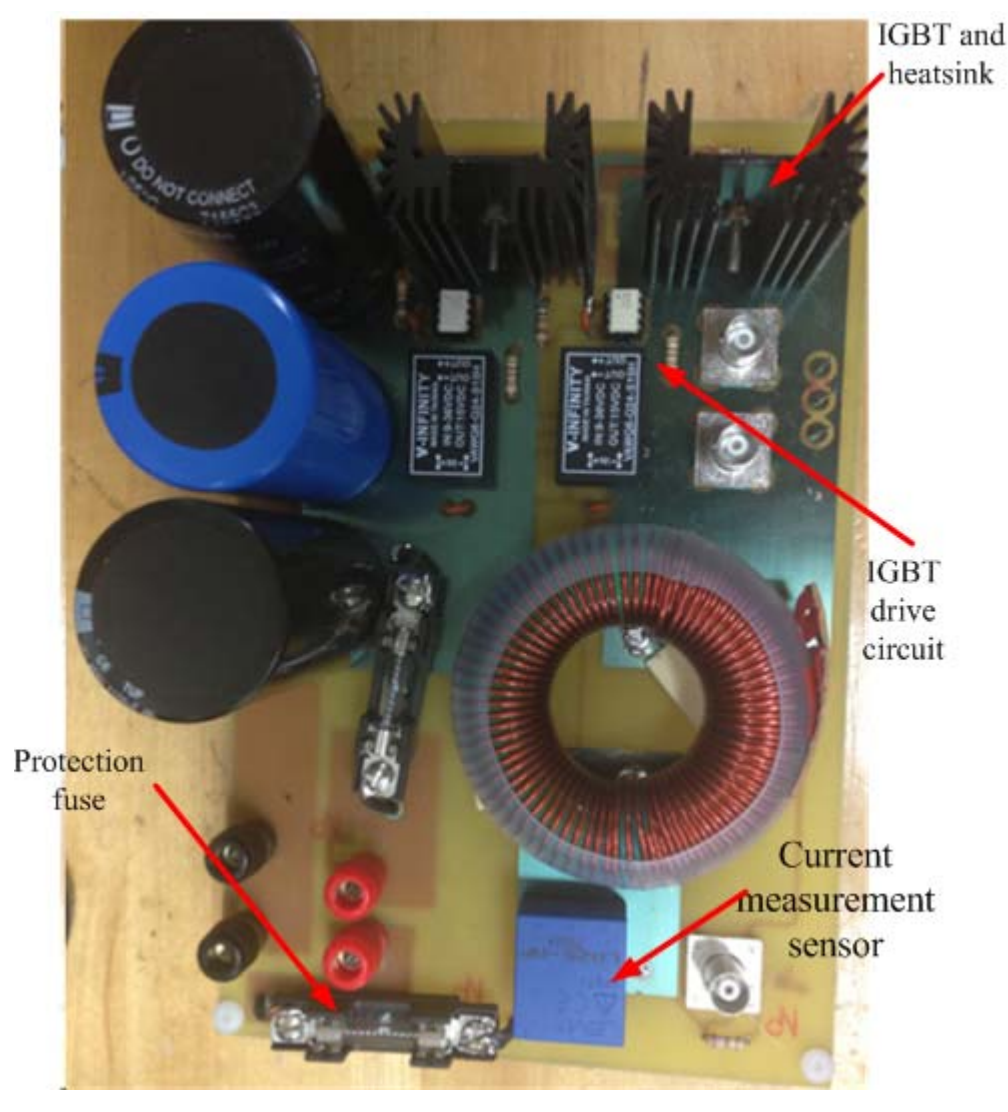

Figure 8.7 Bidirectional DC-DC converter hardware circuits. 


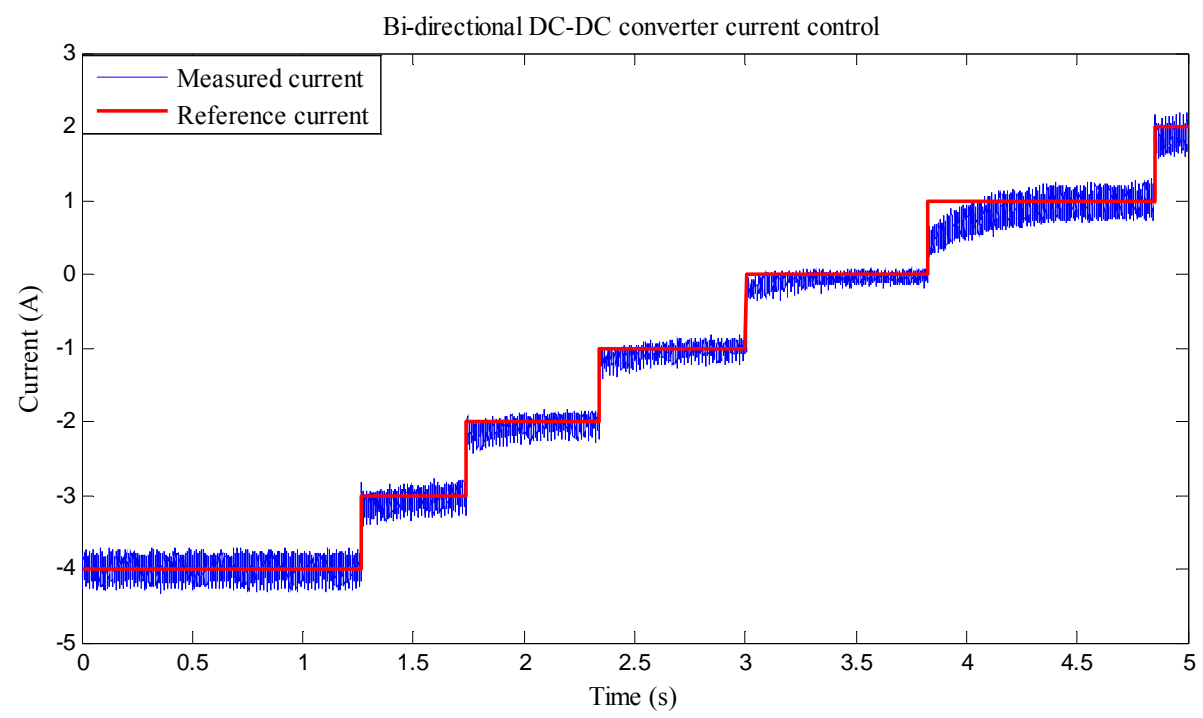

Figure 8.8 Bidirectional DC-DC converter current control experimental result.

\subsubsection{Real time hardware experimental verification}

A hardware experiment of controlling the bidirectional current flow was done to verify the performance of the bidirectional DC-DC converter. The low voltage side is connected with a lithium-ion battery bank with terminal voltage of $51.8 \mathrm{~V}$. The high voltage side of the converter is connected to the DC bus with a voltage of $120 \mathrm{~V}$. The hardware experimental result of the bidirectional DC-DC converter current control is shown in figure 8.8 . The battery was discharged with a $-4 \mathrm{~A}$ reference charging signal at the initial time. The charging signal was changed to $-3 \mathrm{~A},-2 \mathrm{~A},-1 \mathrm{~A}, 0 \mathrm{~A}, 1 \mathrm{~A}$, and $2 \mathrm{~A}$, at $\mathrm{t}$ $=1.3,1.75,2.45,3.05,3.9$, and 4.85 seconds respectively. From this hardware experiment it can be seen that the current flow through the bidirectional DC-DC converter can closely follow the reference charging signal with a fast response and no overshot. The current ripple generated by the switching of the IGBTs is at an acceptable range in the steady state. 
This bidirectional DC-DC converter can be used as the charger for lithium-ion battery banks. It can also be viewed as a PEV charger that has the ability of injecting power from the battery to fulfill the V2G functions. Furthermore, the designed bidirectional DC-DC converter can be controlled by microcontrollers with wireless communicators. Then the power flow through this converter can be remotely controlled, which can be used for managing the charging process of further large PEV networks as proposed in Chapter 7. The detailed bidirectional DC-DC converter remote control is given in Chapter 9.

\subsection{Bidirectional AC-DC inverter control and AC Bus Frequency and Voltage Regulation}

The bidirectional AC-DC inverter plays an important role in hybrid AC-DC power systems in both grid-connected and islanding modes. An important feature of hybrid power systems is the ability to transfer power between the AC and DC sides. In grid connected mode, power flow from the AC to DC side is very important to cover any deficiency in the demand on the DC grid due to normal or pulsed loading. Moreover, power flow from the $\mathrm{DC}$ to $\mathrm{AC}$ grid is needed when there is extra power from renewable energy sources on the DC bus. In islanding mode, power flow between the AC and DC side of the hybrid power system is used to cover the active and reactive power deficiency in the demand, which may cause the AC subsystem frequency or voltage amplitude to drop. Also, the DC side can absorb active or reactive power when there is extra power from the generator on the AC bus or sudden heavy load disconnection. 


\subsubsection{Bidirectional AC-DC inverter topology}

The topology of the three phase full bridge bidirectional AC-DC inverter is shown in figure 8.9. The inductor $L$ that works as a line filter is mounted between the AC subsystem and the DC subsystem. The capacitor $C$ is used to depress the DC bus voltage variations.



Figure 8.9 Bidirectional AC-DC inverter topology

The phase potentials of the AC subsystem are denoted $u_{a}(t), u_{b}(t)$ and $u_{c}(t) . \omega$ is the AC subsystem voltage angular frequency. The current flows from the DC side to the inverter are denoted as $i_{a}(t), i_{b}(t)$ and $i_{c}(t)$, while the DC link voltage and current are denoted as $v_{d c}(t)$ and $i_{d c}(t)$, respectively.

\subsubsection{Bidirectional AC-DC inverter vector decoupling technique}

To design and control a bidirectional AC-DC inverter, mathematical models are important tools for predicting dynamic performance and stability limits. The simplified model of the bidirectional AC-DC inverter is shown in figure 8.10.

If the $\mathrm{AC}$ subsystem is in a balanced state, the $\mathrm{AC}$ side of the bidirectional $\mathrm{AC}$ DC inverter is modeled by the differential equations for three phases shown in equation (8.5): 


$$
L \frac{d i_{a b c}(t)}{d t}+R i_{a b c}(t)=v_{a b c}(t)-u_{a b c}^{g}(t)
$$

After the vector notation, the equation (8.6) can be written in the $a \beta$ stationary frame as:

$$
L \frac{d_{\alpha \beta}(t)}{d t}+R i_{\alpha \beta}(t)=v_{\alpha \beta}(t)-u_{\alpha \beta}^{g}(t)
$$

Equation (8.6) can be written in the rotating reference frame synchronized with grid voltage using Park's transformation such as [159]:

$$
\frac{d i_{d q}(t)}{d t}+(R+j \omega L) i_{d q}(t)=v_{d q}(t)-u_{d q}^{g}(t)
$$

In state space form, the decoupled equation can be written as equation (8.8):

$$
\frac{d X}{d t}=A \cdot X-B \cdot Y
$$

Where the input vector and the state vector are defined as equations (8.9) and (8.10) respectively:

$$
\begin{gathered}
X=\left[\begin{array}{ll}
i_{d} & i_{q}
\end{array}\right]^{T} \\
Y=\left[\begin{array}{llll}
V_{d} & V_{q} & u_{d}^{g} & u_{q}^{g}
\end{array}\right]^{T}
\end{gathered}
$$

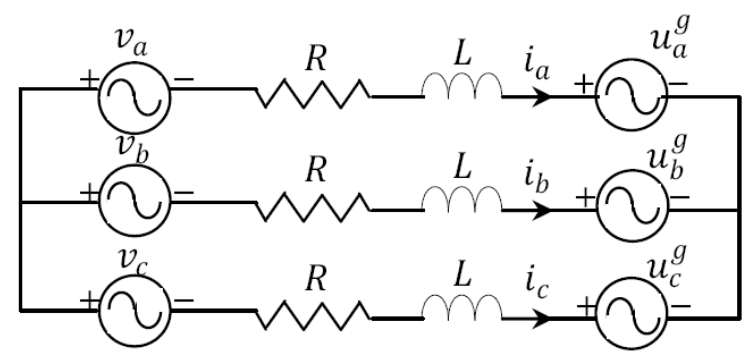

Figure 8.10 Simplified model of the bidirectional AC-DC inverter 
The input matrix and system matrix are given by equations (8.11) and (8.12):

$$
\begin{aligned}
A & =\left[\begin{array}{cc}
-R / L & \omega_{g} \\
-\omega_{g} & -R / L
\end{array}\right] \\
B & =\left[\begin{array}{cccc}
1 / L & 0 & -1 / L & 0 \\
0 & 1 / L & 0 & -1 / L
\end{array}\right]
\end{aligned}
$$

The DC side of the hybrid power system is modeled by equation (8.13):

$$
C_{d c} \frac{d V d(t)}{d t}=i_{d c}(t)-i_{L}(t)
$$

Therefore, the active and reactive power in the synchronous frame is given by equation (8.14):

$$
\begin{aligned}
& P(t)=\frac{3}{2}\left[u_{d}^{g}(t) \cdot i_{d}(t)+u_{q}^{g}(t) \cdot i_{q}(t)\right] \\
& Q(t)=\frac{3}{2}\left[u_{q}^{g}(t) \cdot i_{d}(t)-u_{d}^{g}(t) \cdot i_{q}(t)\right]
\end{aligned}
$$

The power of the DC side in the hybrid power system is given by equation (8.15):

$$
P_{d c}=V_{d c} \cdot i_{d c}
$$

The voltage oriented control method is utilized to control the instantaneous active and reactive power flow through the bidirectional AC-DC inverter by separate controllers that control the $i_{d}$ and $i_{q}$ independently of each other. The control process is as follows. First, the grid voltages and currents are measured. Next, the phase lock loop (PLL) detects the phase angle and frequency of the AC bus to help the bidirectional AC-DC inverter to synchronize with the AC bus. Then the demanded amount of power that needs to be transferred through the inverter is estimated from the AC side with the desired power factor. After that, the three phase reference currents in the synchronous frame 
synchronized with the AC bus are calculated. In the end, the current controllers will regulate the actual currents to the reference values. The reference currents $i_{d}^{r}$ and $i_{q}^{r}$ can be calculated from the following equations (8.16) and (8.17):

$$
\begin{aligned}
& i_{d}^{r}(t)=\frac{2}{3\left(u_{d}^{g 2}(t)+u_{q}^{g 2}(t)\right)}\left[u_{d}^{g}(t) \cdot P^{r}(t)+u_{q}^{g}(t) \cdot Q^{r}(t)\right] \\
& i_{q}^{r}(t)=\frac{2}{3\left(u_{d}^{g 2}(t)+u_{q}^{g 2}(t)\right)}\left[u_{q}^{g}(t) \cdot P^{r}(t)+u_{d}^{g}(t) \cdot Q^{r}(t)\right]
\end{aligned}
$$

where $P^{\prime}(t)$ and $Q^{\prime}(t)$ are the active and reactive power reference values. In a balanced three phase AC system, $u_{q}^{g}(t)$ is equal to zero. Therefore, equations (8.18) and (8.19) can be rewritten as:

$$
\begin{aligned}
& i_{d}^{r}(t)=\frac{2 P^{r}(t)}{3 u_{d}^{g}(t)} \\
& i_{q}^{r}(t)=\frac{2 Q^{r}(t)}{3 u_{d}^{g}(t)}
\end{aligned}
$$

If the system is operated with unity power factor, the reactive power reference is zero, and the current references $i_{d}^{r}$ and $i_{q}^{r}$ can be simplified as equations (8.20) and (8.21):

$$
\begin{aligned}
& i_{d}^{r}(t)=\frac{2 P^{r}(t)}{3 u_{d}^{g}(t)}=\frac{2 \cdot V_{d c} \cdot i_{d c}^{r}(t)}{3 u_{d}^{g}(t)} \\
& i_{q}^{r}(t)=0
\end{aligned}
$$

Based on the state space equation (8.8), the bidirectional output reference voltages can be calculated as: 


$$
\begin{aligned}
& v_{d}^{r}(t)=u_{d}^{j}(t)-\omega \cdot L \cdot i_{q}(t)+R \cdot i_{d}(t)+U_{d}^{v} \\
& v_{q}^{r}(t)=u_{q}^{j}(t)+\omega \cdot L \cdot i_{d}(t)+R \cdot i_{q}(t)+U_{q}^{v}
\end{aligned}
$$

where $U_{d}^{v}$ and $U_{q}^{v}$ are the effective voltage commands which can be calculated by using a PI controller as shown in equations (8.24) and (8.25), and $k_{p}^{d}, k_{i}^{d}$ and $k_{p}^{q}, k_{i}^{q}$ are the proportional and integral parameters of the PI controllers for $i_{d}$ and $i_{q}$, respectively.

$$
\begin{aligned}
& U_{d}^{r}(t)=k_{p}^{d}\left[i_{d}^{r}(t)-i_{d}(t)\right]+k_{i}^{d} \int\left[i_{d}^{r}(t)-i_{d}(t)\right] d t \\
& U_{q}^{r}(t)=k_{p}^{q}\left[i_{q}^{r}(t)-i_{q}(t)\right]+k_{i}^{q} \int\left[i_{q}^{r}(t)-i_{q}(t)\right] d t
\end{aligned}
$$

In this way, the coupling term between the $d-q$ axis is cancelled by the feedforward components $\omega \cdot L \cdot i_{q}(t)$ and $\omega \cdot L \cdot i_{d}(t)$.

The schematic diagram of the grid connected bidirectional AC-DC converter and its decoupled active and reactive power control is shown in figure 8.11.

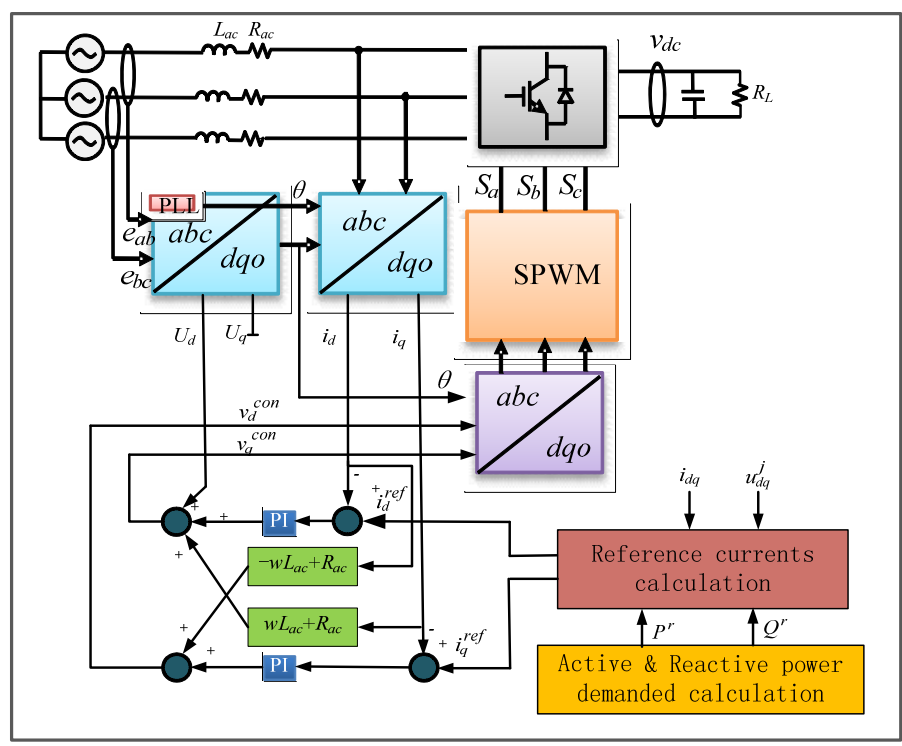

Figure 8.11 Bidirectional AC-DC inverter power control schematic diagram. 
A simulation of the decoupled power control of the bidirectional AC-DC inverter based on MATLAB Simulink is done to analyze the performance of the decoupled active and reactive power control. The simulation block is shown in figure 8.12. The system simulation parameters are shown in Table 8.2.

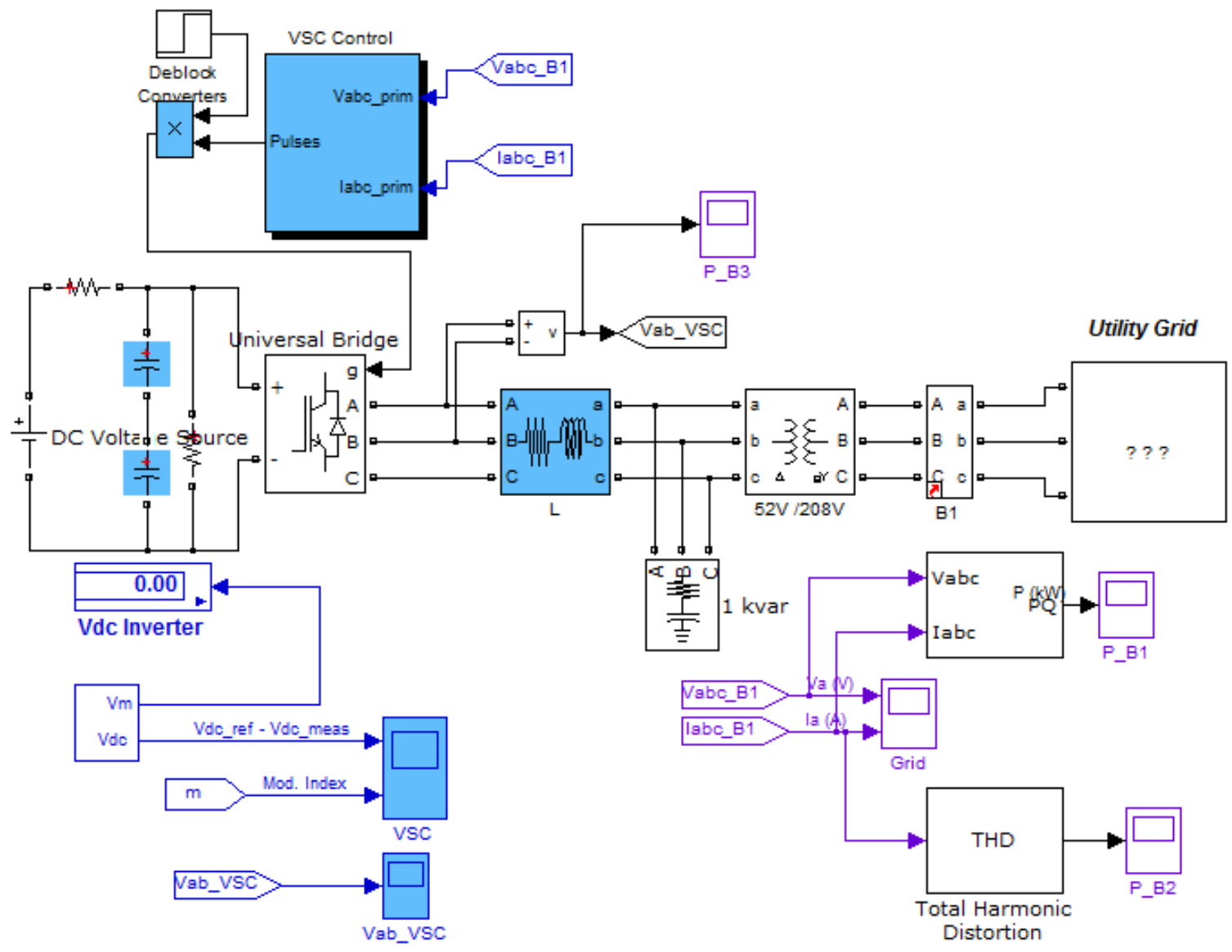

Figure 8.12 Matlab simulation model for bidirectional AC-DC inverter power flow control.

The simulation result is shown in figure 8.13. The initial active power is set as $100 \mathrm{~W}$ and reactive power is set as $0 \mathrm{~W}$. At $\mathrm{t}=0.2$ seconds and 0.4 seconds, the active power reference signal is changed to $300 \mathrm{~W}$ and $-300 \mathrm{~W}$, respectively. At $\mathrm{t}=0.6$ seconds, the active power reference is changed to zero, and the reactive power is set as $200 \mathrm{~W}$. At $t$ 
$=0.8$ seconds, the reactive power is changed from $200 \mathrm{~W}$ to $-200 \mathrm{~W}$. From the simulation we can see the active and reactive power can be independently controlled. Also, the controller can regulate the active and reactive power individually with fast response and small overshot.

\subsubsection{Bidirectional AC-DC inverter frequency and voltage control}

As mentioned before, for bidirectional AC-DC inverter active and reactive power decoupled control, the most important step is to get the reference current signals for id and iq. Since the active and reactive power can be completely decoupled by controlling id and iq, the voltage and frequency controller can be designed by using another control loop to generate the reference signals for id and iq.

To regulate the DC bus voltage, id can be used while at the same time iq is set to zero. The id reference signal can be calculated by using a PI controller as shown in equation (8.26).

$$
i_{d}^{r}(t)=k_{p}^{d c}\left[V_{d c}^{r}(t)-V_{d c}(t)\right]+k_{i}^{d c} \int\left[V_{d c}^{r}(t)-V_{d c}(t)\right] d t
$$

Where $V_{d c}^{r}(t)$ and $V_{d c}(t)$ are the reference and measured DC bus voltage, and $k_{p}^{d c}$ and $k_{i}^{d c}$ are the proportional and integral parameters of the PI controller.

To regulate the AC bus voltage frequency and amplitude, both $i_{d}$ and $i_{q}$ need to be controlled. Active power imbalance will cause AC side frequency variations and the imbalance of reactive power will cause AC side voltage amplitude variations. Two PI controllers are used separately to control the system frequency. The $i_{d}$ and $i_{q}$ reference signals are calculated by using equations (8.27) and (8.28): 




Figure 8.13 Bidirectional AC-DC converter active and reactive power decoupled control 


$$
\begin{aligned}
& i_{d}^{r}(t)=k_{p}^{d f}\left[f^{r}(t)-f(t)\right]+k_{i}^{d f} \int\left[f^{r}(t)-f(t)\right] d t \\
& i_{q}^{r}(t)=k_{p}^{q v}\left[V_{r m s}^{r}(t)-V_{r m s}(t)\right]+k_{i}^{q v} \int\left[V_{r m s}^{r}(t)-V_{r m s}(t)\right] d t
\end{aligned}
$$

where $f^{r}(t)$ and $f(t)$ are the referenced and measured system frequency, $V_{r m s}^{r}(t)$ and $V_{r m s}(t)$ are the referenced and measured AC side voltage amplitudes, and $k_{p}^{d f}, k_{i}^{d f}, k_{p}^{q v}$ and $k_{i}^{q v}$ are the proportional and integral parameters of the PI controllers.

The errors between the reference signals $i_{d}^{r}(t)$ and $i_{q}^{r}(t)$ and the measured $i_{d}(t)$, $i_{q}(t)$ are sent to the inner PI control loop as shown in figure 8.11. The outputs from the inner PI controllers are used to calculate $v_{d}^{r}(t)$ and $v_{q}^{r}(t)$. After converting back to three phase, the bidirectional AC-DC inverter AC side output reference voltages $v_{a}^{r}(t), v_{b}^{r}(t)$,

Table 8.2 Specifications and parameters for Bidirectional AC-DC Inverter Active and Reactive Power Decoupled Control Simulation

\begin{tabular}{ccc}
\hline \hline Symbol & Quantity & Value \\
\hline$f_{g}$ & AC subsystem frequency & $60 \mathrm{~Hz}$ \\
$V_{d c}$ & DC bus voltage & $120 \mathrm{~V}$ \\
$V_{g}$ & Grid voltage & $208 \mathrm{~V} r . m . s$ \\
$n$ & Transformer ratio & $4: 1$ \\
$C_{d c}$ & DC bus capacitance & $6000 \mu f$ \\
$L_{f}$ & RL filter inductance & $12 \mathrm{mH}$ \\
$R_{f}$ & RL filter resistance & $0.3 \Omega$ \\
$f_{s}$ & Switching frequency & $6 \mathrm{kHz}$ \\
\hline \hline
\end{tabular}


and $v_{c}^{r}(t)$ can be calculated. Those three phase reference voltage signals are first normalized and then compared with a high frequency saw-tooth waveform to generate the PWM signals, which finally will be used to drive the six IGBTs in the bidirectional ACDC inverter.

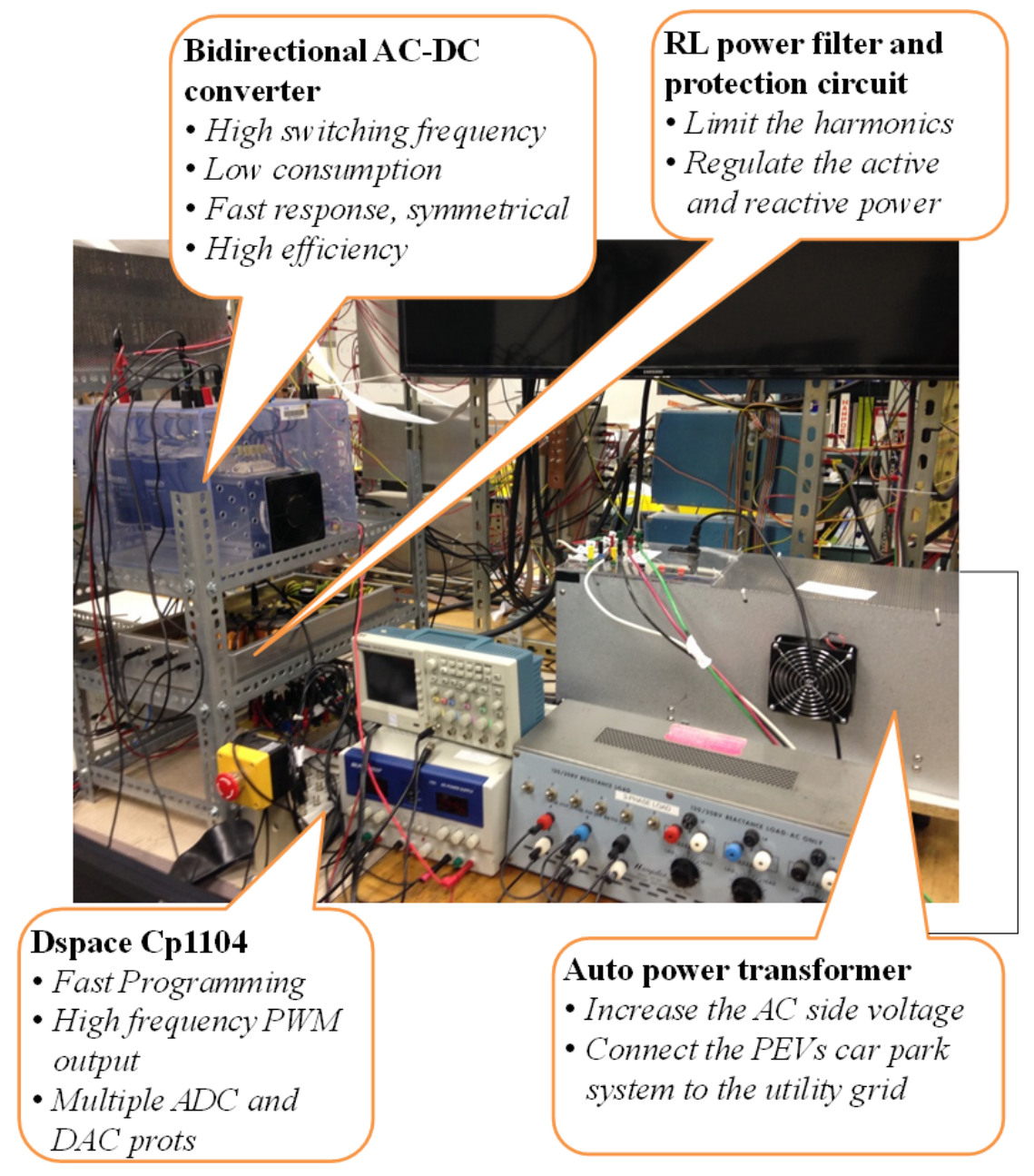

Figure 8.14 Bidirectional three phase AC-DC inverter hardware setup

\subsubsection{Bidirectional AC-DC inverter hardware}

The hardware setup of the three phase bidirectional AC-DC inverter together with the RL filter, driver circuit, and measurement circuit is shown in figure 8.14. It has a three phase bidirectional AC-DC inverter with $6 \mathrm{~kW}$ rated power. The transformer 
primary side can be connected to the utility grid or the AC side of the test bed in the Florida International University Energy Research Laboratory. A dSpace CP1104 controller is used to control the power flow through the inverter. A RL power filter with $12 \mathrm{mH}$ inductance and $0.3 \Omega$ resistance is used to limit the system harmonics and regulate the active and reactive power.

\subsubsection{Real time experimental verification}

Two hardware experiments were done for verification of the performance of the bidirectional AC-DC inverter. The first experiment shows the bidirectional active and reactive power between flow the AC and DC subsystems in the hybrid power system. In this experiment, the hybrid power system is working in grid-connected mode and the active and reactive power flow through the bidirectional AC-DC inverter is controlled by regulating the $I_{d}$ and $I_{q}$ respectively. The AC side phase-to-phase voltage is $208 \mathrm{~V}$ RMS with a frequency of $60 \mathrm{~Hz}$. The DC side voltage is $120 \mathrm{~V}$. The transformer between the $\mathrm{AC}$ bus and bidirectional AC-DC inverter reduces the phase-to-phase voltage to $52 \mathrm{~V}$ RMS.

The initial $I_{d}$ reference was set as $-3 \mathrm{~A}$ and $I_{q}$ reference was set as $0 \mathrm{~A}$, which means the DC subsystem was injecting active power to the AC subsystem with unity power factor. At $t=3.1$ seconds, the $I_{d}$ reference changed to $3 \mathrm{~A}$ and $I_{q}$ reference stayed the same. The $I_{d}, I_{q}$ control performance is shown in figure 8.15 (a). The $I_{d}$ measured current could closely follow the reference signal, and reach the steady state in less than 0.5 seconds. Also, the $I_{q}$ was not influenced by the variation of $I_{d}$. The phase A voltage and current transient response is shown in figure 8.15 (b). The current phase shifted 180 degree in one cycle. 
The $I_{q}$ control was also tested and shown in figure 8.16 (a). The initial $I_{q}$ reference was set as $-3 \mathrm{~A}$ and $I_{d}$ reference was set as $0 \mathrm{~A}$, which means the DC subsystem was injecting reactive power to the AC subsystem for reactive power compensation. At $t=2.8$ seconds, the $I_{q}$ reference changed to $3 \mathrm{~A}$ and $I_{d}$ reference stayed the same. The $I_{q}$

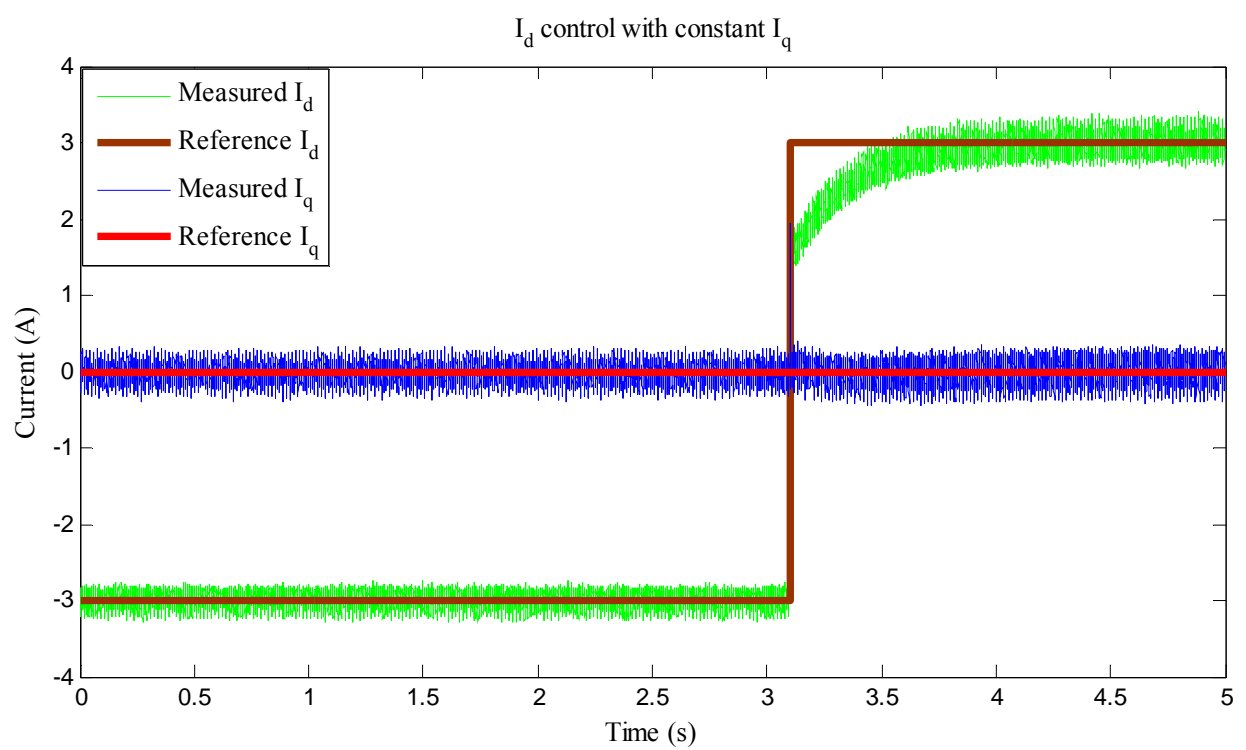

(a)

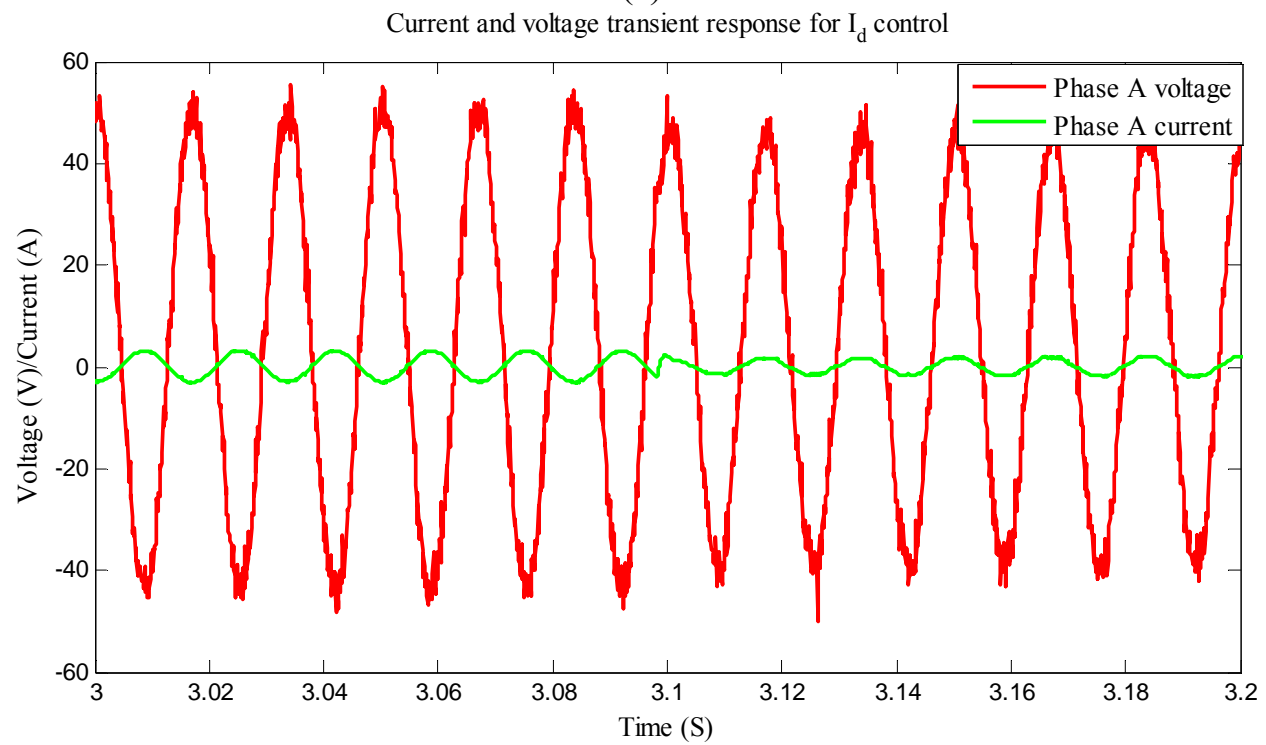

(b)

Figure 8.15 Bidirectional AC-DC inverter hardware active power control. (a) $I_{d}$ control (b) phase A voltage and current response. 
measured current could closely follow the reference signal, and reached the steady state in less than 0.5 seconds. Also, the $I_{q}$ was not influenced by the variation of $I_{q}$. The phase A voltage and current transient response is shown in figure 8.16 (b). The current phase shifted 180 degree in one cycle.

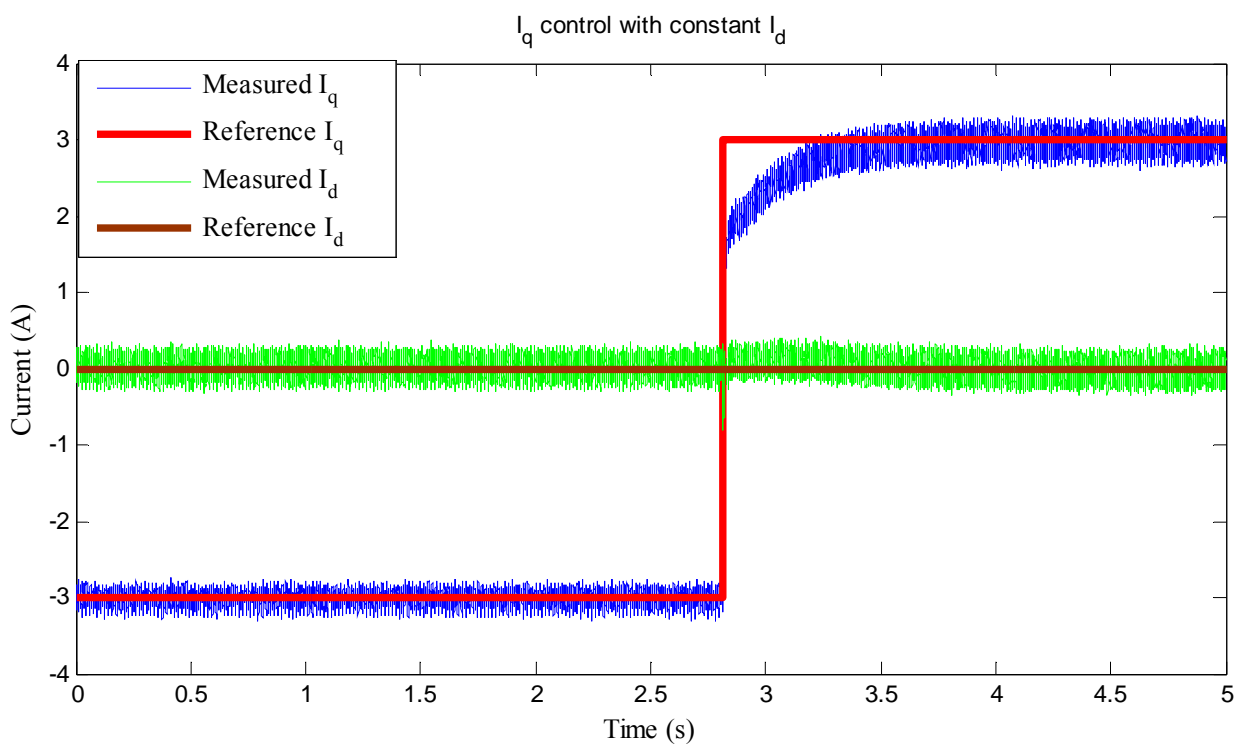

(a)

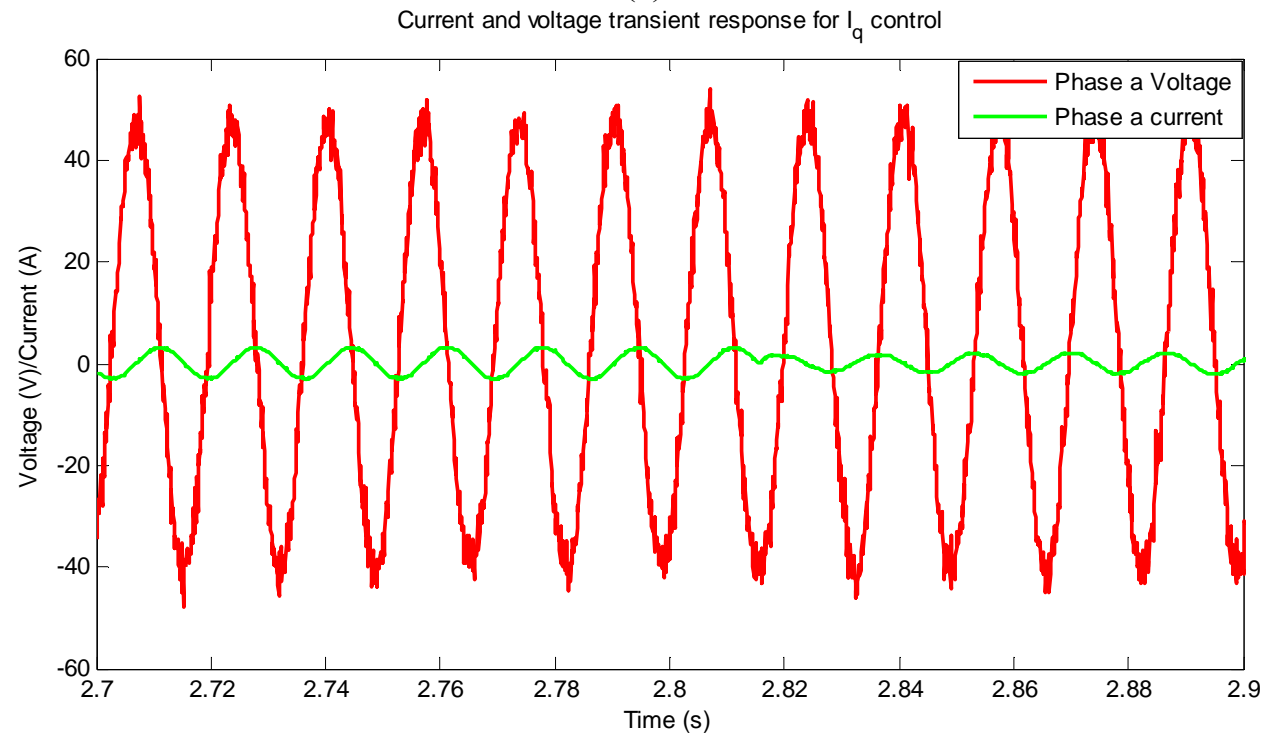

(b)

Figure 8.16 Bidirectional AC-DC inverter hardware reactive power control. (a) $I_{q}$ control (b) phase A voltage and current response. 
The second experiment was done to verify the bidirectional AC-DC inverter frequency regulation function. In this experiment, the hybrid power system is operated in islanding mode with a $0.6 \mathrm{~kW}$ load on the AC subsystem. Also, the AC side has an induction generator operated in power-voltage control mode with $0.4 \mathrm{~kW}$ constant output power. The system frequency response is shown in figure 8.17. There is no slack generator in the hybrid power system; therefore the initial system frequency was around 58.3Hz. The bidirectional AC-DC inverter frequency regulation was enabled at $\mathrm{t}=$ 10:38:40, and the system frequency immediately increased and stabled at $60 \mathrm{~Hz}$.

After that, the system frequency is controlled to reach at most $60.5 \mathrm{~Hz}$, at least $59 \mathrm{~Hz}$, and back to $60 \mathrm{~Hz}$. In the end, the frequency regulation function is disabled, and the

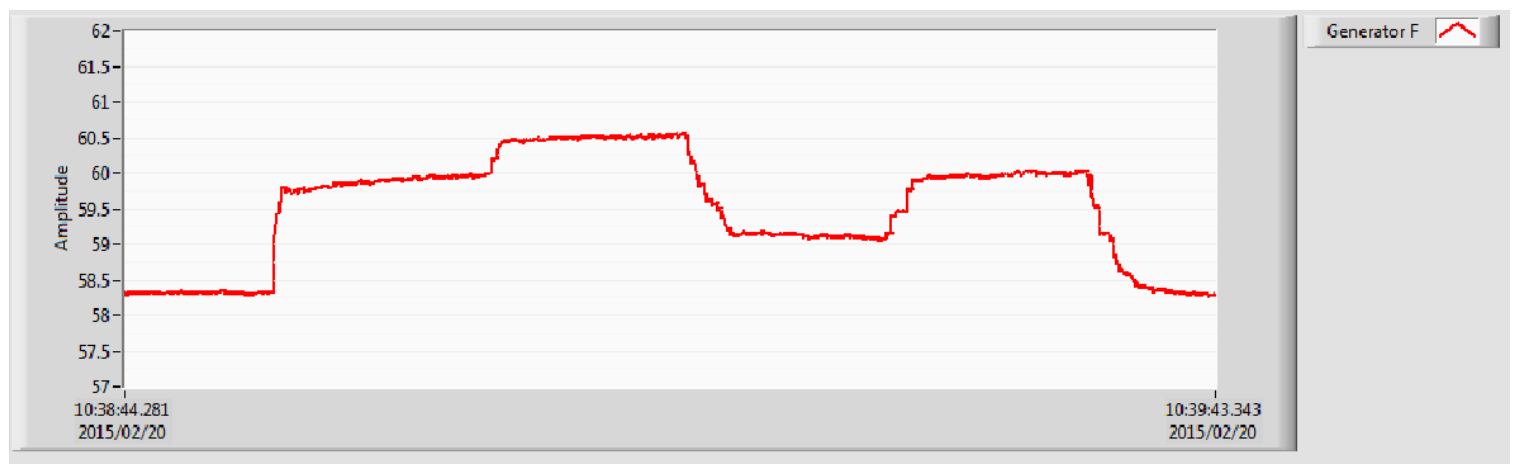

Figure 8.17 Hybrid AC-DC power system AC subsystem frequency

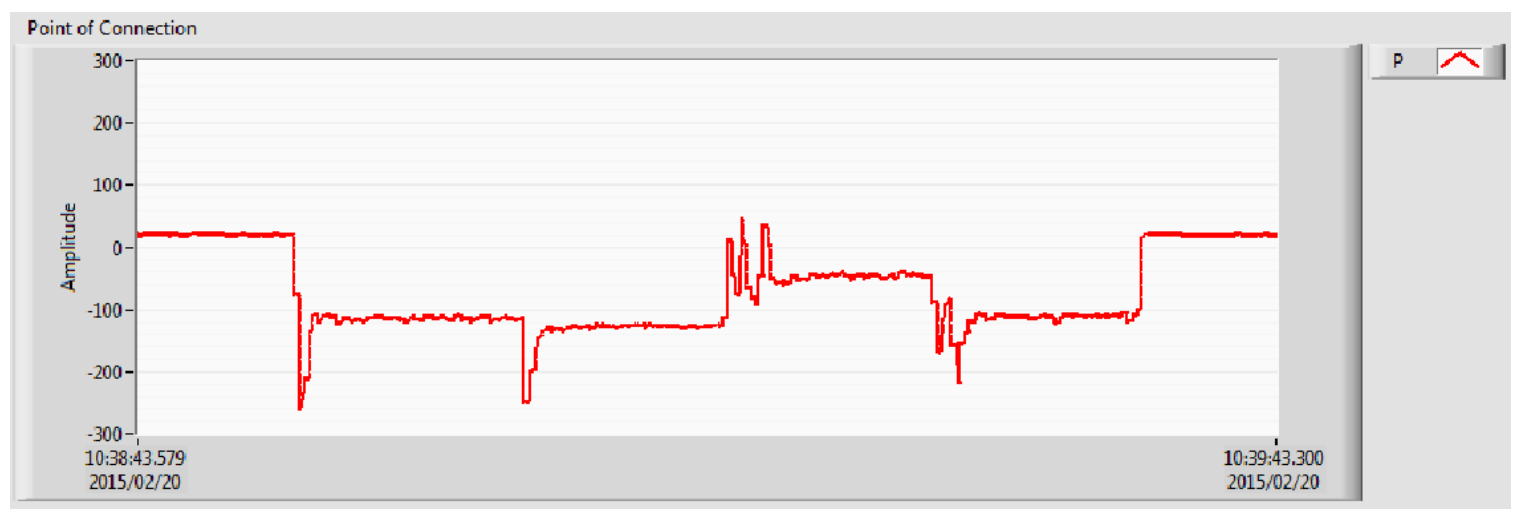

Figure 8.18 Power injected from DC subsystem to AC subsystem 
system frequency dropped back to $58.3 \mathrm{~Hz}$. The power injected from the DC subsystem to the AC subsystem is shown in figure 8.18. From the experimental results it can be seen that the hybrid AC-DC power system AC subsystem frequency can be fully controlled by the bidirectional AC-DC inverter when it is operated in islanding mode.

\subsection{Hybrid Power System Performance with Mitigation of Critical Loads}

\subsubsection{Maximizing power generation from photovoltaic panel emulator}

In this chapter, the perturbation and observe $(\mathrm{P} \& \mathrm{O})$ method was used to track the maximum power point. The algorithm utilizes the PV farm output current and voltage to calculate the power. The values of the voltage and power at the $k^{\text {th }}$ iteration $\left(P_{k}\right)$ are stored, then the same values are measured and calculated for the $(k+1)^{\text {th }}$ iteration $\left(P_{k+1}\right)$. The power difference between the two iterations $(\Delta P)$ is calculated. The converter should increase the PV panel output voltage if $\Delta P$ is positive and decrease the output voltage if $\triangle P$ is negative, which finally will adjust the duty cycle. The PV panel reaches the maximum power point when $\Delta P$ is approximately zero. The detailed description of $\mathrm{P} \& \mathrm{O}$ MPPT methods together with its flowchart is shown in Chapter 3 section 3.3.3.

To maximize the utilization of renewable energy from the PV farm, the boost converter should be operated in on-MPPT mode when the hybrid power system is connected to the utility grid. The battery banks or the PEVs in the power system can be used as an energy buffer, and the charging/discharging rates can be controlled based on the status of the output power from the PV farm and the power flow on the AC side. In islanding mode, the boost converter of the PV farm can be operated in on-MPPT or offMPPT, depending on the system's power balance and the SOCs of the battery banks. In 
most situations, this boost converter can operate in the on-MPPT mode since the variation ratio of the solar irradiance is much slower compared with the power adjustment ability of the small size AC generator. Therefore, for a constant load either on the AC or DC side, the PV should supply as much power as possible to maximize its utilization. However, if the battery banks' SOCs are high (near fully charged) and the PV's maximum output power is larger than the total load in the hybrid power system, the PV should be turned to off-MPPT to keep the system power in balance.
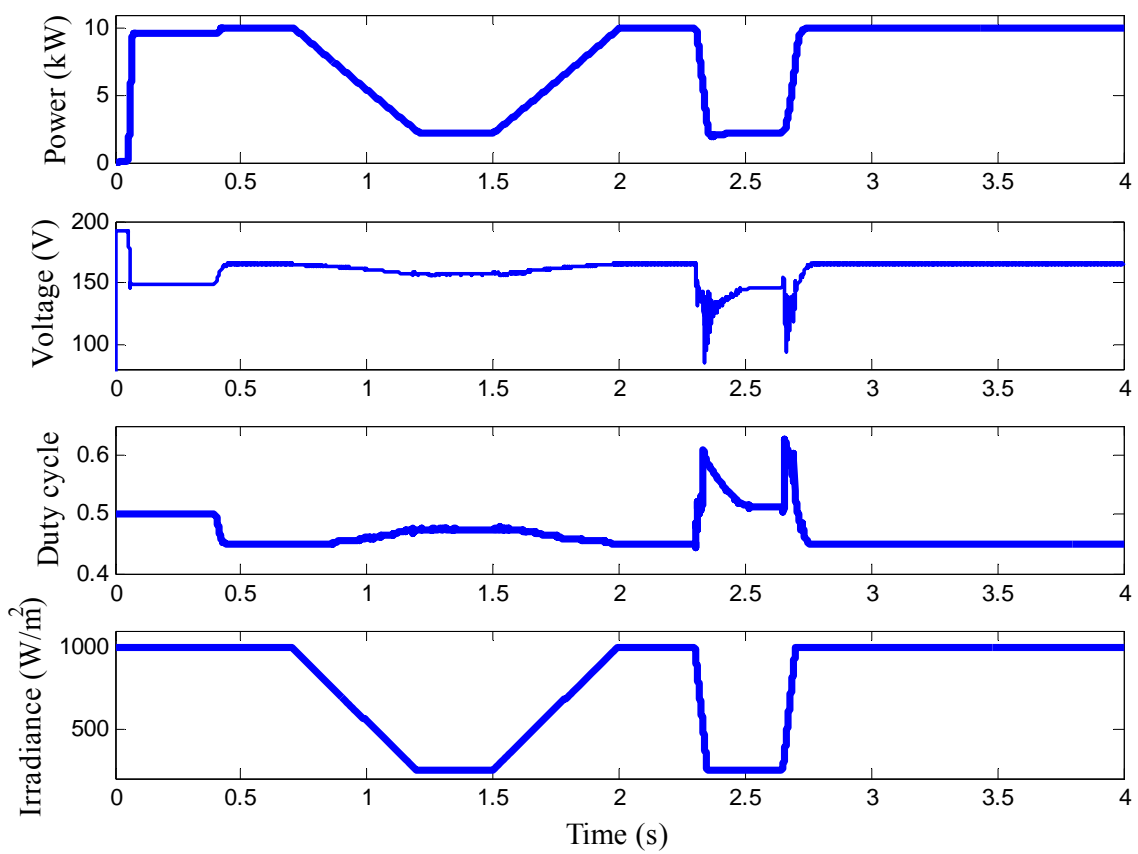

Figure 8.19 PV farm output power control with MPPT.

In both grid-connected mode and islanding mode, to maximize the utilization of renewable energy, the boost converter is working in on-MPPT mode to keep seeking the maximum output power from the PV farm. The MPPT of the boost converter is enabled at $\mathrm{t}=0.4$ seconds. The output power, the terminal voltage of the PV panel, the duty cycle of the boost converter, and the solar irradiance are shown in figure 8.19. For general study, two kinds of solar irradiance variances with different charging rates were used in 
this study. Before $t=0.4$ seconds, the duty cycle is set at 0.5 , the terminal voltage of the PV panel is $149 \mathrm{~V}$, and the output power from the PV panel is only $9.56 \mathrm{~kW}$. After the MPPT is enabled, the duty cycle is decreased to 0.45 and the terminal voltage is increased to $165 \mathrm{~V}$. This allows the PV panel to reach the maximum power output of $10.07 \mathrm{~kW}$. The simulation results show that the boost converter with MPPT functionality can track the maximum power point within a short response time.

\subsubsection{Power flow in the hybrid AC-DC power system}

The bidirectional DC-DC converters are used to connect the battery banks to the DC bus. In grid-connected mode, those converters only regulate the battery banks (or PEVs) charging rates. Based on the SOCs of the battery banks and the power flow conditions on the $\mathrm{AC}$ side, the charging/discharging current references are generated to regulate the current flow in the converters. Each battery has its own bidirectional DC-DC converter, which means they can have different charging rates. By doing this, the battery banks can inject power to or absorb power from the DC bus, and even transfer energy between different battery banks if necessary. In this case, one current control closed loop with PI controller is enough to regulate the current.

The bidirectional DC-DC converters of the battery banks play an important role in islanding mode to regulate the DC bus voltage. A two-loop control is used to regulate the DC bus voltage. The control scheme for the bidirectional DC-DC converter is shown in figure 8.20. The outer voltage controlled loop is used to generate a reference charging current for the inner current controlled loop. The error between the measured DC bus voltage and the system reference DC bus voltage is set as the input of the PI controller,

and the output is the reference current. The inner current control loop will compare the 
reference current signal with the measured current flow through the converter and finally generate a PWM signal to drive the IGBTs to regulate the current flow through the converter. For example, when the DC bus voltage is higher than the reference voltage, the outer voltage controller will generate a negative current reference signal, and the inner current control loop will adjust the duty cycle to force the current flow from the DC bus to the battery, which results in charging of the battery. In this way, the energy transfers from the DC bus to the battery and the DC bus voltage will decrease to the rating value. If the DC bus voltage is lower than the normal value, the outer voltage control loop will generated a positive current reference signal, which will regulate the current flow from the battery to the DC bus, and because of the extra energy injected from the batteries, the DC bus voltage will increase to the rating value. All five batteries and their bidirectional converters are coordinated together to regulate the DC bus voltage. The battery SOC and thermal self-balancing control is also applied to protect the battery banks from over discharge and overheating.

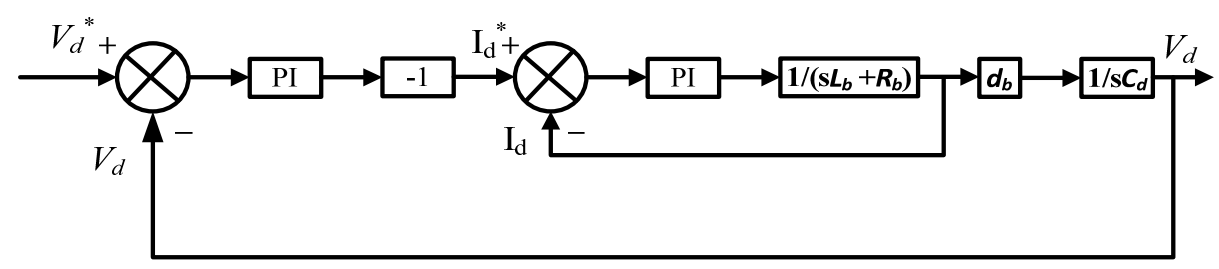

Figure 8.20 Voltage control block diagram for bidirectional DC/DC converter In grid connected mode, the AC side can be viewed as an infinite bus. Therefore, the variances of the voltage amplitude and frequency can be ignored. In this case, the bidirectional AC-DC converter only needs to regulate the DC bus voltage. To operate at unity power factor, reference $I_{q}$ can be set as 0 . The controller only needs to control the 
$I_{d}$, which in the end will control the active power flow through the converter. What's more, if the grid needs the hybrid power system to provide ancillary services, such as AC grid frequency regulation, the proposed configuration is also qualified. For example, if the system frequency is below $60 \mathrm{~Hz}$, the central control aggregator will send a command to the bidirectional DC-DC converter to let the energy storage systems inject power to the DC bus, which will result in an increase in the DC bus voltage. The bidirectional AC-DC converter will sense this voltage increase and will decrease the current reference, $i_{d}$. This decrease in current reference will result in the decrease of active power flow from the $\mathrm{AC}$ side to the DC side of the hybrid power system. If $I_{d}$ decreases to a negative value, then energy will be transferred from the DC side to the AC side. In the end, the power is balanced on the $\mathrm{AC}$ side, and the frequency will increase back to $60 \mathrm{~Hz}$. The control block diagram for the bidirectional AC-DC converter in grid-connected mode is shown in figure 8.21. A two-loop controller is used to regulate the DC bus voltage. Based on the error between the DC bus reference voltage and measured voltage, the outer voltage control loop generates the $I_{d}$ reference which is used to regulate $I_{d}$ in the bidirectional converter. The two-loops controller will regulate the DC bus voltage by transferring active power between the AC and DC subsystems in the hybrid AC-DC power system. During the regulation, the reactive power is limited to zero, which will make the DC subsystem act as a pure resistance load with unity power factor. Under this operation, the DC micro grid will not consume reactive power, therefore the voltage amplitude of the AC side of bidirectional AC-DC inverter will not drop and doesn't need reactive power compensation. 


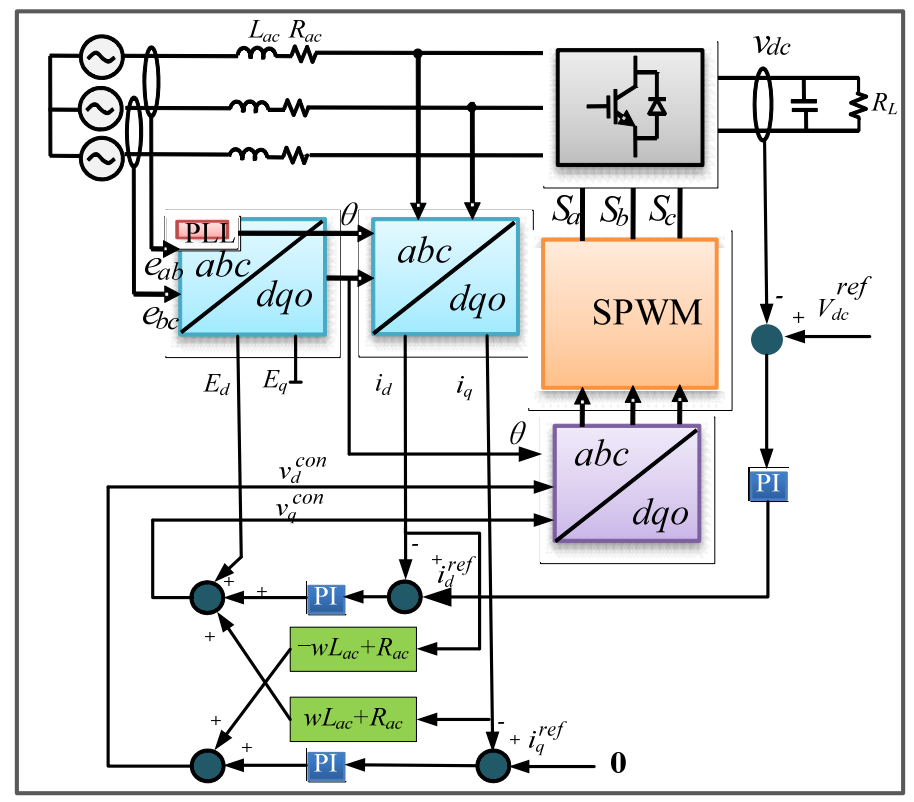

Figure 8.21 DC bus voltage control block diagram for bidirectional AC-DC inverter

In islanding operation mode, the frequency and voltage amplitude of the three phase AC side are not so robust, and regulations are needed. Usually, the slack generator in the hybrid power system can handle the load with a small varying ratio, but when the pulse load is connected to the AC side or the total load exceeds the generator's limit, the system frequency may decrease and the voltage may collapse. Therefore, the bidirectional AC-DC inverter is used to help regulate the AC side frequency and voltage amplitude. The control scheme for the bidirectional AC-DC inverter is shown in figure 8.22. Two-loop controllers are applied for both frequency and voltage regulation. For frequency control, the error between measured frequency and reference frequency is sent to a PI controller which generates the $I_{d}$ reference. To control the voltage amplitude, the error between the measured voltage amplitude and the reference voltage amplitude is sent to a PI controller to generate $I_{q}$ reference. The DC bus voltage is regulated by the energy storage system and bidirectional DC-DC converters. 




Figure 8.22 AC bus voltage control block diagram for bidirectional AC-DC inverter

\subsubsection{Hybrid AC-DC power system simulation verification under pulse load}

To verify the proposed control method for the hybrid AC-DC power system operated in grid-connected mode, two simulations about the proposed hybrid AC-DC system operated in grid-connected and islanding mode were done. The operations of the hybrid AC-DC power system utilizing a $10.07 \mathrm{~kW}$ PV farm under the influence of a 10 $\mathrm{kW}$ pulse load was simulated. The rated power of the synchronous generator is $13.8 \mathrm{~kW}$, and a $4 \mathrm{~kW}$ constant load is connected to the AC side. Five 51.8V 21Ah Lithium-ion battery banks are connected individually to the DC bus through bidirectional DC/DC converters.

In grid-connected mode, the DC bus voltage is regulated by the bidirectional ACDC converter. In this mode, the AC side can be viewed as an infinite bus, the $10 \mathrm{~kW}$ resistive pulse load that is connected to the AC side will not have an influence on the 
grid. Therefore, only the DC pulse load case is studied. The DC bus voltage with the influence of the PV output power variance and battery banks (PEVs) charging rate variance is shown in figure 8.23. Figure 8.23 (a) shows the DC bus voltage. The bidirectional AC-DC converter was enabled at $\mathrm{t}=0.05$ seconds. Before that, it operated as an uncontrolled rectifier. After enabling the bidirectional converter, the DC bus voltage reached steady state in less than 0.3 seconds. During this period, the solar irradiance was $1 \mathrm{~kW} / \mathrm{m}^{2}$. From 0.4 seconds to 1.7 seconds, the system was simulated under two kinds of solar irradiance variations. The output power from the PV farm decreased from $10 \mathrm{~kW}$ to $2.5 \mathrm{~kW}$ in 0.3 seconds and recovered back from $2.5 \mathrm{~kW}$ to $10 \mathrm{~kW}$ also in 0.3 seconds. After that, the PV farm output decreased from $10 \mathrm{~kW}$ to $2.5 \mathrm{~kW}$ in 0.05 seconds at $\mathrm{t}=1.3$ seconds and went back from $2.5 \mathrm{~kW}$ to $10 \mathrm{~kW}$ at $\mathrm{t}=1.65$ seconds. The PV farm output power is shown in figure 8.23 (b). The DC bus voltage was stable and kept in the range of $293 \mathrm{~V}$ to $307 \mathrm{~V}$ during this process. The bidirectional AC-DC inverter can keep the DC side stable under rapid alteration of solar irradiance and PV farm output power. From $t=2$ seconds to 2.8 seconds, the charging/discharging of the battery banks impact to the DC bus was simulated. This process can be viewed as the study of the PEVs charging process in reference [59]. At $t=2$ seconds, the current references of the bidirectional DC-DC converters of those five batteries were changed from $0 \mathrm{~A}$ to $-4 \mathrm{~A}$, which means discharging those battery banks with $4 \mathrm{~A}$. At $\mathrm{t}=2.4$ seconds, the current references of the bidirectional DC-DC converters of the five battery banks were changed from $-4 \mathrm{~A}$ to $4 \mathrm{~A}$, and the system began charging the battery banks. The current flow of one bidirectional DC-DC converter is shown in figure 8.23 (c). During this period, the DC bus voltage was still stable with less than $3 \mathrm{~V}$ voltage variance. 
From $\mathrm{t}=3$ seconds to 3.5 seconds, a $10 \mathrm{~kW}$ resistive load was connected to the DC bus. During the connection and disconnection of the $10 \mathrm{~kW}$ pulse load, the bidirectional ACDC inverter active power flow was greatly changed to regulate the DC bus voltage. The DC bus was still stable within $12 \mathrm{~V}$ voltage variance during the transient response.

The power flow through the bidirectional AC-DC inverter is shown in figure 8.23 (d). After the system entered steady state, the system kept unity power factor as the reactive power was 0 . The active power flow varied with the solar irradiance influence, battery banks charging/discharging influence, and pulse load influence. The bidirectional inverter can quickly adjust the power flow through it and keep the hybrid AC-DC power system stable.

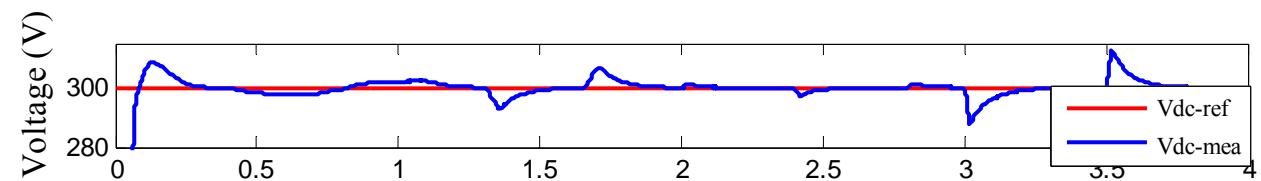

(a)

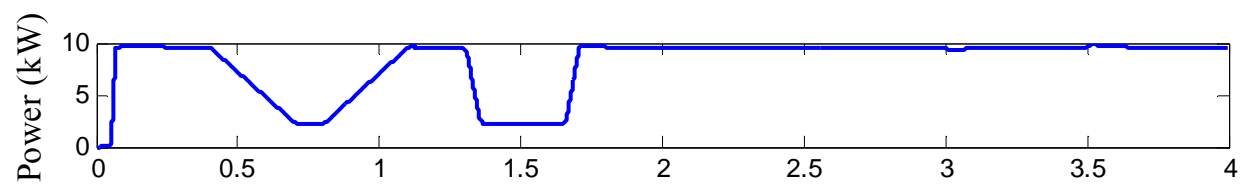

(b)

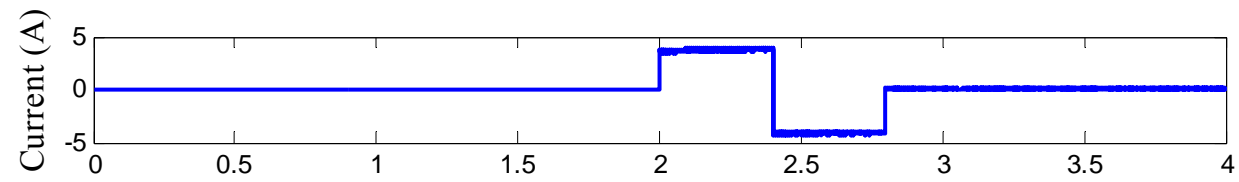

(c)

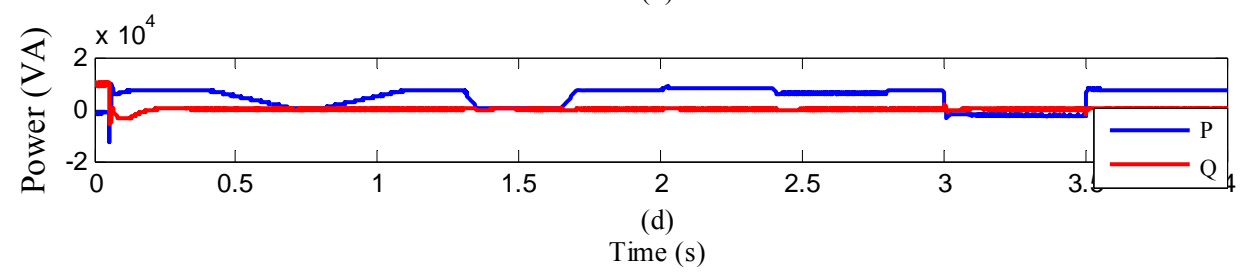

Figure 8.23 Hybrid AC-DC power system performances in grid-connected mode. 
In islanding mode operation, the frequency and voltage amplitude need to be regulated. Usually, the slack generator in the hybrid AC-DC power system can handle the load with a small varying ratio, but when the pulse load is connected to the AC side or the total load exceeds the generator's limit, the system frequency may decrease and the voltage may collapse. Therefore, the bidirectional AC-DC inverter was used to regulate the AC side frequency and voltage amplitude. In this case of study, the AC side had a $4 \mathrm{~kW}$ constant resistive load, and a $10 \mathrm{~kW}$ pulse resistive load connected from $\mathrm{t}=2.2$ seconds to 2.8 seconds. After that, a $10 \mathrm{~kW}$ pulse resistive load was connected to the DC bus from $\mathrm{t}=3.3$ seconds to 3.8 seconds.

Figure 8.24 (a) shows the DC bus voltage with the influence of solar irradiance


Figure 8.24 Hybrid AC-DC power system performances in grid-connected mode 
variation on the PV panels and the pulse load connected on the AC side and DC side. The bidirectional DC-DC converter was enabled at $\mathrm{t}=0.05$ seconds to regulate the DC bus voltage, and the DC bus voltage reached the steady state in less than 0.2 seconds. The PV farm output power variance is shown in figure 8.24 (b). When the $10 \mathrm{~kW}$ pulse load is connected to the $\mathrm{DC}$ bus at $\mathrm{t}=3.3$ seconds, those five battery banks cooperated together to regulate the voltage, therefore the DC bus voltage kept stable with a maximum variance of $17 \mathrm{~V}$ during the transient response. The current follow through one bidirectional DC-DC converter is shown in figure 8.24 (c). The AC side pulse load connection and disconnection did not greatly impact the DC bus voltage since the battery banks have enough energy to support and balance the power flow with a quick response. The bidirectional AC-DC inverter successfully transferred the energy from the battery banks to the AC side when the pulse load happened. The active and reactive power flow through the bidirectional AC-DC inverter is shown in figure 8.24 (d).
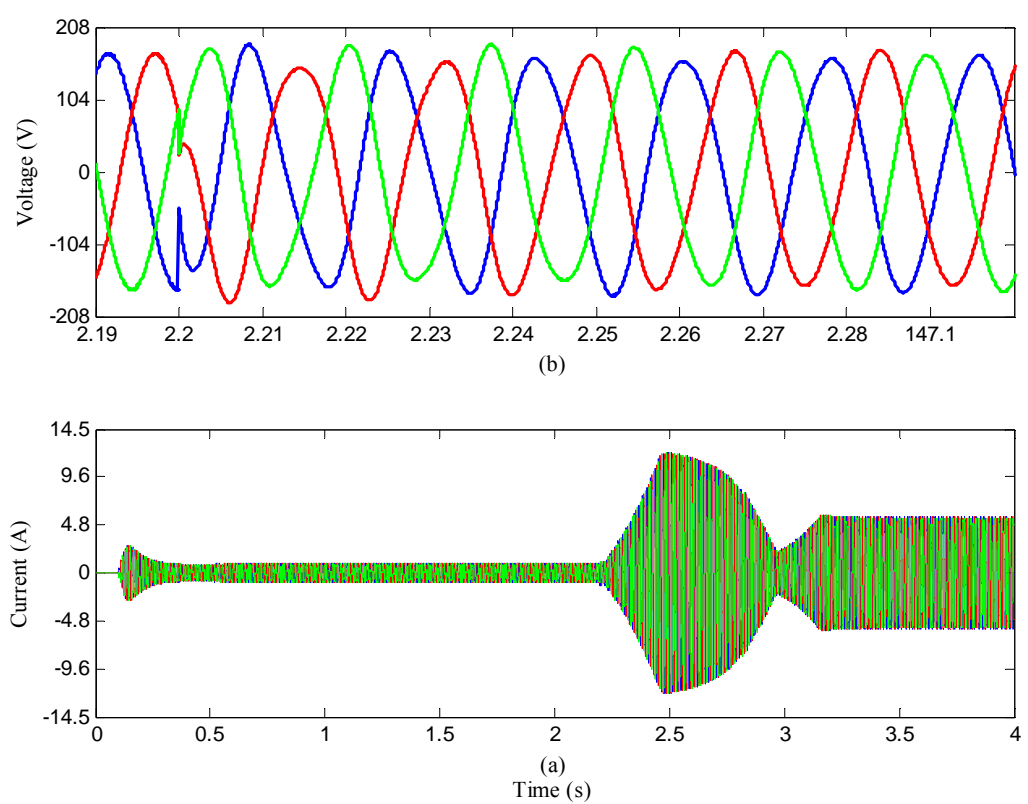

Figure 8.25 Microgrid AC bus voltage and current response with DC support. 
Figure 8.25 (a) and (b) show the AC bus voltage and current. The AC bus voltage transient response during the pulse load connection is shown in figure 8.25 (a). The AC voltage amplitude returned to its normal value in less than three cycles. When the pulse load was connected to the $\mathrm{AC}$ side, the current flow through the $\mathrm{AC}$ bus increased immediately, and after the pulse load disconnected from the AC side, the current slightly decreased to keep the system in balance. The current flow through AC bus is shown in figure 8.25 (b).

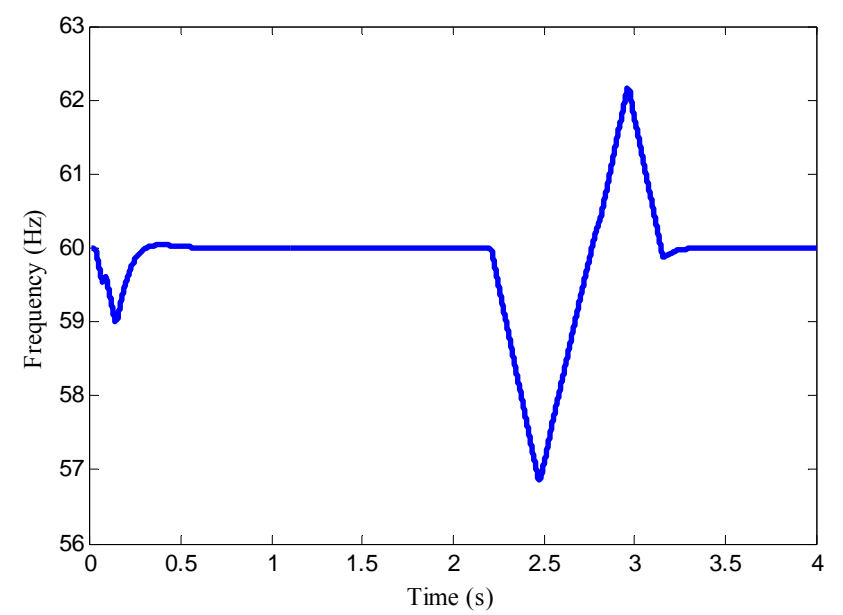

(a)

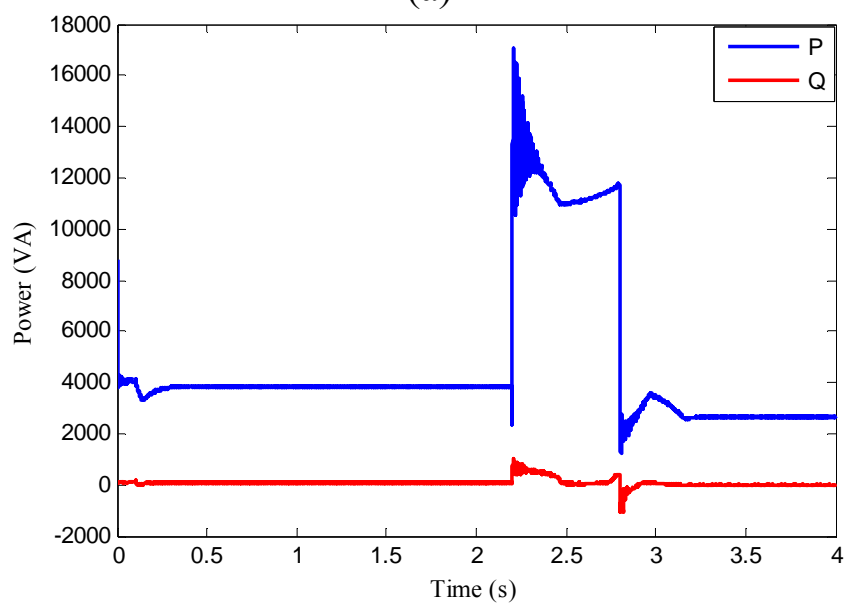

(b)

Figure 8.26 Hybrid AC-DC power system AC subsystem pulse load response with DC support. 


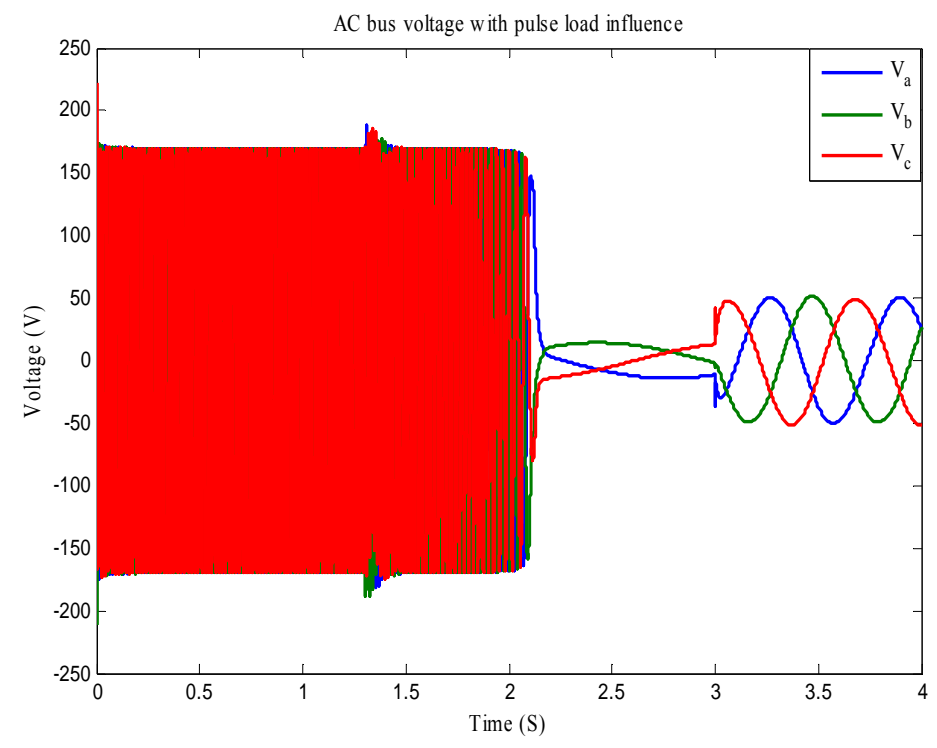

(a)

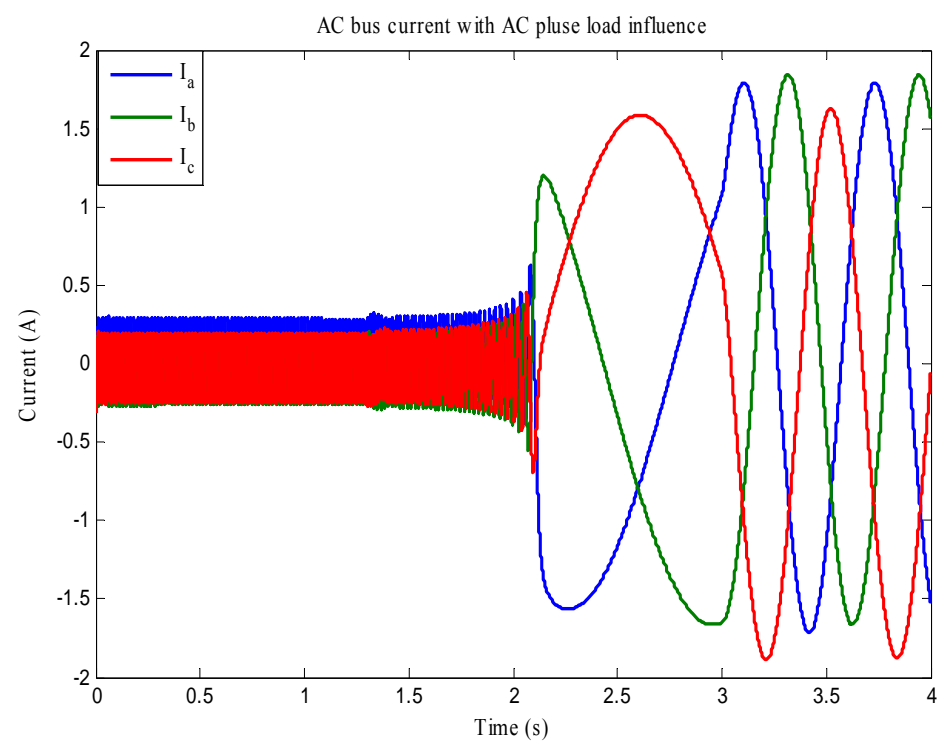

(b)

Figure 8.27 AC subsystem slack generator performance under pulse load (a) output voltage, (b) output current.

Figure 8.26 (a) shows the AC side frequency variation. The bidirectional AC-DC inverter was enabled at $\mathrm{t}=0.1$ seconds and the $\mathrm{AC}$ side frequency became stable at $60 \mathrm{~Hz}$ in less than 0.4 seconds. When the resistive pulse load was connected at $t=2.2$ seconds, the frequency dropped to $58 \mathrm{~Hz}$ and returned to $60 \mathrm{~Hz}$ in less than 1 second. When the 


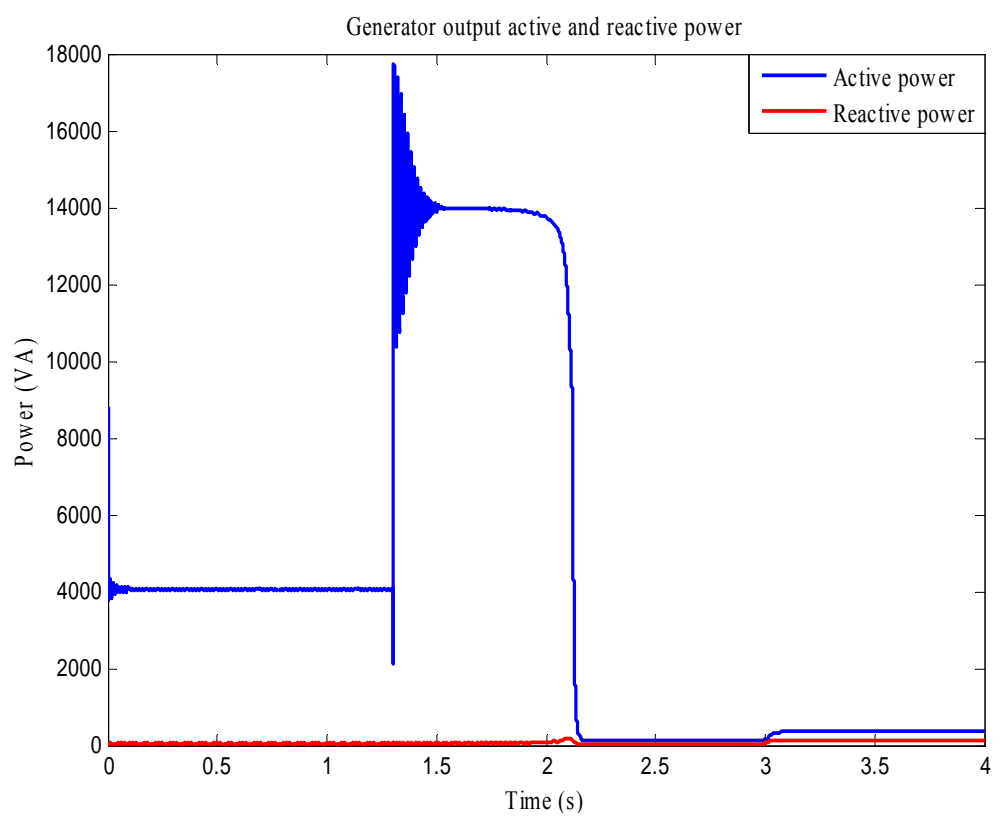

Figure 8.28 AC subsystem frequency response

pulse load was disconnected from the $\mathrm{AC}$ side, the frequency increased to $62 \mathrm{~Hz}$ and returned to stead state in less than 0.5 seconds. The power generated from the generator is shown in figure 8.26 (b). The hybrid AC-DC power system is stable on both its AC and DC sides.

For comparison, another simulation was done for the hybrid micro grid under the same islanding mode operation without DC side support. When the $10 \mathrm{~kW}$ resistive pulse load was connected to the $\mathrm{AC}$ bus, the total load on the $\mathrm{AC}$ side was $14 \mathrm{~kW}$ which exceeded the generator's output limitation by $0.2 \mathrm{~kW}$. Figure 8.27 (a) and (b) shows the AC subsystem generator output voltage and current.

The AC subsystem frequency response is shown in figure 8.28. The pulse load was connected to the $\mathrm{AC}$ side at $\mathrm{t}=2$ seconds, after 0.2 seconds the system collapsed, and both the frequency and voltage dropped considerably. The system couldn't recover even when the pulse load was disconnected after $\mathrm{t}=3$ seconds. 


\subsection{Conclusion}

In this section, a coordination power flow control method for multiple power electronic devices is proposed for a hybrid AC-DC power system operated in both gridconnected and islanding modes. The hybrid power system has a PV farm and two local generators that supply energy to its DC and AC sides. Battery banks are connected to the DC bus through a bidirectional DC-DC converter. The AC side and DC side are linked by the bidirectional AC-DC inverter. The system topology together with the control algorithms under both modes was tested with the influence of pulse loads and renewable energy farm output power variances. The simulation and hardware experimental results show that the proposed hybrid AC-DC power system with the control algorithm can greatly increase the system stability and robustness while simultaneously improving the system frequency and energy harvesting from renewable energy sources. 
9. Application of Microcontrollers and Wireless Transmitters for Monitoring and Control of Hybrid AC-DC Power Systems

\subsection{Introduction}

The implementation of hybrid AC-DC power systems, along with the utilization of plug-in electric vehicles (PEVs) are growing in popularity, increasing the demand for efficient and reliable power flow control systems that can monitor and regulate power flow in hybrid AC-DC power systems [34],[160]. Control of power flow in hybrid ACDC power systems is done by controlling the multiple bidirectional DC-DC and AC-DC converters used in these grids [152]. It is of the utmost importance to design algorithms for communication between devices, such as converters and battery management systems, to monitor and smartly control the power flow across hybrid AC-DC power systems [161]. The growing implementation of smart grids and wireless communications emphasizes the need to implement wireless communication devices with applications to power flow control.

As mentioned in Chapter 7, the future PEVs can be cooperated as a network, whose charging process can be monitored and smartly distributed. In this way, the charging impacts of the PEVs will be greatly limited and the PEV network can be used to improve the utility grid performance through vehicle to grid services (V2G). Therefore, it is necessary to build a PEV car park emulator hardware platform where PEV state monitor and smart charging algorithms can be tested. 
In this chapter, a methodology of establishing a grid connected hybrid PEV car park emulator system together with its power flow control method by utilizing microcontrollers and wireless transceivers to wirelessly monitor and control the power flow through different converters in the system will be presented. The configuration of the hybrid PEV car park emulator is shown in figure 9.1. This car park has the following components: a bidirectional AC-DC inverter together with a RL filter and transformer which connect the car park to the utility grid or the AC subsystem of the hybrid system. A PV emulator which acts as a renewable energy resource is connected to the DC bus. Five lithium battery banks were used to emulate five PEVs parking in the car park, and they are connected to the DC bus through their own bidirectional DC-DC converters, which can be viewed as the chargers for those PEVs. Loads are connected to both the DC side and the AC side. A central aggregator monitors and controls the whole system wirelessly. It collects all the information of the hybrid PEV car park emulator such as DC

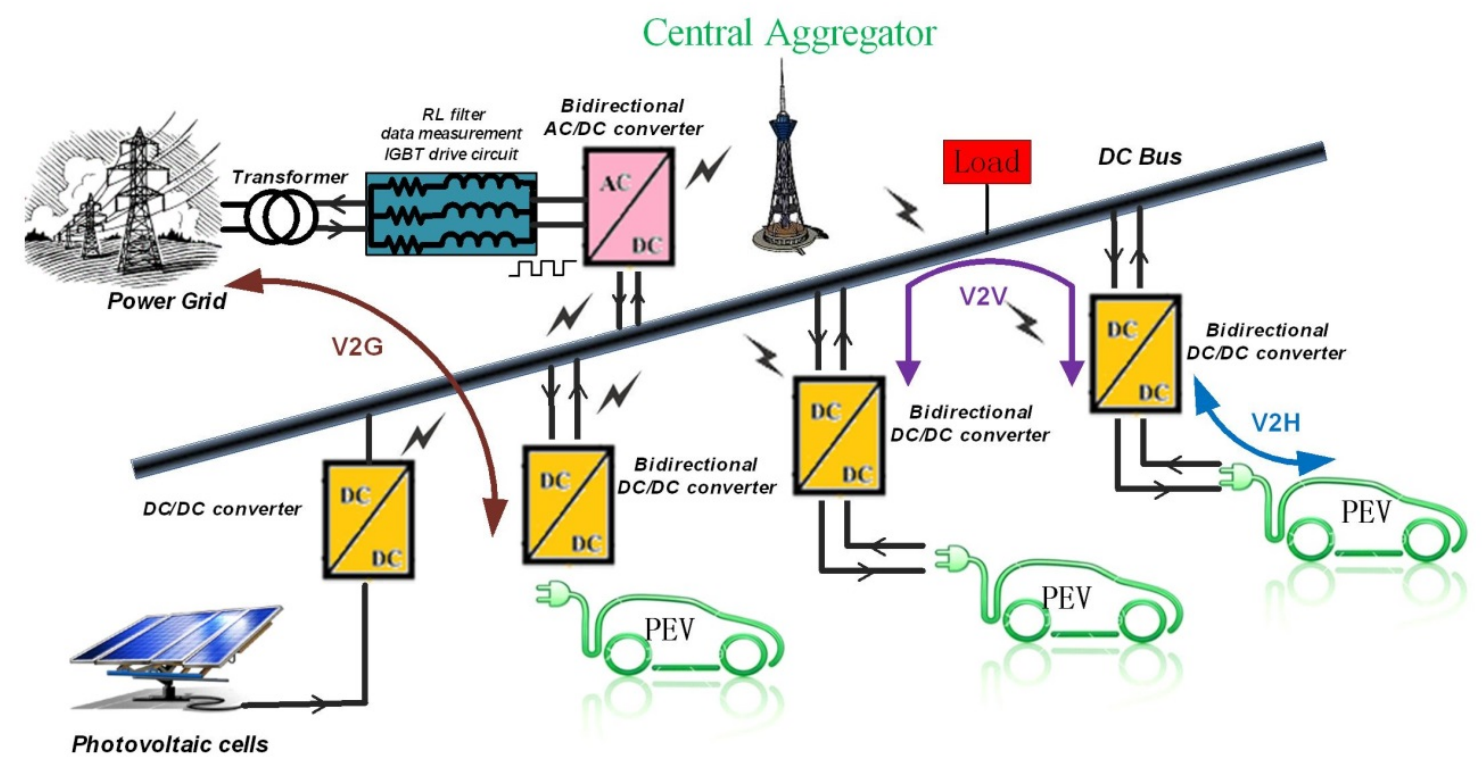

Figure 9.1 PEVs hybrid power system emulator with remote communication and control 
bus voltage, AC bus operating frequency, PEVs' state of charges (SOCs) and so on. Based on all the information, the central aggregator will smartly generate the charging rate signals for the five PEVs individually.

Matlab Simulink and TeraTerm Pro [162] were used in conjunction with the STM-32F Discovery microcontroller and XBEE transceivers [163]. Matlab Simulink is used to design and embed code using the Waijung library block set [164]. This library of components allows the designer to communicate with the microcontroller to build designs into it once the Matlab Simulink model is completed. Instead of directly connecting the microcontroller to the computer, XBEE Series 1 transceivers are used to transmit data to the board. This is done with the software X-CTU which allows the designer to modify all the parameters of each transceiver module to allow for a mesh communication between devices. These modules are then directly connected to the microcontrollers to complete the transfer of data from the user to the microcontrollers.

This methodology is presented as a hands-on engineering solution to the need for a PEV car park that serves to charge and discharge the battery of each vehicle through a bidirectional DC-DC buck-boost converter while it is parked. This car park can be directly connected to the AC utility grid through a bidirectional AC-DC converter to not only charge vehicles, but to serve as an energy storage device to improve the stability of the utility grid by supplying additional power during high demand.

The chapter is organized as follows; the application of low-cost microcontrollers in a PEV car park emulator hybrid power system for power flow control and energy management is detailed in section 9.2. Section 9.3 presents the application of wireless communication control in a PEV car park emulator hybrid power system. PEV car park 
emulator hybrid power system operation with wireless communication and control is given in section 9.4. The performance of the PEV car park emulator hybrid power system and its analysis is presented in section 9.5. Finally, a conclusion is given in section 9.6.

\subsection{Application of low-cost microcontrollers in a PEV car park emulator hybrid power system for power flow control and energy management}

Microcontrollers such as digital signal processors (DSP), field programmable gate arrays (FPGA), programmable logic controllers (PLC), and ARM processors are widely utilized in many engineering fields, especially the fields of modern power system control and management. This is due to their ability to perform complex mathematical calculations with high speed. Also, those digital controllers are flexible, as the implementation involves only software instructions, which is independent of the power electronics devices' sizes.

To make the PEV car park hybrid power system stable on both the AC and DC sides, the most important factor is to keep the keep the power balance between the generators and load on both sides. Therefore, microcontrollers are needed to monitor the hybrid power system states such as the batteries' SOCs, the DC bus voltage, the AC side frequency, and more, while at the same time controlling the power flow through each power electronics device to keep the system stable.

However, most of the microcontrollers such as the DSP processors need to be programmed with special programming languages such as $\mathrm{C}$ or $\mathrm{C}++$. This may complicate the design process and delay the whole design period. To have the ability to use those microcontrollers in power systems, the designers need to be proficient in those 
programming languages. What's more, the program sometimes doesn't directly reflect the control algorithms and the relationship between the inputs and the outputs. Therefore, this may make the design difficult to read and modify. For power system engineers especially, who need to design a system with several power electronics devices, Matlab Simulink is a popular tool to make the simulation. The programming in Simulink is made by a combination of several mathematical blocks, and therefore the logic is very clear. So if we can directly compile and download the tested Matlab Simulink control program to the microcontroller and apply it to the real time hardware experiment, then the design and verification process will be greatly accelerated.

\subsection{1. dSpace controller for bidirectional AC-DC inverter control}

The dSpace hardware together with the virtual test bed software provides an integrated approach that solves most of the power electronics control problems by offering the designer a fully-integrated environment.

The main features of this environment are[165]:

1. Different languages can be used to describe different parts of the system. In particular, Simulink block diagrams can be used to define the control structures;

2. If necessary, proprietary information can be secured;

3. The system simulation can be executed even if not all of the models are available;

4. Controller code can be generated automatically.

In the PEV car park hybrid power system hardware platform design, the dSpace CP1104 is used to control the power flow through the bidirectional AC-DC inverter. The 


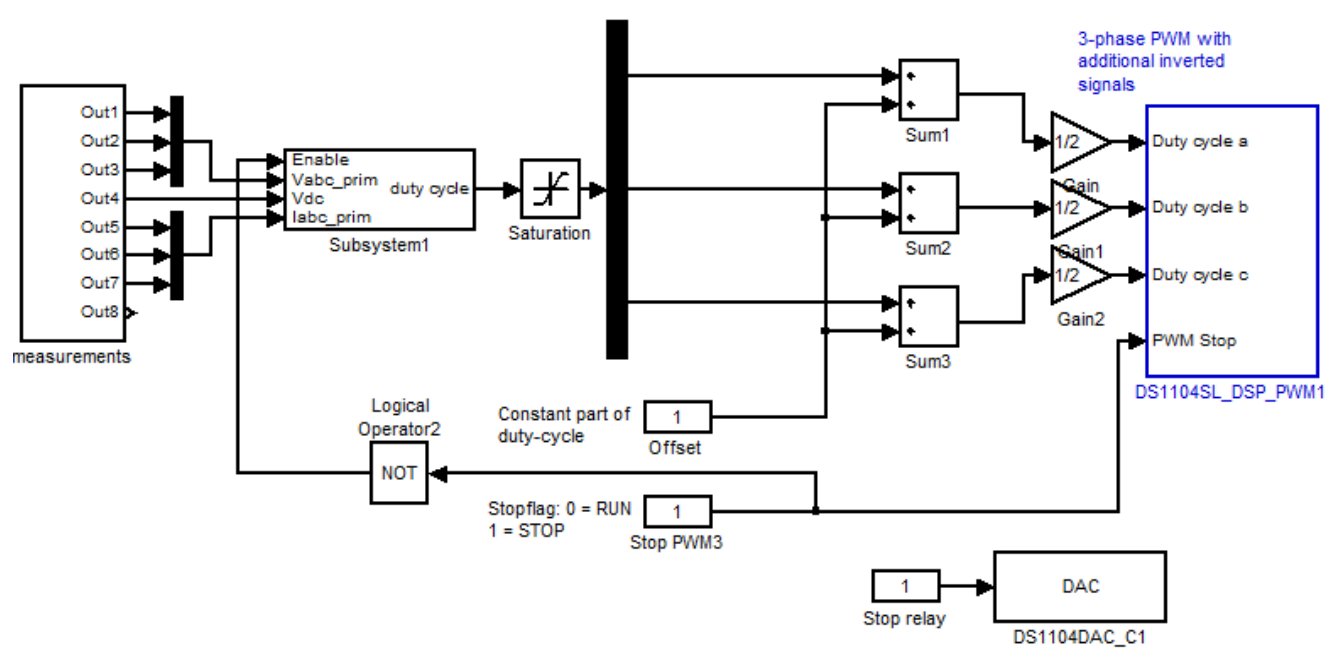

Figure 9.2 Matlab Simulink bidirectional power flow control program

overall Matlab Simulink bidirectional power flow control program is shown in figure. 9.2 .

The voltage and current measurement circuit gives the measured three phase AC bus voltage $u_{a b c}^{g}(t)$, the current flows through the bidirectional AC-DC inverter $i_{a b c}(t)$, and the DC bus voltage $V_{d c}(t)$ to the dSpace ADC ports, the data acquisition Matlab block is shown in figure 9.3.
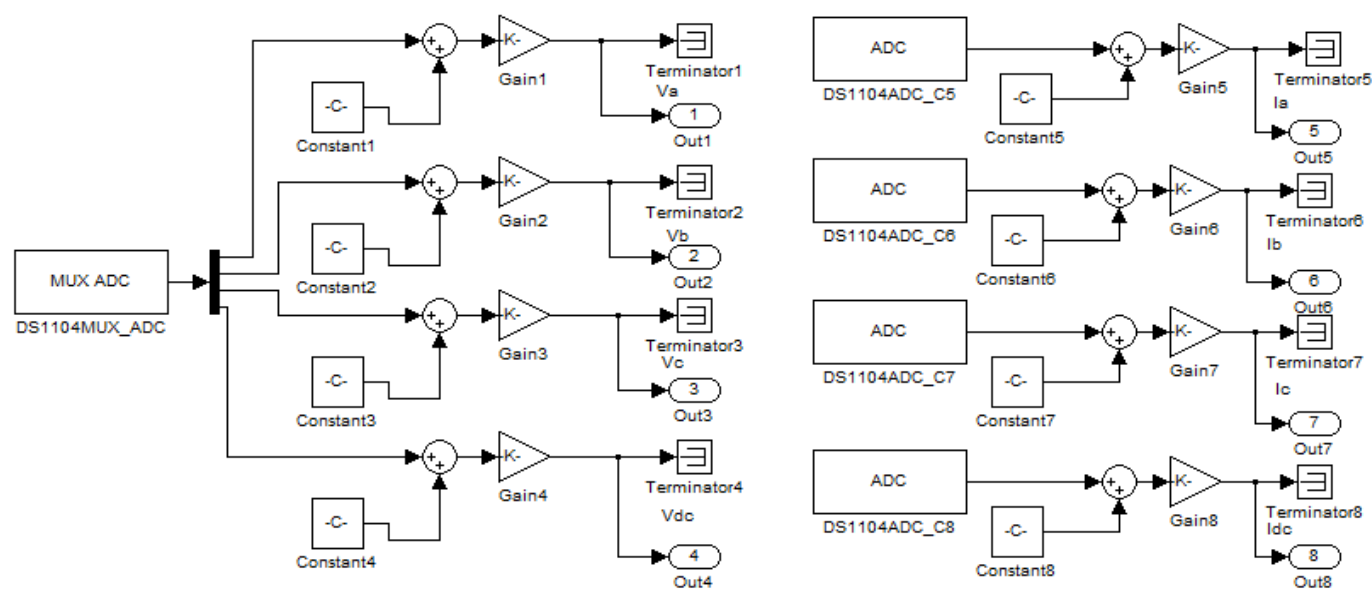

Figure 9.3 Three phase bidirectional AC-DC inverter data acquisition Matlab block 


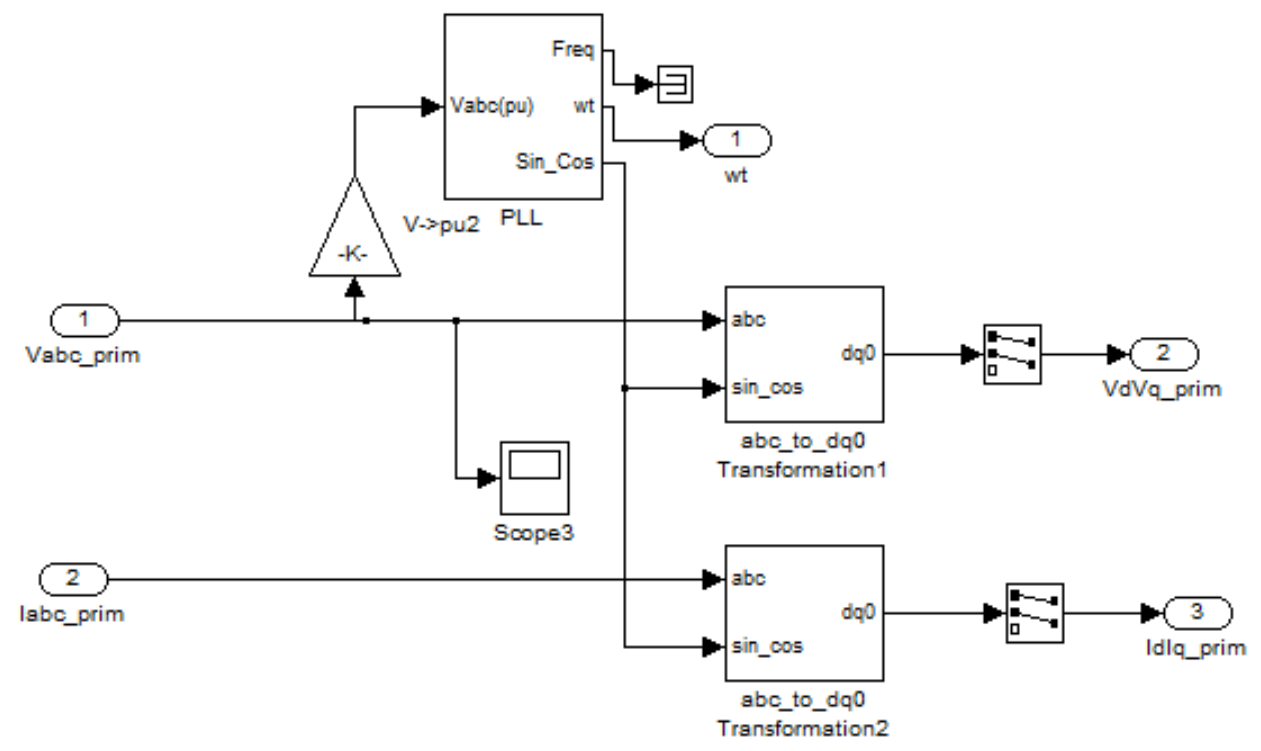

Figure 9.4 PLL and d-q transformation block.

The measured three phase voltage and current signals are sent to the (phaselocked loop) PLL and $d-q$ transform block, which generates the phase angle signals $w t$, $i_{d} i_{q}$, and $V_{d} V_{q}$. This block is shown in figure 9.4.

With the measured $i_{d,} i_{q}, V_{d,}, V_{q}$ and the reference $i_{d}^{f}, i_{q}^{f}$, based on equations (8.24) and (8.25) in Chapter 8, the bidirectional AC-DC inverter output voltage reference signals $v_{d}^{r}(t)$ and $v_{q}^{r}(t)$ are calculated by the current regulator block. The voltage regulator block is shown in figure 9.5 .

The calculated $d-q$ form voltage reference signals $v_{d}^{r}(t)$ and $v_{q}^{r}(t)$ together with the DC bus voltage and phase angle are sent to the $V_{a b c \_r e f}$ generation block, which will generate the normalized $V_{a b c}$ signals, which will be sent to the dSpace pulse width modulation (PWM) block to generate six PWM signals to drive the IGBTs in the bidirectional AC-DC inverter. The $V_{a b c \_r e f}$ generation block is shown in figure 9.6. 


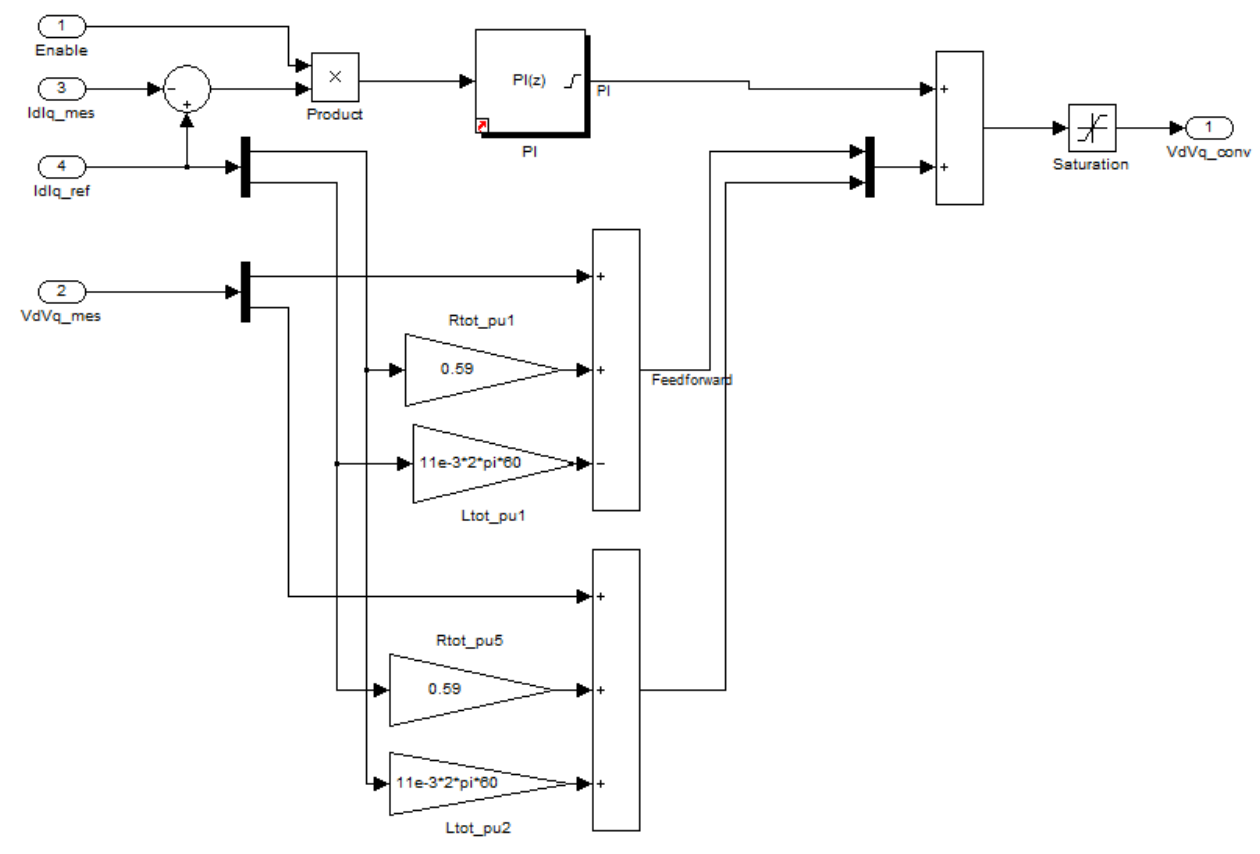

Figure 9.5 Three phase AC-DC inverter active and reactive power decouple control block.

In order to investigate the performance of the developed system, an experimental setup for the bidirectional AC-DC inverter power flow control was constructed. The control program using Matlab Simulink was designed and downloaded to the dSpace CP1104. Figure 8.14 in Chapter 8 shows the photo of the experimental test setup implemented in the laboratory for the developed bidirectional AC-DC inverter. The performance of the proposed system is shown in Chapter 8, Section 8.4.

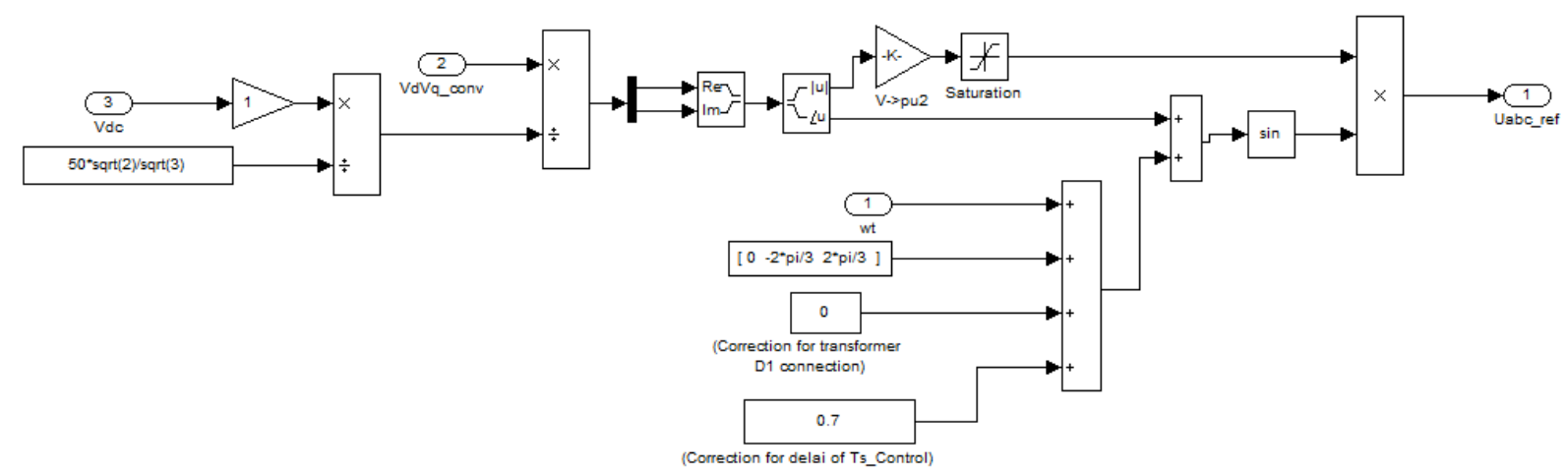

Figure 9.6 Three phase bidirectional AC-DC inverter IGBT duty cycle generation block. 


\subsubsection{STM32F4DISCOVERY controller for bidirectional DC-DC converter control}

Most of the microcontrollers with high performance are very expensive. This may be one of the obstacles that hinder smart grid development. An example of this is the dSpace CP1104 hardware and software which can be used to control projects through Matlab as mentioned in section 9.2.1. The key setback of the dSpace hardware and software is the cost, which is over $\$ 5000$ per unit. This makes it difficult for real industrial applications. Also, by using dSpace as the control interface, the project or system must be constantly connected to the dSpace interface board as well as the computer where the program was created. In addition, dSpace controllers have multiple components which occupy large space in the hardware system. Furthermore, it has a limited processing speed capability. Therefore, except for academic purposes, this hardware is not as popular as other microcontrollers.

In the PEV car park emulator hybrid power system design, there are five battery banks that are used to emulate five individual PEVs charging/discharging behaviors. Therefore, five bidirectional DC-DC converters need to be controlled. Using dSpace CP1104 is a solution, but the cost will be very high since each bidirectional DC-DC converter needs one controller. Also, to monitor five of them, multiple computers with virtual test bed software are needed, which makes it almost impossible for lab scale fulfillment.

Therefore, in this study, STM32F4 DISCOVERY microcontrollers are used to regulate the power flow through the bidirectional DC-DC inverter in the PEV car park emulator. There are five battery banks and five bidirectional DC-DC converters that are 
used to emulate five individual PEVs. Five of these microcontrollers are used to control the power flow through each bidirectional DC-DC converter. Based on the SOC of each PEV, unique charging/discharging signals are sent from a central aggregator to the STM32F4 DISCOVERY through wireless communication by using XBEE wireless transmitters. In this way, V2V service and V2G service can be fulfilled. The transmitters can also work in broadcast mode, which will synchronize all five battery banks and make them work together.

The STM32F4 DISCOVERY microcontroller is shown in figure 9.7. It is a lowcost and easy-to-use development kit to quickly evaluate and start development with an STM32F4 high-performance microcontroller. The microcontroller is running at 160 megahertz (MHz) and executes a 210 MIPS processor which allows it to meet the hard real time requirement. The microcontroller board is based on an ARM cortex M4 with

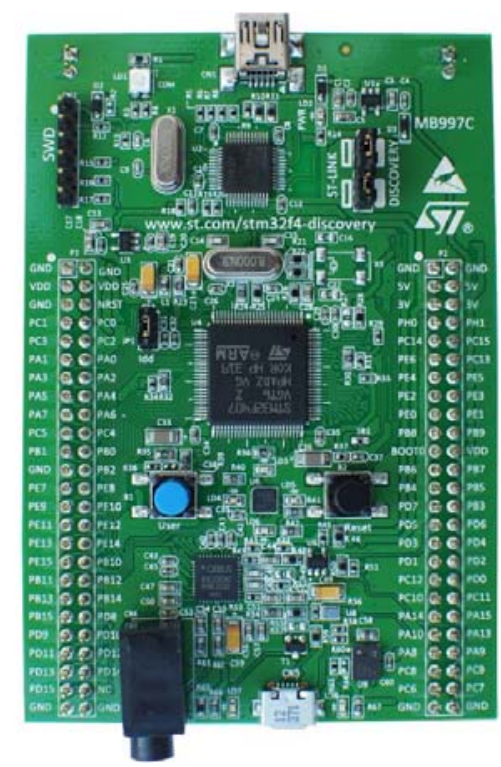

Figure 9.7 STM32F4 DISCOVERY microcontroller 
floating point and DSP extension. The floating point calculation and DSP extension instruction improve the calculation performance which reflects on the total system bandwidth and response time.

The microcontroller support board contains three 12 bit ADCs, each with $2.4 \mathrm{Ms} / \mathrm{s}$ and 8 channel multiplexers with a total of 24 channels. Using multiple ADC modules allows synchronized sampling of voltage and current. By utilizing the high sampling rate and DSP functionality, accurate measurements can be obtained by sampling and filtering signals with digital filters before applying the control algorithms.

For communication the microcontroller contains 6 universal high speed asynchronous serial ports, SPI and I2C. These communication ports were utilized to communicate with wireless modems and exchange data with an embedded web server. An Ethernet interface is also supported. This Ethernet interface can be utilized with a light weight embedded webserver to build a web interface for remote monitoring and control. The firmware was stored in an on board 1 Mbyte protected flash memory while the webserver resources were stored in an external SD card connected via an SPI interface.

For DC-DC converter control, the microcontroller supports up to 6 channels of PWM. This PWM module uses dedicated timers and digital comparators to control the duty cycle which minimize the processor overhead. $186 \mathrm{~KB}$ onboard static memory is used for temporary data storage during runtime.

Since the microcontroller ADC block can only receive a $0 \mathrm{~V}-3.3 \mathrm{~V}$ analog signal, and the bidirectional current control of the DC-DC converter current transducer output 
signal may be a negative value, a signal converting circuit is needed. The signal converting circuit converts the signal from the range of $[-10 \mathrm{~V} 10 \mathrm{~V}]$ to $[0 \mathrm{~V} 3.3 \mathrm{~V}]$, which
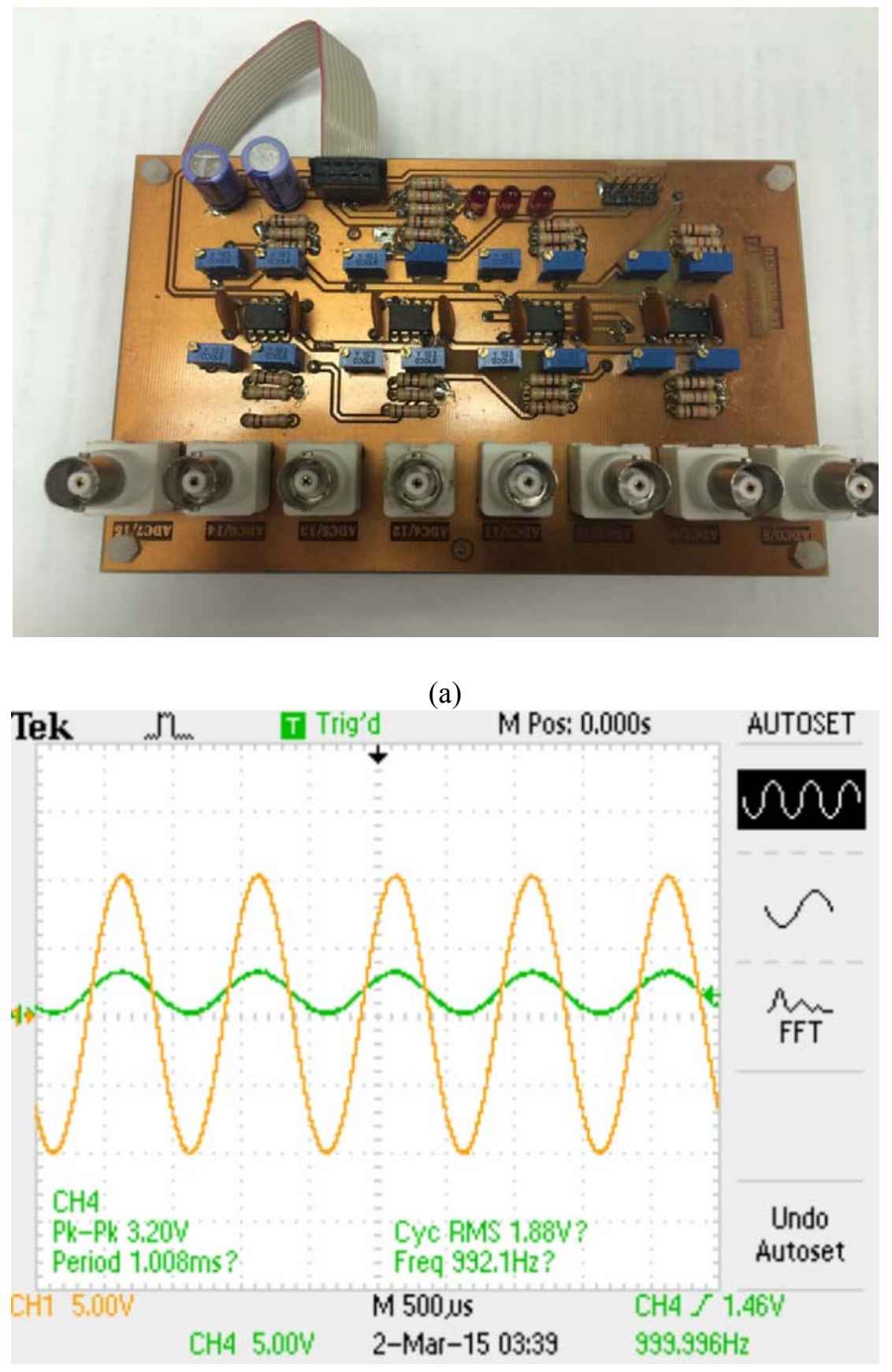

(b)

Figure 9.8 Signal converting circuit for STM32 (a) circuit hardware (b) circuit performance. 
is within the operating range of the STM32F4 microcontroller. The signal converting circuit and its performance are shown in figure 9.8 (a) and (b).

\subsection{Application of wireless communication control in the PEV car park emulator hybrid power system}

ZigBee was used as the wireless communication transmitter and receiver in this design. This is a specialized protocol for small, self-programming mesh network devices based on the IEEE 802.15.4 wireless standard. ZigBee devices are designed for low power consumption and are easy to implement.

The low cost of the ZigBee device can facilitate the commercial deployment of the desired PEV networks at large PEV car parks. The simplicity of the ZigBee allows for inherent configuration. The redundancy of the network also provides low maintenance. Moreover, this device can be operated at $868 \mathrm{MHz}, 915 \mathrm{MHz}$, and even 2.4 gigahertz (GHz) radio bands, which can maintain higher communication compatibility. Also, the 2.5 GHz operation can reach around $250 \mathrm{kbps}$, which can meet the estimated bandwidth requirements for PEV charging control applications. In large PEV car parks, there may be a large number of communication nodes. ZigBee's use of the IEEE 802.15.4 physical layer (PHY) of the Open Systems Interconnection (OSI) model and the link layer device (MAC) allows the network to handle any number of devices. The operation range of ZigBee devices is 400 feet $(120 \mathrm{~m})$, which in most situations is enough for the design of a large scale PEV car park. The main features of the ZigBee are listed below:

- Low power consumption, operated at $3.3 \mathrm{~V}$ with $40 \mathrm{~mA}$

- Fast communication capability, 250kbps Max data rate 
- $2 \mathrm{~mW}$ output $(+3 \mathrm{dBm})$

- Large operation distance $400 \mathrm{ft}(120 \mathrm{~m})$ range

- Built-in antenna

- Six 10-bit ADC input pins

- High security, 128-bit encryption

- Modules operate within the ISM 2.4 GHz frequency band

To use ZigBee transceivers together with the STM32F4 DISCOVERY microcontrollers to monitor and control multiple bidirectional DC-DC converters, appropriate numbers of microcontrollers, transceivers, XBEE adapters, and one XBEE Explorer interface board with a USB cable are needed. The TeraTerm Pro is used to transfer data between transceivers while the X-CTU is used configure the communication

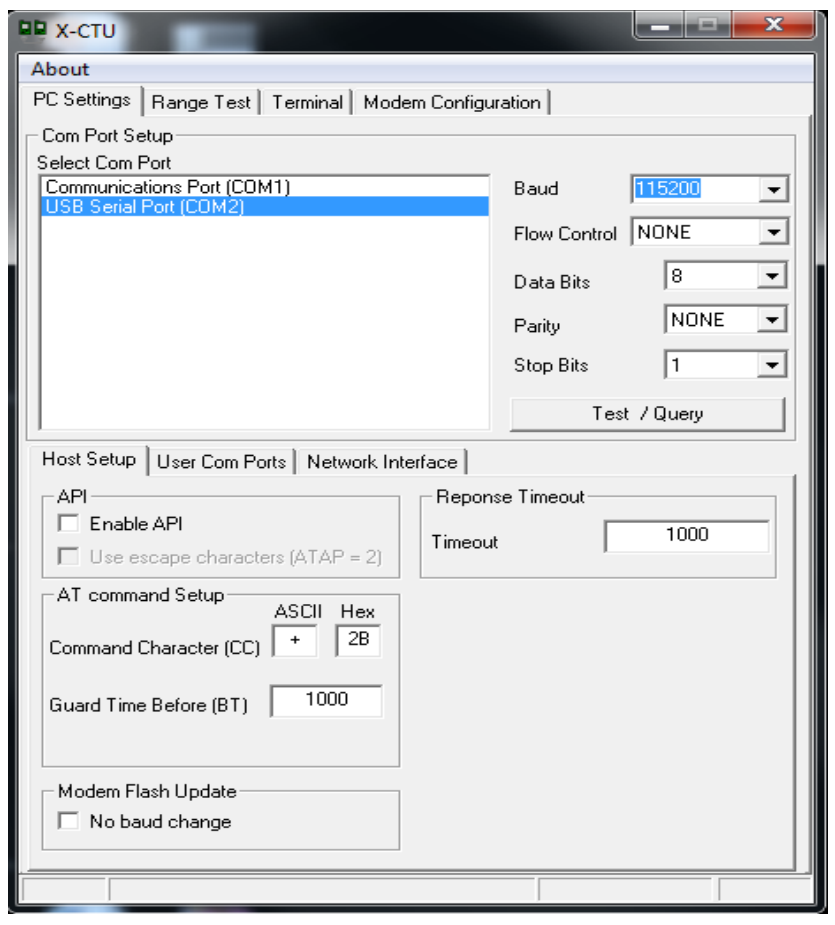

Figure 9.9 X-CTU program interface 
between them. The X-CTU program interface is shown in figure 9.9. To set up the system, the designer begins by opening the program after having plugged a transceiver into the interface board and then connects the board onto the computer via USB. The specific port used will then show up on the ports list. As can be seen, the transceiver is currently connected to COM2. The designer can verify which port is used by the transceiver by going to the device manager from the control panel in the Windows machine and looking at the port designations. The Baud rate refers to the speed data is to be transferred. Flow control manages the rate of data to prevent data overflow or dropped packets of information. The Data Bits, Parity, and Stop Bits are rarely altered and should remain the same for this process. Once these default parameters are verified, click Test/Query. The designer should be presented with a small window that verifies the connection of your XBEE module.

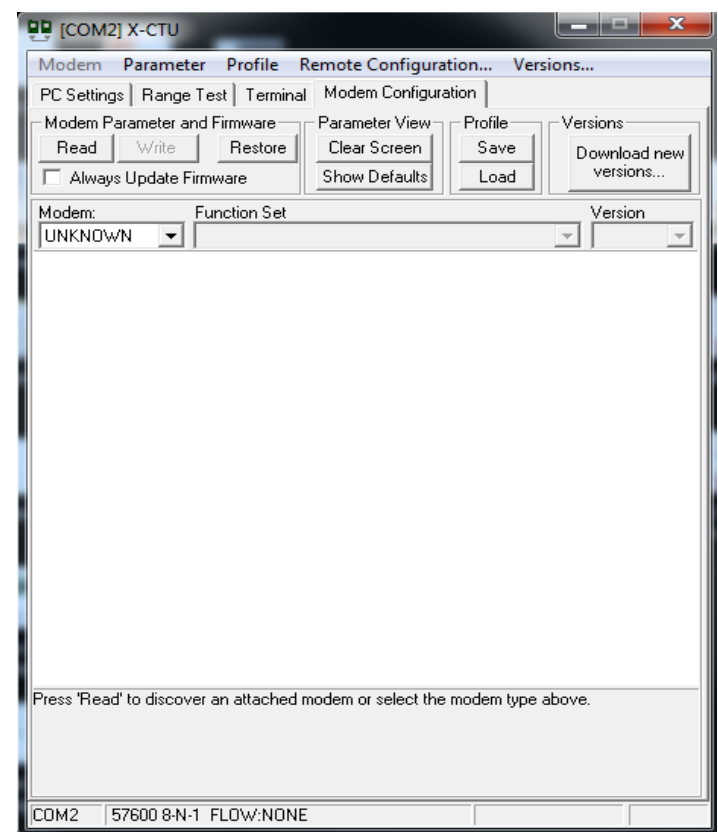

Figure 9.10 X-CTU configuration 
Then, click on the Modem Configuration tab. The designer will be presented with the following image, shown in figure 9.10.

Once on this tab, click Read. After a few seconds the designer should be presented with the following figure 9.11. Here are all the parameters that are on the module. The only ones of importance for the time being are Channel, PAN ID, and Interface Data Rate (Baud Rate) which are usually colored blue. In order to have the proper mesh communication between all of the XBEE modules, these three parameters have to be identical. The Matlab Simulink design and TeraTerm Pro software must also use the same Baud rate. The designer can view all the possibilities of these parameters on



Figure 9.11 X-CTU parameters setting 
the XBEE Series 1 data sheet found online. In general, the designer should use the letter $\mathrm{B}$ or $\mathrm{C}$ for the Channel and then a combination of four numbers for the PAN ID and verify that this specific combination is not being used by any other devices to avoid communicating with their modules. In general, the XBEE modules work best using the Baud Rate 57600. They work on any Baud Rate listed but it is recommended to use them at 57600 . Once the appropriate values for these three parameters have been chosen, click Write. Repeat this process for all of the modules.

Once this step is complete, the designer can move on to configure TeraTerm Pro. TeraTerm Pro is a terminal emulator that emulates a variety of different computer terminals and supports internet communications such as telnet and SSH and also allows serial communications which is what is used in this procedure. At this point, the designer will click serial and choose the same port as the XBEE interface board from before and press OK. Alter the settings to the following, shown in figure 9.12:

Now, click Setup again and go to Serial Port. Verify that all of the parameters are identical to the ones used in the experiment. The designer will be now be presented with a clear console that is ready for input commands that are sent to the microcontroller to

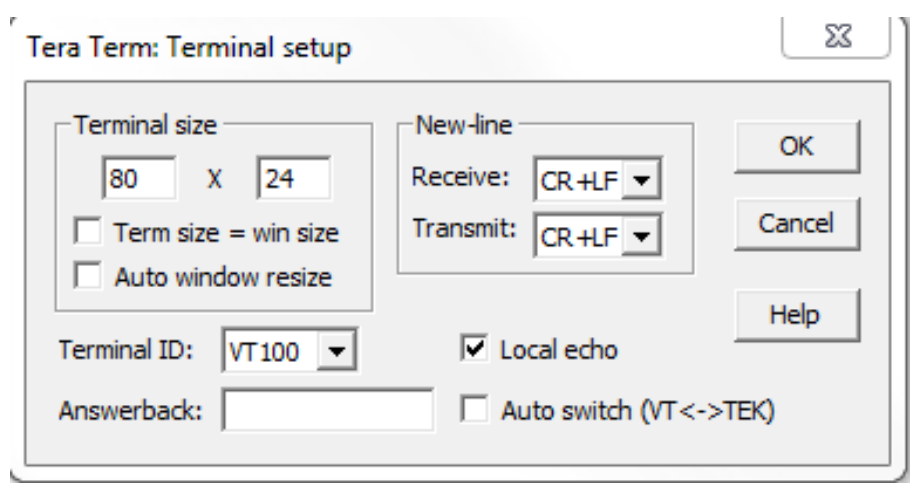

Figure 9.12 TeraTerm Setup 
generate the PWM signal with a user controlled duty cycle.

The next step is to properly connect the transceivers to the microcontrollers using the XBEE Explorer adapters. Place the transceiver on an adapter and using four of the thin spiral colored cables, connect the following pins together;

- Pin GND of the adapter to any GND of the microcontroller

- Pin 5V of the adapter to Pin 5V of the microcontroller

- Pin RX (receive data) of the adapter to Pin B6 of the microcontroller

- Pin TX (transfer data) of the adapter to B7 of the microcontroller

Repeat the process for the appropriate number of microcontrollers the designer wishes to use. In this process, five microcontrollers were used to generate five different PWM signals with variable duty cycles. The designer should then provide power to each microcontroller. This can be done in one of two ways; directly connect the microcontroller to the computer via USB, or connect the other Pin $5 \mathrm{~V}$ to the positive terminal of a power supply and a GND Pin to the negative terminal of the power supply. Before connecting, adjust the power supply to a constant $5 \mathrm{~V}$. To connect multiple microcontrollers to the power supply, use a breadboard to connect the $5 \mathrm{~V}$ pins and GND pins in parallel with the power supply.

\subsection{Energy storage system operation with wireless communication and control}

After establishing the wireless communication, control signals can be sent remotely by a central aggregator to the STM32 microcontrollers to regulate the charging/discharging rates of the battery banks by controlling the current flow through the bidirectional DC-DC converter. Also, the battery banks terminal voltages can be 
measured and sent back to the central aggregator by the STM32 microcontrollers as feedbacks.

\subsubsection{Battery banks SOC monitoring web server system design.}

With the feedback of the battery banks terminal voltages, using the battery model proposed in Chapter 5, the SOC of each battery bank can be estimated. With all the SOCs of the battery banks, the central aggregator can make charging/discharging ratios for each battery bank. Also, with this design, after collecting the SOCs, different power dispatching methods can be tested on this platform.

To design the central aggregator, another STM32 microcontroller was used. It was also equipped with a Zigbee wireless transmitter, which allows it to communicate with other STM32 microcontrollers that are used to control the power flow through the bidirectional DC-DC converters. The central aggregator communication can be operated in Peer-to-Peer (P2P) mode or broadcasting mode. For energy storage system control, P2P is preferred since it can help the system balance the SOCs of different battery banks.

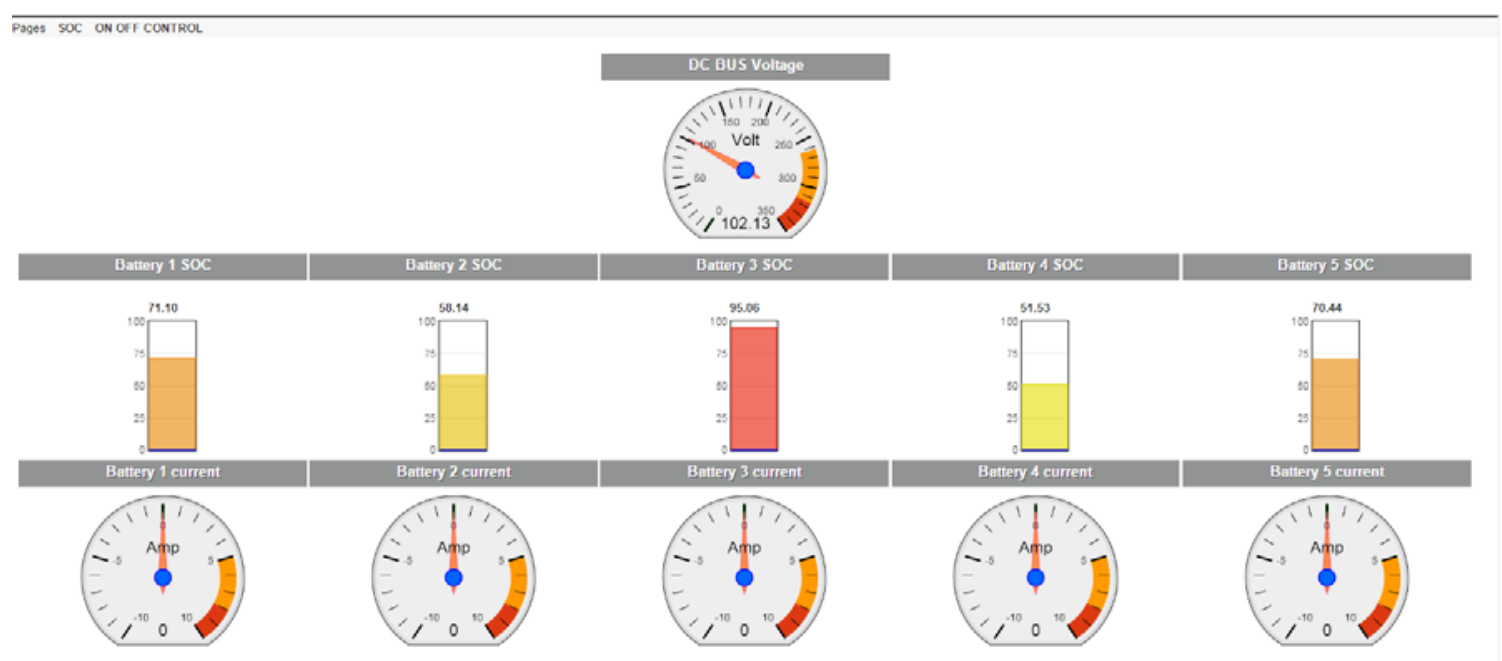

Figure 9.13 Battery banks SOC monitoring web server interface. 
The STM32 microcontroller used as central aggregator in this study was connected to the local network through the Ethernet interface. It can act as a server to monitor the whole energy storage system. The battery banks SOC monitoring web server system is shown in figure 9.13. This design can be used as the central aggregator for future PEV networks in a car park. It can also collect information such as the power requirement by the PEVs, next period output power from renewable energy sources, utility grid conditions, and energy prices to make a smart charging solution for effective dispatch of the energy to different PEVs in the network with low charging cost and low impacts while fulfilling some $\mathrm{V} 2 \mathrm{G}$ and $\mathrm{V} 2 \mathrm{~V}$ services.

\subsubsection{Bidirectional DC-DC converter power flow remote control}

To remotely control the bidirectional DC-DC converter, the STM32
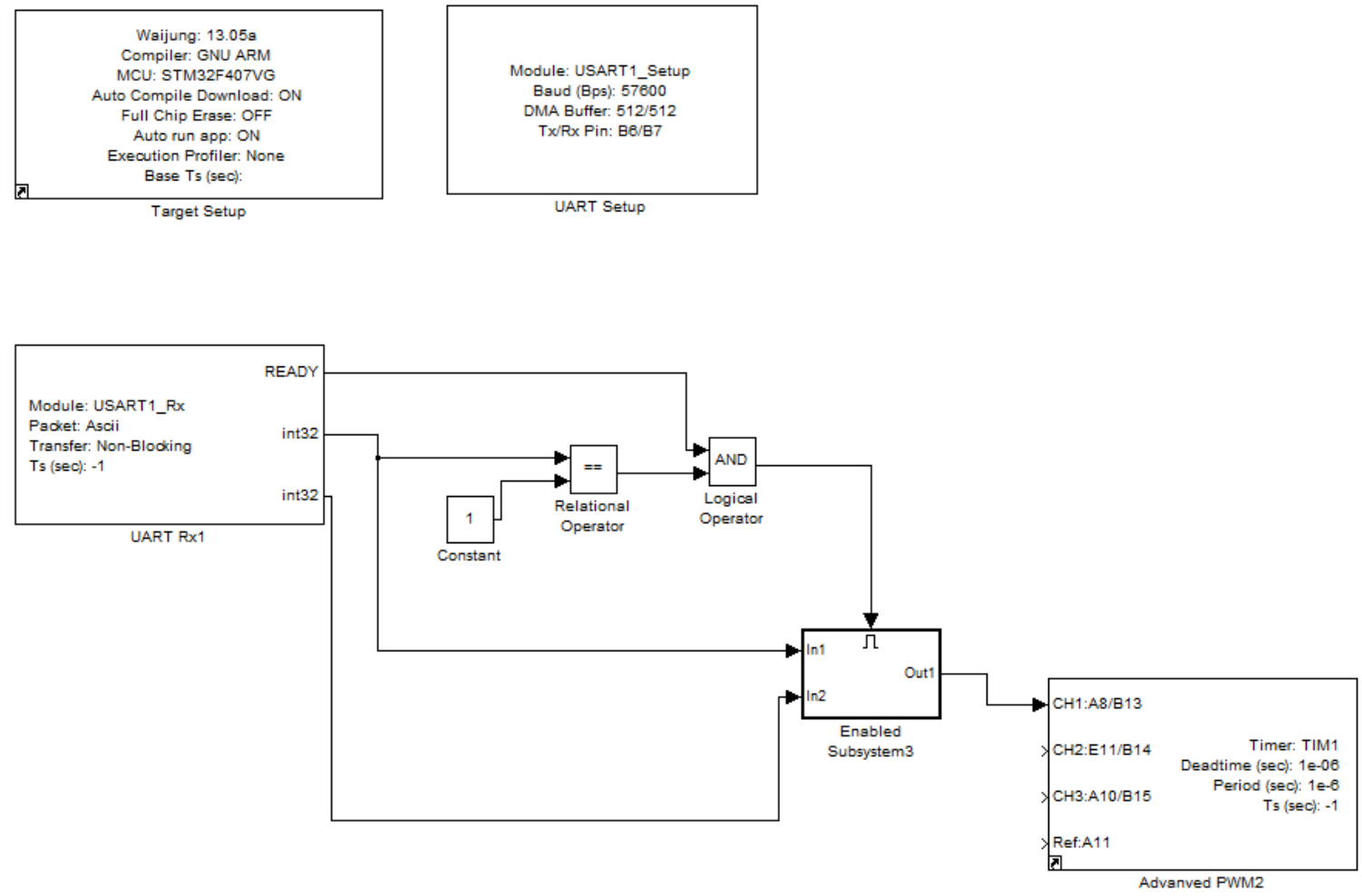

Figure 9.14 Bidirectional DC-DC converter PWM control program 
microcontroller needs to receive the control signal from the central aggregator. Different kinds of control signals can be used, such as the duty cycles that can be directly given to the PWM block to generate the PWM signals to drive the IGBTs in the converter, current reference signals that can be used to directly regulate the current flow in the converter, or droop coefficients that can be used to help the DC subsystem regulate the DC bus voltage. The following example shows the case that duty cycle is used to remote control the power flow.

At this point, all that is left is the Matlab Simulink design using the Waijung library block sets. The design built into each board that generates multiple PWM signals with variable duty cycles is shown in figure 9.14.

All of these blocks are found in the library browser under Waijung Block sets. The Target Setup block is added to all designs that utilize the STM-32F microcontrollers and allows for the design to be properly built into the board. There are no settings that

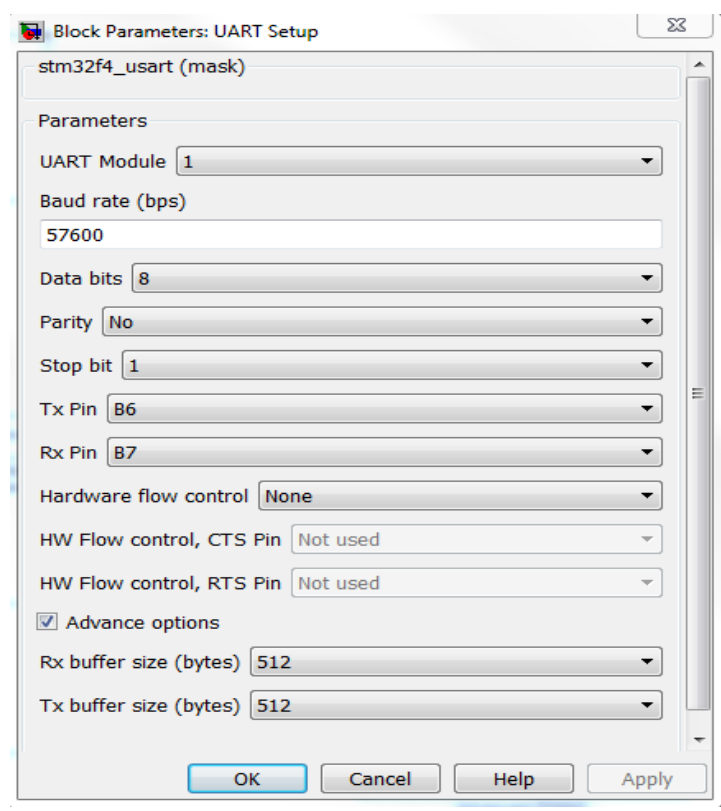

Figure 9.15 Zigbee parameters for STM32F microcontroller. 
need to be altered from the default block. The UART Setup block is where the communication parameters are altered. This block is also where the pins used for communication are located on the microcontroller. Figure 9.15 shows the parameters for Zigbee connecting:

The UART Rx1 block is the block that allows the designer to communicate directly with the microcontroller of choice and its respective duty cycle. Figure 9.16 shows its parameters. As can be seen, the UART module is the same as before. The transfer of data is chosen as Non-Blocking. With this option, new data can be entered without blocking any procedure currently being carried out. This allows the central aggregator to change duty cycles in real time when other duty cycles are present. The packets of data are sent as ASCII characters. The line 'add \%d duty \%d' is what the designer inputs into the TeraTerm Pro terminal. Each board has a specific address. Since this design has five microcontrollers, there are five different options. The following

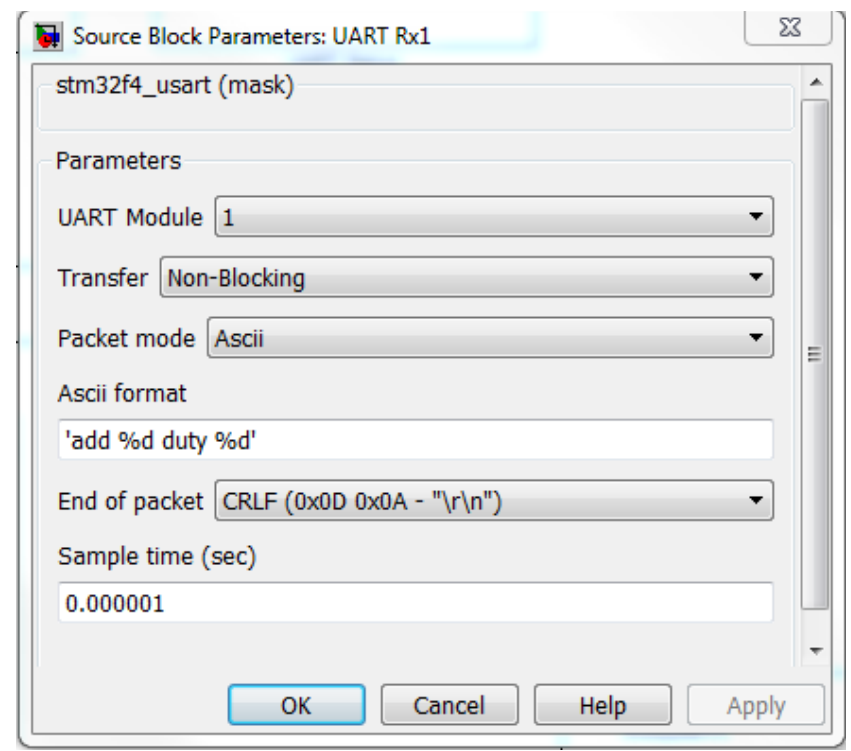

Figure 9.16 UART Rx1 block parameters. 
example illustrates how the line is used:

'add 1 duty 56 '

In this line, the central aggregator wants board 1 to generate a PWM signal with a duty cycle of 56 percent.

In this design, the address is sent to a relational operator that compares it to an integer. This integer refers to the address of a specific board, with each board having the exact same design built into it with the exception of this constant. Board 1 will have the number 1 in this box. Board 2 will have the number 2 in this box, etc. If the user typed the same number for the address as the constant, then the AND gate will register as 1 and the Subsystem will be enabled, allowing the duty cycle to pass through to the board. That address number along with the duty cycle is sent into the subsystem where the output is then sent to the Advanced PWM block. The output of this PWM is located on Pin A8 to GND. The PWM generation subsystem is shown in figure.9.17.

In figure 9.17 it is observed that the address (In1) and duty cycle (In2) enter the

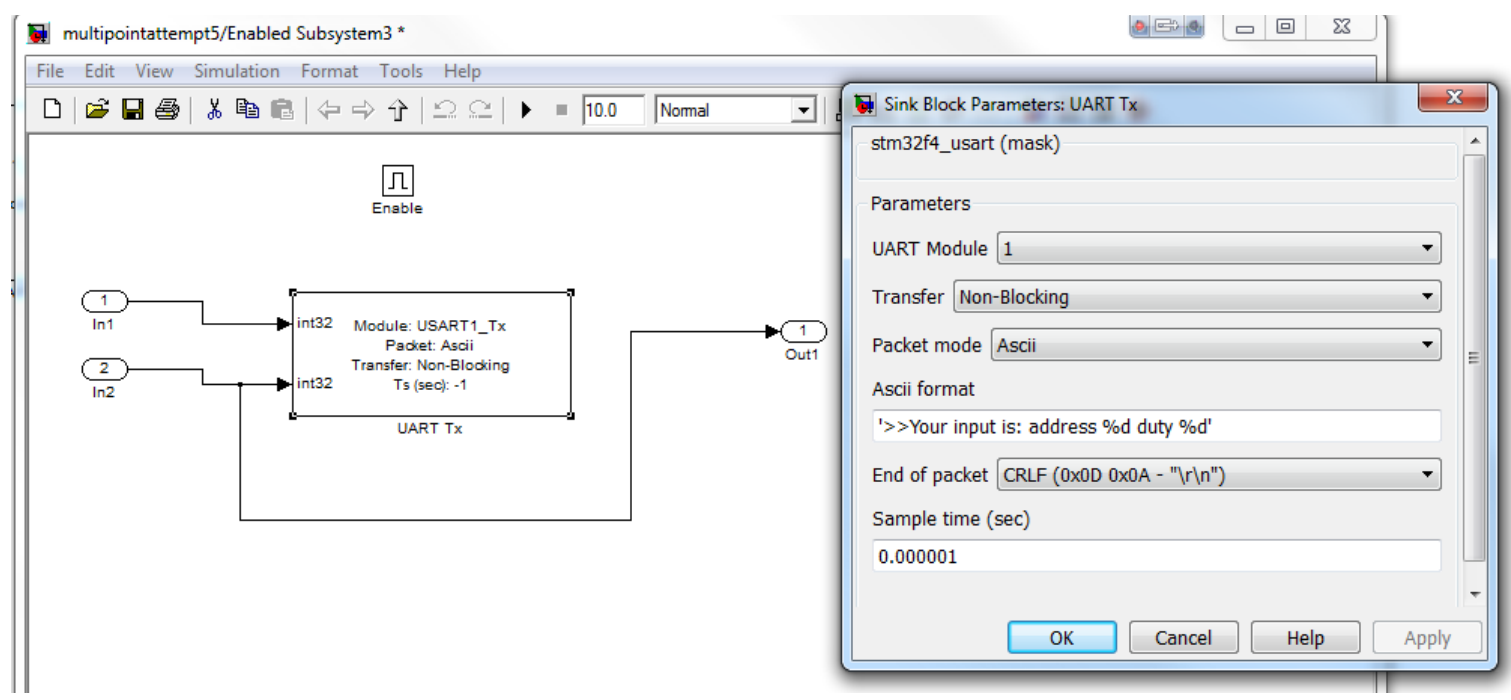

Figure 9.17 Bidirectional DC-DC converter PWM generation block. 


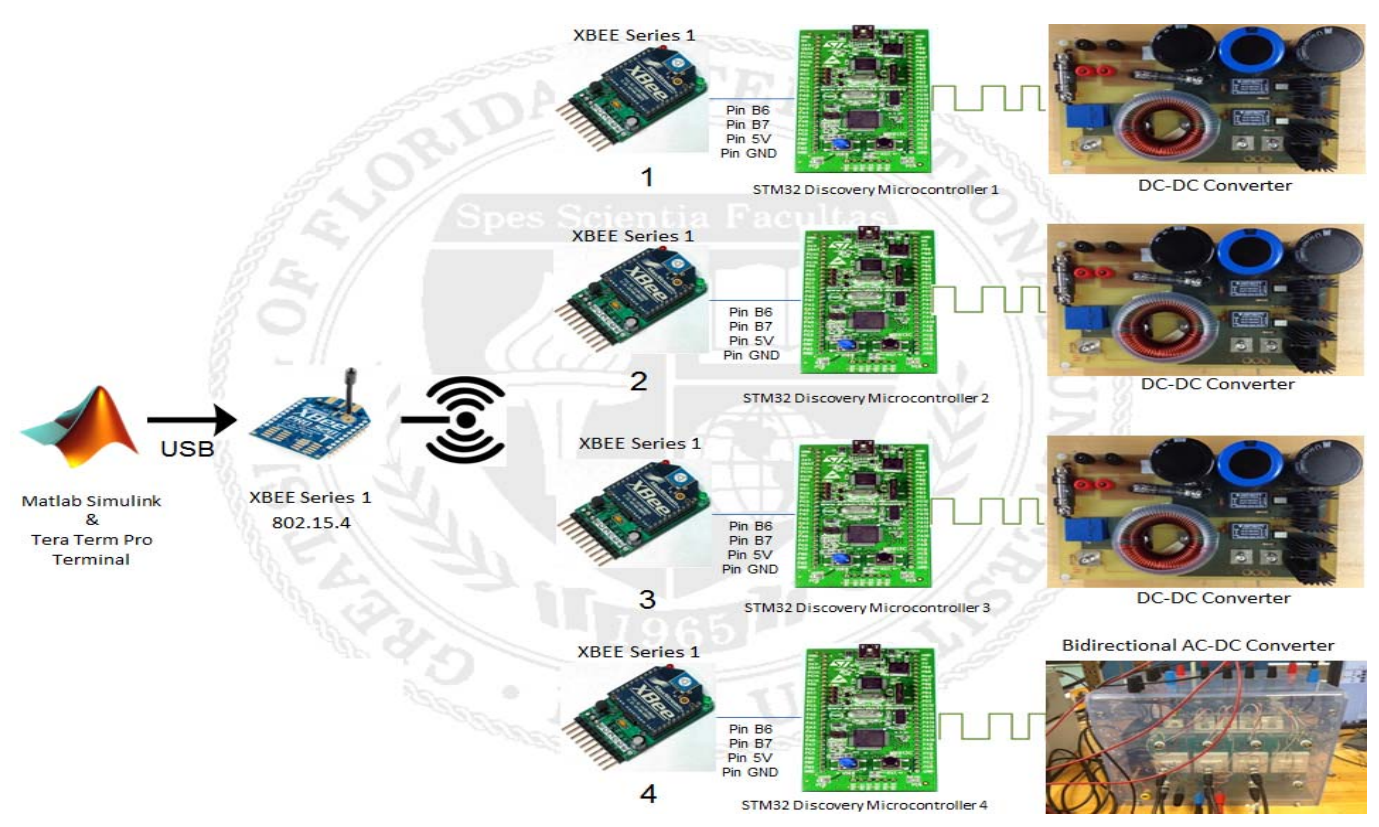

Figure 9.18 Battery banks monitor and control system.

transfer module so that the designer receives a message in the terminal of what he/she inputted. In the above example, the output would be:

>>Your input is: address 1 duty 56

Also, observe that the duty cycle $(\ln 2)$ is then outputted from the subsystem to Out1. This output is what reaches the PWM block. Board 1 gets the build with 1 as the constant. Board 2 gets the build with 2 as the constant, etc. The overall design should resemble the following components shown in figure 9.18:

In this design, the PWMs are used to control the switching frequencies of the IGBT's in converters to control power flow. Once all boards have been compiled appropriately, connect as above. Now, the central aggregator is ready to actually use the TeraTerm Pro program to control the duty cycles of multiple PWMs simultaneously. Figure 9.19 shows an image of the terminal being used to control five PWM signals: 


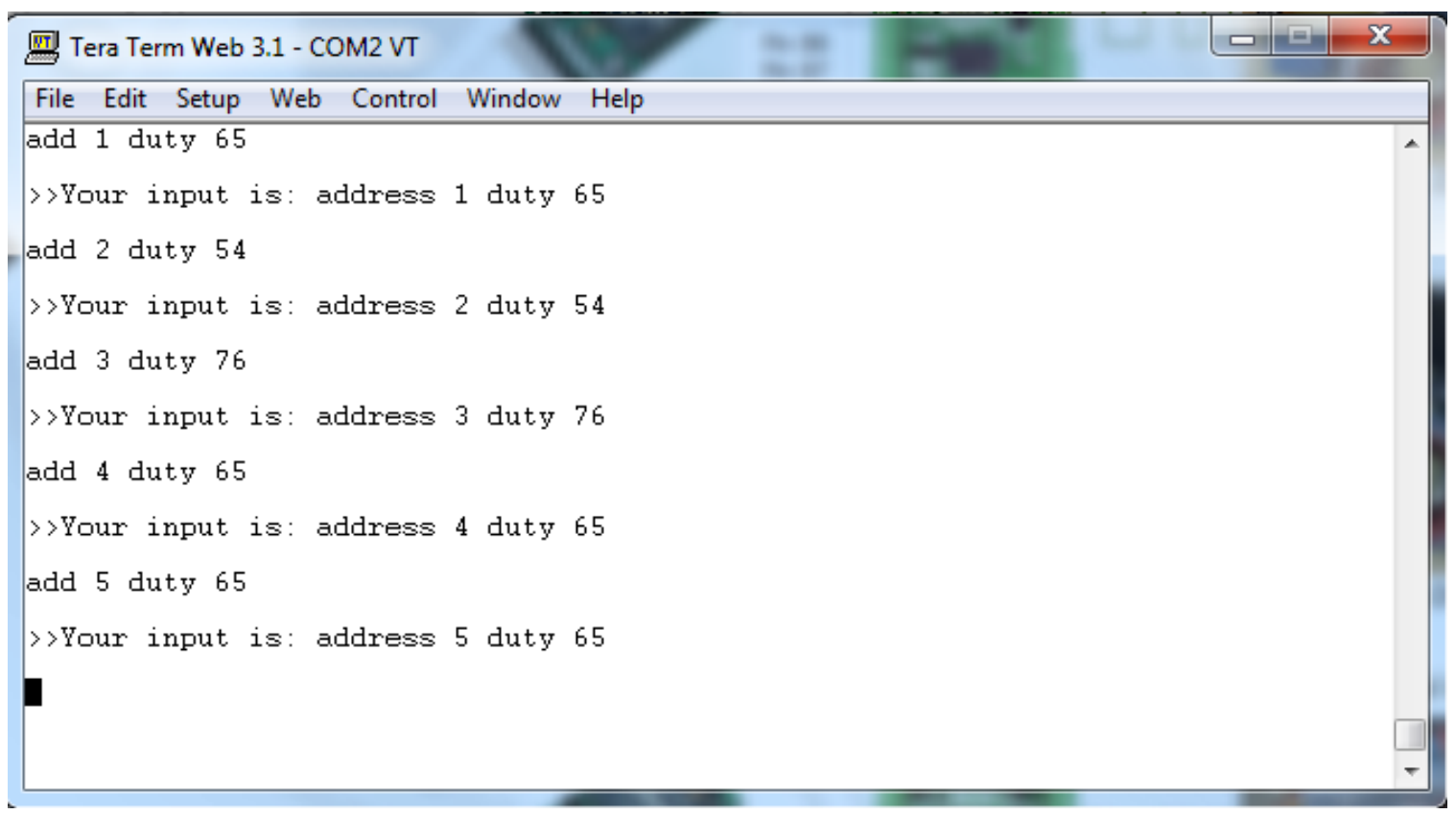

Figure 9.19 TeraTerm User Terminal

\subsection{Conclusion}

This chapter describes the methodology of using transceivers and microcontrollers to remotely monitor and control the power flow of the energy storage systems in a hybrid AC-DC power system. The descriptions of how to utilize dSpace CP1004 and STM32F microcontrollers to control the power flow through bidirectional AC-DC inverters and DC-DC converters are given in detail. Also, using the STM32F to design a web server that acts as a central aggregator to monitor and control the power flow of five battery banks is presented. The proposed method gives a hardware solution to design the remote smart charging/discharging control for PEV networks in a car park. 
10. Laboratory Scaled Plug-in Electric Vehicles Car Park Infrastructure Emulator Design

\subsection{Introduction}

In this chapter, the design and implementation of a laboratory-based hybrid ACDC power system is presented. This hybrid power system is implemented at the Energy Systems Research Laboratory at Florida International University, to emulate a plug-in electric vehicle (PEV) car park infrastructure. It consists of an AC subsystem and a DC subsystem. The AC subsystem has a grid connection point that can connect the hybrid power system to the university utility grid. The AC subsystem also has two AC generators with $13.8 \mathrm{KVA}$ rated output power, which can be operated in slack mode and power-voltage mode. On the DC side, a $6 \mathrm{~kW}$ photovoltaic $(\mathrm{PV})$ emulator is connected to the DC bus to act as a renewable energy source. Five lithium-ion battery banks with 51.8 rated terminal voltage and 21 Ah capacity are used to emulate the behavior of five PEVs in the car park.

The values of the parameters of the power electronic devices utilized, in addition to the various filters and microcontrollers in the system, are given. Moreover, the control techniques and the controllers designed to operate the developed hybrid AC-DC power system are discussed. Several experimental results were included to verify the validity of the developed PEV car park emulator and its applicability as an educational and research tool to study modern design, operation, and control aspects related to hybrid AC-DC 
power systems, as well as renewable energy and PEV integration into hybrid AC-DC power systems.

In addition, a smart energy management system for the developed PEV car park emulator was designed. The energy management system has a central aggregator that can remotely monitor the state of charge (SOC) of the PEVs in the car park and smartly distribute the available energy to PEVs with different SOCs. Moreover, a coordination control scheme among the $\mathrm{AC}$ subsystem and $\mathrm{DC}$ subsystem is designed with vehicle to grid (V2G), vehicle to house (V2H), and vehicle to vehicle (V2V) services. The results

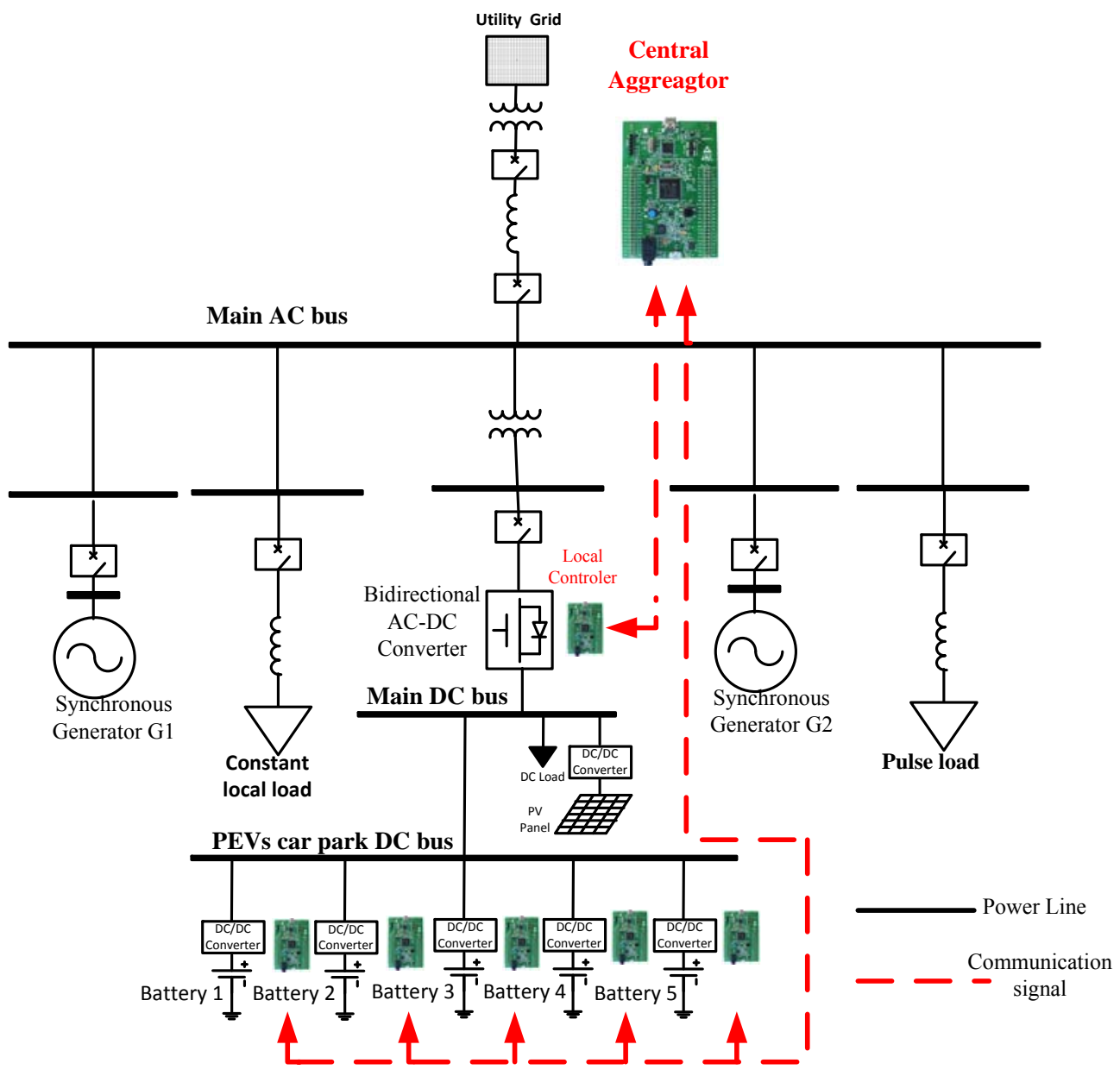

Figure 10.1 Laboratory based PEV car park emulator hybrid power system infrastructure 
obtained verify that the proposed hybrid AC-DC power system power management methodology can be an effective solution for future power systems with high penetration of renewable energy sources and PEVs.

This chapter is organized as follows: the PEV car park infrastructure emulator overview including the grid connected inverter, battery banks, bidirectional DC-DC converter, and wireless communication scheme are described in Section 10.2. Section 10.3 presents the application of a real time energy management algorithm to car park emulator. The hybrid AC-DC power system with PEV car park performance analysis with V2G verification is given in Section 10.4. A conclusion of this chapter is given in Section 10.5.

\subsection{PEV car park infrastructure emulator overview}

The schematic diagram of the developed laboratory based PEV car park emulator hybrid power system infrastructure is shown in figure 10.1. The system consists of a DC micro grid and an $\mathrm{AC}$ micro grid. In the $\mathrm{AC}$ micro grid, the two generators can be operated as a slack generator or a power-voltage controlled generator, depending on if the system is operating in islanding mode or grid-connected mode. There are constant loads and pulse loads in the AC micro grid, which may influence the system frequency and voltage. The DC micro grid has a PV emulator, and a DC-DC boost converter that is used to inject the power from it to the DC bus. The DC bus is also connected with five lithiumion battery banks through five bidirectional DC-DC converters. The SOCs of the battery banks are monitored by five individual STM32 microcontrollers. The microcontrollers send the SOCs of the battery banks to a central aggregator, which monitors the state of 
the whole system and makes charging/discharging control signals to those battery banks to keep the system stable.

\subsubsection{Grid connected inverter and AC filter}

The circuit diagram of the grid connected inverter and $\mathrm{AC}$ filter in the proposed hybrid AC-DC power system is shown in figure 10.2. The active and reactive power decoupled control technology proposed in Chapter 8 is applied to control this bidirectional AC-DC inverter. When the system is operated in grid-connected mode, the inverter is used only to control the DC bus voltage by regulating the $I_{d}$, which makes the DC micro grid act as a pure resistance load with unity power factor. When the hybrid power system is operated in islanding mode and there is a slack generator on the AC subsystem, the bidirectional AC-DC inverter is operated in current control mode. Based on the battery banks SOCs, the central aggregator sends power transfer signals to the bidirectional AC-DC inverter to regulate the power flow in between the AC and DC micro grids. When there is no slack generator on the AC micro grid, or the slack generator is disconnected from the AC micro grid in fault conditions, the bidirectional

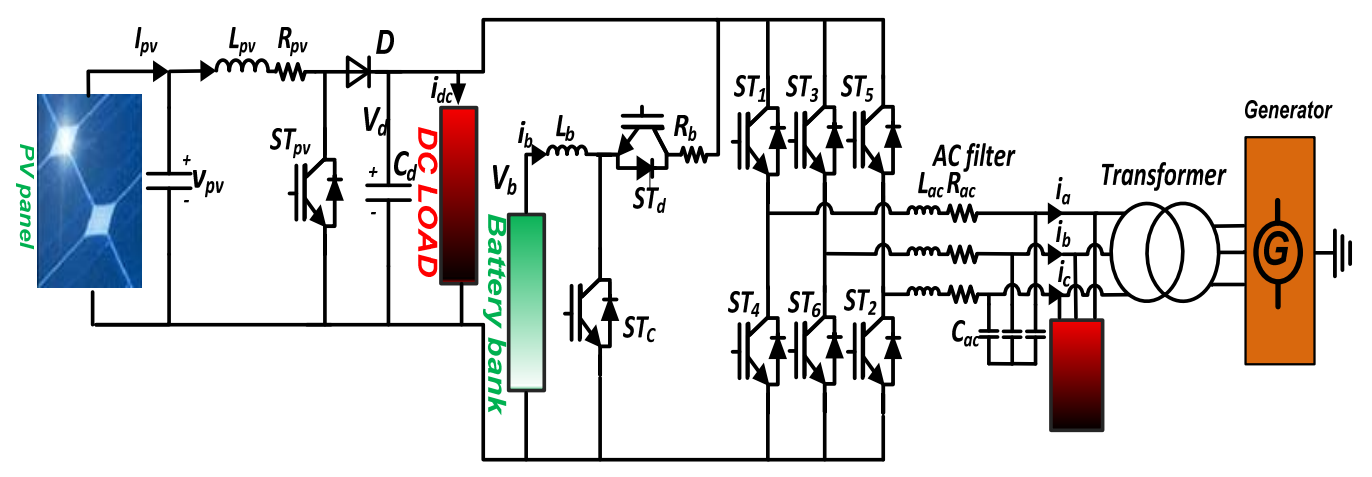

Figure 10.2 Circuit diagram of the grid connected bidirectional AC-DC inverter with filter in the proposed hybrid power system. 




Figure 10.3 Lithium-ion battery module

$\mathrm{AC}-\mathrm{DC}$ inverter will automatically transfer to frequency regulation mode to help the AC micro grid regulate the system frequency and voltage amplitude.

\subsubsection{Battery banks}

Five lithium-ion battery banks are connected to the same DC bus on the DC micro grid. The lithium-ion battery bank module is shown in figure 10.3. Each single battery bank consists of 14 lithium-ion battery cells connected in serial, with $51.8 \mathrm{~V}$ terminal voltage and $21 \mathrm{Ah}$ capacity. Those five battery banks are used to emulate five individual PEVs in a car park. The battery banks SOCs are monitored by measuring the terminal voltages. The lithium-ion battery model proposed in Chapter 5 is applied to estimate the SOCs of the battery banks. The DC PEV car park micro grid is shown in figure 10.4.

\subsubsection{Bidirectional DC-DC converter}

Five of the bidirectional DC-DC converters proposed in Chapter 8 Section 8.3 were used to control the charging/discharging of the five lithium-ion battery banks. Those converters can be viewed as PEV chargers in the future power system. 


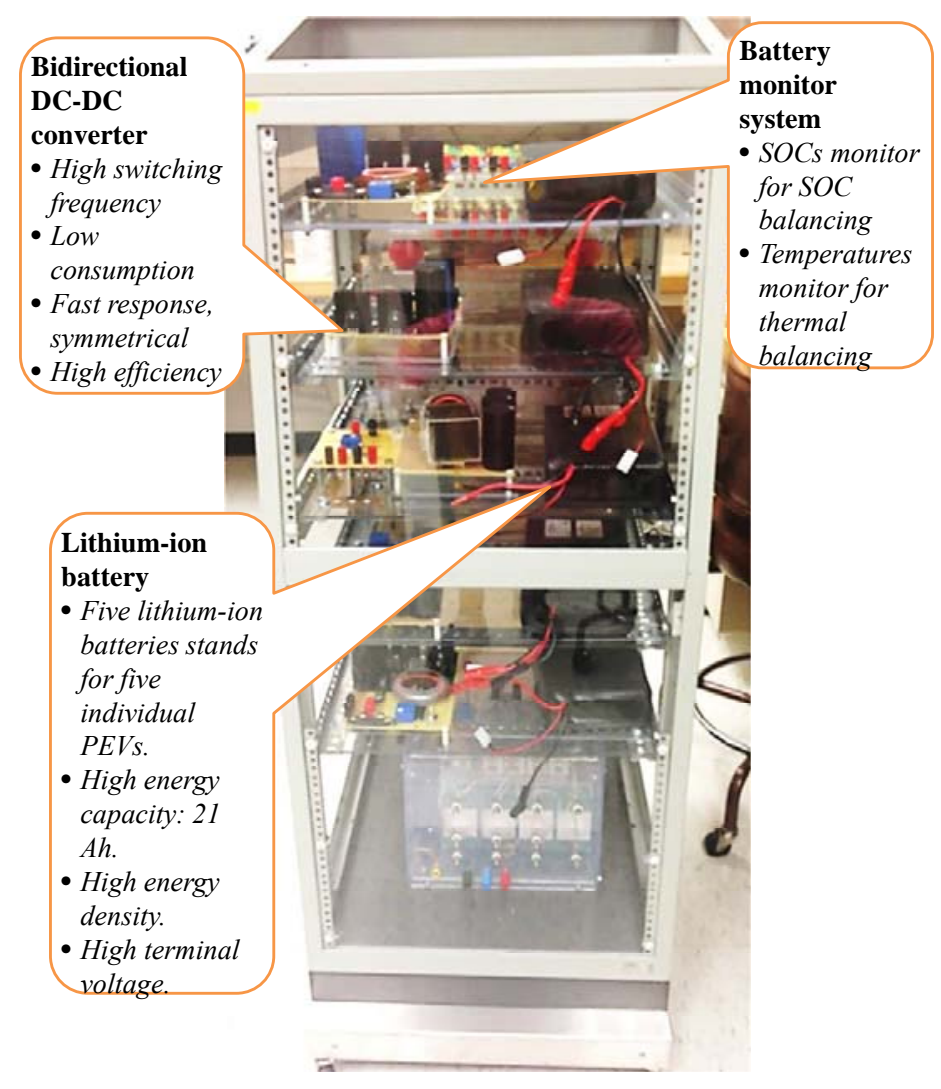

Figure 10.4 PEV car park emulator DC micro grid.

When the system is operated in the grid connected mode, the five converters are operated in current controlled mode and regulate the charging/discharging rate of the battery banks based on the reference charging/discharging signals received from the central aggregator. When the whole system is disconnected from the utility grid, the bidirectional DC-DC converters will change to voltage control mode, to regulate the DC bus voltage. Five STM32F microcontrollers were utilized to control the power flow of those five bidirectional DC-DC inverters individually. With those functions, the bidirectional DC-DC converter can use the energy stored in the battery banks to regulate the DC bus voltage. Furthermore, with the cooperation of the bidirectional AC-DC inverter, the battery banks can help the system regulate the $\mathrm{AC}$ micro grid frequency and 
voltage amplitude. Those can be viewed as the rudiment of future V2H and V2G services from the PEV network.

\subsubsection{Wireless communication and control}

The methodology proposed in Chapter 9 for utilization of Zigbee wireless communicators and STM32 microcontrollers to remotely monitor DC micro grid states and control the charging/discharging of battery banks in the hybrid power system is also applied to the PEV car park hybrid AC-DC power system. This concept makes the system operate in a distributed manner, and can be utilized in future power systems to coordinate several distributed components (such as several PEVs distributed in different places of a city) and control them to fulfill some services together. Furthermore, with the established central aggregator, different PEV smart charging algorithms can be tested on this platform. The new control algorithms can be easily tested by downloading the program to the central aggregator, and the rest of the PEV car park emulator won't be influenced, which will accelerate future PEV smart charging research.

\subsection{Application of a real time energy management algorithm to the car park emulator}

The PEV real time smart energy management algorithm is embedded in a STM32 microcontroller used as the central aggregator. The central aggregator can communicate with the other five STM32 microcontrollers which are used to control the current flow through the bidirectional DC-DC converters. The Zigbee wireless transmitters are used for remote communication. The communication is also bidirectional. These five STM32 microcontrollers measure the terminal voltage of the battery banks and send it to the 
central aggregator. The aggregator collects all five battery banks' terminal voltages and estimates their SOCs. A priority rank is calculated based on the SOCs of the battery banks. Battery banks with higher SOC will be assigned a lower rank. With the rank of the battery banks, different smart charging algorithms can be designed and tested on the central aggregator. Based on the algorithm, the central aggregator will generate five charging/discharging rate signals and send them back to these five STM32 microcontrollers individually. After receiving the charging/discharging reference signals, these five microcontrollers will adjust the current flow through the converters controlled by them.

An example of the control program for applying the adaptive droop control of multiple battery banks charging management proposed in Chapter 5 Section 5.6 to the central aggregator is shown in figure 10.5. After gaining the rank of the battery banks, the central aggregator will monitor the state of the DC bus voltage. If the measured voltage is lower than the reference voltage, set as $100 \mathrm{~V}$ in this experiment, all of the battery banks need to inject energy to the DC bus. If the measured voltage is higher than the DC bus

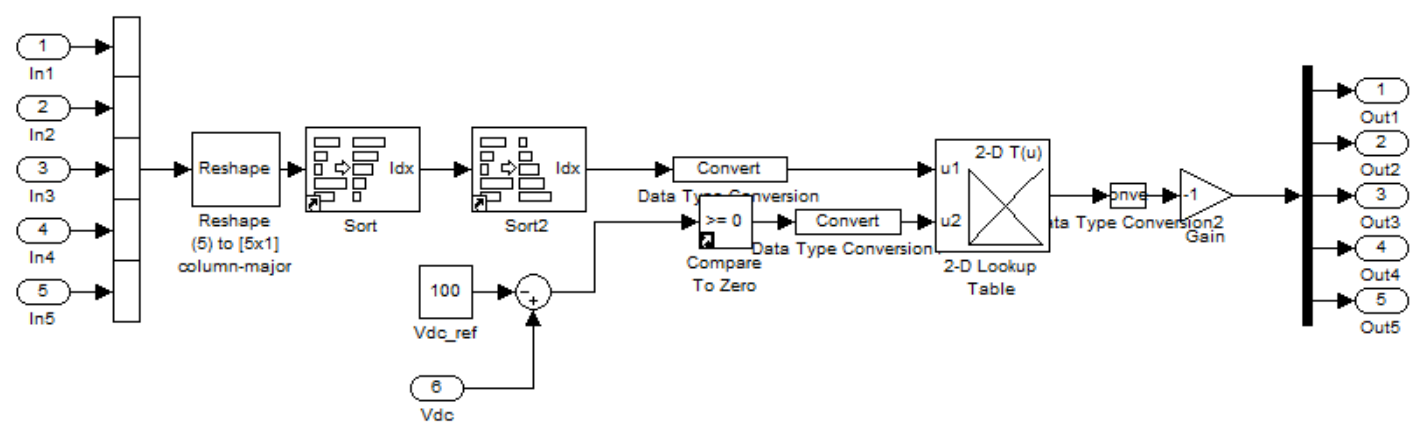

Figure 10.5 Adaptive droop control program for multiple battery banks charging management 
voltage, all of the battery banks need to absorb energy from the DC bus. After that, a 2-D lookup table is used to generate the droop coefficients for five individual battery bank controllers. Those droop coefficients will be sent to the STM32 microcontrollers by the central aggregator through the wireless communicator. After receiving the droop coefficients, the microcontroller will generate a charging/discharging reference signal and regulate the current flow through the bidirectional DC-DC converter it controls. The flowchart is shown in figure 10.6.

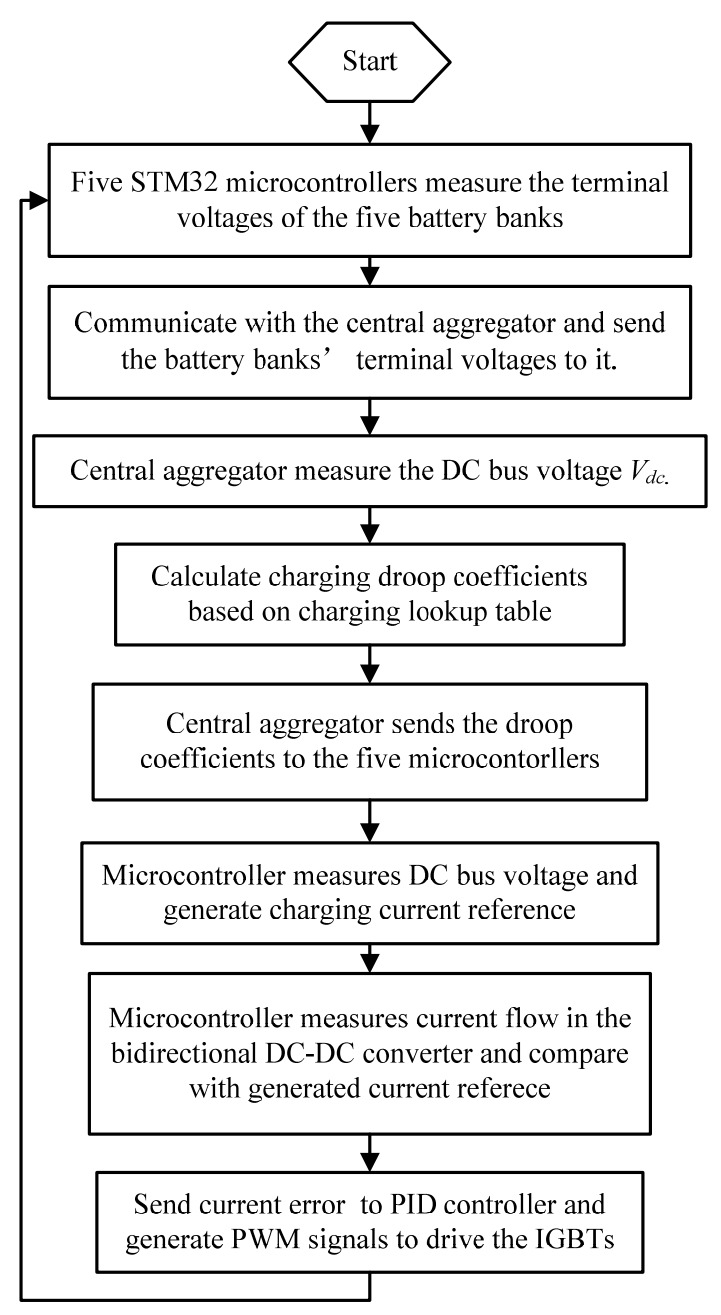

Figure 10.6 Flowchart of the battery banks smart energy management control process 


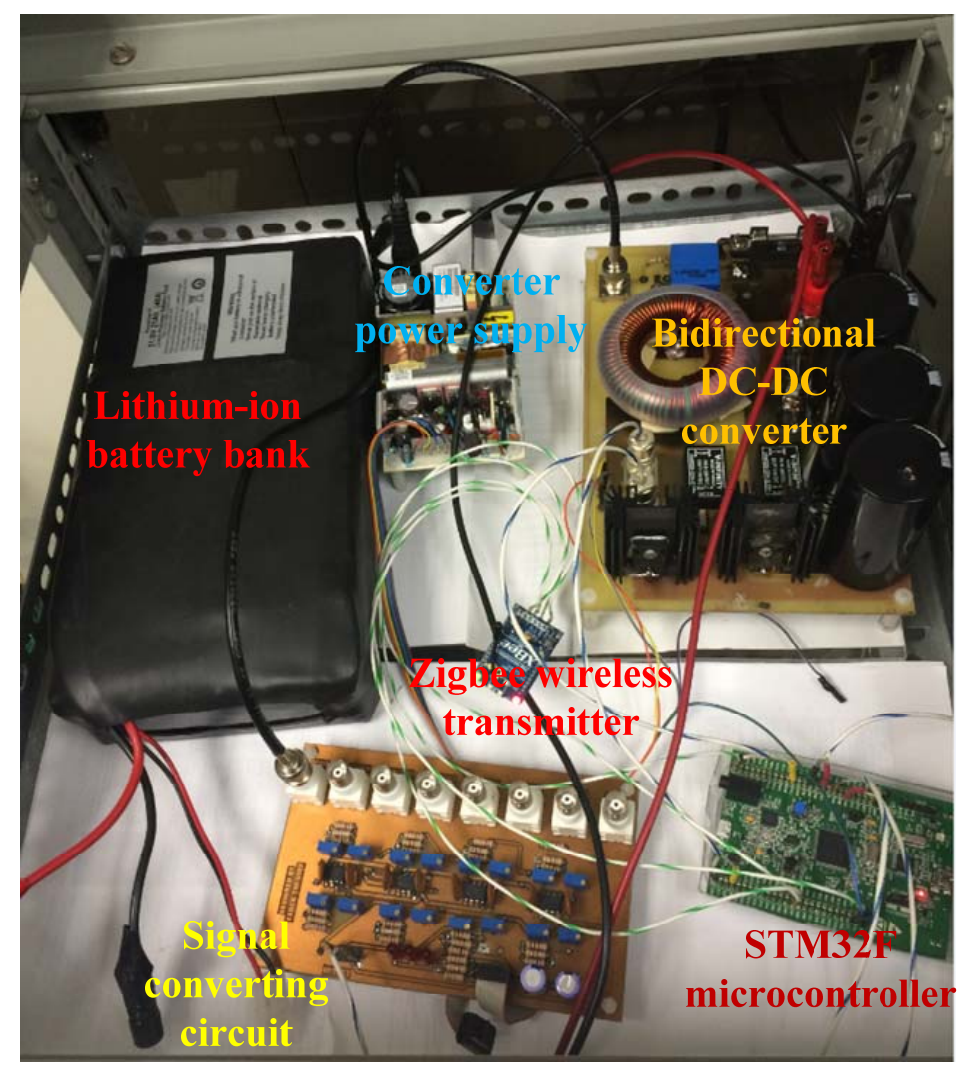

Figure 10.7 Hardware configuration of one PEV emulator

The PEV car park emulator has six floors. The top five floors emulate five PEVs with their DC-DC converters. The last floor contains a DC power supply which acts as a PV emulator. The hardware components on one floor are shown in figure 10.7.

\subsection{Hybrid AC-DC power system with PEV car park performance analysis}

To further verify the proposed control algorithm for hybrid AC-DC power system operation with pulse load mitigation, a hardware experiment was done. For security and protection reasons, the hardware platform was scaled down. The system hardware configuration is shown in figure 10.8. It has two induction generators G1 and G2. G1 is connected at bus 1 and operated as a slack generator to regulate the AC subsystem 
frequency. Generator G2 is connected at bus 2 and operated in power-voltage control mode, which gives a constant output power. The AC subsystem also has a $750 \mathrm{~W}$ constant load connected at bus 3 and a $600 \mathrm{~W}$ pulse load connected at bus 4 with a 1 second period. The DC micro grid is connected at bus 4 through the bidirectional AC-DC inverter.

Two scenarios were tested: AC subsystem frequency regulation and pulse load support from DC subsystem. In the first scenario, the DC micro-grid has a frequency regulation function, but in regular situations this function is disabled to prevent it from fighting with the slack generator. However, as mentioned in Chapter 8 Section 8.2.3, the control algorithm should have the ability to distinguish the frequency drop caused by a slack generator disconnection from the frequency variation caused by a pulse load. The load, output power from two generators, and power from the DC micro grid is shown in figure 10.9 (a). The system frequency response is shown in figure 10.9 (b). Before the slack bus disconnection, the DC subsystem keeps injecting a constant 100W active power

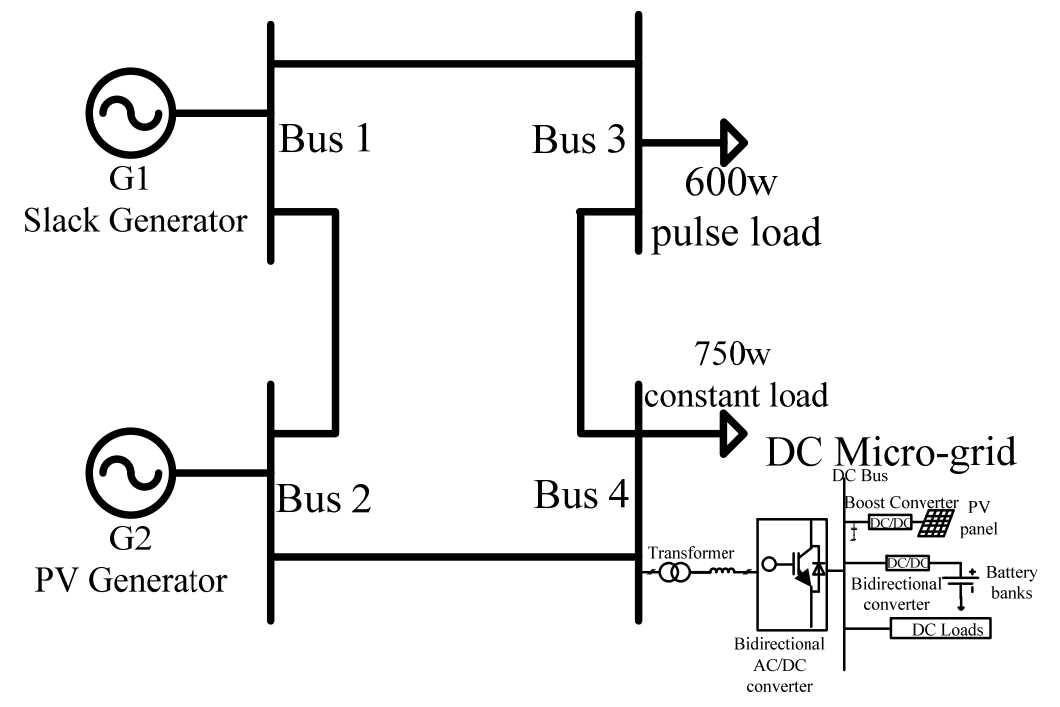

Figure 10.8 Hybrid AC-DC power system hardware platform configuration. 
to the AC subsystem. Also, the slack generator supplied $100 \mathrm{~W}$ to the system to control the system frequency. Generator G2 supplied a constant power of 550W to the hybrid power system.

During the four $600 \mathrm{~W}$ pulse load interference, the slack bus regulated the system frequency and increased the output power from $100 \mathrm{~W}$ to around $750 \mathrm{~W}$, and the output power from the DC micro grid and generator G2 were constant with very little variation.

After the four $600 \mathrm{~W}$ pulse loads, for some reason the slack generator G1 is disconnected from the rest of the system. The bidirectional AC-DC inverter immediately detects the system frequency drop which exceeds the bounds and lasts longer than the

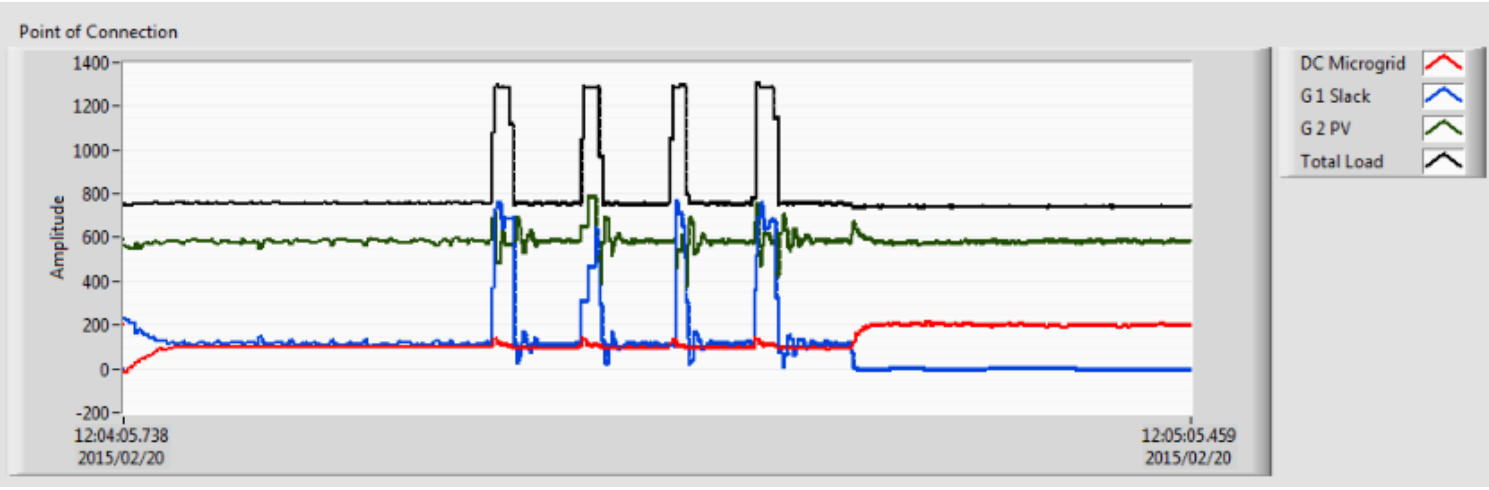

(a)

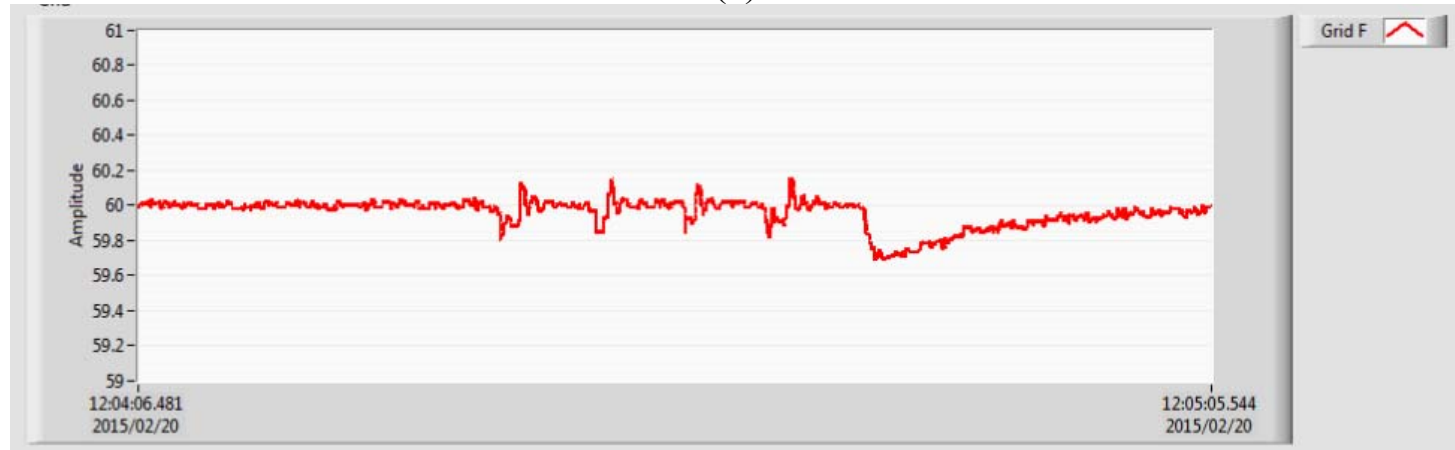

(b)

Figure 10.9 Hybrid AC-DC power system performance under pulse load and slack generator disconnection disturbances: (a) system power flow, (b) system frequency response. 
pulse load effect, therefore the frequency regulation function is enabled. After that, the DC micro grid injects $100 \mathrm{~W}$ more to compensate the loss of the slack generator, and the system frequency goes back to $60 \mathrm{~Hz}$ in a short time.

After the slack generator disconnection, four $300 \mathrm{~W}$ pulse loads happened in the system. Because generator G2 can only supply $550 \mathrm{~W}$, in order to keep the system stable the bidirectional AC-DC inverter should inject power from the DC subsystem to the AC subsystem. The load, output power from the two generators, and power from the DC micro grid is shown in figure 10.10 (a). The system frequency response is shown in figure 10.10 (b). From the results it can be seen that during the pulse load duration the

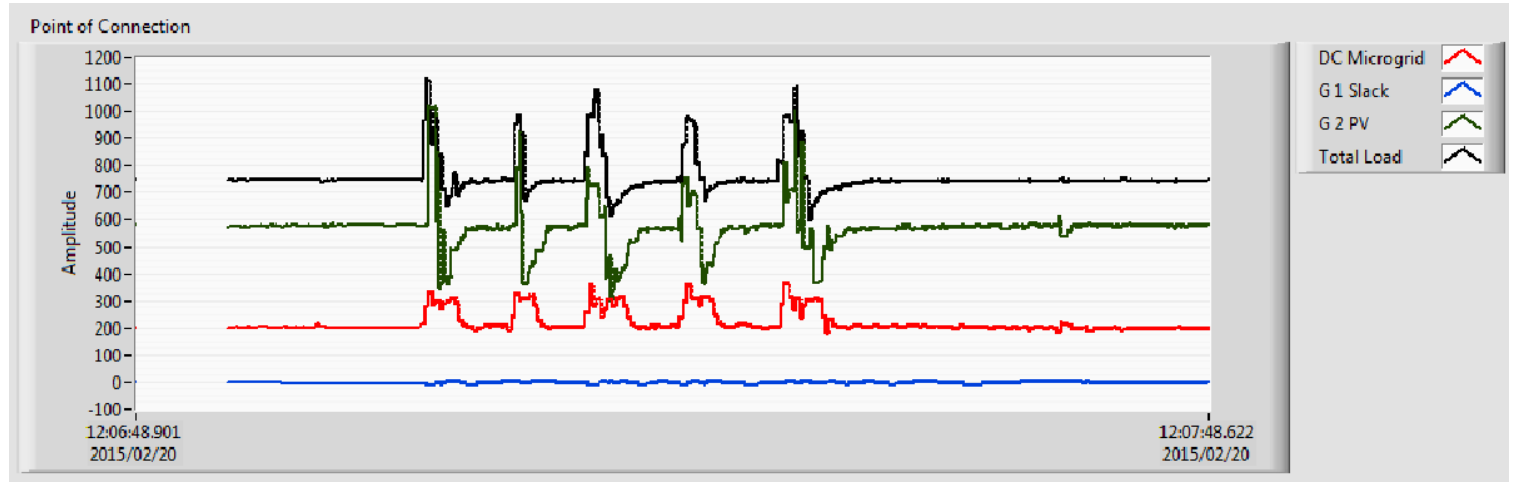

(a)

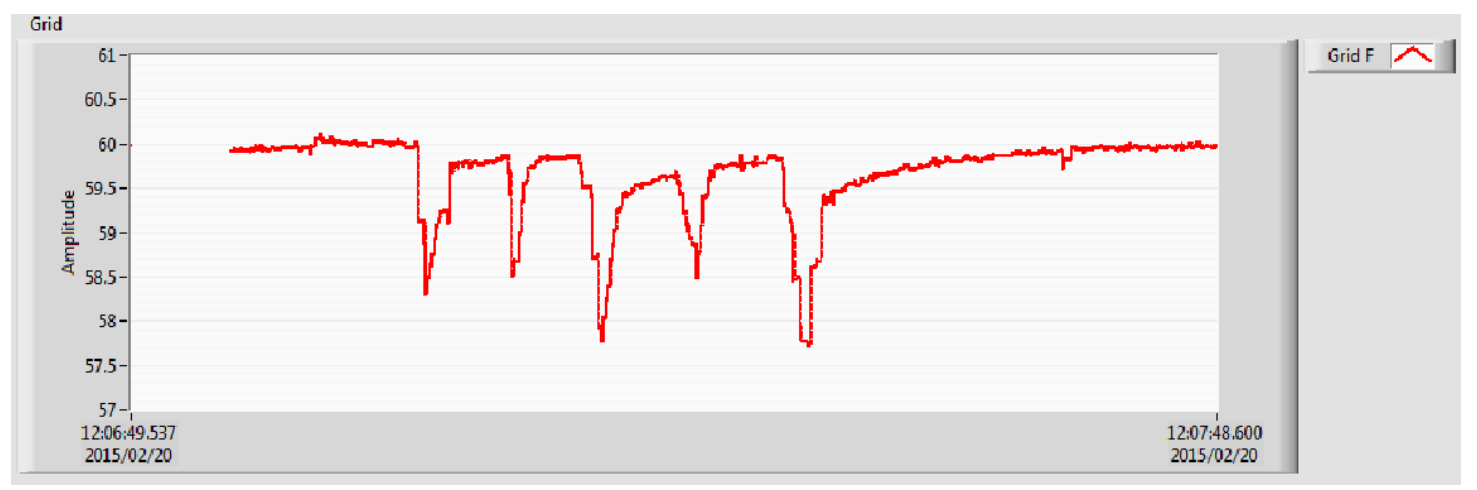

(b)

Figure 10.10 Hybrid AC-DC power system performance under pulse load with DC subsystem support: (a) system power flow, (b) system frequency response. 
DC micro grid can inject power to help the system regulate the frequency and the frequency only dropped to around $57.5 \mathrm{~Hz}$, which is close to the simulation results shown in Section 8.5.2.

For comparison, another experiment was done on the same setup without DC subsystem power support. The load, output power from the two generators, and power from the DC micro grid is shown in figure 10.11 (a). The system frequency response is shown in figure 10.11 (b). Since the DC subsystem doesn't support the AC subsystem in this case, the output power is always zero, and the power supplied by the slack generator G1 increased to $200 \mathrm{~W}$. During the first four $600 \mathrm{~W}$ pulse loads, the system is still stable since the slack generator G1 can regulate the system frequency. However, after the slack

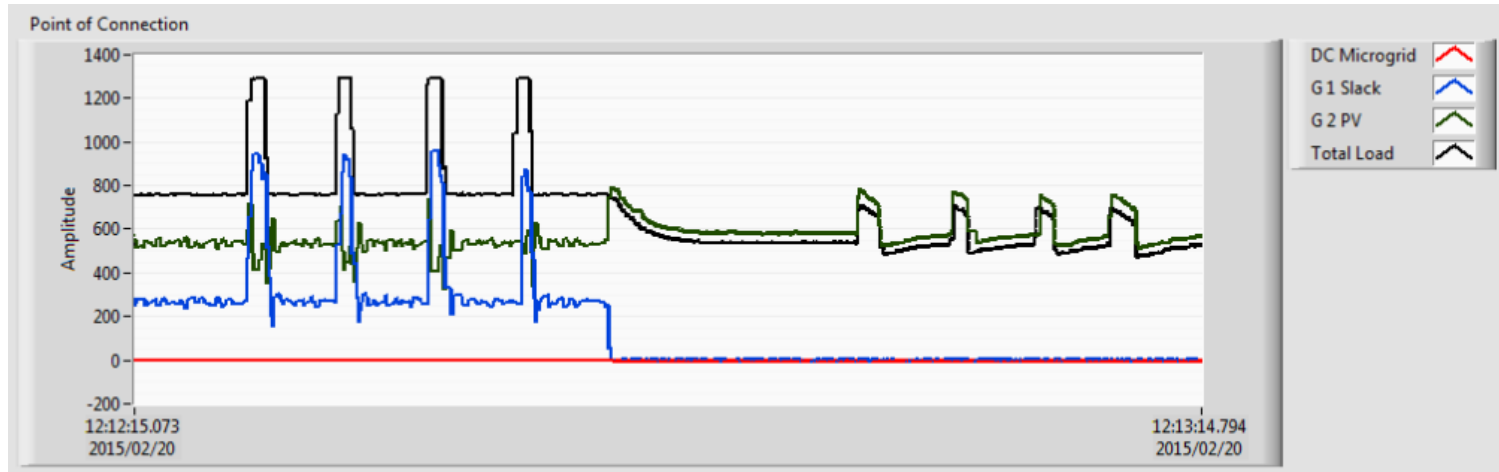

(a)

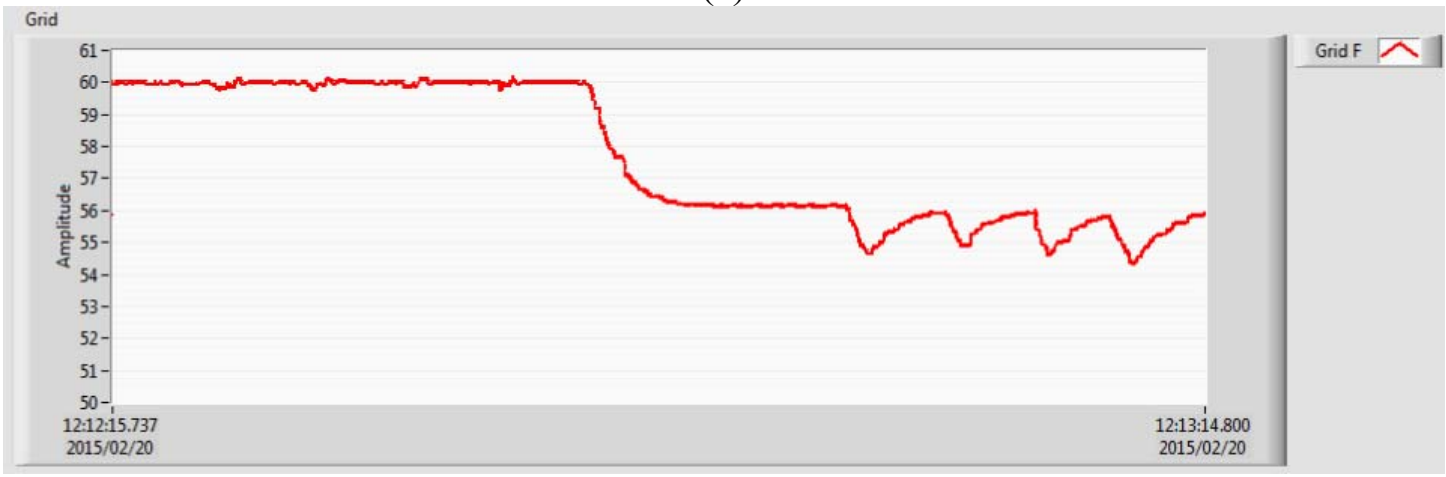

(b)

Figure 10.11 Hybrid AC-DC power system performance under pulse load without DC subsystem support: (a) system power flow, (b) system frequency response. 
generator disconnection, the system frequency drops to $56 \mathrm{~Hz}$ and stays at that value. Even worse, when the four $300 \mathrm{~W}$ pulse loads happen, the system frequency can drop to $54 \mathrm{~Hz}$. In this experiment, the rated output power of generator G2 is much larger than $750 \mathrm{~W}$. For protection reasons, the output power for generator G2 is not firmly limited to $750 \mathrm{~W}$, therefore during the four $300 \mathrm{~W}$ pulse load duration, generator G2's output power also increased, which relieved the system frequency drop.

\subsection{Conclusion}

In this chapter, the detailed description of a laboratory based PEV car park emulator is given. The PEV car park emulator is designed based on a hybrid AC-DC power system with both an AC micro grid and a DC micro grid including multiple components and power electronics devices. Five lithium-ion battery banks are used to emulate the charging behaviors of five PEVs. The proposed control methodology in Chapter 8 for PV emulator maximum power point tracking control, bidirectional DC-DC converter control, and bidirectional AC-DC inverter power decoupled control are applied to the hybrid power system. Meanwhile, the proposed battery banks remote control method in Chapter 9 is used in the PEV car park emulator. Hardware experiments are done with the results showing that the proposed PEV car park emulator can smartly charge the battery banks and help the AC micro grid regulate the system frequency through V2G services. 


\section{Conclusions and Future Work}

\subsection{Conclusions}

Hybrid AC-DC power systems that can be operated with multiple energy sources in both grid-connected and islanding modes are going to be one of the main architectures of future power systems. Hybrid AC-DC power systems integrated with renewable energy sources and PEVs will be an effective solution for reduction of whole system loss and greenhouse gas emissions. In this dissertation, different aspects related to the design, optimization, implementation, protection, and energy management of hybrid AC-DC power systems with PEVs and renewable energy sources are presented.

There are still challenges for future hybrid AC-DC power systems with a high penetration of renewable energy sources and plug-in electric vehicles (PEVs). Despite the challenges, with smart plan and control, renewable energy sources and PEV impacts can be greatly limited. In chapter 2, the introduction of hybrid AC-DC power system architecture is given. Together with this, the detailed operations of hybrid AC-DC power system's grid-connected and islanding modes are described with the challenges and system communication requirements. Also, the PEV charging impacts to the utility grid are analyzed. To limit those impacts, two possible solutions, V2G and V2V, are given with the description of present challenges and future markets. Chapter 3 presents the methods of harvesting solar energy from PV panels in hybrid power systems through DCDC boost converters and MPPT technology. In addition, the methodology of designing a library scaled PV emulator and DC-DC boost converter hardware is given. Also, the 
basic description of power conversion topologies with wind turbines is given with the information of how to maximize the output power from wind turbine power generation systems. Based on the above information, the renewable energy sources impact to the hybrid power system and how to overcome those challenges are described in detail.

An optimal sizing method for a renewable energy farm and energy storage devices in a hybrid power system is proposed in Chapter 4. Genetic algorithm is used to find the optimal solutions for both the renewable energy farm and energy storage devices. With the optimized scale of solar panels and wind turbine generators, the renewable energy farm can supply around twenty percent of the local load with less fluctuation and cost. The energy storage system with the optimized battery and ultra-capacitor sizes has the ability to smooth the energy flow from the renewable energy farm to the local load, which will greatly limit the impact on the utility grid from the renewable energy farm caused by uncertain factors such as the sudden change of solar irradiance and wind speed. A case study of the Key West, FL power system was performed.

To enhance the performance of the battery energy storage system, a fuzzy logic based battery bank management system with self-healing capabilities to protect itself from variations in the state of charge (SOC) and temperature of its battery banks is proposed in Chapter 5. The power consumed by the resistive elements in the equivalent circuit model used for each lithium-ion cell, along with the ambient temperature introduced into the system, allows for accurate estimation of the temperature. The proposed energy management system is able to effectively regulate both SOC and temperature during charging and discharging cycles for long periods of time and increase the safety of the whole system. 
To limit the PEVs charging impacts to the utility grid and utilize the PEVs for V2G services, a real-time energy management algorithm for PEVs charging in gridconnected smart charging car parks based on charging priority levels is developed and described in Chapter 6. The developed algorithm allows V2G and V2V functionalities and aims at minimizing the total cost of charging by handling the charging rates of the PEVs. A fuzzy agent was used as a component within the developed algorithm. Energy tariff, load demand and PV output power profiles are elements within the algorithm. The performance of the developed algorithm was tested by simulating its implementation on a car charging park connected to the IEEE standard 69-bus system at different penetration and distribution levels. The results show a reduction in the overall cost of charging as well as significant improvements in the voltage profile and the losses in the system. For feasibility concerns of the proposed PEV smart charging algorithm, the economic analysis of a real-time smart charging algorithm for 50,000 PEVs in a network with the consideration of $\mathrm{V} 2 \mathrm{G}$ frequency regulation service is given in Chapter 7. An economic model was built to estimate the daily and annual income of the proposed system with the consideration of battery degradation. The performance of the developed algorithm and the economic analysis of the whole model were tested by simulation. The economic analysis of the proposed system shows the capability of bringing considerable benefits to both the network and the owners of the PEVs.

Regarding the design and hardware implementation, a coordinated power flow control method for multiple power electronic devices is proposed in Chapter 8 for a laboratory based hybrid AC-DC power system operated in both grid-connected and islanding modes. The hybrid power system has a PV farm emulator and two local 
generators that supply energy to its DC and AC sides. Battery banks are connected to the DC bus through bidirectional DC-DC converters. The AC side and DC side are linked by the bidirectional AC-DC inverter. The system topology together with the control algorithms under both modes was tested with the influence of pulse loads and renewable energy farm output power variances.

To effectively control the battery energy storage system, the methodology of using transceivers and microcontrollers to remotely monitor and control the power flow of the energy storage system in a hybrid AC-DC power system is given in Chapter 9. The descriptions of how to utilize dSpace CP1004 and STM32F microcontrollers to control the power flow through bidirectional AC-DC inverters and DC-DC converters are given in detail. Also, using the STM32F to design a web server that acts as a central aggregator to monitor and control the power flow of five battery banks is presented. The proposed method gives a hardware solution to design the remote smart charging/discharging control for future PEV networks in a car park.

In the end, the detailed description of a laboratory based PEV car park emulator is given in Chapter 10. The PEV car park emulator is designed based on the hybrid AC-DC power system proposed in Chapter 8 and applied the control and energy management methodologies proposed in Chapter 4, 5, 6, 8, and 9. Five lithium-ion battery banks are used to emulate the charging behaviors of five PEVs. Hardware experiments were done with the results showing that the proposed PEV car park emulator can smartly charge the battery banks and help the AC micro grid regulate the system frequency through V2G services. 


\subsection{Future Work}

The PEV car park emulator hybrid power system introduced in this dissertation involved artificial intelligence, power electronics, communication, protection, and distributed control as key enabling technologies. The basic foundation and platform to research hybrid AC-DC power systems with the mitigation of impacts from PEVs, renewable energy sources, and critical loads was developed in this dissertation. Several optimization and smart operational schemes were proposed and verified in both simulation and hardware experiments. However, there are still many other ideas and technical aspects that need to be further researched to enhance the energy harvesting from renewable energy sources, limit the PEVs' charging impact to the utility grid, and improve the overall hybrid power system's reliability, stability, efficiency, and robustness.

To limit the PEVs' charging impact to the utility grid, a smart charging algorithm based on fuzzy logic is proposed in this dissertation. The central aggregator collects all the information in the hybrid power system and assigns charging rates for PEVs in different priority levels to smartly charge those PEVs. However, there is no communication between PEVs. Also, the PEVs can't negotiate with the central aggregator about the charging price. Therefore, some other intelligent control algorithm such as game theory needs to be applied to control the PEV network charging behaviors.

The design and operation of power electronic converters linking different components in the hybrid AC-DC power system were presented. The energy flows were controlled to keep the whole system stable by transferring power between the AC and DC subsystems and smart charging/discharging of the battery banks. However, the control of 
those bidirectional power electronic converters can be modified to perform more functions including system unbalancing compensation, harmonic filtering, self-healing, and system protection. Also, utilization of intelligent controllers instead of the conventional proportional integrative controllers may provide more flexibility to the power electronics devices. Novel power electronics device topologies need to be studied to limit the ripple-current and reduce the loss.

The developed PEV car park emulator hybrid AC-DC power system is operated in a distributed environment where communication plays an important role. A Zigbee wireless network was used in this dissertation as the communication protocol. However, since it is limited by the operational range, the Zigbee wireless transmitter can't be applied to control the charging of large PEV networks. Therefore, more investigations into the best communication architecture and its implementation are essential for coordination control of future large scale hybrid AC-DC power systems with huge numbers of PEVs. The future work on this topic should also discuss the communication type and protocols, the types of transferred messages, the needed storage databases, and the required devices. 


\section{LIST OF REFERENCES}

[1] R.G. Farmer and E.H. Allen, "Power System Dynamic Performance Advancement From History of North American Blackouts," in Proc. IEEE 2006 Power System conference and Exposition, Atlanta, Oct. 29-Nov. 1, 2006.

[2] H.A. Smolleck, S.J Ranade, N. R.Prasad, R.O. Velasco, "Effects of pulsed-power loads upon an electric power grid," Power Delivery, IEEE Transactions on , vol.6, no.4, pp.1629,1640, Oct 1991

[3] Kundur, Prabha. Power system stability and control. Edited by Neal J. Balu, and Mark G. Lauby. Vol. 7. New York: McGraw-hill, 19

[4] U.S. Energy Information Administration Annual Energy Ooulook 2014 with projiections to 2040http://www.eia.gov/forecasts/aeo/pdf/0383(2014).pdf

[5] American Council on Renewable Energy (ACORE) The Outlook For Renewable Energy In America 2014:

http://www.acore.org/files/pdfs/ACORE_Outlook_for_RE_2014.pdf

[6] Cox, Peter M., Richard A. Betts, Chris D. Jones, Steven A. Spall, and Ian J.

Totterdell. "Acceleration of global warming due to carbon-cycle feedbacks in a coupled climate model.” Nature 408, no. 6809 (2000): 184-187.

[7] Carrasco, Juan Manuel, et al. "Power-electronic systems for the grid integration of renewable energy sources: A survey." Industrial Electronics, IEEE Transactions on 53.4 (2006): 1002-1016.

[8] Eberle, Ulrich, and Rittmar von Helmolt. "Sustainable transportation based on electric vehicle concepts: a brief overview.” Energy \& Environmental Science 3.6 (2010): 689-699.

[9] U.S.A Presidential Memorandum-Federal Leadership on Energy Management: http://www.whitehouse.gov/the-press-office/2013/12/05/ presidentialmemorandum-federal-leadership-energy-management

[10] Halamay, D.A.; Brekken, T.K.A.; Simmons, A.; McArthur, S., "Reserve Requirement Impacts of Large-Scale Integration of Wind, Solar, and Ocean Wave Power Generation," Sustainable Energy, IEEE Transactions on , vol.2, no.3, pp.321,328, July 2011 
[11] Yilmaz, Murat, and Philip T. Krein. "Review of the impact of vehicle-to-grid technologies on distribution systems and utility interfaces." Power Electronics, IEEE Transactions on 28, no. 12 (2013): 5673-5689.

[12] Nguyen, H.K.; Song, J.B.; Han, Z., "Distributed Demand Side Management with Energy Storage in Smart Grid," Parallel and Distributed Systems, IEEE Transactions on, vol.PP, no.99, pp.1,1

[13] "Boeing 787 aircraft grounded after battery problem in Japan". BBC News. January 14, 2014. Retrieved January 16, 2014

[14] H. Rahimi-Eichi, U. Ojha, F. Baronti, M. Chow, "Battery Management System: An Overview of Its Application in the Smart Grid and Electric Vehicles," Industrial Electronics Magazine, IEEE , vol.7, no.2, pp.4,16, June 2013

[15] M. Yilmaz and P.T., Krein, "Review of the Impact of Vehicle-to-Grid Technologies on Distribution Systems and Utility Interfaces," Power Electronics, IEEE Transactions on , vol.28, no.12, pp.5673,5689, Dec. 2013

[16] U.S. Department of Energy, "Technical Challenges of Plug-in Hybrid Electric Vehicles and Imacts to the US Power System: Distribution System Analysis," Contract DE-1C05-76RL01830, January 2010

[17] Kramer, Bill, Sudipta Chakraborty, and Benjamin Kroposki. "A review of plug-in vehicles and vehicle-to-grid capability.” Industrial Electronics, 2008. IECON 2008. 34th Annual Conference of IEEE. IEEE, 2008.

[18] Xiaonan Lu; Guerrero, J.M.; Kai Sun; Vasquez, J.C.; Teodorescu, R.; Lipei Huang, "Hierarchical Control of Parallel AC-DC Converter Interfaces for Hybrid Microgrids," Smart Grid, IEEE Transactions on , vol.5, no.2, pp.683,692, March 2014.

[19] M. Hashmi, S. Hanninen, K. Maki, "Survey of smart grid concepts, architectures, and technological demonstrations worldwide," Innovative Smart Grid Technologies (ISGT Latin America), 2011 IEEE PES Conference on , vol., no., pp.1,7, 19-21 Oct. 2011

[20] X. Fang, S. Misra, G. Xue, D. Yang, "Smart Grid - The New and Improved Power Grid: A Survey," Communications Surveys \& Tutorials, IEEE, vol.14, no.4, pp.944,980, Fourth Quarter 2012

[21] Nejabatkhah, F.; Li, Y.W., "Overview of Power Management Strategies of Hybrid AC/DC Microgrid," Power Electronics, IEEE Transactions on , vol.PP, no.99, pp. 1,1 
[22] Kurohane, K.; Senjyu, T.; Yona, A.; Urasaki, N.; Goya, T.; Funabashi, T., “A Hybrid Smart AC/DC Power System," Smart Grid, IEEE Transactions on, vol.1, no.2, pp.199,204, Sept. 2010

[23] Salehi, V.; Mohamed, A.; Mazloomzadeh, A.; Mohammed, O.A., "Laboratory Based Smart Power System, Part I: Design and System Development," Smart Grid, IEEE Transactions on , vol.3, no.3, pp.1394,1404, Sept. 2012

[24] Salehi, V.; Mohamed, A.; Mazloomzadeh, A.; Mohammed, O.A., "Laboratory Based Smart Power System, Part II: Control, Monitoring, and Protection," Smart Grid, IEEE Transactions on, vol.3, no.3, pp.1405,1417, Sept. 2012

[25] Eghtedarpour, N.; Farjah, E., "Power Control and Management in a Hybrid AC/DC Microgrid, " Smart Grid, IEEE Transactions on , vol.5, no.3, pp.1494,1505, May 2014

[26] Shanthi, P.; Govindarajan, U.; Parvathyshankar, D., "Instantaneous power-based current control scheme for VAR compensation in hybrid AC/DC networks for smart grid applications," Power Electronics, IET , vol.7, no.5, pp.1216,1226, May 2014

[27] Fang, Xi; Misra, Satyajayant; Xue, Guoliang; Yang, Dejun, "Smart Grid — The New and Improved Power Grid: A Survey," Communications Surveys \& Tutorials, IEEE , vol.14, no.4, pp.944,980, Fourth Quarter 2012

[28] Nico Peterschmidt"Hybrid Power system-An Introduction”: http://www.wwindea.org/technology/ch05/en/5_3_1.html

[29] Kundur, Prabha. Power system stability and control. Eds. Neal J. Balu, and Mark G. Lauby. Vol. 7. New York: McGraw-hill, 1994.

[30] Balaguer, Irvin J., et al. "Control for grid-connected and intentional islanding operations of distributed power generation.” Industrial Electronics, IEEE Transactions on 58.1 (2011): 147-157.

[31] Feng XuePing; Liang Ying; Guo BingQing, "A new islanding method for distributed generation and its application in power system restoration," Advanced Power System Automation and Protection (APAP), 2011 International Conference on , vol.1, no., pp.378,383, 16-20 Oct. 2011

[32] Trujillo Rodriguez, C.; Velasco de la Fuente, D.; Garcera, G.; Figueres, E.; Guacaneme Moreno, J.A., "Reconfigurable Control Scheme for a PV Microinverter Working in Both Grid-Connected and Island Modes," Industrial Electronics, IEEE Transactions on , vol.60, no.4, pp.1582,1595, April 2013 
[33] Papaefthimiou, S.; Karamanou, E.; Papathanassiou, S.; Papadopoulos, M., "Operating policies for wind-pumped storage hybrid power stations in island grids, " Renewable Power Generation, IET , vol.3, no.3, pp.293,307, Sept. 2009

[34] Ma, Tan, and Osama Mohammed. "Economic analysis of real-time large scale PEVs network power flow control algorithm with the consideration of V2G services.” Industry Applications Society Annual Meeting, 2013 IEEE. IEEE, 2013.

[35] Renewable Energy, Wikipedia: http://en.wikipedia.org/wiki/Renewable_energy

[36] Johansson, Thomas B., and Laurie Burnham, eds. Renewable energy: sources for fuels and electricity. Island press, 1993.

[37] Carrasco, Juan Manuel, et al. "Power-electronic systems for the grid integration of renewable energy sources: A survey." Industrial Electronics, IEEE Transactions on 53.4 (2006): 1002-1016.

[38] Boyle, Godfrey. Renewable energy. OXFORD university press, 2004.

[39] Guilar, Nathaniel J., et al. "Integrated solar energy harvesting and storage." Very Large Scale Integration (VLSI) Systems, IEEE Transactions on 17.5 (2009): 627637.

[40] The World Wind Energy Association (2014). 2014 Half-year Report. WWEA. pp. $1-8$.

[41] Boulanger, Albert G., et al. "Vehicle electrification: Status and issues." Proceedings of the IEEE 99.6 (2011): 1116-1138.

[42] Anderson, David L. "An evaluation of current and future costs for lithium-ion batteries for use in electrified vehicle powertrains." Diss. Duke University, 2009.

[43] Yilmaz, Murat, and Philip T. Krein. "Review of battery charger topologies, charging power levels, and infrastructure for plug-in electric and hybrid vehicles." Power Electronics, IEEE Transactions on 28.5 (2013): 2151-2169.

[44] Yilmaz, Murat, and Philip T. Krein. "Review of the impact of vehicle-to-grid technologies on distribution systems and utility interfaces." Power Electronics, IEEE Transactions on 28.12 (2013): 5673-5689.

[45] Rao, S.; Berthold, F.; Pandurangavittal, K.; Blunier, B.; Bouquain, D.; Williamson, S.; Miraoui, A., "Plug-in Hybrid Electric Vehicle energy system using home-tovehicle and vehicle-to-home: Optimizaton of power converter operation," Transportation Electrification Conference and Expo (ITEC), 2013 IEEE , vol., no., pp.1,6, 16-19 June 2013 
[46] Long Zhao; Aravinthan, V., "Strategies of residential peak shaving with integration of demand response and V2H," Power and Energy Engineering Conference (APPEEC), 2013 IEEE PES Asia-Pacific , vol., no., pp.1,5, 8-11 Dec. 2013

[47] Mehdipoor, Hosssein; Jadid, Shahram, "Scheduling of Electrical Vehicles considering uncertainty of PV generations in Smart Homes," Electrical Power Distribution Networks (EPDC), 2014 19th Conference on , vol., no., pp.7,12, 6-7 May 2014

[48] Turker, H.; Hably, A.; Bacha, S., "Housing peak shaving algorithm (HPSA) with plug-in hybrid electric vehicles (PHEVs): Vehicle-to-Home (V2H) and Vehicle-toGrid (V2G) concepts," Power Engineering, Energy and Electrical Drives (POWERENG), 2013 Fourth International Conference on , vol., no., pp.753,759, 13-17 May 2013

[49] Dong, Q.; Niyato, D.; Wang, P.; Han, Z., "The PHEV Charging Scheduling and Power Supply Optimization for Charging Stations," Vehicular Technology, IEEE Transactions on, vol.PP, no.99, pp.1,1

[50] Fox, G.H., "Electric Vehicle Charging Stations: Are We Prepared?” Industry Applications Magazine, IEEE, vol.19, no.4, pp.32, 38, July-Aug. 2013

[51] Xu, Z.; Su, W.; Hu, Z.; Song, Y.; Zhang, H., "A Hierarchical Framework for Coordinated Charging of Plug-In Electric Vehicles in China," Smart Grid, IEEE Transactions on, vol.PP, no.99, pp.1,1

[52] Bai, X.; Qiao, W., "Robust Optimization for Bidirectional Dispatch Coordination of Large-Scale V2G," Smart Grid, IEEE Transactions on, vol.PP, no.99, pp.1,1

[53] Ota, Y.; Taniguchi, H.; Nakajima, T.; Liyanage, K.M.; Baba, J.; Yokoyama, A., "Autonomous Distributed V2G (Vehicle-to-Grid) Satisfying Scheduled Charging," Smart Grid, IEEE Transactions on , vol.3, no.1, pp.559,564, March 2012

[54] Ma, Z.; Zou, S.; Liu, X., “A Distributed Charging Coordination for Large-Scale Plug-In Electric Vehicles Considering Battery Degradation Cost," Control Systems Technology, IEEE Transactions on, vol.PP, no.99, pp.1,1

[55] Wencong Su; Mo-Yuen Chow, "Performance Evaluation of an EDA-Based LargeScale Plug-In Hybrid Electric Vehicle Charging Algorithm," Smart Grid, IEEE Transactions on, vol.3, no.1, pp.308,315, March 2012

[56] Jon Wellinhoff (2009). David B. Sandalow, ed. Chapter 4: The CashBack Car (1st ed.). The Brookings Institution. pp. 65-82. ISBN 978-0-8157-0305-1. in "Plug-in Electric Vehicles: What Role for Washington?" 
[57] Spotnitz, R.; Franklin, J. (2003). "Abuse behavior of high-power, lithium-ion cells”. Journal of Power Sources 113: 81. doi:10.1016/S0378-7753(02)00488-3

[59] Ma, Tan, and Osama A. Mohammed. "Optimal Charging of Plug-in Electric Vehicles for a Car-Park Infrastructure.” Industry Applications, IEEE Transactions on 50.4 (2014): 2323-2330.

[60] Spotnitz, R.; Franklin, J. (2003). "Abuse behavior of high-power, lithium-ion cells". Journal of Power Sources 113: 81. doi:10.1016/S0378-7753(02)00488-3

[61] Lu, Languang, Xuebing Han, Jianqiu Li, Jianfeng Hua, and Minggao Ouyang. "A review on the key issues for lithium-ion battery management in electric vehicles." Journal of power sources 226 (2013): 272-288

[62] Artifical Nueral Network, Wikipedia: http://en.wikipedia.org/wiki/Artificial_neural_network

[63] Genetic Algorithms, Wikipedia: http://en.wikipedia.org/wiki/Genetic_algorithm

[64] Fuzzy Logic, Wikipedia: http://en.wikipedia.org/wiki/Fuzzy_logic

[65] Ang, Kiam Heong, Gregory Chong, and Yun Li. "PID control system analysis, design, and technology." Control Systems Technology, IEEE Transactions on 13.4 (2005): 559-576.

[66] Gao, Jingcheng, et al. "A survey of communication/networking in smart grids." Future Generation Computer Systems 28.2 (2012): 391-404.

[67] U.K. Madawala and D.J. Thrimawithana, "A Bidirectional Inductive Power Interface for Electric Vehicles in V2G Systems," IEEE Trans. on Industrial Electronics, vol. 58, no. 10, pp. 4789-4896, Oct. 2011.

[68] C. Quinn, D. Zimmerle, and T.H. Bradley, "The Effect of Communication Architecture on the Availability, Reliability, and Economics of Plug-in Hybrid Electric Vehicle-to-Grid Ancillary Services," Journal of Power Sources, vol. 195, no. 5, pp. 1500-1509, March 2010.

[69] M.K. Lee, R.E. Newman, H.A. Latchman, S. Katar, and L. Yonge, "HomePlug 1.0 Powerline Communication LANs - Protocol Description and Performance Results," International Journal of Communication Systems, vol. 16, pp. 447-473, 2003.

[70] P. Kulshrestha, K. Swaminathan, M.-Y. Chow, and S. M. Lukic, "Evaluation of ZigBee Communication Platform for Controlling the Charging of PHEVs at a 
Municipal Parking Deck," in Proc. 2009 IEEE Vehicle Power and Propulsion Conference, pp. 1211-1214, Dearborn, MI, Sep. 7-10, 2009.

[71] T. Markel, M. Kuss, and P. Denholm, "Communication and Control of Electric Drive Vehicles Supporting Renewables," in Proc. 2009 Vehicle Power and PropulsionConference, pp. 27-34, 2009.

[72] R. Liu, L. Dow and E. Liu, "A Survey of PEV Impacts on Electric Utilities" Innovative Smart Grid Technologies (ISGT), 2011IEEE PES, pp. 1-8, 2011.

[73] M. Kintner Meyer, K. Schneider and R. Pratt, "Impacts Assessment of PHEV on Electric Utilities and Regional U.S. Power Grids, Part 1: Technical Analysis," PNNL SA-53700, November, 2007.

[74] P. Denholm and W. Short, "An Evaluation of Utility System Impacts and Benefits of Optimally Dispatched Plug-In Hybrid Electric Vehicles," Technical Report, NREL/TP-620-40293, July 2006

[75] EPRI, "Environmental Assessment of Plug-In Hybrid Electric Vehicles," Technical Report 1015325, July 2007.

[76] F. Locment, M. Sechilariu and C. Forgez, "Electric Vehicle Charging System with PV Grid-Connected Configuration," Vehicle Power and Propulsion Conference (VPPC) 2010 IEEE, pp. 1-6, Sept. 2010.

[77] A. Bedir, B. Ozpineci and J. E. Christian, "The Impact of Plug-In Hybrid Electric Vehicle Interaction with Energy Storage and Solar Panels on the Grid for a Zero Energy House," Transmission and Distribution Conference and Exposition 2010 IEEE PES, pp. 1-6, April 2010.

[78] C. Hutson, G.K. Venayagamoorthy and K.A. Corzine, "Intelligent Scheduling of Hybrid and Electric Vehicle Storage Capacity in a Parking Lot for Profit Maximization in Grid Power Transactions," Energy 2030 Conference 2008. IEEE, pp. 1-8, Nov. 2008.

[79] A. Krkoleva, V. Borozan, A. Dimeas and N. Hatziargyriou, "Gossip based Message Dissemination Schemes in Future Power Systems," Intelligent System Application to Power Systems (ISAP) 2011 16th International Conference on, pp. 1-6, Sept. 2011.

[80] P. Mitra, G.K. Venayagamoorthy, "Intelligent Coordinated Control of a Wind Farm and Distributed Smartparks," Industry Applications Society Annual Meeting (IAS) 2010 IEEE, pp. 3-7, Oct. 2010. 
[81] Mathew, B.; Varghese, J., "Electric vehicle as STATCOM and real power flow controller for wind energy conversion system," Emerging Research Areas: Magnetics, Machines and Drives (AICERA/iCMMD), 2014 Annual International Conference on , vol., no., pp.1,4, 24-26 July 2014

[82] G. G.K. Venayagamoorthy and P.P. Mitra, "Smartpark Shock Absorbers for Wind Farms," IEEE Transactions on Energy Conversion, vol. 26, no. 3, pp. 990-992, Sept. 2011.

[83] Yuting Mou; Hao Xing; Zhiyun Lin; Minyue Fu, "Decentralized Optimal Demand-Side Management for PHEV Charging in a Smart Grid," Smart Grid, IEEE Transactions on , vol.6, no.2, pp.726,736, March 2015.

[84] S. Deilami, A.S. Masoum, P.S. Moses and M.A.S. Masoum, "Real-Time Coordination of Plug-In Electric Vehicle Charging in Smart Grids to Minimize Power Losses and Improve Voltage Profile," IEEE Transactions on Smart Grid, vol. 2, no. 3, pp. 456-467, September 2011.

[85] M. Wakihara, and Y. Osamu, eds. "Lithium ion batteries," John Wiley \& Sons, 2008.

[86] P. E. Ross,“Boeing's Battery Blues,” IEEE Spectrum, 1-2. February 2013

[87] M. Uno and K. Tanaka, "Accelerated ageing testing and cycle life prediction of supercapacitors for alternative battery applications,"in Proc. 2011, 33rd Int. Telecommunications Energy Conf. (INTELEC 2011), Piscataway, NJ, p. 6.

[88] T. Huria, M. Ceraolo, J. Gazzarri, R. Jackey "High fidelity electrical model with thermal dependence for characterization and simulation of high power lithium battery cells," In Electric Vehicle Conference (IEVC), 2012 IEEE International (pp. 1-8). IEEE. March, 2012

[89] T. Kim, W. Qiao, and L. Qu, "A multicell battery system design for electric and plug-in hybrid electric vehicles," Electric Vehicle Conference (IEVC),. IEEE. March, 2012.

[90] X. Lu; K. Sun, J.M. Guerrero, J.C. Vasquez, L. Huang, "State-of-Charge Balance Using Adaptive Droop Control for Distributed Energy Storage Systems in DC Microgrid Applications, ” Industrial Electronics, IEEE Transactions on, vol.61, no.6, pp.2804,2815, June 2014

[91] War of Currents, Wikipedia, http://en.wikipedia.org/wiki/War_of_Currents

[92] U.S. Department of Energy, "Communications Requirements of Smart Grid Techniques," October 5, 2010. 
[93] J. D. Dogger, B. Roossien, and F. D. J. Nieuwenhout, "Characterization of Li-Ion Batteries for Intelligent Management of Distributed Grid-Connected Storage," IEEE Trans. Energy Conversion, vol. 26, no. 1, pp. 256-263, March 2011.

[94] S. L. Andersson, A.K. Elofsson, M.D. Galus, L. Göransson, S. Karlsson, and F. Johnsson, "Plug-in Hybrid Electric Vehicles as Regulating Power Providers: Case studies of Sweden and Germany," Energy Policy, vol. 38, no. 6, pp. 2751-2762, 2010.

[95] Zpryme, "Smart Grid Insights: V2G,” 2010.

[96] Wikipedia, Renewable Energy Systems: http://en.wikipedia.org/wiki/Renewable_Energy_Systems

[97] Paiva, J.E.M.S.; Carvalho, A.S., "An integrated hybrid power system based on renewable energy sources with electric wind MPPT," IECON 2010 - 36th Annual Conference on IEEE Industrial Electronics Society, vol., no., pp.2981,2987, 7-10 Nov. 2010

[98] Wikipedia, Solar Energy, http://en.wikipedia.org/wiki/Solar_energy

[99] Natural Resources Defense Council, Renew Energy in Florida State: http://www.nrdc.org/energy/renewables/florida.asp

[100] U.S Department of Energy, Florida Wind Resource Map and Wind potential Capacity:

http://apps2.eere.energy.gov/wind/windexchange/wind_resource_maps.asp?stateab $=\mathrm{fl}$

[101] S.K. Kollimalla, M.K. Mishra, "A Novel Adaptive P\&O MPPT Algorithm Considering Sudden Changes in the Irradiance," Energy Conversion, IEEE Transactions on, vol.29, no.3, pp.602,610, Sept. 2014

[102] K. Sundareswaran, S. Peddapati, S. Palani, "Application of random search method for maximum power point tracking in partially shaded photovoltaic systems," Renewable Power Generation, IET, vol.8, no.6, pp.670,678, August 2014

[103] P. Sharma, V. Agarwal, "Exact Maximum Power Point Tracking of GridConnected Partially Shaded PV Source Using Current Compensation Concept," Power Electronics, IEEE Transactions on, vol.29, no.9, pp.4684,4692, Sept. 2014

[104] U.S. Energy Information Administration, "Annual Energy Review 2011" http://www.eia.gov/forecasts/aeo/pdf/0383(2014).pdf 
[105] Tan Ma; Mohammed, O., "Plug-in vehicles car park photovoltatic farm construction for cost and emission reductions," Energy Conversion Congress and Exposition (ECCE), 2013 IEEE , vol., no., pp.5179,5184, 15-19 Sept. 2013.

[106] U.S.A Presidential Memorandum-Federal Leadership on Energy Management: http://www.whitehouse.gov/the-press-office/2013/12/05/ presidentialmemorandum-federal-leadership-energy-management

[107] Chi Su; Weihao Hu; Zhe Chen; Yanting Hu, "Mitigation of power system oscillation caused by wind power fluctuation," Renewable Power Generation, IET , vol.7, no.6, pp.639,651, Nov. 2013

[108] Tan Ma; Serrano, B.; Mohammed, O., "Fuzzy logic based power and thermal management system design for multi-cell lithium-ion battery bank protection and operation," Power Systems Conference (PSC), 2014 Clemson University , vol., no., pp.1,5, 11-14 March 2014

[109] Chen, S.X.; Gooi, H.B.; Wang, M. Q., "Sizing of Energy Storage for Microgrids," Smart Grid, IEEE Transactions on , vol.3, no.1, pp.142,151, March 2012

[110] Lin Xu; Xinbo Ruan; Chengxiong Mao; Buhan Zhang; Yi Luo, "An Improved Optimal Sizing Method for Wind-Solar-Battery Hybrid Power System," Sustainable Energy, IEEE Transactions on , vol.4, no.3, pp.774,785, July 2013

[111] L. Cristaldi, M. Faifer, M. Rossi, and F. Ponci, “A Simple Photovoltaic Panel Model: Characterization Procedure and Evaluation of the Role of Environmental Measurements," IEEE Transactions on Instrumentation and Measurement, vol. 61, no. 10, pp. 2632-2641, Oct. 2012

[112] G. M. Masters, "Photovoltaic Materials and Electrical Characteristics" in Renewable and Efficient Electric Power Systems. Hoboken, NJ: John Wiley \& Sons, 2004, ch. 8., sec. 8.6, pp. 497-499.

[113] A. Mohamed, V. Salehi, O. Mohammed, "Real-Time Energy Management Algorithm for Mitigation of Pulse Loads in Hybrid Microgrids", IEEE Transactions on Smart Grid, Dec. 2012, vol. 3, no.4, pp 1911-1922

[114] X. Liu; P. Wang; P. C. Loh, "A Hybrid AC/DC Microgrid and Its Coordination Control," IEEE Transactions on Smart Grid, vol.2, no.2, pp.278,286, June 2011doi: 10.1109/TSG.2011.2116162

[115] Kim, T., Qiao, W., \& Qu, L “A multicell battery system design for electric and plug-in hybrid electric vehicles," Electric Vehicle Conference (IEVC),. IEEE. March, 2012 
[116] Lawder, M.T.; Suthar, B.; Northrop, P.W.C.; De, S.; Hoff, C.M.; Leitermann, O.; Crow, M.L.; Santhanagopalan, S.; Subramanian, V.R., "Battery Energy Storage System (BESS) and Battery Management System (BMS) for Grid-Scale Applications," Proceedings of the IEEE , vol.102, no.6, pp.1014,1030, June 2014

[117] M. Uno and K. Tanaka, "Accelerated ageing testing and cycle life prediction of supercapacitors for alternative battery applications,"in Proc. 2011, 33rd Int. Telecommunications Energy Conf. (INTELEC 2011), Piscataway, NJ, p. 6.

[118] Battery University, Charging lithium-ion battery: http://batteryuniversity.com/learn/article/charging_lithium_ion_batteries

[119] The European Association for Advanced Rechargeable Batteries, "Safety of lithium-ion batteries", June 2013

[120] T. Huria, M. Ceraolo, J. Gazzarri, R. Jackey "High fidelity electrical model with thermal dependence for characterization and simulation of high power lithium battery cells," In Electric Vehicle Conference (IEVC), 2012 IEEE International (pp. 1-8). IEEE. March, 2012

[121] Nutkani, I.U.; Poh Chiang Loh; Peng Wang; Blaabjerg, F., "Cost-Prioritized Droop Schemes for Autonomous AC Microgrids," Power Electronics, IEEE Transactions on , vol.30, no.2, pp.1109,1119, Feb. 2015

[122] Rocabert, J.; Luna, A.; Blaabjerg, F.; Rodríguez, P., "Control of Power Converters in AC Microgrids," Power Electronics, IEEE Transactions on, vol.27, no.11, pp.4734,4749, Nov. 2012

[123] Coelho, E.A.A.; Cortizo, P.C.; Garcia, P.F.D., "Small-signal stability for parallelconnected inverters in stand-alone AC supply systems," Industry Applications, IEEE Transactions on , vol.38, no.2, pp.533,542, Mar/Apr 2002

[124] Jaehong Kim; Guerrero, J.M.; Rodriguez, P.; Teodorescu, R.; Kwanghee Nam, "Mode Adaptive Droop Control With Virtual Output Impedances for an InverterBased Flexible AC Microgrid," Power Electronics, IEEE Transactions on , vol.26, no.3, pp.689,701, March 2011

[125] Huang, P.-H.; Liu, P.-C.; Xiao, W.; El Moursi, M.S., "A Novel Droop-Based Average Voltage Sharing Control Strategy for DC Microgrids," Smart Grid, IEEE Transactions on, vol.PP, no.99, pp.1,1

[126] Augustine, S.; Mishra, M.K.; Lakshminarasamma, N., "Adaptive Droop Control Strategy for Load Sharing and Circulating Current Minimization in Low-Voltage Standalone DC Microgrid," Sustainable Energy, IEEE Transactions on , vol.6, no.1, pp.132,141, Jan. 2015 
[127] Xiaonan Lu; Guerrero, J.M.; Kai Sun; Vasquez, J.C., “An Improved Droop Control Method for DC Microgrids Based on Low Bandwidth Communication With DC Bus Voltage Restoration and Enhanced Current Sharing Accuracy,” Power Electronics, IEEE Transactions on , vol.29, no.4, pp.1800,1812, April 2014

[128] Xiaonan Lu; Kai Sun; Guerrero, J.M.; Vasquez, J.C.; Lipei Huang, "State-ofCharge Balance Using Adaptive Droop Control for Distributed Energy Storage Systems in DC Microgrid Applications," Industrial Electronics, IEEE Transactions on , vol.61, no.6, pp.2804,2815, June 2014

[129] Berggren, B.; Linden, K.; Majumder, R., "DC Grid Control Through the Pilot Voltage Droop Concept-Methodology for Establishing Droop Constants," Power Systems, IEEE Transactions on , 2014 vol.PP, no.99, pp.1,9

[130] J. Voelcker, “How Green Is My Plug-In?” Spectrum, IEEE, vol.46, no.3, pp.42-58, March 2009.

[131] "Technology roadmap: Electric and plug-in hybrid electric vehicles (EV/PHEV)," International Energy Agency (IEA), 2011.

[132] A.Y. Saber, G.K. Venayagamoorthy, "Plug-in Vehicles and Renewable Energy Sources for Cost and Emission Reductions," IEEE Transactions on Industrial Electronics, vol.58, no.4, pp.1229-1238, April 2011

[133] J. Tomicand W. Kempton, "Using fleets of electric drive vehicles for grid support,” J. Power Sources, vol. 168, no. 2, pp. 459-468, Jun. 2007.

[134] S.G. Wirasingha, N. Schofield, A. Emadi, "Plug-in hybrid electric vehicle developments in the US: Trends, barriers, and economic feasibility," Vehicle Power and Propulsion Conference, 2008. VPPC '08. IEEE, vol., no. pp.1-8, 3-5 Sept. 2008.

[135] S. Deilami, A.S. Masoum, P.S. Moses and M.A.S. Masoum, "Real-Time Coordination of Plug-In Electric Vehicle Charging in Smart Grids to Minimize Power Losses and Improve Voltage Profile," IEEE Transactions on Smart Grid, vol. 2, no. 3, pp. 456-467, September 2011.

[136] A.S. Masoum, S. Deilami, P.S. Moses, M.A.S. Masoum and A. Abu-Siada, "Smart load management of plug-in electric vehicles in distribution and residential networks with charging stations for peak shaving and loss minimisation considering voltage regulation," Generation, Transmission \& Distribution, IET, vol.5, no.8, pp. 877-888, August 2011. 
[137] P. Mitra, G.K. Venayagamoorthy and K.A. Corzine, "Smartpark as a Virtual STATCOM," IEEE Transactions on Smart Grid, vol. 2, no. 3, pp. 445-455, September 2011

[138] Yifeng He; Venkatesh, B.; Ling Guan, "Optimal Scheduling for Charging and Discharging of Electric Vehicles," Smart Grid, IEEE Transactions on , vol.3, no.3, pp.1095,1105, Sept. 2012

[139] A. Mohamed, M. Elshaer and O. Mohammed, "Bi-Directional AC-DC/DC-AC converter for Power Sharing of Hybrid AC/DC Systems," in Proc. of IEEE PES General Meeting 2011, Detroit, Michigan, USA.

[140] M. Elshaer, A. Mohamed and O. Mohammed, "Integration of Sustainable Energy Sources into DC Zonal Electric Distribution Systems," in Proc. of IEEE PES General Meeting 2011, Detroit, Michigan, USA.

[141] A. Mohamed, M. Elshaer, O. Mohammed, "High-Quality Integration of Fuel Cells Energy into Electric Grids," in Proc. Of 4th International Symposium on Resilient Control Systems, ISRCS 2011, Boise, Idaho, USA, Aug. 9-11, 2011.

[142] A. Mohamed and O. Mohammed, "Smart Optimal Control of DC-DC Boost Converter in PV Systems," in Proc. of the Transmission and Distribution Conference and Exposition: Latin America (T\&D-LA), 2010 IEEE/PES, pp. 403410, Sao Paulo, Brazil, Nov. 2010.

[143] E. Weisstein, "Making Math World," Mathematical Journal 10, 2007

[144] L. Cam, Lucien, "The central limit theorem around 1935," Statistical Science, pp. 78-91, 1986.

[145] C. Liu, K.T. Chau, D. Wu, and S. Gao, "Opportunities and challenges of vehicleto-home, vehicle-to-vehicle and vehicle-to-grid technologies," Proceeding of the IEEE, Invited Paper, vol. 101, no. 11, pp. 2409-2427, Nov. 2013.

[146] S. Shahidinejad, S. Filizadeh, E. Bibeau, "Profile of Charging Load on the Grid Due to Plug-in Vehicles,"IEEE Transactions on Smart Grid, vol.3, no.1, pp.135,141, March 2012.

[147] H. Sekyung, H. Soohee and K. Sezaki, "Economic assessment on V2G frequency regulation regarding the battery degradation," Innovative Smart Grid Technologies (ISGT), 2012 IEEE PES, vol., no., pp.1-6, 16-20 Jan. 2012.

[148] H. Sekyung, H. Soohee and K. Sezaki, "Estimation of Achievable Power Capacity from Plug-in Electric Vehicles for V2G Frequency Regulation: Case Studies for 
Market Participation,” Smart Grid, IEEE Transactions on, vol.2, no.4, pp.632-641, Dec. 2011.

[149] L. Sanna, "Driving the solution the plug-in hybrid electric vehicle", EPRI Journal, pp. 8-17, Fall 2005

[150] General Electric 1.5sle Datasheet. [Online]. Available: http://www.gepower.com/prod_serv/products/wind_turbines/en/downloads/ge_15_ brochure.pdf

[151] C. K. Sao and P. W. Lehn, "Control and power management of converterfed MicroGrids," IEEE Trans. Power Syst., vol. 23, no. 3, pp. 1088-1098, Aug. 2008.

[152] A. Mohamed, F. Carlos, T. Ma, M. Farhadi, O. Mohammed, "Operation and protection of photovoltaic systems in hybrid AC/DC smart grids," IECON 2012 38th Annual Conference on IEEE Industrial Electronics Society, pp.1104-1109, 25-28 Oct. 2012.

[153] R. H. Lasseter and P. Paigi, "Microgrid: A conceptual solution," in Proc. IEEE 35th PESC, Jun. 2004, vol. 6, pp. 4285-4290.

[155] A. Mohamed, V. Salehi and O. Mohammed, "Reactive Power Compensation in Hybrid AC/DC Networks for Smart Grid Applications," in Proc. Innovative Smart Grid Technologies Conf., ISGT Europe 2012, Berlin, Germany, October 14-17, 2012

[156] M. Schuerger, H. Johal, L. Roose, M. Matsuura,and R. Piwko, "Catching Some Rays: Variable Generation Integration on the Island of Oahu," Power and Energy Magazine, IEEE, vol.11, no.6, pp.33,44, Nov. 2013

[157] M. Ahlstrom, et al "Knowledge Is Power: Efficiently Integrating Wind Energy and Wind Forecasts," Power and Energy Magazine, IEEE, vol.11, no.6, pp.45,52, Nov. 2013

[158] N. Miller, C.Loutan, Shao Miaolei, K. Clark, "Emergency Response: U.S. System Frequency with High Wind Penetration," Power and Energy Magazine, IEEE, vol.11, no.6, pp.63,71, Nov. 2013

[159] Paul C. Krause, Oleg Wasynczuk, and Scott D. Sudhoff, "Analysis of electric machinery and drive systems" Purdue University, second edition, IEEE Press, Wiley-Interscience, 2002.

[160] T. Ma, A. Elsayed and O. A. Mohammed, "Educational Experiments in Renewable Energy Analysis, Forecasting, and Management in Hybrid Power System," 
Published in the Proceedings of the 120th ASEE Annual Conference \& Exposition, Atlanta, USA, 23-26 June 2013

[161] S. Bu, F.R.Yu, Y. Cai, X.P. Liu, "When the Smart Grid Meets Energy-Efficient Communications: Green Wireless Cellular Networks Powered by the Smart Grid," Wireless Communications, IEEE Transactions on , vol.11, no.8, pp.3014,3024, August 2012

[162] Teranishi, T. (n.d.). Tera Term Help Index. Retrieved September 10, 2013. http://ttssh2.sourceforge.jp/manual/en/

[163] Martin Hebel, G. B. Getting Started with XBEE RF Modules. Retrieved November 12, 2013. http://www.makershed.com/v/vspfiles/assets/images/122-32450xbeetutorial-v1.0.1.pdf

[164] Waijung Blockset: http://waijung.aimagin.com/

[165] Monti, A.; Santi, E.; Dougal, R.A.; Riva, M., "Rapid prototyping of digital controls for power electronics," Power Electronics, IEEE Transactions on , vol.18, no.3, pp.915,923, May 2003 
VITA

TAN MA

1985

Born, Xinyang, China

2007

B.E., Automation, Huazhong University of Science and Technology, Wuhan, China

2009

M.S., Control Science and Control Engineering, Huazhong University of Science and Technology, Wuhan, China

2009-2011

Graduate Teaching Assistant, Florida International University

$2011-2012$

Research Assistant, Florida International University

2013

Dissertation Evidence Acquisition Fellowship, Florida International University

$2014-2015$

Dissertation Year Fellowship, Florida International University

2015

Best Research Assistant of ECE Department, Florida International University

\section{PUBLICATIONS AND PRESENTATIONS}

1. Tan Ma, O. A. Mohammed, "Optimal Charging of Plug-in Electric Vehicles for a Car Park Infrastructure," IEEE Transactions on Industry Applications, vol.50, no.4, pp.2323-2330, July-Aug. 2014.

2. Tan Ma, O. A. Mohammed, "Economic Analysis of Real-time Large Scale PEVs Network Power Flow Control Algorithm with the Consideration of V2G Services," IEEE Transactions on Industry Applications, vol. 50, no.6, pp.4272-4280, Nov.-Dec. 2014.

3. A. Mohammed, V. Salehi, Tan Ma and O. A. Mohammed, "Real-Time Management Algorithm for Plug-in Hybrid Electric Vehicle Charging Parks Involving Sustainable Energy," IEEE Transactions on Sustainable Energy, vol. 5, no. 2, pp. 577-586, April 2014. 
4. Mehmet H. C, Tan Ma, O.A. Mohammed, "Distributed Protection and Dynamic Reconfiguration of Microgrids Based on Enhanced Selective Interlocking," IEEE Transactions on Smart Grid(under review)

5. L. Guo, H. Deng, B. Himed, Tan Ma and Z. Geng, "Waveform Optimization for Transmit Beamforming with MIMO Antenna," IEEE Transactions on Antenna and Propagation, vol. 63, No. 2, pp.543-552, Feb. 2015.

6. Tan Ma, O. A. Mohammed, "Plug-in Vehicles Car Park Photovoltaic Farm Construction for Cost and Emission Reductions," IEEE Energy Conversion Congress and Exposition (ECCE) 2013, pp. 5179-5184, 15-19 Sept. 2013.

7. Tan Ma, B. Serrano, O. A. Mohammed, "Fuzzy Logic Based Power and Thermal Management System Design for Multi-cell Lithium-ion Battery Bank Protection and Operation," IEEE Power System Conference (PSC) 2014, pp. 1-5, Clemson University, March 11-14, 2014.

8. Tan Ma, B. Serrano, O. A. Mohammed, "Distributed Control of Hybrid ACDC Microgrid with Solar Energy, Energy Storage and Critical Load," IEEE Power System Conference (PSC) 2014, Clemson University, March 11-14, 2014.

9. Tan Ma, B. Serrano, O. A. Mohammed, "Educational Approach to the Methodology of Implementing Wireless Control of Power Flow in Hybrid Power Systems," American Society for Engineering Education (ASEE) annual meeting 2014, Indianapolis, IN, June 15-18, 2014.

10. Tan Ma, O. A. Mohammed, "Economic Analysis of Real-time Large Scale PEVs Network Power Flow Control Algorithm with the Consideration of V2G Services," IEEE Industry Applications Society Annual Meeting 2013, pp.1-8, Oct. 2013.

11. Tan Ma, O. A. Mohammed, "Real-Time Plug-In Electric Vehicles Charging Control for V2G Frequency Regulation," IEEE IECON 2013-39th Annual Conference of the IEEE Industrial Electronics Society, Vienna, Austria.

12. Tan Ma; A. Mohamed; O. A. Mohammed, "Optimal charging of plug-in electric vehicles for a car park infrastructure," IEEE Industry Applications Society Annual Meeting (IAS) 2012, pp.1,8, 7-11 Oct. 2012

13. Mehmet H. C, Tan Ma, O.A. Mohammed, "Aggregated Active Distribution Networks for Secondary Control of Islanded Power Systems", Accepted by 2015 IEEE PES General Meeting

14. Tan Ma, C. R. Lashway, Y. Song, Osama Mohammed, "Optimal Renewable Energy Farm and Energy Storage Sizing Method for Future Hybrid Power System”, ICEMS 2014. 